\title{
TRANSLATION OF (OSTEO)CHONDRAL REGENERATIVE STRATEGIES
}

FROM NATURE'S DESIGN TO THE EQUINE MODEL

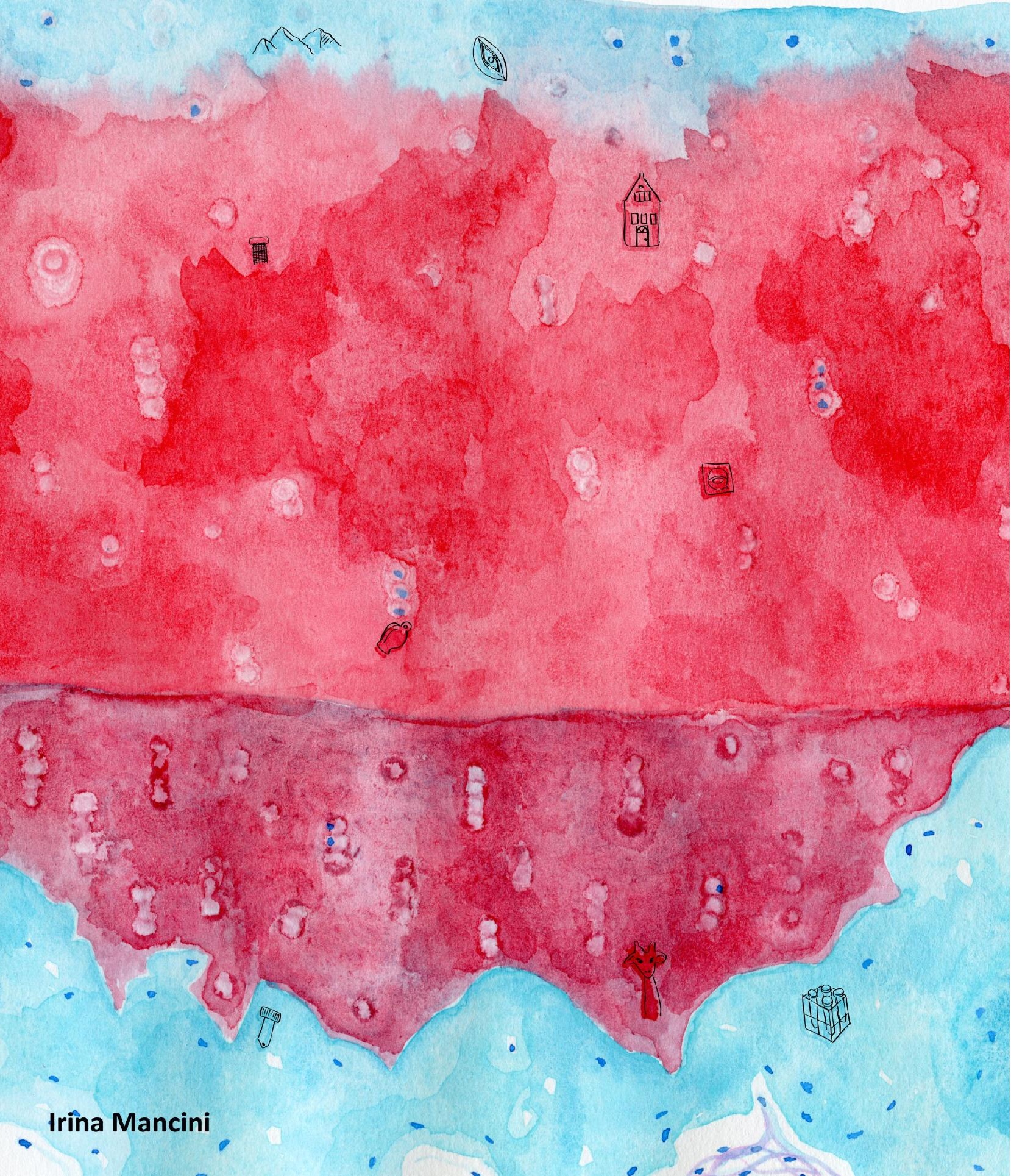





\section{Translation of (osteo)chondral regenerative strategies: \\ from nature's design to the equine model}

Irina Mancini 
Translation of (osteo)chondral regenerative strategies:

from nature's design to the equine model

Irina A.D. Mancini

$\mathrm{PhD}$ thesis, Faculty of Veterinary Medicine, Utrecht University, the Netherlands

ISBN: 978-94-6361-493-1

Cover design and artwork: Irina Mancini

The centaur depicted before chapter 8 is based on the anatomical table of the Codex Extincta Animalia by S.E.Black, M.D. as created by E.B. Hudspeth.

Layout: Fred Regoli

Printed by: Optima Grafische Communicatie, Rotterdam, the Netherlands

Copyright @ Irina Mancini 2020.

All rights reserved. No part of this thesis may be reproduced, distributed, stored in a retrieval system of any nature or transmitted in any form or by any means, without prior written consent of the author. The copyright of the articles that have been published has been transferred to the respective journals.

Printing of this thesis was financially supported by the Department of Clinical Sciences, Faculty of Veterinary Medicine, Utrecht University. 


\section{Translation of (osteo)chondral regenerative strategies}

From nature's design to the equine model

Translatie van (osteo)chondrale regeneratieve strategieën

Van het natuurlijk ontwerp tot het paardenmodel

(met een samenvatting in het Nederlands)

Traslazione di strategie rigenerative (osteo)condrali

Dal disegno della natura al modello equino

(con un riassunto in italiano)

Translation de stratégies de régénération (osteo)chondrale Du dessein de la nature au modèle équin

(avec un résumé en français)

\section{Proefschrift}

ter verkrijging van de graad van doctor aan de Universiteit Utrecht op gezag van de rector magnificus, prof.dr. H.R.B.M. Kummeling ingevolge het besluit van het college voor promoties in het openbaar te verdedigen op dinsdag 8 december 2020 des middags te 12.45 uur door

Irina Anna Diletta Mancini

geboren op 15 maart 1985

te Roma, Italië 


\section{Promotoren:}

Prof. dr. P.R. van Weeren

Prof. dr. J. Malda

\section{Copromotor:}

Dr. R. Levato 
Al mio omino pasticcino 



\title{
TABLE OF CONTENTS
}

\author{
Introduction
}

Chapter 1 General introduction, outline and research questions

Part I Understanding the native osteochondral template

Chapter 2 Effects of body mass on microstructural features of the osteochondral unit:

a comparative analysis of 37 mammalian species

Chapter 3 Nature's design of the osteochondral unit: microstructural differences between terrestrial and aquatic mammals

Part II Translation of hydrogel-based regenerative strategies in the equine model for cartilage repair

Chapter 4 Lessons from a long-term equine study on cartilage repair with a cocolture of chondrons and MSCs in gelatin methacryloyl hydrogel

Chapter 5 Fixation of hydrogel constructs for cartilage repair in the equine model: a challenging issue

Chapter 6 A composite hydrogel-3D printed thermoplast osteochondral anchor as an example for a zonal approach to cartilage repair: in vivo performance in a long-term equine model

Chapter 7 Comment on "A composite hydrogel-3D printed thermoplast osteochondral anchor as an example for a zonal approach to cartilage repair"

\section{Discussion and summary}

Chapter 8 General discussion

Nederlandse samenvatting $\quad 202$

Riassunto in italiano $\quad 206$

Résumé en français $\quad 212$

Acknowledgements $\quad 218$

$\begin{array}{ll}\text { List of publications } & 220\end{array}$

Professional biography $\quad 222$ 


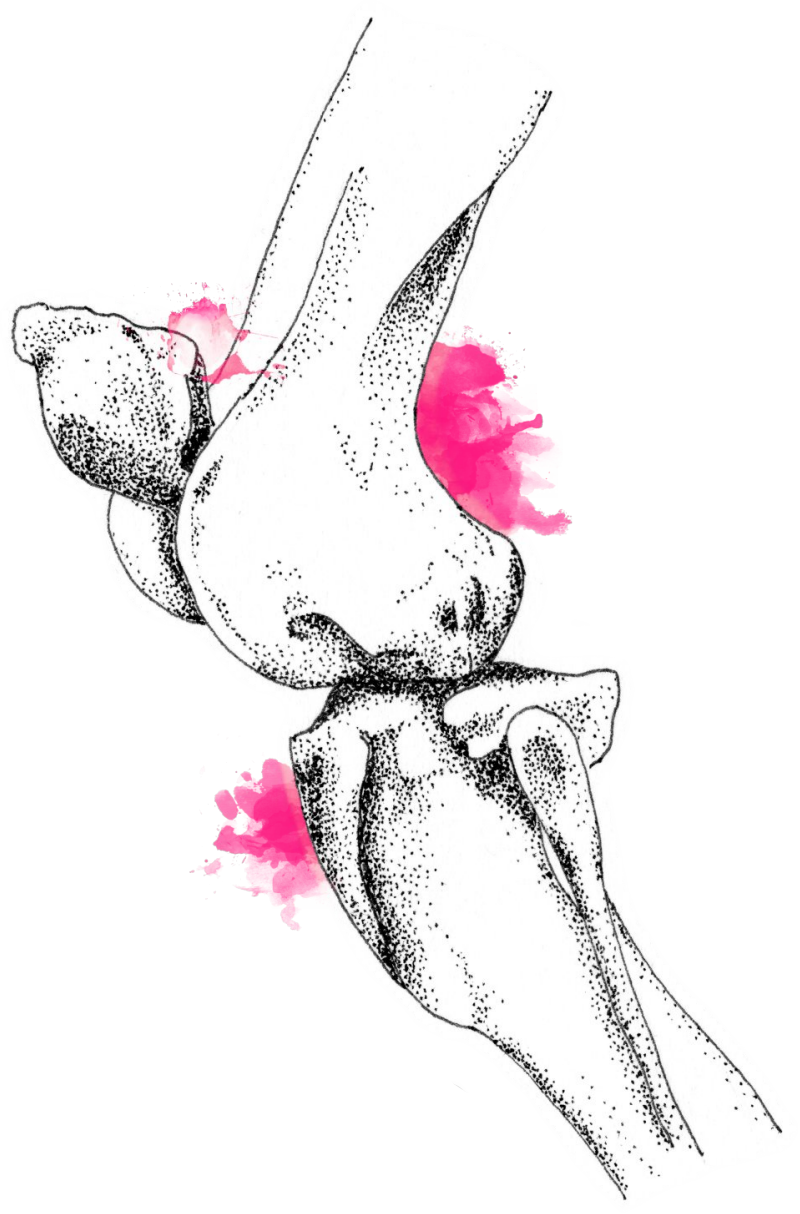




\section{Chapter}

General introduction, outline and research questions 


\section{INTRODUCTION}

\section{Forever on the move: Nature's template of the mammalian joint}

In nature, mammals represent an extraordinary successful group, with an incredible diversity of species, morphologies, physiologies and behaviours, that has collectively succeeded in colonizing all continents and living even in the most inhospitable environments on the planet [1]. A good example of their extreme variation in morphology is the vast range in size and body mass. Mammals can weigh from $2 \mathrm{~g}$ (bumble-bee bat) to over 150,000 kg (blue whale) [2]. The adaptivity of mammals is exemplified by the variations of limb extremities, where the original five fingers have undergone a number of modifications. They have become wings for flying in bats, and, even within closely related groups like Cetartiodactyla (cows, pigs, whales and porpoises), they have evolved into attributes as widely different as hooves and flippers [3]. These morphological adaptations have enabled different species to live on land, in trees or in water, ultimately exhibiting different solutions to the same problem: locomotion. Indeed, moving in different environments poses different physical demands. Water, for example, is denser than air [4], and while on land gravity is the principal force, in water other forces, like buoyancy and drag, become more important [5]. Mammals have adapted to their environments [6] and the joints, as the literally pivotal elements of the extremities, have followed these adaptations.

In all mammals, the function of diarthrodial joints is to allow articulation between the bony components of the skeleton with minimal friction and to transmit and dampen the forces generated by locomotion [7]. For effective function, the different components of the joint have to work in synergy, so articular cartilage, the subchondral bone plate and the underlying (subchondral) trabecular bone, i.e. the osteochondral unit, can be considered as a single functional entity $[8,9]$. Each component is constituted by a tissue that is highly specialized to carry out specific functions. Articular cartilage has developed structural and biochemical characteristics enabling it to sustain intermittent loading, while allowing lubrication of the surface; the underlying bone has adapted to carry and disperse loads and to provide support and anchor points for other structures needed for locomotion. 
Cartilage is an avascular, aneural, heterogeneous tissue, composed of a extracellular matrix (ECM) rich in water (70\%) trapped by an interconnected network of collagen type II and proteoglycans [7]. In this matrix, a small percentage of cells $(5-10 \%)$ called chondrocytes reside [10], linked to the matrix through proteins that allow them to sense and respond to mechanical forces [11]. Based on chondrocyte morphology and tissue composition, hyaline cartilage can be divided into three different zones: the superficial, middle and deep zone. Beneath these zones there is a layer of calcified cartilage that forms the interface with the subchondral bone (Fig. 1). Each portion is specialized to resist the load it experiences and ensure the optimal biomechanical response required by its location [12]. The total mechanical strength of the tissue is a result of the interaction between the water trapped within the matrix and the sum of the different bonds between the tissue components. Collagen type II fibres, in particular, are linked with covalent bonds [13], and form an organized matrix with arch-like structures; these fibres, run parallel to the articulating surface in the superficial layer and become perpendicular to the surface in the deep layer (Fig. 1).

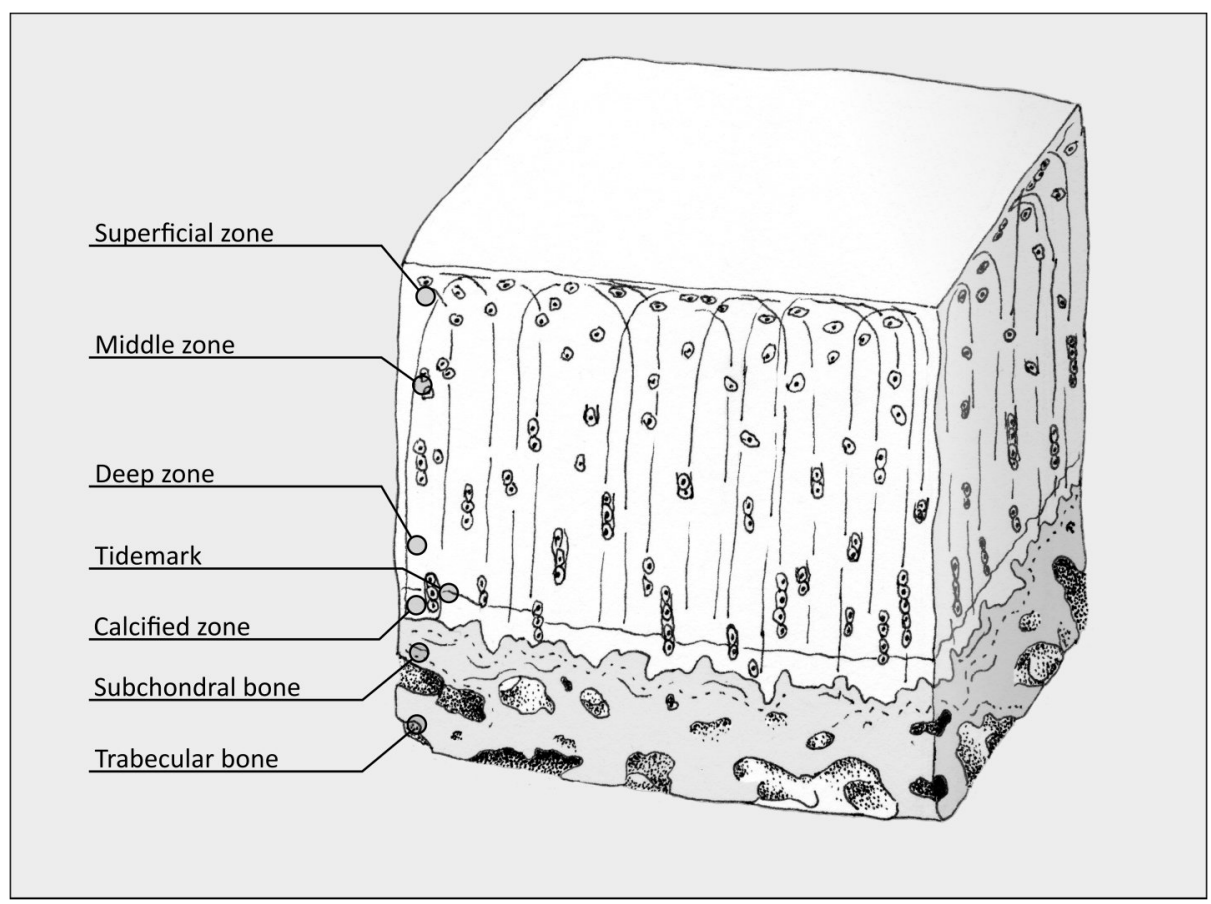

Figure 1. Structure of the osteochondral unit. 
Aggrecan is the main proteoglycan present in the tissue, and includes glycosaminoglycans, such as keratan sulphate and chondroitin sulphate, that branch from the aggrecan core protein. The aggrecan macromolecules are connected to long hyaluronic acid polysaccharide chains, and the repeating sulphate groups confer the network a high net negative charge. This, in turn, attracts cations within the tissue, therefore providing high osmotic tissue pressure, which acts to resist compression during loading [12]. This combination of networks results in a resilient, yet elastic tissue that can withstand both shear and compressive forces, such as the ones applied to cartilage during the load-bearing moments of locomotion. When loading of cartilage occurs, a pressure gradient and volumetric change is created within the tissue, causing the interstitial fluid to pass through the porous solid portion of the cartilage matrix out of the tissue, into the synovial joint space [13]. The fluid moving through the solid phase generates a high frictional resistance, which is the primary mechanism responsible for the viscoelastic behaviour of articular cartilage during compression [13]. Subsequently, upon unloading, fluid is drawn back into the cartilage, bringing in new nutrients from the synovial space [14]. It is, therefore, necessary for cartilage to be subjected to a regular loading routine in order to effectuate the exchange of nutrients and waste products, just as circulation would in other tissues [14].

Cartilage is separated from the underlying bone by a tidemark and a layer of calcified cartilage. The calcified cartilage features mineralization in the form of crystals of various calcium salts, and the tidemark represents the boundary between the calcified and the non-calcified (hyaline) parts of articular cartilage. At the tidemark, part of the collagen network continues into the calcified layer, with both perpendicular and randomly oriented fibres. Around these fibres, there is increased calcified material, and a band of flattened fibrils running parallel to the undulating surface of calcified cartilage [15]. This organization suggests that the tidemark provides a tethering mechanism for the collagen fibrils in the deepest portion of articular cartilage, preventing shear forces from detaching the fibres [15]. The calcified cartilage layer is also hypothesized to act as a mechanical coupling and buffer between cartilage and bone [16]. The interface, however, is not continuous, and in limited portions of the interface, there is direct contact between the hyaline cartilage and the underlying bone [17]. 
Underneath the calcified cartilage is the subchondral bone plate, a cortical portion of bone between the trabecular bone and the calcified zone [18]. It is constituted by dense cancellous bone, which is fenestrated by numerous spaces that gradually become elongated perpendicularly to the articular surface, forming the trabecular bone [19]. Biochemically, bone is a biphasic material, composed by a large percentage (60\%) of inorganic components, primarily calcium phosphates, such as hydroxyapatite crystals, a smaller percentage (30\%) of organic compounds, such as collagen type I, proteoglycans and glycosaminoglycans, and water (10\%) [20]. The combination of these components confers the tissue strength while allowing a certain amount of elasticity [21]. The composition of subchondral bone is thought to be designed especially to disperse axial loads across the joint, alleviating the burden on the articular cartilage above [22].

Bone and articular cartilage have developed very different ways to respond to biomechanical loading. One of the main differences resides in the bone's ability to continuously adapt its response to mechanical loading and therefore remain functional throughout the lifetime of an individual [23, 24]. This is also true for subchondral bone, as illustrated by studies on its maturation in various species, such as cows and horses $[25,26]$.

Subchondral bone displays an adaptive response to both acute stresses and prolonged chronic changes of applied forces, enabled by the productive and resorptive actions of osteoblasts and osteoclasts [27]. The rich vascularization and innervation also facilitate the response within the bone both to physiologic and pathologic changes [27]. This is in sharp contrast to the situation in the articular cartilage, where the collagen network is only able to adapt during maturation in juveniles in a process defined as functional adaptation $[28,29]$. However, in mature adults there is no collagen turn-over [30], which severely limits the tissue's capacity to change and heal.

The trabecular bone situated directly underneath the subchondral bone has a similar biochemical composition as the subchondral bone, but displays a more hierarchical organization. It is composed of lightweight internal lattices called trabeculae that provide structural support, particularly in the portions closer to the joint. These structures ensure resilience and strength of the bone tissue while maintaining an efficient volume/strength ratio [24, 31]. Trabecular bone shares with the subchondral plate the high adaptability, already described in the $19^{\text {th }}$ century, in the quest of a model to explain trabecular 
bone architecture [32] and bone adaptation to loading [33]. In the 1860s the collaboration between anatomist Von Meyer and civil engineer Culmann sparked the first hypothesis of the existence of a match between acting forces and bone architecture. This concept evolved with the key contribution of Julius Wolff into the law of bone microarchitectural adaptation in response to mechanical loads, refined by the biologist Wilhelm Roux who intuited the role in the adaptation process of biophysical self-regulation mechanisms at cellular level [34]. Wolff's description of the relationship between bone remodelling and loading, was expanded at the end of the 20th century by Frost with the mechanostat hypothesis [35]. Wolff's law stated that bone adapts to loading by decreasing bone mass when loaded below a certain threshold and by increasing it in reaction to loading above this threshold, achieving an optimal balance between bone weight and strength [32]. Frost later observed that trabeculae were driven to align along the main direction of loading, because the trabeculae with a different orientation would not reach the threshold loading and, therefore, eventually disappear, overall increasing the anisotropy degree of trabecular bone [36].

\section{The regenerative approach to joint disease}

\section{Regenerative medicine}

Regenerative medicine is a relatively new branch of medicine that aspires to assist the body in restoring normal function of damaged tissues, by replacing, engineering or regenerating tissues and organs. To achieve its goals, regenerative medicine combines biomaterials, cells and regenerative cues [37, 38]. The continuous advancement in stem cell research contributes increasing knowledge to the regenerative medicine field on possible cell sources, as well as on techniques for cell differentiation and instruction [39]. The principles of life sciences and engineering convene in regenerative medicine, where they are applied toward the development of biological substitutes that restore, maintain or improve the function of a tissue [40]. The advances in development of biomaterials and bioengineering techniques have opened a virtually indefinite number of possibilities for the production of implants and scaffolds with different biological and mechanical characteristics to match a variety of tissues [41]. 
In regenerative medicine multiple disciplines converge, where specialists of science and medicine collaborate to revolutionize treatment, offering a possible cure rather than the treatment of symptoms [38].

Joint disease - when the template fails

Unlike the majority of tissues, articular cartilage is avascular, with low cell density. This particular structure, while crucial for function, also implies limited access to nutrients and circulating repair cells. Moreover, this also results in an environment with limited availability of nutrients and oxygen (from $10 \%$ at the surface to $<1 \%$ in the deep layer) [42]. The combination of cytoplasmic isolation of the cells, low cell density, and absence of cell division under physiological conditions contributes to the low regenerative potential of articular cartilage [42]. Another factor in the limited intrinsic repair capacity of cartilage, is represented by the almost inexistent turnover of the collagen fibres: the collagen matrix appears to be essentially permanent, with no capability of replacement even in pathologic conditions [30]. Therefore, when damage occurs, cartilage tissue lacks the potential to naturally produce functional new tissue, which eventually leads to impaired joint function [43, 44].

The natural healing process of cartilage is characterized by the production of a predominantly fibrous repair tissue, initially produced in sufficient quantity to fill the gap created by the damage, and partially integrated with the surrounding healthy tissue. However, because of its inferior biomechanical properties compared to native cartilage, in the long term the repair tissue cannot withstand the repeated loading caused by weight-bearing and locomotion and cannot function properly, leading ultimately to its degeneration and failure [45].

When native cartilage is damaged, small focal defects are created. These defects can affect the tissue thickness only partially, or in its entirety. Untreated, partial thickness defects tend to heal poorly [46]. These focal defects are characterized by a local, confined loss of tissue and function in a healthy joint and, therefore, can be temporarily asymptomatic [47]. Lesions of this type are often trauma-induced and mostly prevalent in younger patients with an active lifestyle, which, particularly in the initial absence of symptoms, can lead over time to the expansion of the damage which becomes generalized, eventually causing joint inflammation, swelling, pain, and, 
therefore, a decrease in mobility [48].

Osteochondral lesions involve cartilage and the underlying bone, and can be caused by acute or chronic trauma, as well as by genetic factors [49-51]. When the damage reaches the subchondral bone, this triggers an inflammatory reaction in the tissue, with migration of cells to the damaged area and production of chemotactic signals for a repair response that eventually produces a fibrous tissue, rich in collagen type I, again less performant than native cartilage. In osteochondral defects, both bone and cartilage are injured, so degeneration may happen within a shorter time range than in chondral defects, resulting into earlier development of osteoarthritis [52].

Osteoarthritis is an inflammatory disease that involves the whole joint, from cartilage to bone, synovium and ligaments: the inflammation triggered by the degeneration can involve one or potentially all these tissues, furthering the vicious cycle of degeneration and inflammation, and bringing pain that ultimately limits mobility [48]. Osteoarthritis is a degenerative, chronic disease that affects most commonly knees, hands, spine, shoulder and hip joints. Its prevalence is associated to ageing, but also to a variety of risk factors which include lack of exercise, obesity, genetic predisposition, occupational injuries and gender [53]. Osteoarthritis represents one of the most common causes of disability in older adults, and is estimated to affect $10-15 \%$ of all adults aged over 60 [54], with a prevalence in Europe varying from $2.8 \%$ in Romania to $18.3 \%$ in Hungary [55].

Early regenerative therapies for chondral and osteochondral defects

Treatment of focal defects traditionally involves debridement of the damaged cartilage and microfracture of the subchondral plate, which allows cells from the bone marrow to invade the defect and stimulates a repair response [56]. However, because the repair tissue produced is inferior to native tissue, as pointed out above, there is still a clinical need for improvement of therapies, and regenerative cell-based strategies have, therefore, been investigated as a possible alternative. The first generation of cartilage cell-based repair was the 2-step autologous chondrocyte implantation $(\mathrm{ACl})$, where chondrocytes were placed in the defect and covered with a periosteal flap; the periosteal flap was substituted in later years by a synthetic collagen sheet [57]. In the third generation version of this therapy, a porous collagen matrix in which the cells are cultured prior to implantation 
( $\mathrm{MACl})$ is used, eliminating the need for further fixation, and allowing the intervention to be performed arthroscopically [58].

When damage reaches the bone, creating osteochondral lesions, larger areas of tissue are affected, and traditional debridement and microfracture is likely to yield insufficient repair [59]. Therefore, more complex therapies are required, such as the combination of implantation of autologous bone and autologous expanded chondrocytes, which requires two separate surgical interventions [60,61]. A common alternative is mosaicplasty, which is a surgical technique that consists in the transfer of autologous osteochondral plugs with viable bone and cartilage from non-weight-bearing areas of the joint into the defects $[62,63]$. A great advantage of this strategy is that it requires a single surgical intervention, however tissue harvest causes damage and may lead to donor-site morbidity, being additionally associated to poor filling of tissue in the areas between the grafts [62]. Therefore, the idea of producing a scaffold analogue to an osteochondral graft by means of tissue engineering is appealing, and has led researchers to venture into this area.

\section{Osteochondral tissue engineering}

The engineering of a regenerative osteochondral construct is a complex process in which several choices need to be made. These include the choice of (1) the biomaterial(s), (2) whether cells will be used and if so, the type(s) of those cells and (3) of the optimal manufacturing technique.

\section{Engineering cartilage and bone}

In load-bearing tissues, understanding the interrelationship between composition, architecture and function is crucial for determining the criteria for the design of a potentially functional construct. In the case of cartilage, the native tissue is able to provide compressive and tribological properties that allow successful withstanding of a joint's biomechanical challenges [64]. Bone regeneration research has resulted in several clinically successful products [65], based on a variety of suitable materials for bone scaffolds, which include combinations of collagen, tricalcium phosphate and hydroxyapatite [66]. In bone defects, regenerative strategies often aim at providing temporary support, while allowing sufficient mechanical stimulation for the new bone production. In cartilage defects, no solution exists yet that is capable of performing the same task, and the continuous loading on newly formed, less 
performant fibrocartilage, leads ultimately to failure of the repair tissue [64]. Some regenerative strategies for cartilage, like allograft transplantation, have yielded positive results [67], possibly because the collagen structure -the most difficult feature of the cartilage tissue to reproduce- was contained in the transplanted material. But no technique yet can accurately replicate the architecture of the collagen network, which represents a virtually immutable element of the cartilaginous tissue [68].

\section{- Biomaterials}

Biomaterials for (osteo)chondral regeneration, such as hydrogels, can be applied by simple casting for repair of chondral defects, where hydrogels act as a cell carrier, or can be used for osteochondral defect repair as part of more complex, multiphasic scaffold-based therapies with constructs composed by different materials and cell types. Several hydrogel-based materials have been investigated as potential candidates for cartilage repair [69], including products based on gelatine [70], collagen [71], proteoglycans [71] and poly(ethylene glycol) [72]. Natural polymers typically have lower manufacturing costs, they are biodegradable and can be constituted by extracellular matrix analogues, such as collagen, and thus better mimic the native environment of the target tissue. These polymers, however, show biological variation between batches, display lower chemical, mechanical and thermal stability, and involve a risk of immuno-rejection and disease transmission [72]. Synthetic polymers offer a highly tailorable and repeatable structure and can be characterized in detail with respect to their physical, chemical and mechanical properties. Synthetic polymers can also be designed to minimize risks of toxicity and immunogenicity and pose virtually no risk of disease transmission. However, they generally need biological functionalization to resemble the target tissue's environment [72].

While hydrogels are great candidates for tissue engineering, several requirements need to be met for successful use in regenerative medicine, including appropriate cross-linking to guarantee stability of the polymers, adequate biomaterial degradability and biocompatibility and support for cell survival and proliferation [69]. The lack of biomaterials that are versatile enough to satisfy all these prerequisites, has been an important limiting factor in the progress of the field $[73,74]$. Given the significant biomechanical forces on implants for (osteo)chondral applications, assurance of adequate 
mechanical properties in particular represents a key challenge $[75,76]$.

\section{- Cell sources for cartilage regeneration}

Strategies for cartilage repair may involve the use of transplanted/implanted cells. The inclusion of cells adds potential to a construct in terms of production of extracellular matrix components, production of trophic factors, etc., but also poses additional challenges: sourcing of cells from autologous tissues adds additional steps to the procedure and can result in site-morbidity. Xenogeneic cell sourcing is associated with compatibility challenges and regulatory issues [77]. Additionally, the use of cells limits conditions for 3D-printing and demands handling in cell-friendly conditions [69]. The criteria for cell choice should be primarily the ability to produce tissue specific extracellular matrix, secondly, the ease of availability and the conditions of induction toward the desired phenotype [64].

Over the last two decades, multiple cell types have been evaluated for their application in cartilage repair. Autologous chondrocytes and chondrons (chondrocytes with their surrounding pericellular matrix) have been the obvious first route investigated: these cells can be harvested from non-weightbearing areas, or from the perimeter of a defect in the patient [77, 78]. Obtaining a sufficient number of chondrocytes for therapy however, requires harvest from a second site, with chances of donor site morbidity; alternatively, it requires in vitro expansion which has been shown to cause dedifferentiation of cells towards a fibroblastic phenotype [79]. Multipotent mesenchymal stromal cells (MSCs) can constitute an alternative cell source. These cells can be easily obtained from multiple tissues, including bone marrow, adipose tissue and muscles [80]. MSCs can be instructed with specific growth factors to differentiate into chondrocyte-like cells, although the right combination of cues must be provided to prevent their tendency towards differentiating into hypertrophic chondrocytes, expressing markers, such as collagen type $X$ and MMP13, indicators of endochondral ossification and eventual bone formation $[81,82]$. To address this limitation, researchers have used chondrocytes in combination with MSCs, as MSCs appear to stimulate and direct chondrocytes towards synthesis of cartilage-like tissue [83, 84].

Due to the practical limitations associated with the use of autologous tissues, alternative allogeneic sources have been evaluated for regeneration purposes. Although there are reports in literature describing how allogeneic 
transplantation of isolated chondrocytes elicits an immune response that promotes the deterioration of the repair tissue [85, 86], several studies suggest that this may not be the case for tissue engineered constructs seeded with allogeneic cells [87]. Similar findings were obtained in the equine model, where chondrocyte-collagen composites were used for resurfacing of large articular defects, and no immune-related reactions were observed in the synovial membrane, synovial fluid or subchondral bone infiltrates, possibly due to the shielding effect of the matrix on the cells [88]. The use of allogeneic chondrocytes allows to eliminate the risks related to donor site morbidity and the necessity of multiple surgical sites, although it still does not solve the tissue scarcity limitation. Additionally, it involves risks of eliciting a variable immune response and of possible transmission of infections.

The limited availability of healthy cartilage tissue even for allogeneic use, has led to the exploration of xenogeneic chondrocyte sources. In the few studies that have been reported, analysis was superficial, but results tended to be positive [87]. For example, Ramallal et al. implanted porcine chondrocytes into osteochondral defects of adult rabbits, finding a hyaline-like repair tissue after 24 weeks, without indications of a host immune response. However, no immunogenic parameters or antibody reactivity were investigated [89]. The use of xenogeneic chondrocytes may address the limitation of tissue availability, but still poses risks of immunogenicity and disease transmission.

In more recent years, new sources of cells have emerged as possible candidates for cartilage repair: these include subpopulations of progenitor cells such as articular cartilage progenitor cells (ACPCs), that appear to maintain their chondrogenic phenotype during expansion [90, 91], and induced pluripotent stem cells, which are characterized by unlimited selfrenewal capacity, can be derived from a variety of easily accessible tissues, and can be driven to differentiate into chondrocytes [92].

\section{- 3D-printing and osteochondral repair}

The emerging of 3D-printing technologies has enabled the fabrication of 3D structures based on input generated through specialized design software or extrapolated from patient imaging, thus paving the road for personalized medical treatments. Three-dimensional images can be obtained from the patient with several different techniques, including magnetic resonance imaging (MRI) and computed tomography (CT). This provides detailed images 
that can be used as blueprints for 3D-printing [93, 94]. Different 3D-printing technologies are already used in the clinical setting for various purposes. Models of anatomical structures, for example, can be used by surgeons as part of the planning of complex surgeries and allow them to practice [93]. 3Dprinting can also be used for the production of custom-made surgical tools and guides [95] or for the production of personalized implants [96].

Within the area of regenerative medicine and tissue engineering, 3D-printing can also be a useful tool to enable the fabrication of scaffolds with welldefined architecture and properties, built with different biomaterials that can range from ceramics and thermoplastic polymers to hydrogels. This is a complex process, not devoid of difficulties.

The choice of biomaterial poses the first challenge. Ideal biomaterial inks, for example, suitable for the fabrication of regenerative scaffolds, have tuneable physicochemical properties, and allow for functionalization with regenerative cues [69]. In addition, biomaterials loaded with cells for bioprinting, thus termed bioinks, should also permit cell encapsulation, while maintaining printability under cell friendly conditions [69]. The most common biomaterials used for this purpose are hydrogels, which are characterized by a high water content that mimics the cell's natural habitat, with a composition that enables the addition of biological and chemical cues to enhance and direct matrix production [97]. Although hydrogels appear very promising, their use for 3Dprinting of constructs is not without challenges, as the ideal hydrogel needs to satisfy many, sometimes conflicting requirements. For instance, providing a low polymer density to sustain cell viability and function, while at the same time displaying high viscosity and stiffness to ensure high shape fidelity post printing [98].

The replication of the architecture is also a demanding task. The production of an approximate substitute can be accomplished with different approaches, which can range from the simple introduction to gradients in structure, composition or mechanics, to the combination of biomaterials to improve function and performance, to the convergence of multiple technologies that allow the integration between heterogeneous components $[99,100]$. The advancement of 3D printing technologies and particularly the development of possibilities of convergence of different elements, have allowed the realization, in the laboratory, of great candidates for further testing. 


\section{From the petri dish to the patient}

\section{In vitro evaluation of regenerative strategies for cartilage repair}

The design and refinement of the strategy represent the first fundamental steps in the quest for regeneration of cartilage tissue (Fig. 2). To assess the potential of a regenerative strategy for cartilage repair, in vitro assessment precedes testing through in vivo models.

In vitro assessment of chondrogenic potential allows observing cell interaction in a 3D environment and represents the first step towards clinical translation. In vitro studies typically evaluate the quality and quantity of production of ECM by the implanted cells, delivery of biological cues, degradation of the hydrogel in response to proliferation, and the phenotype expressed by cells throughout the duration of the study. Common evaluation methods for cartilage in vitro studies include biochemical and histological analysis to evaluate production of sulphated glycosaminoglycans and DNA [101], as well as specific determinations of particular components such as collagen types through immunohistochemistry marker assessment [102]. Further characterizations can include gene expression of the encapsulated cells for relevant markers such as aggrecan, collagen types I, II and X, and proteoglycan IV [103].

Ideally, in vitro models should recapitulate the microenvironment of the

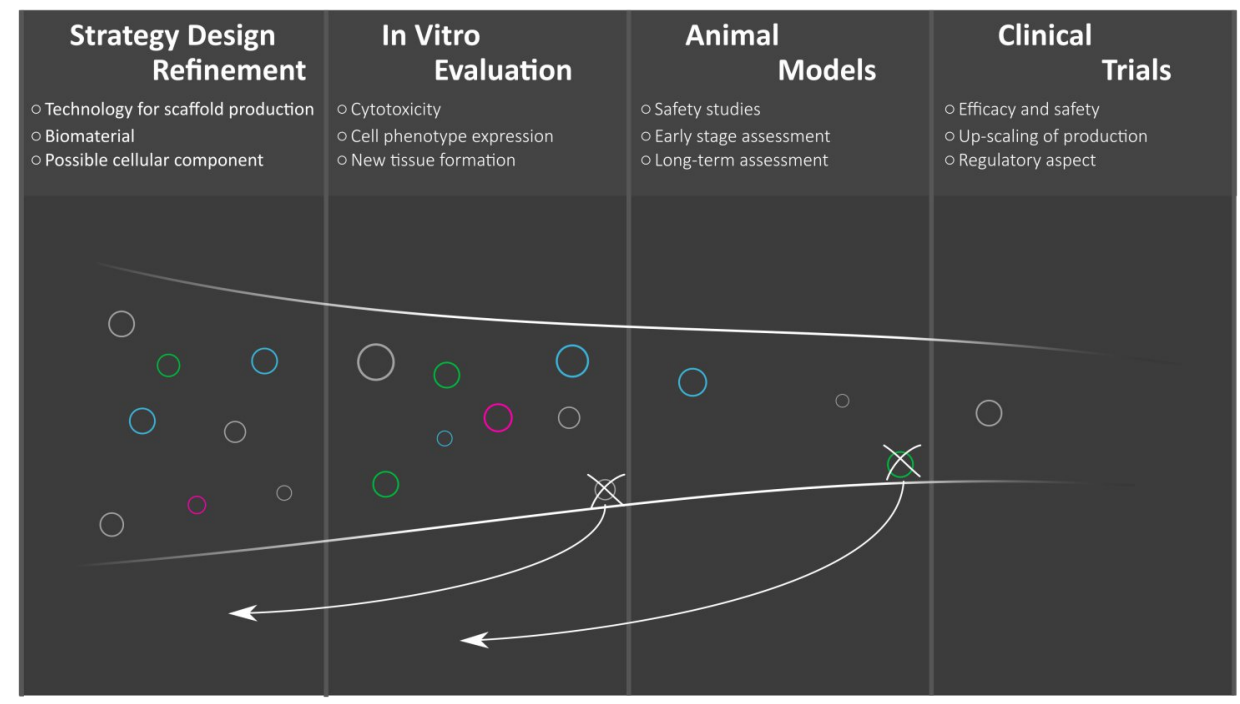

Figure 2. The steps for advancement of regenerative strategies from design to clinical trials. 
synovial joint, replicating the interactions between different cell types and components [104]. Since the joint components are influenced by their loading regimen [105], a successful model should include mechanical stimuli as they occur in nature. Since traditional in vitro culture techniques lack this possibility, a variety of advanced in vitro platforms have recently emerged. Advances in biofabrication and microfluidic technologies have resulted in platforms such as organs-on-a-chip which can provide cells with dynamic flow conditions and mechanical stimulation. These in vitro models can be adapted to simulate the native environment by combining biomaterial-based constructs with continuous perfusion, mechanical stimulation and biological and chemical cues, all within one platform [104].

\section{Functional ex vivo testing in advanced bioreactors}

The last step towards clinical translation before the (large) animal model conventionally is in vivo ectopic testing, typically in subcutaneous murine models for safety and mechanistic studies. However, animal models have a low throughput, are labour and cost-intensive and pose ethical considerations. Therefore, alternative routes are continuously investigated. Ex vivo models represent a possible route in this sense. They can be employed to pre-screen novel promising strategies, with the aim to reduce and refine the number of animals needed.

Ex vivo models can consist of cartilage explants from cadaveric joints [106]. The main limitation of these explants, is that the collagen network is discontinued during harvest, causing a change in the osmotic pressure in the cartilage tissue that leads to a loss of proteoglycans [107]. Additionally, chondral explants lack subchondral bone support. A second generation of ex vivo models consists of osteochondral explants, cultured in platforms with separate cartilage and bone medium, which allows targeted delivery of relevant biological cues without affecting the cartilage-bone interface [108]. These bioreactor systems allow the culturing of cells under controlled environmental conditions (e.g. temperature, $\mathrm{pH}$, nutrient supply) and even different mechanical stimulation regimens (tension, compression and shear) [109], moving the models closer to native tissue conditions [110]. 


\section{The equine animal model and its challenges}

While in vitro and ex vivo platforms can play a significant role in screening the potential for success of cartilage repair strategies, they are still not sufficient for the evaluation of regeneration in the clinical setting [111]. Validity of a therapy would ideally be proven by a series of independent studies involving large numbers of individuals, executed over many years. However, the need for solutions to joint degeneration is great and immediate, and therefore predictive animal models represent the next-best alternative. In theory, an animal model should match perfectly the clinical question to be addressed, while enabling evaluation of the hypothesis at hand [112].

Smaller animal models such as rabbits may offer important information for biocompatibility and early stage assessment, nonetheless they present significant differences from the human patient, such as differences in limb angulation, high cellularity and widely different biomechanical loading [113, 114].

Several large animal models have been proposed and tested for novel cartilage repair strategies and all have their limitations. Pigs have a bone structure similar to humans in terms of biochemistry and trabecular thickness. The housing of pigs, however, is cost-intensive, and requires specialized training due to the animals' temperament [115]; mini-pigs are a good alternative, particularly for short-term studies [116]. Goats and sheep have human-like anatomy of their stifle joints and are easy to keep. For longitudinal assessments though, these animals pose issues with training for consistent objective outcome measures (such as gait analysis), or for rehabilitation regimens [115].

The equine model presents the closest approximation to the human clinical situation, and is a good model for repair studies of cartilage and bone tissue. The equine model's biomechanical environment is the closest to the human situation, which allows a similarity in outcome parameters as well [113]. For example, the medial femoral condyle of the equine stifle joint, features cartilage characteristics similar to humans [114], and is ideal as a human knee osteoarthritis model [117].

In addition, the use of horses as companion and sports animals, has led to a rather thorough understanding of the joint tissue composition and the response to stress and degeneration. In horses, like in people, cartilage injuries commonly occur during high impact activities and eventually result in the 
development of osteoarthritis [118]. A large epidemiologic study conducted in the UK, in over 600 Thoroughbred racehorses for a total of 7785 months, found that 165 of these had at least one joint injury, with an overall joint injury rate of 1.8 per 100 horse months [119]. A large survey conducted by the United States Department of Agriculture reported that approximately $50 \%$ of commercial horse operations had at least one of their animals affected by lameness, and in about $50 \%$ of these cases lameness was related to a limb or joint issue [120]. The high incidence of joint diseases makes horses very good candidates for replication of human clinical conditions, while allowing simultaneously to conduct investigations for which they represent the target species [121], benefitting two species simultaneously. Other advantages of the model include joint size, which allows longitudinal monitoring with arthroscopy, ultrasound, CT and potentially MRI, and aptness to training, enabling researchers to perform repeated objective measurements such as kinetic and kinematic analyses [117]. However, the equine model poses significant biomechanical challenges, especially because of the horse's inability to unload a limb for any prolonged period of time and hence the inevitability of immediate weight-bearing post-surgery [122]. Furthermore, horses are costintensive both to acquire and maintain, and require large, specialized resources for management, housing and husbandry [117].

\section{Thesis outline and research questions}

The general aim of this thesis was to develop translational strategies for (osteo)chondral repair by refining the equine model and studying the osteochondral tissue's template. Many possibilities for repair have been investigated over the years, without successfully breaking the deadlock represented by the long-term failure of newly-formed repair tissue in cartilage defects. Therefore, the first part (part I) of this thesis is dedicated to the investigation of fundamental aspects of the osteochondral unit with the aim of identifying the key adaptations relevant for tissue engineering strategy design. The second portion (part II) focuses on the translation of promising in vitro regenerative strategies towards the preclinical equine model, refining the model by addressing key issues such as fixation of the implant. Several regenerative approaches were evaluated, starting from the implantation of a cell-laden biomaterial directly cast in a surgical setting, to a more complex approach with an advanced composite scaffold for the repair of large 
osteochondral defects.

The specific research questions addressed in this thesis are:

\section{Part I) Understanding the native osteochondral template}

Chapter 2. What is the effect of body mass on the microstructural features of the osteochondral unit? Which structures are more conserved and which vary to adapt to different loads? Research suggests that the unique biomechanical specialization of the osteochondral tissue may represent the biggest challenge in terms of replication [68]. Investigating the effect of scaling on the microstructural characteristics across a wide range of mammals helps in understanding which structures have key roles in adapting to (high) loads. This insight may be valuable for future designing of advanced regenerative strategies.

Chapter 3. What are the differences in the osteochondral unit in mammals that live with and without loading? During evolution, mammals have returned from land to water, finding different solutions for locomotion in drastically different environments. The research reported in this chapter analyses the differences in osteochondral unit features between aquatic and terrestrial mammals. These differences reflect the importance of coping with loading for proper joint function and suggest key considerations to be made when designing regenerative constructs for joint repair.

Part II) Translation of hydrogel-based regenerative strategies in the equine model for cartilage repair

Chapter 4. Can (reinforced) gelMA hydrogel be translated to the equine model and used for the repair of focal cartilage defects? Cell therapies for cartilage repair may rely on rapidly degrading cell carriers such as fibrin glue [77]. These carriers differ significantly from regenerative biomaterials such as hydrogels, as the latter have a lower degradation rate meant ideally to match that of new tissue formation $[123,124]$. Given the in vitro success shown by gelMA in supporting chondrogenesis, this study sets out to translate this regenerative strategy towards the equine model by addressing preliminary in vitro preparations, followed by ex vivo testing and a short-term pilot study. Subsequently, a long-term study is carried out for the evaluation of gelMA for 
cartilage repair in the equine model.

Chapter 5. With the advent of advanced tissue engineered constructs for chondral and osteochondral repair, how can fixation of the constructs in the preclinical equine model be addressed? A crucial issue in cartilage repair is the fixation of implants: a range of solutions has been adopted over the years; however they all have significant drawbacks, ranging from insufficient fixation to severe alteration of the architecture of implanted scaffolds [125]. The advancement of 3D-printing technologies enables the development of tissue engineered constructs with complex architectures and raises the issue of how to address their fixation. This chapter presents the results of a series of pilot studies aimed at the optimization of the fixation of hydrogel-based scaffolds in (osteo)chondral defects, from the use of commercial and autologous fibrin glue to the development of a customized fibre-reinforced hydrogel construct with a 3D-printed anchor for fixation of hydrogels in the equine model.

Chapter 6. Can a composite scaffold with a zonal cell distribution represent a valid strategy for (osteo)chondral repair? Will a zonal cell distribution outperform a non-zonal distribution in a long-term equine model? This chapter aims at investigating the validity of a zonal cell distribution as a strategy for (osteo)chondral repair. Here, a composite construct is developed utilizing an established 3D-printed anchor, combined with a hydrogel based, PCL reinforced chondral portion. The approach is first tested with a thiol-ene crosslinkable hyaluronic acid/poly(glycidol) hybrid hydrogel [126], in combination with a zonal distribution of mesenchymal stromal cells and articular cartilage progenitor cells, in a long-term study in the equine model for cartilage repair.

Chapter 7. Can a hydrogel with a longer degradation profile improve (osteo)chondral repair with a composite scaffold with a zonal cell distribution? This chapter also focuses at the validity of a zonal cell distribution as strategy for (osteo)chondral repair, but this time with a different biomaterial. A composite construct with a zonal architecture is fabricated, consisting of a 3Dprinted PCL anchor and a StarPEG/heparin hydrogel [127] characterized by a longer degradation rate than the gel used in Chapter 6, and tested for performance in a similar long-term study in the equine model for cartilage repair. 
Chapter 8. General Discussion. This chapter discusses the major findings of both the fundamental research in Part I of the thesis and the more applied work of Part II, and puts these in the wider perspective of the ongoing quest into developing an effective and durable way of regenerating articular cartilage. Based on our own findings and those of others, suggestions are given which direction this quest might take in the near future and which might be the most important criteria to guide the research. 


\section{REFERENCES}

[1] K.E. Jones, K. Safi, Ecology and evolution of mammalian biodiversity, 366 (2011) 2451-2461.

[2] K.E. Jones, J. Bielby, M. Cardillo, S.A. Fritz, J. O'Dell, C.D.L. Orme, K. Safi, W. Sechrest, E.H. Boakes, C. Carbone, PanTHERIA: a species-level database of life history, ecology, and geography of extant and recently extinct mammals: Ecological Archives E090-184, Ecology 90(9) (2009) 2648-2648.

[3] M. Shimamura, H. Yasue, K. Ohshima, H. Abe, H. Kato, T. Kishiro, M. Goto, I. Munechika, N. Okada, Molecular evidence from retroposons that whales form a clade within even-toed ungulates, Nature 388(6643) (1997) 666-670.

[4] P. Dejours, Water and air physical characteristics and their physiological consequences, Comparative physiology: Life in water and on land 9(3) (1987).

[5] T.M. Williams, The evolution of cost efficient swimming in marine mammals: limits to energetic optimization, Philosophical Transactions of the Royal Society of London. Series B: Biological Sciences 354(1380) (1999) 193-201.

[6] B. Cozzi, S. Mazzariol, M. Podestà, A. Zotti, Diving adaptations of the cetacean skeleton, The Open Zoology Journal 2(1) (2009) 24-32.

[7] A.J. Sophia Fox, A. Bedi, S.A. Rodeo, The basic science of articular cartilage: structure, composition, and function, Sports health 1(6) (2009) 461-468.

[8] R.J. Lories, F.P. Luyten, The bone-cartilage unit in osteoarthritis, Nature Reviews Rheumatology 7(1) (2011) 43.

[9] S.I. Lepage, N. Robson, H. Gilmore, O. Davis, A. Hooper, S. St. John, V. Kamesan, P. Gelis, D. Carvajal, M. Hurtig, Beyond cartilage repair: the role of the osteochondral unit in joint health and disease, Tissue Engineering Part B: Reviews 25(2) (2019) 114-125.

[10] R. Stockwell, Chondrocytes, Journal of Clinical Pathology. Supplement (Royal College of Pathologists). 12 (1978) 7.

[11] M. Ulrich-Vinther, M.D. Maloney, E.M. Schwarz, R. Rosier, R.J. O'Keefe, Articular cartilage biology, JAAOS-Journal of the American Academy of Orthopaedic Surgeons 11(6) (2003) 421-430.

[12] E.E. Coates, J.P. Fisher, Phenotypic variations in chondrocyte subpopulations and their response to in vitro culture and external stimuli, Annals of biomedical engineering 38(11) (2010) 3371-3388.

[13] V.C. Mow, A. Ratcliffe, A.R. Poole, Cartilage and diarthrodial joints as paradigms for hierarchical materials and structures, Biomaterials 13(2) (1992) 67-97.

[14] X. Lu, V. Mow, Biomechanics of articular cartilage and determination of material properties, Medicine+ Science in Sports+ Exercise 40(2) (2008) 193.

[15] I. Redler, V.C. Mow, M.L. Zimny, J. Mansell, The ultrastructure and biomechanical significance of the tidemark of articular cartilage, Clin Orthop Relat Res (112) (1975) 357-362.

[16] P. Mente, J.L. Lewis, Elastic modulus of calcified cartilage is an order of magnitude less than that of subchondral bone, Journal of Orthopaedic Research 12(5) (1994) 637-647.

[17] T.J. Lyons, S.F. McClure, R.W. Stoddart, J. McClure, The normal human chondro-osseous junctional region: evidence for contact of uncalcified cartilage with subchondral bone and marrow spaces, BMC musculoskeletal disorders 7(1) (2006) 52.

[18] H. Madry, C.N. van Dijk, M. Mueller-Gerbl, The basic science of the subchondral bone, Knee surgery, sports traumatology, arthroscopy 18(4) (2010) 419-433.

[19] T.J. Lyons, R. Stoddart, S. McClure, J. McClure, The tidemark of the chondro-osseous junction of the normal human knee joint, Journal of molecular histology 36(3) (2005) 207-215.

[20] X. Feng, Chemical and Biochemical Basis of Cell-Bone Matrix Interaction in Health and Disease, Curr Chem Biol 3(2) (2009) 189-196.

[21] A.H. Burstein, J. Zika, K. Heiple, L. Klein, Contribution of collagen and mineral to the elasticplastic properties of bone, JBJS 57(7) (1975) 956-961.

[22] S.R. Simon, E.L. Radin, I.L. Paul, R.M. Rose, The response of joints to impact loading - II In vivo behavior of subchondral bone, Journal of Biomechanics 5(3) (1972) 267-272.

[23] Y. Wolff, Das Gesetz der Transformation der Knochen (Berlin: Verlag von August Hirschwald), 
(1892).

[24] R. Huiskes, If bone is the answer, then what is the question?, The Journal of Anatomy 197(2) (2000) 145-156.

[25] P. Brama, J. TeKoppele, R. Bank, A. Barneveld, P. Van Weeren, Biochemical development of subchondral bone from birth until age eleven months and the influence of physical activity, Equine veterinary journal 34(2) (2002) 143-149.

[26] B.M. Gorissen, C.F. Wolschrijn, B. van Rietbergen, L. Rieppo, S. Saarakkala, P.R. van Weeren, Trabecular and subchondral bone development of the talus and distal tibia from foal to adult in the warmblood horse, Anatomia, histologia, embryologia 47(3) (2018) 206-215.

[27] H.L. Stewart, C.E. Kawcak, The importance of subchondral bone in the pathophysiology of osteoarthritis, Frontiers in veterinary science 5 (2018) 178.

[28] H. Brommer, P. Brama, M. Laasanen, H. Helminen, P. Van Weeren, J. Jurvelin, Functional adaptation of articular cartilage from birth to maturity under the influence of loading: a biomechanical analysis, Equine veterinary journal 37(2) (2005) 148-154.

[29] P. Brama, J. Tekoppele, R. Bank, A. Barneveld, P. Van Weeren, Functional adaptation of equine articular cartilage: the formation of regional biochemical characteristics up to age one year, Equine veterinary journal 32(3) (2000) 217-221.

[30] K.M. Heinemeier, P. Schjerling, J. Heinemeier, M.B. Møller, M.R. Krogsgaard, T. GrumSchwensen, M.M. Petersen, M. Kjaer, Radiocarbon dating reveals minimal collagen turnover in both healthy and osteoarthritic human cartilage, Science translational medicine 8(346) (2016) 346ra90-346ra90.

[31] S. Weiner, W. Traub, H.D. Wagner, Lamellar bone: structure-function relations, Journal of structural biology 126(3) (1999) 241-255.

[32] J. Wolff, Das Gesetz der Transformation der Knochen, DMW-Deutsche Medizinische Wochenschrift 19(47) (1893) 1222-1224.

[33] W. Roux, Der zuchtende Kampf der Teile, oder die "Teilauslese" im Organismus (Theorie der "funktionellen Anpassung"), Leipzig: Wilhelm Engelmann (1881).

[34] J.G. Skedros, R.A. Brand, Biographical sketch: Georg Hermann von Meyer (1815-1892), Clin Orthop Relat Res 469(11) (2011) 3072.

[35] H.M. Frost, Perspectives: A proposed general model of the "mechanostat"(suggestions from a new skeletal-biologic paradigm), The Anatomical Record: An Official Publication of the American Association of Anatomists 244(2) (1996) 139-147.

[36] H.M. Frost, From Wolff's law to the Utah paradigm: insights about bone physiology and its clinical applications, The Anatomical Record: An Official Publication of the American Association of Anatomists 262(4) (2001) 398-419.

[37] E.A. Makris, A.H. Gomoll, K.N. Malizos, J.C. Hu, K.A. Athanasiou, Repair and tissue engineering techniques for articular cartilage, Nature Reviews Rheumatology 11(1) (2015) 21.

[38] G. Sampogna, S.Y. Guraya, A. Forgione, Regenerative medicine: Historical roots and potential strategies in modern medicine, Journal of microscopy and ultrastructure 3(3) (2015) 101-107.

[39] S. Dimmeler, S. Ding, T.A. Rando, A. Trounson, Translational strategies and challenges in regenerative medicine, Nature medicine 20(8) (2014) 814.

[40] R. Langer, J. Vacanti, Tissue engineering, Science 260(5110) (1993) 920-926.

[41] S.V. Murphy, A. Atala, 3D bioprinting of tissues and organs, Nature biotechnology 32(8) (2014) 773.

[42] C.W. Archer, P. Francis-West, The chondrocyte, The international journal of biochemistry \& cell biology 35(4) (2003) 401-404.

[43] M. Dueñas, B. Ojeda, A. Salazar, J.A. Mico, I. Failde, A review of chronic pain impact on patients, their social environment and the health care system, Journal of pain research 9 (2016) 457.

[44] I. Jack Farr, L.E. Miller, J.E. Block, Quality of life in patients with knee osteoarthritis: a commentary on nonsurgical and surgical treatments, The open orthopaedics journal 7 (2013) 619.

[45] K.E. Benders, P.R. van Weeren, S.F. Badylak, D.B. Saris, W.J. Dhert, J. Malda, Extracellular matrix scaffolds for cartilage and bone regeneration, Trends in biotechnology 31(3) (2013) 169- 
176.

[46] G.J. Van Osch, M. Brittberg, J.E. Dennis, Y.M. Bastiaansen-Jenniskens, R.G. Erben, Y.T. Konttinen, F.P. Luyten, Cartilage repair: past and future-lessons for regenerative medicine, Journal of cellular and molecular medicine 13(5) (2009) 792-810.

[47] J. Buckwalter, H. Mankin, Articular cartilage: degeneration and osteoarthritis, repair, regeneration, and transplantation, Instructional course lectures 47 (1998) 487-504.

[48] S.C. Mastbergen, D.B. Saris, F.P. Lafeber, Functional articular cartilage repair: here, near, or is the best approach not yet clear?, Nature reviews rheumatology 9(5) (2013) 277.

[49] J.-M. Denoix, L. Jeffcott, C. Mcllwraith, P. Van Weeren, A review of terminology for equine juvenile osteochondral conditions (JOCC) based on anatomical and functional considerations, The Veterinary Journal 197(1) (2013) 29-35.

[50] J.M. Flynn, M.S. Kocher, T.J. Ganley, Osteochondritis dissecans of the knee, Journal of Pediatric Orthopaedics 24(4) (2004) 434-443.

[51] L. Andriolo, D.C. Crawford, D. Reale, S. Zaffagnini, C. Candrian, A. Cavicchioli, G. Filardo, Osteochondritis dissecans of the knee: etiology and pathogenetic mechanisms. A systematic review, Cartilage (2018) 1947603518786557.

[52] S. Grässel, J. Lorenz, Tissue-engineering strategies to repair chondral and osteochondral tissue in osteoarthritis: use of mesenchymal stem cells, Current rheumatology reports 16(10) (2014) 452.

[53] I. Haq, E. Murphy, J. Dacre, Osteoarthritis, Postgrad Med J 79 (2003) 377-383.

[54] World Health Organization, Department of Chronic Diseases and Health Promotion. https:// www.who.int/chp/topics/rheumatic/en/. (Accessed 2906 2020).

[55] E.M.C.S.a.l. Network, Musculoskeletal Health in Europe: Report v5.0, in: E.M.C.S.a.I. Network (Ed.) 2012.

[56] B.R. Mandelbaum, J.E. Browne, F. Fu, L. Micheli, J.B. Mosely, C. Erggelet, T. Minas, L. Peterson, Articular cartilage lesions of the knee, The American journal of sports medicine 26(6) (1998) 853-861.

[57] D.G. Jones, L. Peterson, Autologous chondrocyte implantation, JBJS 88(11) (2006) 25012520.

[58] D.B. Saris, J. Vanlauwe, J. Victor, M. Haspl, M. Bohnsack, Y. Fortems, B. Vandekerckhove, K.F. Almqvist, T. Claes, F. Handelberg, Characterized chondrocyte implantation results in better structural repair when treating symptomatic cartilage defects of the knee in a randomized controlled trial versus microfracture, The American journal of sports medicine 36(2) (2008) 235246.

[59] R. Gudas, R.J. Kalesinskas, V. Kimtys, E. Stankevičius, V. Toliušis, G. Bernotavičius, A. Smailys, A prospective randomized clinical study of mosaic osteochondral autologous transplantation versus microfracture for the treatment of osteochondral defects in the knee joint in young athletes, Arthroscopy: The Journal of Arthroscopic \& Related Surgery 21(9) (2005) 1066-1075.

[60] M. Petri, M. Ettinger, C. Von Falck, N. Hawi, M. Jagodzinski, C. Haasper, Reconstruction of osteochondral defects by combined bone grafting and a bilayer collagen membrane as a sandwich technique, Orthopedic reviews 5(4) (2013).

[61] T. Minas, T. Ogura, J. Headrick, T. Bryant, Autologous chondrocyte implantation "sandwich" technique compared with autologous bone grafting for deep osteochondral lesions in the knee, The American journal of sports medicine 46(2) (2018) 322-332.

[62] R. Andrade, S. Vasta, R. Pereira, H. Pereira, R. Papalia, M. Karahan, J.M. Oliveira, R.L. Reis, J. Espregueira-Mendes, Knee donor-site morbidity after mosaicplasty-a systematic review, Journal of experimental orthopaedics 3(1) (2016) 31.

[63] S. Panseri, A. Russo, C. Cunha, A. Bondi, A. Di Martino, S. Patella, E. Kon, Osteochondral tissue engineering approaches for articular cartilage and subchondral bone regeneration, Knee Surgery, Sports Traumatology, Arthroscopy 20(6) (2012) 1182-1191.

[64] D.J. Huey, J.C. Hu, K.A. Athanasiou, Unlike Bone, Cartilage Regeneration Remains Elusive, Science 338(6109) (2012) 917-921.

[65] D. Tang, R.S. Tare, L.-Y. Yang, D.F. Williams, K.-L. Ou, R.O. Oreffo, Biofabrication of bone tissue: approaches, challenges and translation for bone regeneration, Biomaterials 83 (2016) 
363-382.

[66] U. Meyer, H.P. Wiesmann, Bone and cartilage engineering, Springer Science \& Business Media2006.

[67] A. Armiento, M. Stoddart, M. Alini, D. Eglin, Biomaterials for articular cartilage tissue engineering: Learning from biology, Acta biomaterialia 65 (2018) 1-20.

[68] J. Malda, J. Groll, P.R. van Weeren, Rethinking articular cartilage regeneration based on a 250-year-old statement, Nature Reviews Rheumatology (15) (2019) 571-572.

[69] J. Malda, J. Visser, F.P. Melchels, T. Jüngst, W.E. Hennink, W.J. Dhert, J. Groll, D.W. Hutmacher, 25th anniversary article: engineering hydrogels for biofabrication, Advanced materials 25(36) (2013) 5011-5028.

[70] B.J. Klotz, D. Gawlitta, A.J. Rosenberg, J. Malda, F.P. Melchels, Gelatin-methacryloyl hydrogels: towards biofabrication-based tissue repair, Trends in biotechnology 34(5) (2016) 394407.

[71] J.S. Temenoff, A.G. Mikos, Review: tissue engineering for regeneration of articular cartilage, Biomaterials 21(5) (2000) 431-440.

[72] D. Puppi, F. Chiellini, A.M. Piras, E. Chiellini, Polymeric materials for bone and cartilage repair, Progress in Polymer Science 35(4) (2010) 403-440.

[73] F.P. Melchels, M.A. Domingos, T.J. Klein, J. Malda, P.J. Bartolo, D.W. Hutmacher, Additive manufacturing of tissues and organs, Progress in Polymer Science 37(8) (2012) 1079-1104.

[74] D. Varghese, M. Deshpande, T. Xu, P. Kesari, S. Ohri, T. Boland, Advances in tissue engineering: cell printing, The Journal of thoracic and cardiovascular surgery 129(2) (2005) 470472.

[75] J. Visser, F.P. Melchels, J.E. Jeon, E.M. Van Bussel, L.S. Kimpton, H.M. Byrne, W.J. Dhert, P.D. Dalton, D.W. Hutmacher, J. Malda, Reinforcement of hydrogels using three-dimensionally printed microfibres, Nature communications 6(1) (2015) 1-10.

[76] J.M. Coburn, M. Gibson, S. Monagle, Z. Patterson, J.H. Elisseeff, Bioinspired nanofibers support chondrogenesis for articular cartilage repair, Proceedings of the National Academy of Sciences 109(25) (2012) 10012-10017.

[77] J.E. Bekkers, A.I. Tsuchida, M.H. van Rijen, L.A. Vonk, W.J. Dhert, L.B. Creemers, D.B. Saris, Single-stage cell-based cartilage regeneration using a combination of chondrons and mesenchymal stromal cells: comparison with microfracture, The American journal of sports medicine 41(9) (2013) 2158-2166.

[78] Z. Zhang, Chondrons and the pericellular matrix of chondrocytes, Tissue Engineering Part B: Reviews 21(3) (2015) 267-277.

[79] B. Ma, J.C.H. Leijten, L. Wu, M. Kip, C. van Blitterswijk, J.N. Post, M. Karperien, Gene expression profiling of dedifferentiated human articular chondrocytes in monolayer culture, Osteoarthritis and cartilage 21(4) (2013) 599-603.

[80] M. Dominici, K. Le Blanc, I. Mueller, I. Slaper-Cortenbach, F. Marini, D. Krause, R. Deans, A. Keating, D. Prockop, E. Horwitz, Minimal criteria for defining multipotent mesenchymal stromal cells. The International Society for Cellular Therapy position statement, Cytotherapy 8(4) (2006) 315-317.

[81] J. Visser, D. Gawlitta, K.E. Benders, S.M. Toma, B. Pouran, P.R. van Weeren, W.J. Dhert, J. Malda, Endochondral bone formation in gelatin methacrylamide hydrogel with embedded cartilage-derived matrix particles, Biomaterials 37 (2015) 174-182.

[82] K. Pelttari, A. Winter, E. Steck, K. Goetzke, T. Hennig, B.G. Ochs, T. Aigner, W. Richter, Premature induction of hypertrophy during in vitro chondrogenesis of human mesenchymal stem cells correlates with calcification and vascular invasion after ectopic transplantation in SCID mice, Arthritis \& Rheumatism 54(10) (2006) 3254-3266.

[83] T.S. de Windt, D.B. Saris, I.C. Slaper-Cortenbach, M.H. van Rijen, D. Gawlitta, L.B. Creemers, R.A. de Weger, W.J. Dhert, L.A. Vonk, Direct cell-cell contact with chondrocytes is a key mechanism in multipotent mesenchymal stromal cell-mediated chondrogenesis, Tissue Engineering Part A 21(19-20) (2015) 2536-2547.

[84] V.H. Mouser, R. Levato, L.J. Bonassar, D.D. D'Lima, D.A. Grande, T.J. Klein, D.B. Saris, M. Zenobi-Wong, D. Gawlitta, J. Malda, Three-dimensional bioprinting and its potential in the field 
of articular cartilage regeneration, Cartilage 8(4) (2017) 327-340.

[85] J. Malejczyk, S. Moskalewski, Effect of immunosuppression on survival and growth of cartilage produced by transplanted allogeneic epiphyseal chondrocytes, Clin Orthop Relat Res (232) (1988) 292-303.

[86] J. Malejczyk, A. Osieka, A. Hyc, S. Moskalewski, Effect of immunosuppression on rejection of cartilage formed by transplanted allogeneic rib chondrocytes in mice, Clin Orthop Relat Res 269 (1991) 266-273.

[87] C.M. Revell, K.A. Athanasiou, Success rates and immunologic responses of autogenic, allogenic, and xenogenic treatments to repair articular cartilage defects, Tissue Eng. Part B Rev. 15(1) (2009) 1-15.

[88] A.E. Sams, A.J. Nixon, Chondrocyte-laden collagen scaffolds for resurfacing extensive articular cartilage defects, Osteoarthritis Cartilage 3(1) (1995) 47-59.

[89] M. Ramallal, E. Maneiro, E. López, I. Fuentes-Boquete, M.J. López-Armada, J.L. FernándezSueiro, F. Galdo, F.J. De Toro, F.J. Blanco, Xeno-implantation of pig chondrocytes into rabbit to treat localized articular cartilage defects: An animal model, Wound Repair Regen. 12(3) (2004) 337-345.

[90] Y. Jiang, R.S. Tuan, Origin and function of cartilage stem/progenitor cells in osteoarthritis, Nature Reviews Rheumatology 11(4) (2015) 206.

[91] G.P. Dowthwaite, J.C. Bishop, S.N. Redman, I.M. Khan, P. Rooney, D.J. Evans, L. Haughton, Z. Bayram, S. Boyer, B. Thomson, The surface of articular cartilage contains a progenitor cell population, Journal of cell science 117(6) (2004) 889-897.

[92] S.P. Medvedev, E.V. Grigor'eva, A.I. Shevchenko, A.A. Malakhova, E.V. Dementyeva, A.A. Shilov, E.A. Pokushalov, A.M. Zaidman, M.A. Aleksandrova, E.Y. Plotnikov, Human induced pluripotent stem cells derived from fetal neural stem cells successfully undergo directed differentiation into cartilage, Stem cells and development 20(6) (2011) 1099-1112.

[93] B. Spottiswoode, D. Van den Heever, Y. Chang, S. Engelhardt, S. Du Plessis, F. Nicolls, H. Hartzenberg, A. Gretschel, Preoperative three-dimensional model creation of magnetic resonance brain images as a tool to assist neurosurgical planning, Stereotactic and functional neurosurgery 91(3) (2013) 162-169.

[94] Y.-X. Zheng, D.-F. Yu, J.-G. Zhao, Y.-L. Wu, B. Zheng, 3D printout models vs. 3D-rendered images: which is better for preoperative planning?, Journal of surgical education 73(3) (2016) 518-523.

[95] J.W. Noble Jr, C.A. Moore, N. Liu, The value of patient-matched instrumentation in total knee arthroplasty, The Journal of arthroplasty 27(1) (2012) 153-155.

[96] A. Mazzoli, Selective laser sintering in biomedical engineering, Medical \& biological engineering \& computing 51(3) (2013) 245-256.

[97] D. Seliktar, Designing cell-compatible hydrogels for biomedical applications, Science 336(6085) (2012) 1124-1128.

[98] S. Khalil, W. Sun, Bioprinting endothelial cells with alginate for 3D tissue constructs, Journal of biomechanical engineering 131(11) (2009).

[99] W.M. Groen, P. Diloksumpan, P.R. van Weeren, R. Levato, J. Malda, From intricate to integrated: Biofabrication of articulating joints, Journal of Orthopaedic Research 35(10) (2017) 2089-2097.

[100] M. de Ruijter, A. Ribeiro, I. Dokter, M. Castilho, J. Malda, Simultaneous micropatterning of fibrous meshes and bioinks for the fabrication of living tissue constructs, Advanced healthcare materials 8(7) (2019) 1800418.

[101] M.L. Gutiérrez, J. Guevara, L.A. Barrera, Semi-automatic grading system in histologic and immunohistochemistry analysis to evaluate in vitro chondrogenesis, Universitas Scientiarum 17(2) (2012) 167-178.

[102] P.A. Zuk, M. Zhu, P. Ashjian, D.A. De Ugarte, J.I. Huang, H. Mizuno, Z.C. Alfonso, J.K. Fraser, P. Benhaim, M.H. Hedrick, Human adipose tissue is a source of multipotent stem cells, Molecular biology of the cell 13(12) (2002) 4279-4295.

[103] R. Levato, W.R. Webb, I.A. Otto, A. Mensinga, Y. Zhang, M. van Rijen, R. van Weeren, I.M. Khan, J. Malda, The bio in the ink: cartilage regeneration with bioprintable hydrogels and 
articular cartilage-derived progenitor cells, Acta biomaterialia 61 (2017) 41-53.

[104] S. Piluso, Y. Li, F. Abinzano, R. Levato, L.M. Teixeira, M. Karperien, J. Leijten, R. van Weeren, J. Malda, Mimicking the articular joint with in vitro models, Trends in biotechnology $37(10)$ (2019) 1063-1077.

[105] H.B. Sun, Mechanical loading, cartilage degradation, and arthritis, Annals of the New York Academy of Sciences 1211(1) (2010) 37-50.

[106] S. Sharma, N. Vazquez-Portalatin, S. Calve, A. Panitch, Biomimetic molecules lower catabolic expression and prevent chondroitin sulfate degradation in an osteoarthritic ex vivo model, ACS biomaterials science \& engineering 2(2) (2016) 241-250.

[107] S. Bolis, C.J. Handley, W.D. Cornper, Passive loss of proteoglycan from articular cartilage explants, Biochimica et Biophysica Acta (BBA)-General Subjects 993(2-3) (1989) 157-167.

[108] A. Schwab, A. Meeuwsen, F. Ehlicke, J. Hansmann, L. Mulder, A. Smits, H. Walles, L. Kock, Ex vivo culture platform for assessment of cartilage repair treatment strategies, ALTEX-Alternatives to animal experimentation 34(2) (2017) 267-277.

[109] S. Grad, D. Eglin, M. Alini, M.J. Stoddart, Physical stimulation of chondrogenic cells in vitro: a review, Clin Orthop Relat Res 469(10) (2011) 2764-2772.

[110] G.M. Salzmann, M.J. Stoddart, Bioreactor Tissue Engineering for Cartilage Repair, in: P.J. Emans, L. Peterson (Eds.), Developing Insights in Cartilage Repair, Springer London, London, 2014, pp. 79-97.

[111] M.B. Hurtig, M.D. Buschmann, L.A. Fortier, C.D. Hoemann, E.B. Hunziker, J.S. Jurvelin, P. Mainil-Varlet, C.W. Mcllwraith, R.L. Sah, R.A. Whiteside, Preclinical studies for cartilage repair: recommendations from the International Cartilage Repair Society, Cartilage 2(2) (2011) 137-152.

[112] G. Reinholz, L. Lu, D. Saris, M.J. Yaszemski, S. O'driscoll, Animal models for cartilage reconstruction, Biomaterials 25(9) (2004) 1511-1521.

[113] J. Cook, C. Hung, K. Kuroki, A. Stoker, C. Cook, F. Pfeiffer, S. Sherman, J. Stannard, Animal models of cartilage repair, Bone \& joint research 3(4) (2014) 89-94.

[114] J. Malda, K. Benders, T. Klein, J. De Grauw, M. Kik, D. Hutmacher, D. Saris, P. Van Weeren, W. Dhert, Comparative study of depth-dependent characteristics of equine and human osteochondral tissue from the medial and lateral femoral condyles, Osteoarthritis and cartilage 20(10) (2012) 1147-1151.

[115] L. Goebel, L. Gao, H. Madry, Animal Models in Cartilage Repair, in: S. Grässel, A. Aszódi (Eds.), Cartilage: Volume 3: Repair Strategies and Regeneration, Springer International Publishing, Cham, 2017, pp. 189-206.

[116] M.B. Fisher, N.S. Belkin, A.H. Milby, E.A. Henning, M. Bostrom, M. Kim, C. Pfeifer, G. Meloni, G.R. Dodge, J.A. Burdick, Cartilage repair and subchondral bone remodeling in response to focal lesions in a mini-pig model: implications for tissue engineering, Tissue Engineering Part $A$ 21(3-4) (2015) 850-860.

[117] C.W. Mcllwraith, L.A. Fortier, D.D. Frisbie, A.J. Nixon, Equine models of articular cartilage repair, Cartilage 2(4) (2011) 317-326.

[118] C. Mcllwraith, D. Frisbie, C. Kawcak, The horse as a model of naturally occurring osteoarthritis, Bone \& joint research 1(11) (2012) 297-309.

[119] S. Reed, B. Jackson, C. Mc Ilwraith, I. Wright, R. Pilsworth, S. Knapp, J. Wood, J. Price, K. Verheyen, Descriptive epidemiology of joint injuries in Thoroughbred racehorses in training, Equine Vet. J. 44(1) (2012) 13-19.

[120] USDA, Lameness and Laminitis in U.S. Horses, in: USDA:APHIS:VS, National Health Monitoring System (Ed.) Fort Collins, CO, 2000.

[121] R. Williams, L. Harkins, C. Hammond, J. Wood, Racehorse injuries, clinical problems and fatalities recorded on British racecourses from flat racing and National Hunt racing during 1996, 1997 and 1998, Equine veterinary journal 33(5) (2001) 478-486.

[122] C.J. Moran, A. Ramesh, P.A. Brama, J.M. O'Byrne, F.J. O'Brien, T.J. Levingstone, The benefits and limitations of animal models for translational research in cartilage repair, Journal of experimental orthopaedics 3(1) (2016) 1.

[123] N.C. Hunt, L.M. Grover, Cell encapsulation using biopolymer gels for regenerative medicine, Biotechnology letters 32(6) (2010) 733-742. 
[124] K.L. Spiller, S.A. Maher, A.M. Lowman, Hydrogels for the repair of articular cartilage defects, Tissue engineering part B: reviews 17(4) (2011) 281-299.

[125] J. Bekkers, A. Tsuchida, J. Malda, L. Creemers, R. Castelein, D.B. Saris, W. Dhert, Quality of scaffold fixation in a human cadaver knee model, Osteoarthritis and cartilage 18(2) (2010) 266272.

[126] S. Stichler, T. Böck, N. Paxton, S. Bertlein, R. Levato, V. Schill, W. Smolan, J. Malda, J. Teßmar, T. Blunk, Double printing of hyaluronic acid/poly (glycidol) hybrid hydrogels with poly $(\varepsilon-$ caprolactone) for MSC chondrogenesis, Biofabrication 9(4) (2017) 044108.

[127] E. Hesse, U. Freudenberg, T. Niemietz, C. Greth, M. Weisser, S. Hagmann, M. Binner, C. Werner, W. Richter, Peptide-functionalized starPEG/heparin hydrogels direct mitogenicity, cell morphology and cartilage matrix distribution in vitro and in vivo, Journal of tissue engineering and regenerative medicine 12(1) (2018) 229-239. 


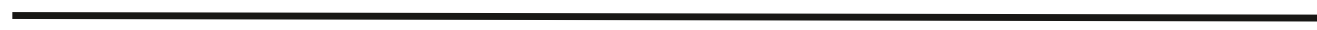




\section{Understanding the native osteochondral template}




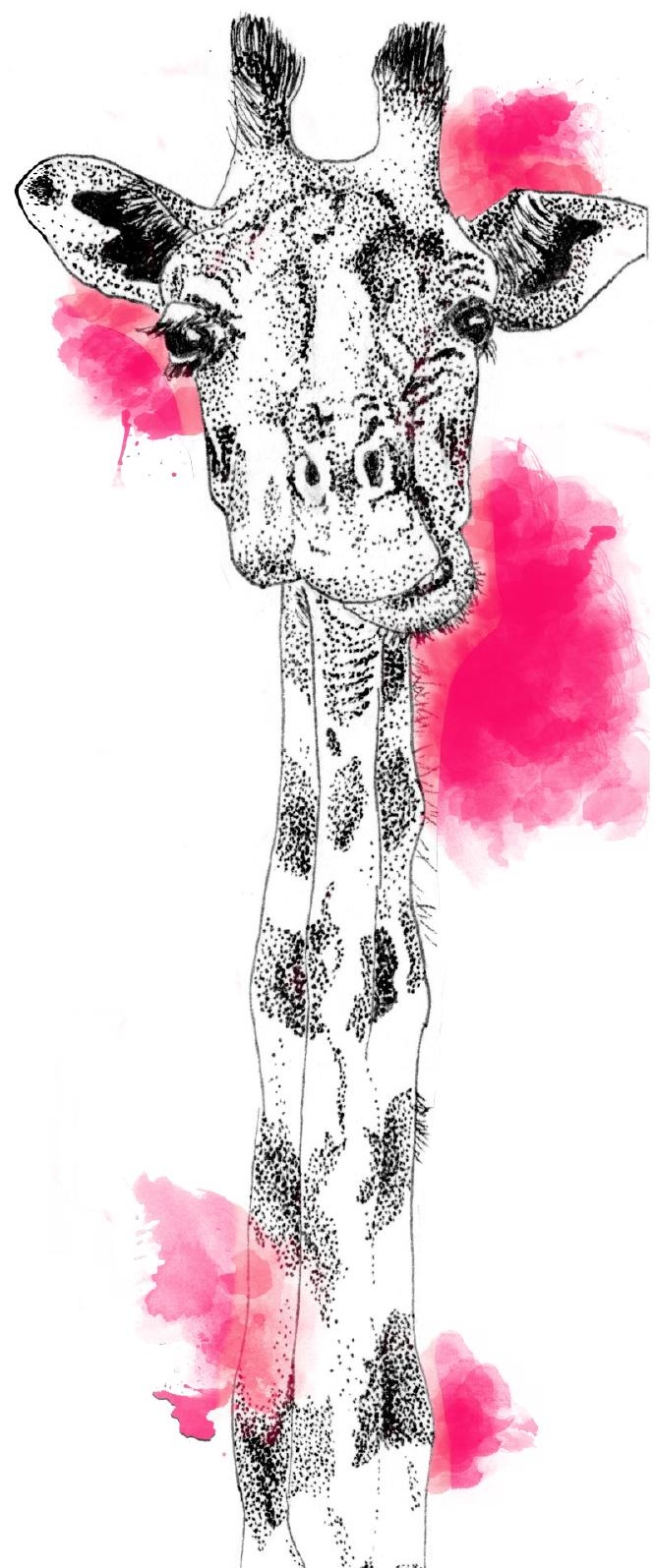




\section{Chapter}

Effects of body mass on microstructural features of the osteochondral unit: a comparative analysis of 37 mammalian species

I.A.D. Mancini

L. Rieppo

B. Pouran

I. O. Afara

F. M. Serra Braganca

M.H.P. van Rijen

M. Kik

H. Weinans

J. Töyräs

P. R. van Weeren

J. Malda

Bone, 2019, 127:664-673 


\section{ABSTRACT}

Since Galileo's days the effect of size on the anatomical characteristics of the structural elements of the body has been a subject of interest. However, the effects of scaling at tissue level have received little interest and virtually no data exist on the subject with respect to the osteochondral unit in the joint, despite this being one of the most lesion-prone and clinically relevant parts of the musculoskeletal system.

Imaging techniques, including Fourier transform infrared imaging, polarized light microscopy and micro computed tomography, were combined to study the response to increasing body mass of the osteochondral unit. We analyzed the effect of scaling on structural characteristics of articular cartilage, subchondral plate and the supporting trabecular bone, across a wide range of mammals at microscopic level.

We demonstrated that, while total cartilage thickness scales to body mass in a negative allometric fashion, thickness of different cartilage layers did not. Cartilage tissue layers were found to adapt to increasing loads principally in the deep zone with the superficial layers becoming relatively thinner. Subchondral plate thickness was found to have no correlation to body mass, nor did bone volume fraction. The underlying trabecular bone was found to have thicker trabeculae $(r=0.75, p<0.001)$, as expected since this structure carries most loads and plays a role in force mitigation.

The results of this study suggest that the osteochondral tissue structure has remained remarkably preserved across mammalian species during evolution, and that in particular, the trabecular bone carries the adaptation to the increasing body mass.

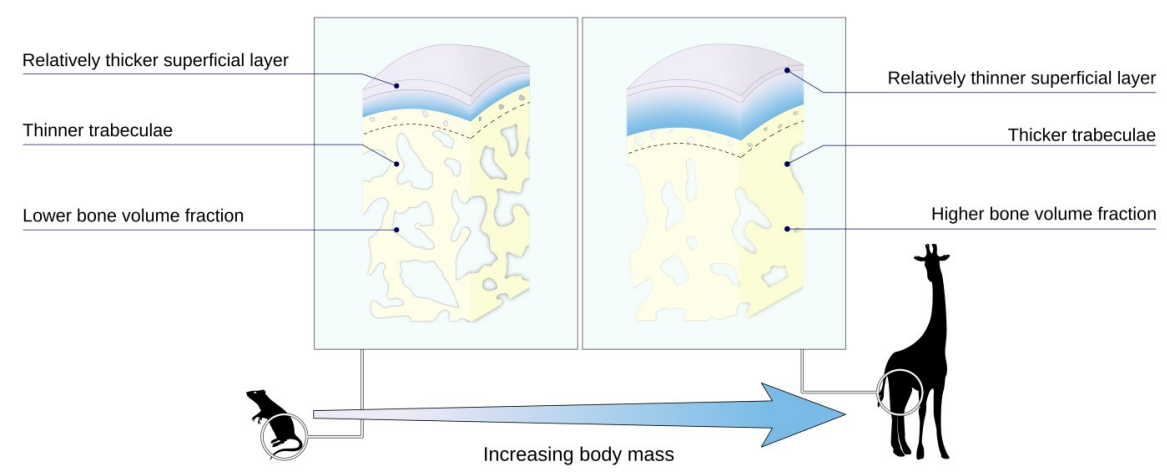




\section{INTRODUCTION}

Almost 400 years ago, after visiting Venice's Arsenal, Galileo laid the foundations to modern deformable body mechanics, by starting a discussion about scaling [1]. He took inspiration from shipbuilding and wondered how structural components would scale in bigger ships to avoid collapsing under excessive weight. This led to the formulation of the Square-Cube law that Galileo formulated as "the ratio of two volumes is greater than the ratio of their surfaces" [1]. In other words, when an object undergoes a proportional increase in size, its surface area is proportional to the square of the multiplier, while its volume is proportional to the cube of the multiplier. In addition, it also means that the stress on a larger cube is greater than the stress on a smaller cube due to its own weight [2]. Later, in the Discourses and Mathematical Demonstrations Relating to Two New Sciences, Galileo applied the law to living beings and deduced that animals could not be simply scaled up, or their bones would break under excessive weight [1]. Since then, macroscopic scaling of limbs and their components has been discussed extensively in literature [3-6]. Nevertheless, investigations on the microscopic level have been limited [7-10]. In particular, the adaptations to loading of the ensemble of the articular components (i.e. subchondral bone, cartilage and their interface) at microscopic level have never been analyzed in a comparative fashion across a large range of species.

The general structure and organization of diarthrodial joints is similar in all mammalian species. The function of these diarthrodial joints is both to minimize friction of the articulating bony components of the skeleton and to accommodate and mitigate the substantial biomechanical forces that are generated by locomotion. Hence, joint function requires its elements to provide excellent lubrication between articulating surfaces, allow force transmission and absorption, to mitigate the effects of acceleration, vibrations and peak forces generated by locomotion. To accomplish these tasks, joint components work in synergy and should be considered as a unit [11, 12], composed of articular cartilage, the subchondral bone plate and trabecular bone rather than as individual components.

Basic biochemistry, biomechanics and morphological characteristics of the major components of diarthrodial joints (i.e. hyaline articular cartilage and bone) have been studied frequently in relation to pathological changes and 
effectiveness of different treatments [12-15]. These studies, however, are usually focused on humans and animal species that are of interest as models for orthopedic research in a translational sense $[15,16]$. In nature, the spectrum of sizes and body weights in mammals is much wider than in the few species used as animal models for musculo-skeletal diseases [7, 17]. We have previously shown in a study over a wide range of species that articular width in the stifle (knee) joint scales isometrically with body mass [7]; this isometric relation can be mathematically described as $y=b x^{a}$, with $a=0.33$ [7]. If we assume that joint form is not essentially influenced by size, joint surface will do the same [18]. If then the microscopic configuration of the osteochondral unit would remain the same, the stress in the unit would increase linearly with weight given the Square-Cube law. Both articular cartilage and bone increase in size with body mass, and isolated studies on these two tissues have shown that they do not scale isometrically, but have a negative allometric relationship with increasing body mass [7-9] and therefore do not fully compensate for increasing body mass. In theory, an increase in loading can also be compensated for by changes in composition of the constituting elements of the osteochondral unit (that would possibly influence strength of the structure). However, previous research comparing articular cartilage biochemical composition across a variety of mammalian species covering a range of body masses revealed that gross biochemical composition was constant [7]. The composition and structure of articular cartilage, however, does change with depth, so that three layers (superficial, middle and deep) can be identified, based on compositional characteristics like proteoglycans and collagen content, and structural characteristics like collagen orientation. In this last case, fibrils are oriented parallel to the articular surface in the superficial zones, and transition through a random orientation to the deep zone in which they are oriented perpendicular to the subchondral bone [19].

The current study aims to comprehensively investigate the microstructural and compositional features of the osteochondral unit (across a wide range of terrestrial mammals) and their relationship to each other and to body mass (BM). This will reveal where the adaptations to increasing loads (and BM) [20] reside and will determine which microscopic features follow isometric scaling and which do not. It was hypothesized that in articular cartilage all layers would scale with negative allometry, as found earlier for total thickness [7], and that increased load would be accommodated by either structural 
adaptations in the subchondral plate or the trabecular subchondral bone, or by adaptations of the components in one or more of these layers.

\section{MATERIALS AND METHODS}

To investigate spatial biochemical composition of single layers of cartilage, Fourier-Transform Infrared Imaging (FTIRI) [21-23] was employed, allowing to determine relative content of proteoglycans (PG) and collagen by measuring absorption of specific peaks [24]. To evaluate the orientation and distribution of the cartilaginous collagen network, Polarized Light Microscopy (PLM) was chosen for its capacity to visualize the orientation of anisotropic materials [2527]. Finally, for the detailed analysis of the microstructural features of the subchondral and trabecular bone, micro computed tomography (micro-CT) was selected for the accurate measurement of micron-sized structures that constitute the bony tissue $[28,29]$.

\section{Collection of materials and tissue harvest}

Osteochondral tissue cylinders of $6 \mathrm{~mm}$ in diameter were harvested postmortem from the weight bearing central area of the medial femoral condyles of adult animals sent for autopsy to the Department of Pathobiology, Faculty of Veterinary Medicine, Utrecht University, The Netherlands. Animal species, age and body mass were recorded, and macroscopic pictures of the joints were taken. Joints demonstrating macroscopic or microscopic signs of cartilage degeneration were excluded; animals displaying signs of incomplete endochondral ossification were identified as immature and excluded as well. Human tissue samples were obtained from the Department of Pathology, University Medical Center Utrecht, The Netherlands, with approval of the local ethical committee, in line with the Dutch code of conduct for "Proper Secondary Use of Human Tissue".

In total 82 tissue samples ( 38 for histological analysis, 44 for micro-CT) were harvested from mammals belonging to 37 different species; of 38 histological samples, 5 samples could not be measured with PLM and 7 samples could not be measured with FTIRI (Table 1). Due to limited amount of tissue available, measurement with micro-CT of trabecular parameters of 4 of the 44 samples (Table 1) was not possible. Samples for histology were fixed in formalin $4 \%$, while samples for micro-CT analysis were stored in $70 \%$ ethanol; all samples 


\begin{tabular}{|c|c|c|c|c|c|}
\hline & Species & Average body mass (kg) & $\operatorname{PLM}(n)$ & FTIRI (n) & Micro-CT (n) \\
\hline 1 & Mouse (Mus musculus) & 0.03 & & & 3 \\
\hline 2 & Rat (Rattus sp) & 0.3 & 2 & 2 & 3 \\
\hline 3 & Ferret (Mustela putorius furo) & 2 & 1 & 1 & \\
\hline 4 & Hare (Lepus sp) & 3.15 & 2 & 2 & \\
\hline 5 & South American coati (Nasua nasua) & 3 & & & 1 \\
\hline 6 & Linnaeus's two-toed sloth (Choloepus didactylus) & 6.5 & 1 & 1 & \\
\hline 7 & Barbary macaque (Macaca sylvanus) & 8.5 & & & 2 \\
\hline 8 & European Badger (Meles meles) & 10 & 1 & 1 & \\
\hline 9 & Kirk's dik-dik (Madoqua kirkii) & 10 & 1 & 1 & \\
\hline 10 & Tammar wallaby (Macropus eugenii) & 12.5 & 2 & 2 & 2 \\
\hline 11 & Indian crested porcupine (Hystrix indica) & 16 & 1 & 1 & s \\
\hline 12 & Hamadryas baboon (Papio hamadryas) & 17 & & & 2 \\
\hline 13 & Roe deer (Capreolus capreolus) & 17 & 2 & 2 & $3(2)$ \\
\hline 14 & Thomson's gazelle (Eudorcas thomsonii) & 18 & 3 & 3 & $3(2)$ \\
\hline 15 & Dwarf goat (Capra aegragus hircus) & 28 & 1 & 1 & 1 \\
\hline 16 & Cheetah (Acinonyx jubatus) & 39 & & & 3 \\
\hline 17 & Impala (Aepyceros melampus) & 41 & 1 & 1 & 2 \\
\hline 18 & Red kangaroo (Macropus rufus) & 55 & 2 & 2 & 2 \\
\hline 19 & Human (Homo sapiens) & 65 & 1 & 1 & \\
\hline 20 & Fallow deer (Dama dama) & 70 & 1 & 1 & 1 \\
\hline 21 & Gorilla (Gorilla gorilla) & 80 & & & 1 \\
\hline 22 & Siberian tiger (Panthera tigris) & 80 & & & 1 \\
\hline 23 & Reindeer (Rangifer tarandus) & 125 & 1 & 1 & \\
\hline 24 & Lion (Panthera leo) & 148 & & & 1 \\
\hline 25 & Greater Kudu (Tragelaphus strepsiceros) & 150 & & & \\
\hline 26 & Llama (Lama glama) & 160 & 1 & 1 & \\
\hline 27 & Polar bear (Ursus maritimus) & 175 & & & 1 \\
\hline 28 & South American tapir (Tapirus terrestris) & 250 & & & 1 \\
\hline 29 & Elk (Alces alces) & 343 & 1 & 1 & 1 \\
\hline 30 & Watussi (Bos taurus taurus watussi) & 350 & 1 & & \\
\hline 31 & Dairy cow (Bos taurus) & 450 & 1 & 1 & \\
\hline 32 & Rothschild's giraffe (Giraffa camelopardalis) & 470 & 1 & 1 & $2(1)$ \\
\hline 33a & Shetland pony (Equus ferus caballus) & 150 & 1 & 1 & \\
\hline 33b & Horse (Equus ferus caballus) & 550 & 3 & 2 & $3(2)$ \\
\hline 34 & Banteng (Bos javanicus) & 600 & 1 & 1 & 1 \\
\hline 35 & White rhinoceros (Ceratotherium simum) & 1400 & & & 1 \\
\hline 36 & Asian elephant (Elephas maximus) & 3350 & & & 2 \\
\hline 37 & African elephant (Loxodonta africana) & 4000 & & & 1 \\
\hline Total & & & 33 & 31 & $44(40)$ \\
\hline
\end{tabular}

Table 1. List of species included in the study. In total 82 tissue samples (38 for histological analysis, 44 for micro-CT) were harvested from mammals belonging to 37 different species; of the 38 histological samples, 33 were measured with PLM and 31 were measured with FTIRI. Fortyfour samples were measured with micro-CT, however due to limited amount of tissue available, in 4 cases trabecular parameters could not be measured (indicated in brackets are the samples measured for trabecular parameters). 
were stored at room temperature until further use.

\section{Histological preparation and analysis}

Samples were decalcified using Luthra solution $(3.2 \% 11 \mathrm{M} \mathrm{HCl}, 10 \%$ formic acid in distilled water), dehydrated, cleared in xylene, embedded in paraffin and cut to yield $5 \mu \mathrm{m}$ sections. Sections were stained with fast green and Safranin-O for measurements of cartilage thickness (distance from the surface to the interface with the subchondral bone), and of the calcified cartilage zone (from tidemark to bone surface). Digital images were analyzed using Cell^ $F$ software (Olympus, USA). Average thickness of cartilage and calcified cartilage zone (CCZ) for each sample was determined by averaging 4 measurements per image taken from different locations of the section.

\section{Fourier-transform infrared imaging (FTIRI) and polarized light microscopy (PLM)}

Unstained histological sections were inserted in a controlled atmosphere chamber without humidity and specific regions of interest (ROIs) of the full thickness of the cartilaginous tissue (Fig. 1A) were measured using a Fourier transform infrared imaging system (PerkinElmer Spectrum Spotlight 300system). The absorption spectrum of a pixel $\left(25 \times 25 \mu^{2}\right)$ was translated to relative values (Fig. 1B). Collagen content was estimated with amide I (1585$1720 \mathrm{~cm}^{-1}$ ) absorption and PGs with absorption at carbohydrate region 984$1140 \mathrm{~cm}^{-1}[23,24]$ (Fig. 1C). The values obtained for each pixel within the ROI constituted a matrix with information on PG and collagen contents, which was subsequently used for detailed analysis of the zonal structure of cartilage tissue (Fig. 1B).

Collagen fiber orientation was visualized using PLM, and used for the classification of the superficial, middle and deep layer of cartilage [25, 30] (Fig. 2). An Abrio PLM system (Cri Inc., Woburn, MA, USA) mounted on a light microscope (Nikon Diaphot TMD, Nikon Inc.) was used for the PLM measurements. The area with the minimum birefringence value was assumed to be the border between the superficial and the middle zones, whereas the deep zone was considered to begin when the orientation angle values reached a plateau (typically close to 90 degrees with respect to cartilage surface) [31, 32] (Fig. 2B, E). Birefringence depends strongly on the anisotropy of collagen fibrils: birefringence is high when fibrils are aligned to same direction with 


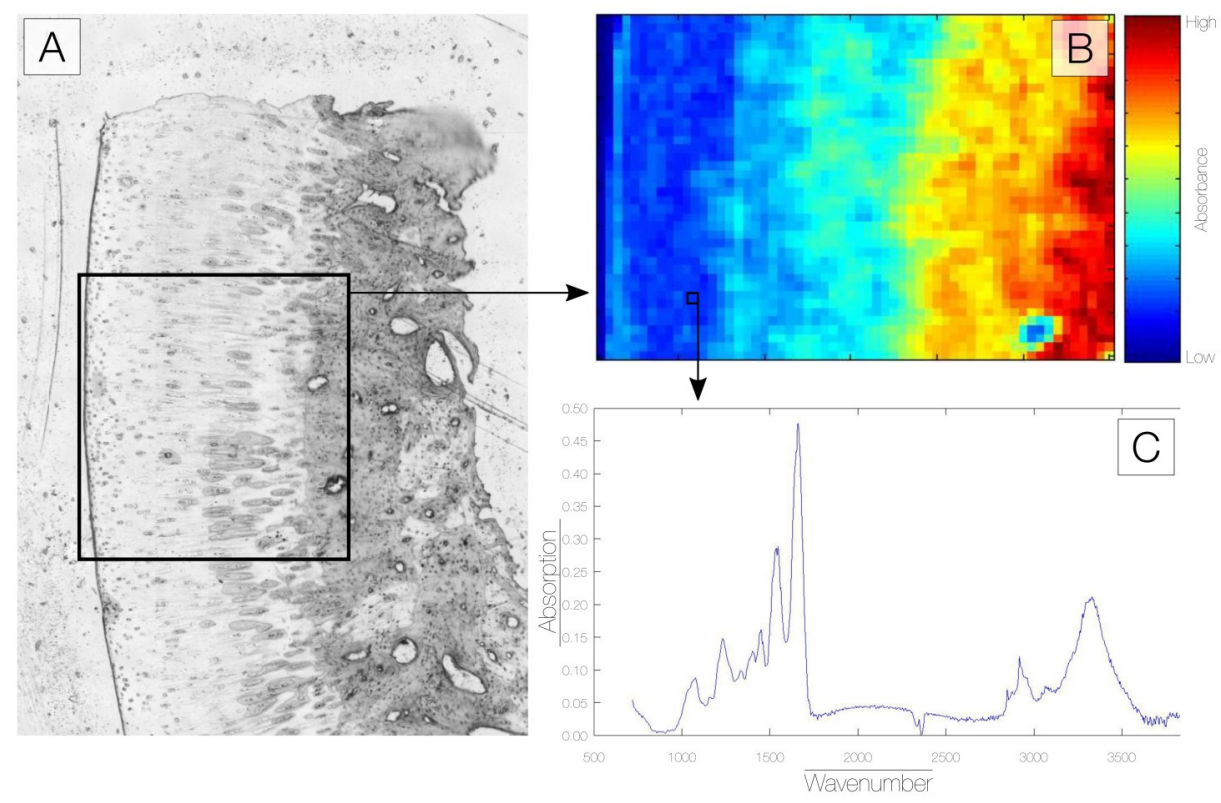

Figure 1. Fourier transform infrared imaging (FTIRI) methodology. ROIs were selected using as reference the margin of cartilage interfacing with the synovial joint space, and the interface with the subchondral bone plate (A). The ROI was divided in pixels of $25 \times 25 \mu \mathrm{m} 2$ (B, black square), and infrared absorption spectra were recorded for each pixel (C). The absorption for specific intervals was calculated to obtain relative contents of collagen and proteoglycans. Collagen content was estimated with amide I (1585-1720 $\mathrm{cm}^{-1}$ ) absorption and PGs with absorption at carbohydrate region (984-1140 $\left.\mathrm{cm}^{-1}\right)$. The values obtained for each pixel within the ROI constituted a matrix with information on PG and collagen contents, which was subsequently used for detailed analysis of the zonal structure of cartilage tissue.

each other, and, in contrast, low when fibrils are randomly oriented. Therefore, high birefringence is seen in superficial and deep zones of cartilage, but low birefringence is observed in the middle zone where the fibrils are randomly oriented. Based on this, the minimum birefringence value is assumed to represent the border between the superficial and middle zones [32] (Fig. 2C, F).

The combination of this information with the quantitative data contained in the matrix obtained with FTIRI allowed for relative quantification of proteoglycans and collagen in selected regions, and layer by layer. The PLM allowed also quantifying of individual cartilage layer thickness (superficial, middle and deep). 


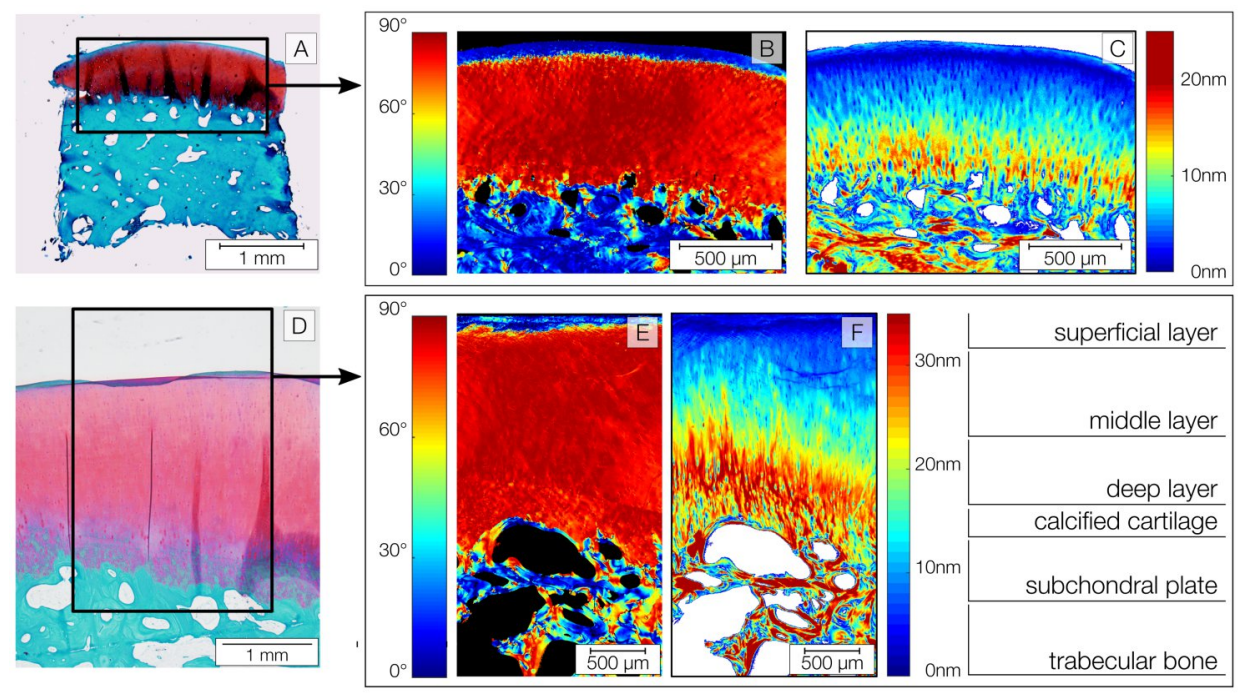

Figure 2. Osteochondral microscopic images of Thompson's gazelle (20 kg, Top) and Giraffe $(609.5 \mathrm{~kg}$, bottom). Left images show sections stained for glycosaminoglycans with safranin-O (A, C). Centre images are collagen orientation maps obtained using polarized light microscopy images $(P L M)(B, D)$. Blue colour indicates that fibers are parallel to the surface, while red indicates fibers having perpendicular orientation. The use of PLM allows determination of collagen fibers orientation, and consequently of the transition from superficial, to middle to deep layer of cartilage. Right images are retardance images obtained with PLM (birefrincence maps, C, F).

\section{Micro-CT}

Micro Computed Tomography (micro-CT) images of the osteochondral cores were obtained with a micro-CT scanner (Quantum FX, Perkin Elmer, USA, voxel size $=20 \mathrm{\mu m}^{3}$ ). The automatically reconstructed micro-CT images were subsequently converted to series of 2D TIFF images and were binarized using local thresholding (Bernsen technique). BoneJ software [33] was used to determine thickness and bone volume fraction (Sc.BV/TV) of the subchondral plate/calcified cartilage with manual selection of the ROI, which started from the beginning of mineralized tissue under cartilage, and continued to the beginning of trabecular bone (Fig. 3A); trabecular thickness (Tb.Th) and bone volume fraction (Tb.BV/TV) of the underlying trabecular bone were also determined with the same methodology. To obtain true subchondral plate thickness, CCZ thickness was measured by light microscopy (Fig. 3B), and its value was subtracted from the $\mathrm{CT}$ measurements to obtain real subchondral plate thickness (Sc.Th, Fig. 3C). 


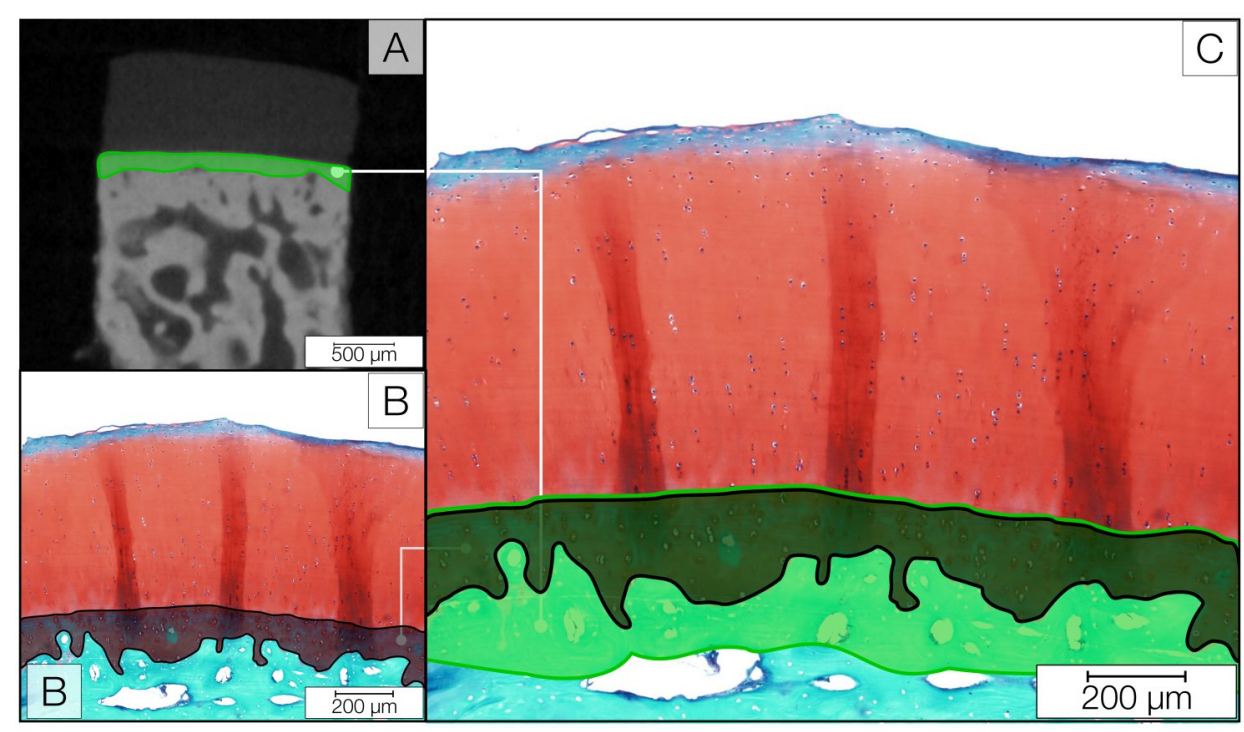

Figure 3. Micro-computed tomography (micro-CT) images of the osteochondral cores were obtained with a micro-CT scanner (Quantum FX, Perkin Elmer, USA, resolution 20 Mm). ROIs were selected manually (A, green), and used to determine subchondral plate thickness with BoneJ software. As micro-CT cannot discriminate between calcified cartilage and subchondral bone, CCZ thickness was measured using light microscopy ( $B$, black), and its value was subtracted from the CT measurements ( $C$, green selection) to obtain Sc Th. (For interpretation of the references to colour in this figure legend, the reader is referred to the web version of this article.)

\section{Statistics}

Initial statistical analysis was performed with R software 3.5.0 [34], to obtain a correlation matrix with Pearson coefficients [35, 36]. A weighted correlation of the parameters showing possible dependencies was performed using the Rpackage "wCorr" version 1.9.1. Comparisons between multiple groups were performed using a one-way ANOVA combined with post-hoc t-tests with Bonferroni correction. Statistical comparison of the obtained power coefficients with the theoretical coefficient of 0.33 (isometric scaling) was performed using a one-sample $t$-test. Limit of statistical significance was set at $p<0.05$. 


\section{RESULTS}

\section{Depth-wise architecture of articular cartilage}

Thickness of the cartilage layer (calcified plus non-calcified) varied widely between species, ranging from $64.6 \mu \mathrm{m}$ in the mouse to $3.25 \mathrm{~mm}$ in the African elephant. Calcified cartilage thickness ranged from $46.4 \mu \mathrm{m}$ in the mouse to $310 \mu \mathrm{m}$ in the African elephant. Total cartilage thickness correlated with body mass (BM) with a negative allometric relationship $\left(R^{2}=0.83, a=0.29\right.$, Fig. S1), in line with previous findings of Malda et al. [7].

Layer thickness of each layer was normalized to superficial layer thickness to show how layers relate to each other (Fig. 4A). Relative layer thickness was expressed for each layer as a percentage of total thickness, and correlated to $B M$. The relative thickness of the deep zone of the articular cartilage showed an increasing trend in relation to $\mathrm{BM}$, whereas the relative thicknesses of both the superficial and the middle zone showed a tendency to decrease

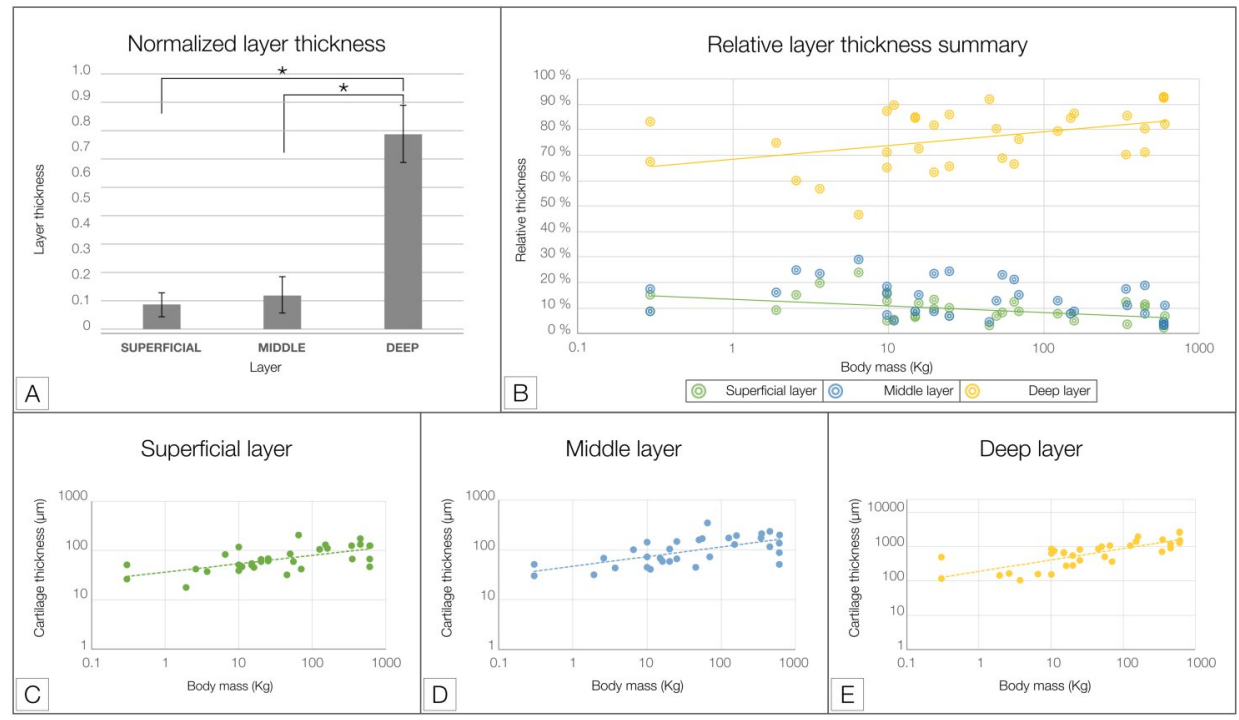

Figure 4. Summary of cartilage scaling along body mass. Thickness of each layer was normalized to total layer thickness to show layers' relation to each other (A), showing significant differences between layers ( ${ }^{*} p<0.05$ ). Relative layer thickness (\% of total thickness) was represented for each layer and correlated to BM (B). Relative deep zone thickness (yellow) showed an increasing trend in relation to $B M$ whereas relative superficial (green) and middle (blue) zone thickness showed a tendency to decrease along increasing $B M(B)$. Absolute values for the thickness of the single cartilage layers varied allometrically with $B M$ (superficial layer, $r=0.60, p<0,05, a=0.17, C$ ) (middle layer, $r=0.65, p<0.05, a=0.19, D)$ and isometrically with $B M$ for the deep layer $(r=0.82, p<0.05$, $a=0.33, E)$. 
proportionally in relation to body size (Fig. 4B). Absolute values for the thickness of the single cartilage layers varied allometrically with BM (superficial layer, $r=0.60, p<0.05, a=0.17$, Fig. 4C) (middle layer, $r=0.65, p<0.05$, $a=0.19$, Fig. $4 \mathrm{D})$ and isometrically with BM for the deep layer $(r=0.82, p<0.05$ $a=0.33$, Fig. 4E).

Combination of FTIRI and PLM techniques allowed for relative quantification and comparison (dimensionless numbers) of spatial collagen and PG content across species. Relative total collagen and PG content, when measured over the total thickness of the cartilage, showed no dependency to BM (Fig. 5A, B), with a small variation in composition across all species when considering content on the whole thickness of the tissue. Layer-by-layer analysis

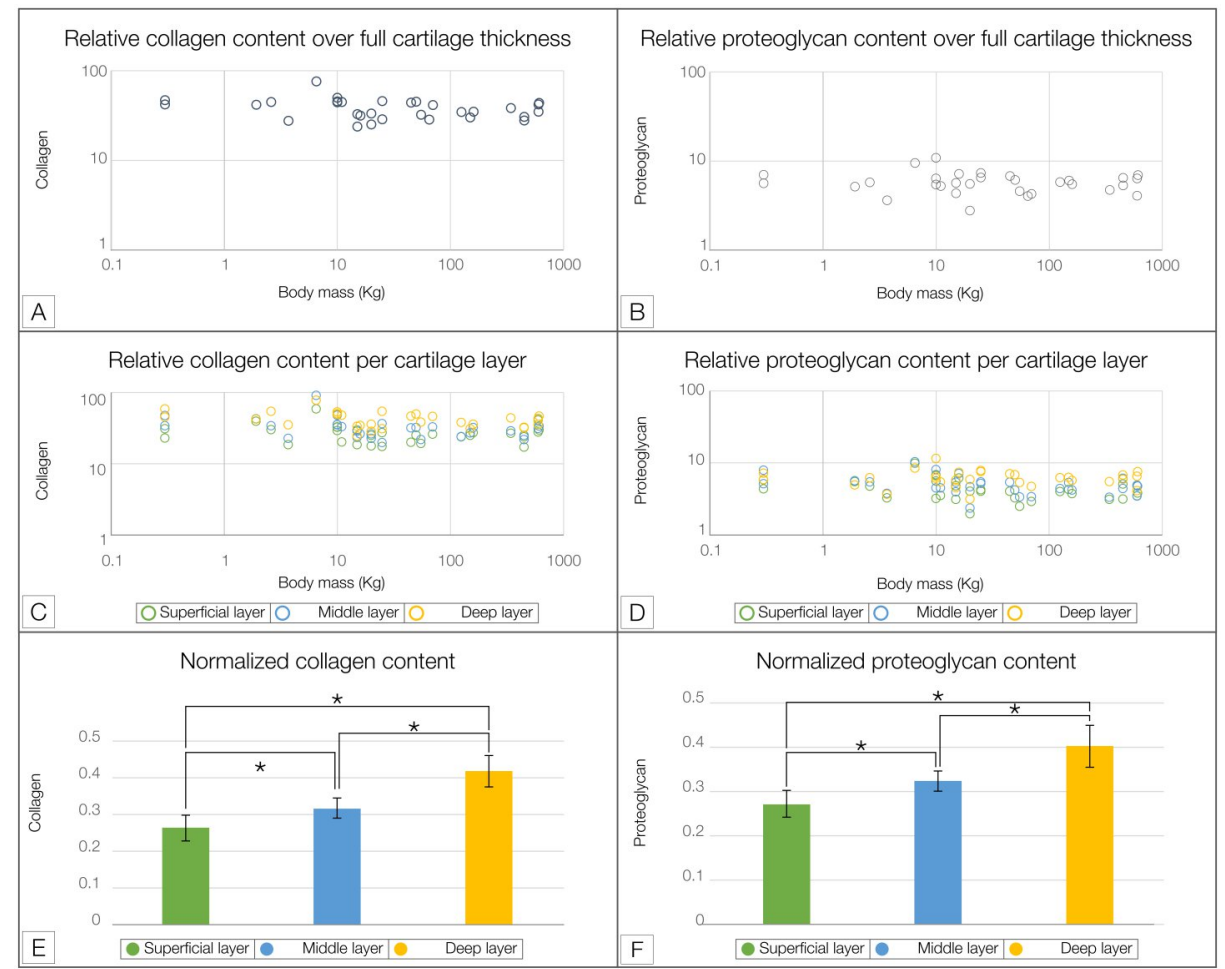

Figure 5. Relative collagen and proteoglycan contents in cartilage, measured over total thickness $(A, B$ respectively), and layer per layer $(C-D)$. Overall collagen and proteoglycan contents showed no dependency to body mass (collagen, B proteoglycans), with a small variation in cartilage composition across all species. Layer per layer analysis (superficial, middle and deep, respectively in green, blue and yellow) showed this was also true for both collagen and proteoglycans within each specific layer across species $(C, D)$. Normalizing the content of collagen and proteoglycans to total thickness content, deep layer content was highest, and superficial layer was lowest for both collagen and proteoglycans $(E, F)$, and showed significant differences between layers $\left({ }^{*} p<0.05\right)$. 

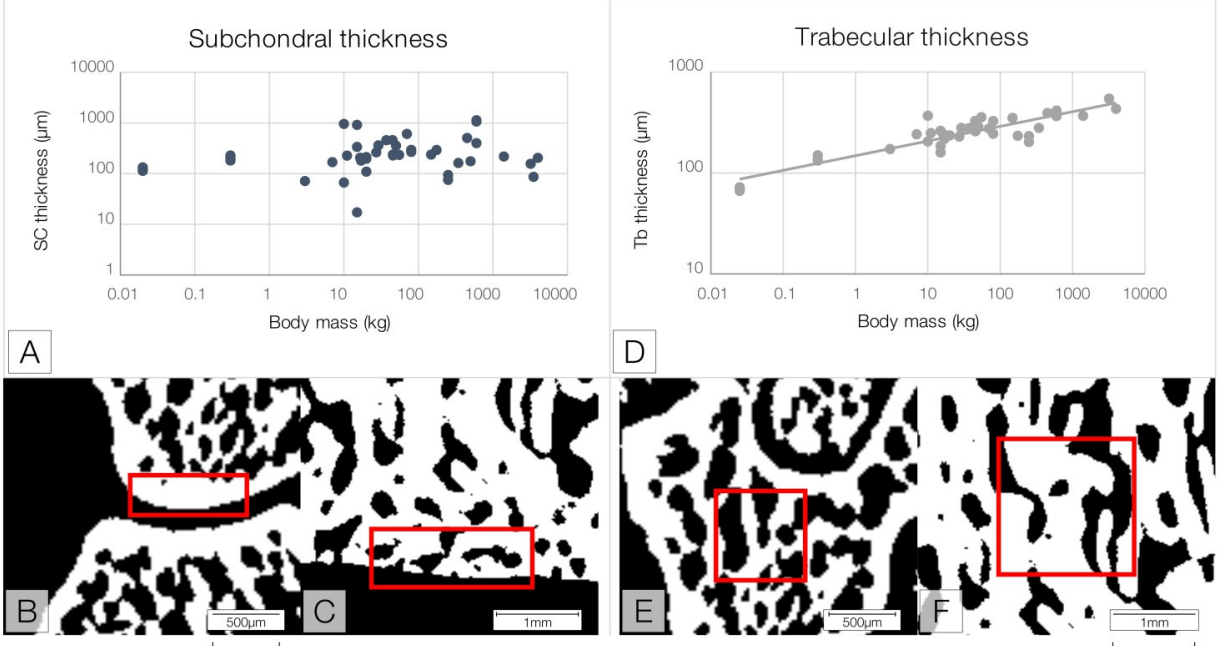

Figure 6. Scaling of subchondral plate and trabecular thickness. Subchondral plate thickness (Sc $T h$ ) showed no correlation to body mass (A). Images obtained with micro-CT of rat (B) and elephant (C) show small differences in subchondral plate (red square) thickness between animals of very different sizes. Trabecular thickness (Tb.Th) correlated to body mass (BM), with a negative allometric relationship to body mass $(r=0.72, a=0.14), D)$. Images obtained with micro-CT rat $(E)$ and elephant (F) show increase in trabecular bone (red square) thickness along BM.

(superficial, middle, deep) showed this was true in all cartilage layers for both collagen and proteoglycans (Fig. 5C, D). Normalizing the contents of collagen and PG to superficial layer content, deep layer content was highest, and superficial layer was lowest for both collagen and $P G$ and showed significant differences between layers (Fig. 5E, F, $p<0.05$ ).

\section{Subchondral and trabecular bone structure}

Subchondral bone volume fraction (Sc.BV/TV) showed a positive correlation to Sc.Th $(r=0.75, p<0.001)$. No correlation was found between Sc.BV/TV and Sc.Th to BM (Fig.6A). The thickness of the subchondral plate ranged from $67.25 \mu \mathrm{m}$ in the mouse to $1.13 \mathrm{~mm}$ in the horse. Mean subchondral plate thickness (Sc.Th, Table S1) was highest in the equine species (901 $\pm 344 \mu \mathrm{m}$, $n=3$ ), while in the rats it was $205 \pm 24 \mu \mathrm{m}(n=3)$ (Fig. 6B), and in the elephants the thickness was $149 \pm 59 \mu \mathrm{m}(n=3)$ (Fig. 6C). Sc.BV/TV ranged from $95.8 \% \pm$ $1.86 \%$ in mice $(n=3)$ to $99.7 \% \pm 0.15 \%$ in horses $(n=3)$.

Trabecular thickness (Tb.Th) correlated with $\mathrm{BM}$ in a negative allometric relationship ( $r=0.72, p<0.001, a=0.14$, Fig. 6D). Trabecular thickness (Tb.Th, Table S1) ranged from $69.22 \pm 2.87 \mu \mathrm{m}(n=3)$ in the mouse, $139.6 \pm 9.13 \mu \mathrm{m}$ 
$(n=3)$ in the rats (Fig. 6E), to $489.37 \pm 79.86 \mu \mathrm{m}(n=2)$ in the elephants (Fig. 6F). Trabecular bone volume fraction (Tb.BV/TV) showed a positive correlation with $\mathrm{BM}(r=0.36, p<0.05)$, and trabecular thickness and trabecular BV/TV also showed a strong correlation between each other $(r=0.67, p<0.001)$.

An analysis of the log ratio of thickness of cartilage, subchondral bone and trabecular bone, was performed to visualize differences between animals at the extremities of our BM range (i.e. belonging to the Muridae family and to the Elephantidae family), however statistical analysis revealed no significant differences (Fig. 7).

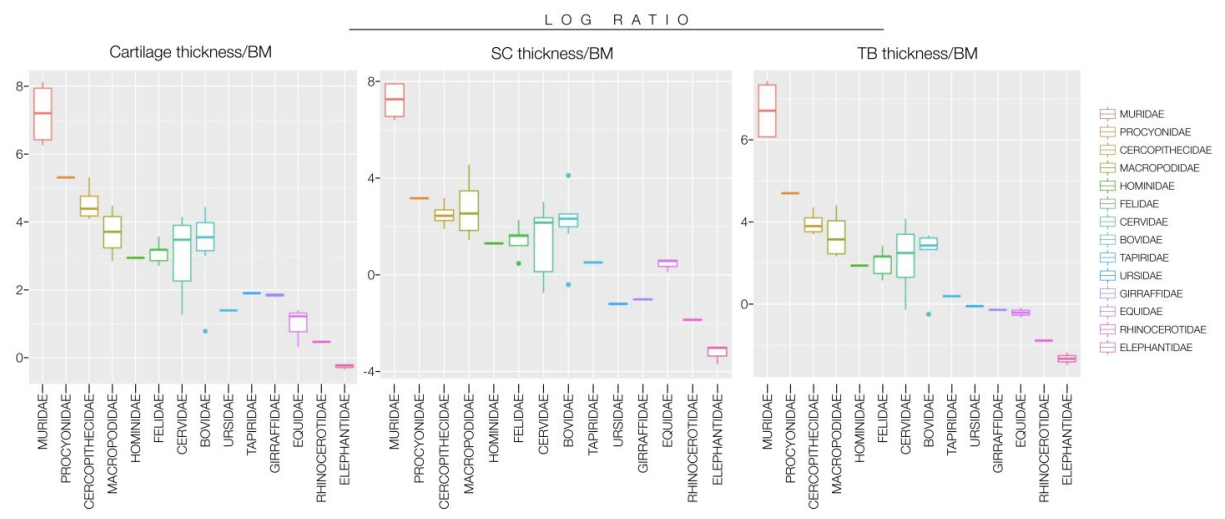

Figure 7. Analysis of the log ratios of cartilage, subchondral plate and trabecular bone thicknesses, was performed to visualize possible differences between animals at the extremities of BM range (i.e. belonging to the Muridae family and to the Elephantidae family), however statistical analysis could not highlight any significant differences.

\section{DISCUSSION}

A comprehensive analysis of how the osteochondral unit changes with body mass was performed using a combination of different techniques for measuring the microstructural features. Overall articular cartilage thickness scaled in a negative allometric fashion, confirming earlier findings [7].

The current study showed that, contrary to our first hypothesis, both superficial and intermediate layers become relatively thinner single layer, whereas the deep layer seems to scale isometrically. The explanation may be that the deep zone is richest in PGs and hence thought to be primary responsible of transmission of load to the underlying bone [37-39], and therefore may need to scale accordingly to BM. This would also be in line with the role that is 
attributed to the superficial layer [40,41], which is thought to have a major role in the homogeneous distribution of the impact forces and loads away from directly-loaded regions $[42,43]$, more than in load attenuation and with the fact that in physics, as one scales down, forces like viscous drag become more important than weight $[1,44]$.

The biochemical composition of articular cartilage was remarkably consistent across species in both absolute and relative terms. This suggests that evolutionary pressure has led to the best possible combination of PG and collagen to effectuate the duty of shock-absorption and transfer of forces to the subchondral and trabecular bone in terrestrial locomotion. The normalization of content to total layer thickness confirmed layer dependency highlighting significant difference between the deep layer and the superficial and middle layers $\left({ }^{*} p<0.05\right)$, as was expected from previous studies on selected species [45]. However, as remarkable as it seems that there are no substantial variations in the major structural components of cartilage from mouse to elephant, there may be some in characteristics of those components that were not specifically measured, such as the post-translational modifications of collagen of which cross-links are the most likely candidates.

Benninghoff (1925) first described the arching structure formed by the collagen fibers of articular cartilage that run from their anchoring site in the calcified zone first through the deep zone, directed perpendicularly to the subchondral plate, to then describe an arch at the beginning of the transitional zone, with the keystone of the arch in or near the superficial zone where the fiber runs tangential to the cartilage surface before starting its return journey back to the subchondral bone, forming the second pillar of the arch [46]. If we assume the arching parts to be semi-circular, the thickness of the middle zone is theoretically given by the radius of the arches. The constant thickness of the superficial and middle layers and the increasing thickness of the deep layer with increasing total cartilage thickness suggest that the radius of the arches remains constant with their pillars becoming longer. That would mean that the adaptation of the collagen architecture to scaling would consist of the arches becoming more slender with increasing cartilage thickness; and not proportionally increase in size. In this case, the relative number of arches per unit of (subchondral bone plate) surface would remain constant and, given the constant ratio of total collagen to total mass of cartilage, the ratio of collagen fibril thickness to total cartilage thickness would not increase isometrically with the 
pillars of the arches becoming relatively thinner. However, verification of this theory would require large numbers of samples from differently sized animals from the same species, as there are relatively large differences in configuration of the collagen arches over the species [15].

Interestingly, the response of the subchondral unit seems to be independent of increasing body mass. Although it was hypothesized that the subchondral bone plate thickness would scale with body mass as well, this was not the case. The subchondral bone volume fraction (and subsequently porosity) showed no correlation with BM. This is unexpected, because if the porosity of the interface between cartilage and bone increases in larger animals, this could be explained by the need of a less dense structure that could be more able to effectively distribute and transmit forces homogeneously to the underlying trabecular bone [47].

The trabecular bone itself features absolute thicker trabeculae and is denser with increasing body mass to accommodate the higher forces. This could permit accommodation of higher forces and be interpreted as an adaptation to increased body mass. A relatively less dense structure could also allow better nutrition efficiency towards the cartilage by facilitating diffusion from the subchondral bone. However, this should be confirmed by an analysis of microand nano- porosity, which would require imaging with a higher resolution than possible with light microscopy. There is in fact evidence in literature that, in an experimental setting, there is exchange of nutrients at the interface of bone and cartilage, although of minimal order compared to the nutrient exchange with the synovial fluid [48]. In fact, Arkill et al. 2008, reported that areas of direct contact of non-calcified cartilage with the subchondral bone allow for a five-fold solute exchange compared to calcified regions [49]. Furthermore they showed that even calcified cartilage is permeable to small solutes so that the subchondral circulation may indeed have a significant role in nutrition of the deep cartilage layer [49]. However, the absence of this correlation suggests that more complex factors may be at play: a possible determinant could reside in the mutable nature of the interface, which may be more dynamic than initially thought [50]. There is abundant evidence suggesting that bone and cartilage crosstalk, as shown per example by the presence of vascular canals in the interface between subchondral bone and calcified cartilage [51, 52]. These characteristics suggest that the interface may be less directly influenced by biomechanical loading, as more complex influences contribute to its micro- 
structural features.

The scaling of the trabecular bone features confirmed our hypothesis of negative allometry, with a slope value $(a=0.14)$ in line with previous studies [9]. Interestingly, this relationship was shown to change to isometry when comparing only primates, as demonstrated by a meta-analysis conducted on over 30 primate species by Ryan et al. [53]. The analysis of the relative thickness of cartilage, subchondral plate and trabeculae (Fig. 7) suggests that size may impose different rules at the extremities of the weight spectrum. The relationship seems to be rather similar amongst most species and sizes, but very small and very large animals appear to have their own ratios when it comes to scaling. Some of these exceptions have been reported in literature, mostly with respect to the smaller species such as mice and rats in which trabecular bone features [9] and cartilage cellularity [7] were shown not to follow the general patterns. Data on the other end of the spectrum are virtually lacking. A much higher sample pool would be needed for statistical confirmation of these trends.

While offering new insights into the variation in the osteochondral unit structure across species, this study has some limitations. Traditional biochemistry still remains the gold standard for the characterization of extracellular matrix composition, but was replaced by FTIRI analysis in this study. This was done because traditional biochemical analysis of the different cartilage layers would have been very difficult and subject to various sources of error [24]. The use of FTIRI has previously been applied for spatial analysis of the main ECM components of cartilage (i.e. collagen and proteoglycans) [21-23], but is performed under controlled atmospheric conditions, in absence of water and on dehydrated sections [21]. This may affect thickness measurements due to shrinkage of samples related to fixation for paraffin embedding; however, it should be noted that the order of shrinkage is largely dependent on water content [54], and is common to all morphological studies performed using fixated tissue. Further, the distribution of components should remain relatively unaltered [55] and in the current study the overall biochemical composition of cartilage was found to be similar to earlier reports using classic biochemical analysis methods [7].

Lastly, standard osteochondral units of $6 \mathrm{~mm}$ diameter were harvested and analyzed in all species. These were taken from a weight bearing area of the medial condyle of the femur. In the mouse this means that the sample con- 
sisted of virtually the entire condyle, while in the elephant it represented a small portion on the articular surface of the medial condyle. Since it is known that there is topographical heterogeneity in cartilage composition related to weight-bearing $[38,56]$, this means that in the larger species variability may be a little larger depending on exact sample location. However, this effect was most likely limited, as samples were in all cases taken from a load-bearing area. Finally, because samples were harvested from deceased animals brought for necroscopy at Utrecht University, sample size was very limited for some species.

\section{CONCLUSIONS}

Articular cartilage is a tissue with a high degree of specialization that, in a functional sense, can only be appreciated in the wider context of the osteochondral unit. Our findings suggest that the tissue's structure has remained remarkably preserved across mammalian species during evolution, and that the trabecular and subchondral bone -in particular- adapt to the increasing body mass. The natural constancy and apparent immutability of the cartilage should be considered when designing strategies for regeneration and/or functional repair.

\section{ACKNOWLEDGEMENTS}

The authors would like to thank: Chris Van de Lest for the statistical consultancy, Virpi Tiitu for the PLM contribution, Miguel Castilho for the discussion, and VariaVision -Science Division for the figures consultancy. The research leading to these results has received funding from the European Community's Seventh Framework Programme (FP7/2007- 2013) under grant agreement 309962 (HydroZONES), the European Research Council under grant agreement 647426 (3D-JOINT), and the Dutch Arthritis Foundation (LLP-12 and LLP-22). 


\section{REFERENCES}

[1] Galilei G. Discorsi e dimostrazioni matematiche intorno a due nuove scienze attenenti alla meccanica e i movimenti locali. Leiden, The Netherlands: Ludovico Elzeviri; 1638.

[2] Allen DH. How mechanics shaped the modern world: Springer; 2014.

[3] Biewener AA. Scaling body support in mammals: limb posture and muscle mechanics. Science. 1989;245:45-8.

[4] Carrano MT. Implications of limb bone scaling, curvature and eccentricity in mammals and non-avian dinosaurs. Journal of Zoology. 2001;254:41-55.

[5] Simon WH. Scale effects in animal joints. I. Articular cartilage thickness and compressive stress. Arthritis \& Rheumatism: Official Journal of the American College of Rheumatology. 1970;13:244-55.

[6] Biewener AA. Biomechanical consequences of scaling. Journal of Experimental Biology. 2005;208:1665-76.

[7] Malda J, de Grauw JC, Benders KE, Kik MJ, van de Lest CH, Creemers LB, et al. Of mice, men and elephants: the relation between articular cartilage thickness and body mass. PLoS One. 2013;8:e57683.

[8] Doube M, Kłosowski MM, Wiktorowicz-Conroy AM, Hutchinson JR, Shefelbine SJ. Trabecular bone scales allometrically in mammals and birds. Proceedings of the Royal Society of London B: Biological Sciences. 2011:rspb20110069.

[9] Barak MM, Lieberman DE, Hublin J-J. Of mice, rats and men: trabecular bone architecture in mammals scales to body mass with negative allometry. Journal of structural biology. 2013;183:123-31.

[10] Chevrier A, Kouao AS, Picard G, Hurtig MB, Buschmann MD. Interspecies comparison of subchondral bone properties important for cartilage repair. J Orthop Res. 2015;33:63-70.

[11] Lories RJ, Luyten FP. The bone-cartilage unit in osteoarthritis. Nature Reviews Rheumatology. 2011;7:43.

[12] Kawcak CE, Mcllwraith CW, Norrdin R, Park R, James S. The role of subchondral bone in joint disease: a review. Equine veterinary journal. 2001;33:120-6.

[13] Hunziker E. Articular cartilage repair: basic science and clinical progress. A review of the current status and prospects. Osteoarthritis and cartilage. 2002;10:432-63.

[14] Guilak F. Biomechanical factors in osteoarthritis. Best practice \& research Clinical rheumatology. 2011;25:815-23.

[15] Kääb M, Ap Gwynn I, Nötzli H. Collagen fibre arrangement in the tibial plateau articular cartilage of man and other mammalian species. The Journal of Anatomy. 1998;193:23-34.

[16] Frisbie D, Cross M, Mcllwraith C. A comparative study of articular cartilage thickness in the stifle of animal species used in human pre-clinical studies compared to articular cartilage thickness in the human knee. Veterinary and comparative orthopaedics and traumatology. 2006;19:142-6.

[17] Jürgens KD, Bartels $H$, Bartels R. Blood oxygen transport and organ weights of small bats and small non-flying mammals. Respiration physiology. 1981;45:243-60.

[18] Froese R. Cube law, condition factor and weight-length relationships: history, meta-analysis and recommendations. Journal of applied ichthyology. 2006;22:241-53.

[19] Klein TJ, Malda J, Sah RL, Hutmacher DW. Tissue engineering of articular cartilage with biomimetic zones. Tissue Engineering Part B: Reviews. 2009;15:143-57.

[20] Currey J. The many adaptations of bone. Journal of biomechanics. 2003;36:1487-95.

[21] Camacho NP, West P, Torzilli PA, Mendelsohn R. FTIR microscopic imaging of collagen and proteoglycan in bovine cartilage. Biopolymers. 2001;62:1-8.

[22] Boskey A, Camacho NP. FT-IR imaging of native and tissue-engineered bone and cartilage. Biomaterials. 2007;28:2465-78.

[23] Potter K, Kidder LH, Levin IW, Lewis EN, Spencer RG. Imaging of collagen and proteoglycan in cartilage sections using Fourier transform infrared spectral imaging. Arthritis \& Rheumatology. 2001;44:846-55. 
[24] Rieppo L, Saarakkala S, Närhi T, Holopainen J, Lammi M, Helminen H, et al. Quantitative analysis of spatial proteoglycan content in articular cartilage with Fourier transform infrared imaging spectroscopy: critical evaluation of analysis methods and specificity of the parameters. Microscopy Research and Technique. 2010;73:503-12.

[25] Changoor A, Tran-Khanh N, Methot S, Garon M, Hurtig M, Shive M, et al. A polarized light microscopy method for accurate and reliable grading of collagen organization in cartilage repair. Osteoarthritis and cartilage. 2011;19:126-35.

[26] Yarker Y, Aspden R, Hukins D. Birefringence of articular cartilage and the distribution of collagen fibril orientations. Connective tissue research. 1983;11:207-13.

[27] Speer DP, Dahners L. The collagenous architecture of articular cartilage. Correlation of scanning electron microscopy and polarized light microscopy observations. Clinical orthopaedics and related research. 1979:267-75.

[28] Fajardo R, Müller R. Three-dimensional analysis of nonhuman primate trabecular architecture using micro-computed tomography. American journal of physical anthropology. 2001;115:327-36.

[29] Holdsworth DW, Thornton MM. Micro-CT in small animal and specimen imaging. Trends in Biotechnology. 2002;20:S34-S9.

[30] Rieppo J, Hallikainen J, Jurvelin JS, Kiviranta I, Helminen HJ, Hyttinen MM. Practical considerations in the use of polarized light microscopy in the analysis of the collagen network in articular cartilage. Microscopy research and technique. 2008;71:279-87.

[31] Julkunen P, Kiviranta P, Wilson W, Jurvelin JS, Korhonen RK. Characterization of articular cartilage by combining microscopic analysis with a fibril-reinforced finite-element model. Journal of biomechanics. 2007;40:1862-70.

[32] Arokoski J, Hyttinen MM, Lapveteläinen T, Takács P, Kosztáczky B, Módis L, et al. Decreased birefringence of the superficial zone collagen network in the canine knee (stifle) articular cartilage after long distance running training, detected by quantitative polarised light microscopy. Annals of the rheumatic diseases. 1996;55:253.

[33] Doube M, Kłosowski MM, Arganda-Carreras I, Cordelières FP, Dougherty RP, Jackson JS, et al. BoneJ: free and extensible bone image analysis in ImageJ. Bone. 2010;47:1076-9.

[34] Team RC. R: A language and environment for statistical computing

. Vienna, Austria.: R Foundation for Statistical Computing; 2018.

[35] Brian G. Peterson PC. Performance Analytics: Econometric Tools for Performance and Risk Analysis. R package version 1.5.2 ed2018.

[36] Wickham H. ggplot2: Elegant Graphics for Data Analysis. New York: Springer-Verlag; 2016.

[37] Mow VC, Ratcliffe A, Poole AR. Cartilage and diarthrodial joints as paradigms for hierarchical materials and structures. Biomaterials. 1992;13:67-97.

[38] Brama P, Tekoppele J, Bank R, Karssenberg D, Barneveld A, Weeren P. Topographical mapping of biochemical properties of articular cartilage in the equine fetlock joint. Equine veterinary journal. 2000;32:19-26.

[39] Kumar P, Oka M, Toguchida J, Kobayashi M, Uchida E, Nakamura T, et al. Role of uppermost superficial surface layer of articular cartilage in the lubrication mechanism of joints. The Journal of Anatomy. 2001;199:241-50.

[40] Sophia Fox AJ, Bedi A, Rodeo SA. The basic science of articular cartilage: structure, composition, and function. Sports health. 2009;1:461-8.

[41] Muir H, Bullough P, Maroudas A. The distribution of collagen in human articular cartilage with some of its physiological implications. The Journal of bone and joint surgery British volume. 1970;52:554-63.

[42] Bevill S, Thambyah A, Broom N. New insights into the role of the superficial tangential zone in influencing the microstructural response of articular cartilage to compression. Osteoarthritis and Cartilage. 2010;18:1310-8.

[43] Korhonen R, Wong M, Arokoski J, Lindgren R, Helminen H, Hunziker E, et al. Importance of the superficial tissue layer for the indentation stiffness of articular cartilage. Medical Engineering and Physics. 2002;24:99-108.

[44] Peterson MA. Galileo's discovery of scaling laws. American Journal of Physics. 2002;70:575- 
80.

[45] Malda J, Benders K, Klein T, De Grauw J, Kik M, Hutmacher D, et al. Comparative study of depth-dependent characteristics of equine and human osteochondral tissue from the medial and lateral femoral condyles. Osteoarthritis Cartilage. 2012;20:1147-51.

[46] Benninghoff A. Form und Bau der Gelenkknorpel in ihren Beziehungen zur Funktion. Zeitschrift für Zellforschung und mikroskopische Anatomie. 1925;2:783-862.

[47] Pałka K, Pokrowiecki R. Porous Titanium Implants: A Review. Advanced Engineering Materials. 2018.

[48] Pouran B, Arbabi V, Bleys RL, van Weeren PR, Zadpoor AA, Weinans H. Solute transport at the interface of cartilage and subchondral bone plate: effect of micro-architecture. Journal of biomechanics. 2017;52:148-54.

[49] Arkill K, Winlove C. Solute transport in the deep and calcified zones of articular cartilage. Osteoarthritis and Cartilage. 2008;16:708-14.

[50] Findlay DM, Kuliwaba JS. Bone-cartilage crosstalk: a conversation for understanding osteoarthritis. Bone research. 2016;4:16028.

[51] Clark J, Huber J. The structure of the human subchondral plate. The Journal of bone and joint surgery British volume. 1990;72:866-73.

[52] Imhof H, Sulzbacher I, Grampp S, Czerny C, Youssefzadeh S, Kainberger F. Subchondral bone and cartilage disease: a rediscovered functional unit. Investigative radiology. 2000;35:581-8.

[53] Ryan TM, Shaw CN. Trabecular bone microstructure scales allometrically in the primate humerus and femur. Proceedings of the Royal Society of London B: Biological Sciences. 2013;280:20130172.

[54] Hunziker EB, Lippuner K, Shintani N. How best to preserve and reveal the structural intricacies of cartilaginous tissue. Matrix biology. 2014;39:33-43.

[55] Kiviranta J, Tammi M, Jurvelin J, Säämänen A-M, Helminen H. Fixation, decalcification, and tissue processing effects on articular cartilage proteoglycans. Histochemistry. 1984;80:569-73.

[56] Kiviranta I, Jurvelin J, Tammi M, SääMäunen AM, Helminen HJ. Weight bearing controls glycosaminoglycan concentration and articualr cartilage thickness in the knee joints of young beagle dogs. Arthritis \& Rheumatology. 1987;30:801-9. 


\section{SUPPLEMENTAL DATA}

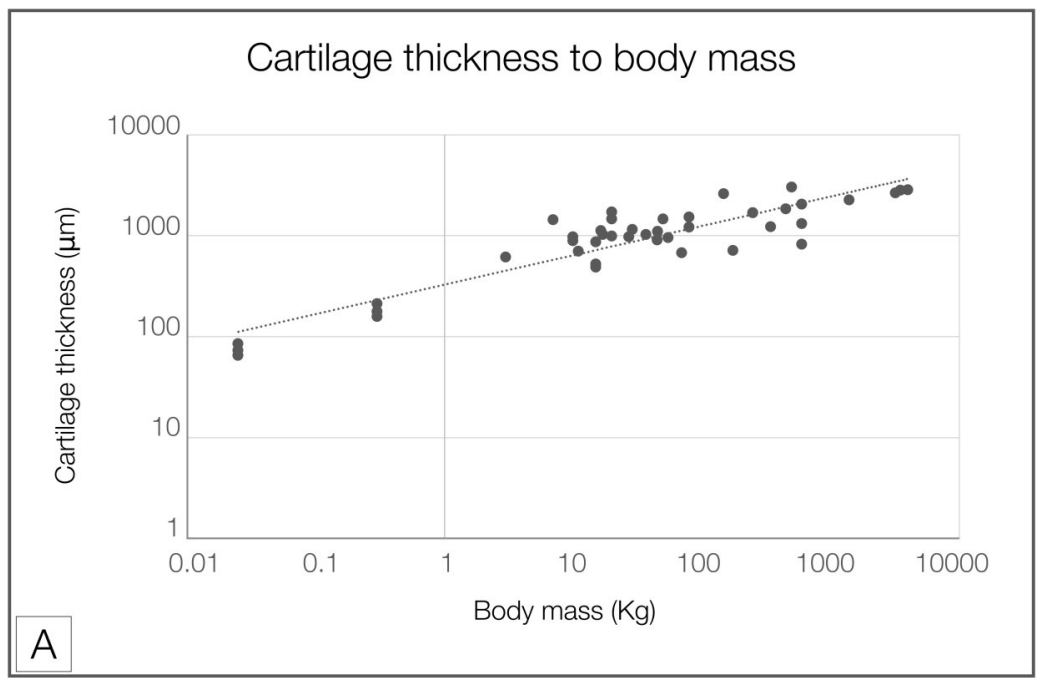

Figure S1. Total cartilage thickness correlated with body mass (BM) with a negative allometric relationship $\left(R^{2}=0.83, a=0.29\right)$, in line with previous findings of Malda et al. [3] 


\begin{tabular}{lrr}
\hline Species & Subchondral plate thickness $(\mu \mathrm{m})$ & Trabecular thickness $(\mu \mathrm{m})$ \\
\hline Mouse (Mus musculus) & 68.83 & 69.22 \\
Rat (Rattus sp) & 204.82 & 139.60 \\
South American coati (Nasua nasua) & 71.45 & 172.98 \\
Barbary macaque (Macaca sylvanus) & 117.72 & 224.03 \\
Tammar wallaby (Macropus eugenii) & 647.57 & 316.08 \\
Hamadryas baboon (Papio hamadryas) & 193.48 & 229.23 \\
Roe deer (Capreolus capreolus) & 152.13 & 204.37 \\
Thomson's gazelle (Eudorcas thomsonii) & 408.00 & 210.87 \\
Dwarf goat (Capra aegragus hircus) & 361.93 & 280.36 \\
Cheetah (Acinonyx jubatus) & 243.56 & 249.67 \\
Impala (Aepyceros melampus) & 458.46 & 303.44 \\
Red kangaroo (Macropus rufus) & 295.81 & 322.29 \\
Fallow deer (Dama dama) & 608.97 & 276.48 \\
Gorilla (Gorilla gorilla) & 294.47 & 327.76 \\
Siberian tiger (Panthera tigris) & 268.91 & 245.18 \\
Lion (Panthera leo) & 237.72 & 352.46 \\
Polar bear (Ursus maritimus) & 292.83 & 234.20 \\
South American tapir (Tapirus terrestris) & 75.35 & 232.20 \\
Elk (Alces alces) & 162.46 & 279.92 \\
Rothschild's giraffe (Giraffa camelopardalis) & 134.89 & 203.12 \\
Horse (Equus ferus caballus) & 901.74 & 380.42 \\
Banteng (Bos javanicus) & 400.03 & 415.78 \\
White rhinoceros (Ceratotherium simum) & 369.36 \\
Asian elephant (Elephas maximus) & 545.84 \\
African elephant (Loxodonta africana) & 432.90 \\
\hline
\end{tabular}

Table S1. Average subchondral plate thickness (Sc.Th, middle column) and trabecular thickness (Tb.Th, right column) in different species. 


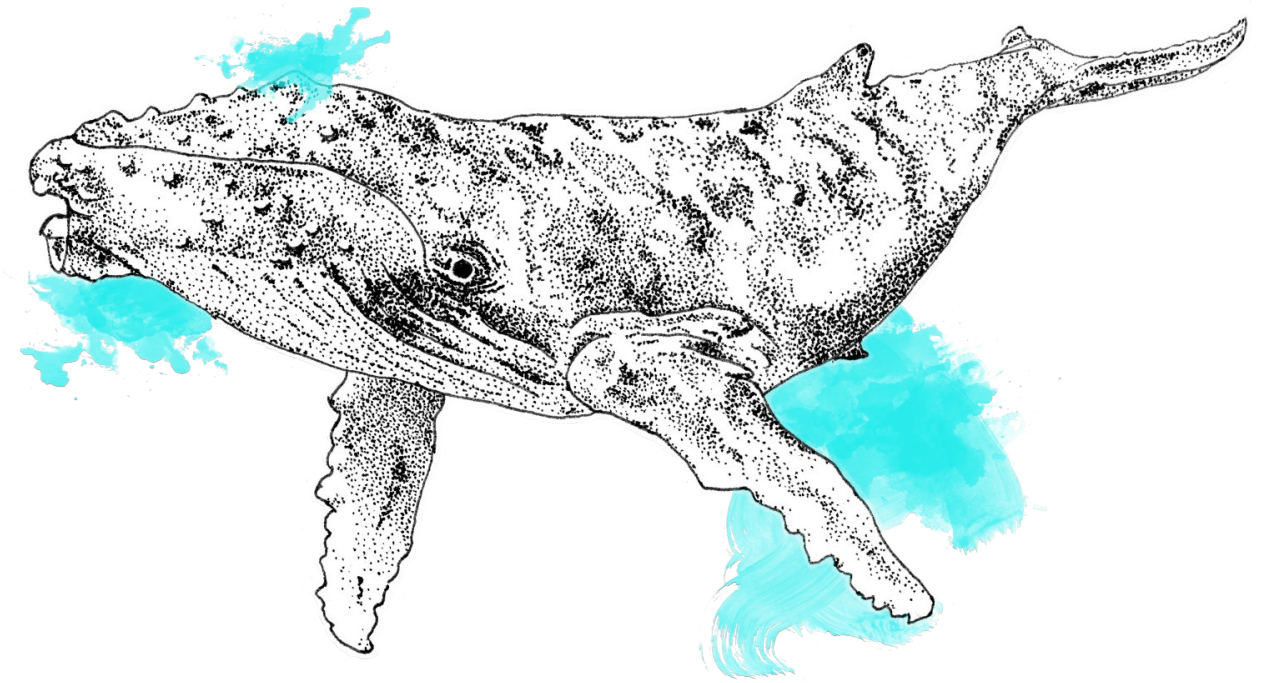




\title{
Chapter
}

Nature's design of the osteochondral unit: microstructural differences between terrestrial and aquatic mammals

\author{
I.A.D. Mancini \\ R. Levato \\ M. Castilho \\ M. Chen \\ M.H.P. van Rijen \\ M. Kik \\ J. Malda \\ P.R. van Weeren
}

A manuscript based on this chapter has been submitted 


\section{ABSTRACT}

During evolution, animals have returned from land to water, adopting morphological modifications to life in an aquatic environment. We compared the osteochondral units of marine and terrestrial mammals across species spanning a wide range of body weights, focusing on microstructural organization and biomechanical performance, which are pivotal elements for healthy locomotion. Aquatic mammals display cartilage with essentially random collagen fiber configuration, lacking the depth-dependent, arcade-like organization characteristic of terrestrial species. At the osteochondral interface, differently from terrestrial mammals, aquatic mammals lack a calcified cartilage layer and display a thin, highly porous subchondral bone plate. In terrestrial mammals cartilage was stiffer at equilibrium and showed a significantly higher peak modulus than in aquatic mammals. These differences in the osteochondral unit reflect the primordial importance of coping with loading for proper joint function. Recognizing such microarchitecture-function relationship is crucial for understanding articular biology and for developing regenerative approaches for joint damage. 


\section{INTRODUCTION}

In the course of evolution, mammals have gone from land to water at several occasions, resulting in separate lineages ranging from Cetacea (which include animals such as whales and dolphins) to diverging lines, such as the polar bear (Ursus maritimus) and sea otter (Enhydra lutris) [1]. Polar bears and sea otters have maintained their terrestrial form despite spending a significant portion of their life in water, while other species, in their evolutionary process, have become permanent residents of the aquatic environment and undergone more considerable morphological adaptations to marine life [1]. This long adaptation process that started in the Eocene, is particularly evident in Cetaceans [1, 2], where thoracic limbs became flippers, hind limbs regressed and disappeared, and vertebral bones adopted a more uniform morphology [3, 4].

The main drive for these changes was obviously meeting the physical demands of life in an aquatic environment, where locomotion is radically different. In water, buoyancy changes the influence of gravity dramatically and water is also denser and more viscous than air, influencing resistance [5]. Therefore, in aquatic mammals, structures originally specialized for movement on land became exposed to a completely different type of locomotion without weight-bearing [6]. Locomotion in water happens when the mechanism that propels the animal forward is efficient and powerful enough to counter the effects of drag. Different mammalian species have found specialized solutions to this problem in the form of diverse morphologies of their body and limbs (or fins), however always with the common denominator of the development of propulsive surfaces that oscillate to generate thrust. Swimming of aquatic mammals differs significantly in mechanical efficiency and stress distribution from locomotion in terrestrial mammals relying on limb movement. Pinnipeds, Cetaceans and Sirenians descend from mammals that evolved to move limbs in a vertical plane, therefore they use a hydrodynamic lift-based momentum exchange to move through water, using strokes parallel to the sagittal plane of the body when they swim [7].

In all mammals, regardless of if they live on land or in water, locomotion is effectuated by the musculoskeletal system. Its framework is composed by the skeleton, whose bony components articulate via joints. The function of synovial joints in particular, is to minimize frictions between those bony 
components by providing lubrication and to accommodate the biomechanical forces generated by locomotion, transmitting and dampening these forces [8]. The fundamental element that enables physiological function of the synovial joint is the osteochondral unit, composed of articular cartilage and bone. In the front extremities, synovial joints are the principal joint type in terrestrial mammals; in aquatic mammals they have been preserved during evolution in the humerus, radius and ulna [9], but have been replaced by fibrous joints in the distal joints of flippers, where bony elements are connected by dense connective tissue [10].

Comparative studies on the musculoskeletal system of terrestrial and aquatic mammals have largely focused on bone differences. This is probably because bone is a tissue known to respond extensively and quickly to changes in loading as described by Wolff's law and Frost's mechanostat theory [18, 19]. Studies on the macroscopic structures have shown that the bones of the Cetacean arm and forearm adopted an hourglass-like form, and the medullary cavity disappeared to strengthen flipper resistance for steering during fast swimming [11, 12]. At tissue level, adaptations of the bone have been described for both terrestrial [13, 14] and aquatic mammals [15], with a particular focus on how aquatic mammals manage buoyancy by variations in their structural bone density $[15,16]$. However, there is only limited understanding of the architecture of the cartilage extracellular matrix and of the bone microstructure, as well as of their impact on the mechanical properties of the whole osteochondral unit .

The osteochondral unit is the pivotal element of the joint, with a fundamental clinical relevance in joint disorders and damage as, for example, in osteoarthritis (OA), for which still no effective, durable treatment exists [17]. In terrestrial mammals, comparative studies on the osteochondral unit have shown that the biochemical components of the tissues are strongly preserved across a wide range of species. However, a negative allometric relationship exists between cartilage thickness and body mass [18] and the trabecular bone structure is adapted to increasing body mass [14]. Nevertheless, comparative studies of the osteochondral unit of terrestrial and aquatic mammalian species do not exist, despite the high clinical relevance of the structure and the fundamental insight such studies might provide.

The aim of this study was to investigate if and how the structure of both the cartilage and the bone component of the osteochondral unit differ with 
relation to life on land or in water. Given that in both bone and cartilage, the prevalent structural function is absolved by the extracellular matrix and its architecture, the present work focused on how these differences impact the biomechanical properties of cartilage by analyzing the microstructural features of osteochondral units from the humeral head of six marine and nine terrestrial mammalian species.

\section{MATERIALS AND METHODS}

\section{Collection of materials and tissue harvest}

To investigate the morphology of cartilage tissue and of the interface between cartilage and bone, histological analysis was performed on osteochondral samples taken from fresh humeral heads of a variety of terrestrial and aquatic mammals (Table 1). Osteochondral tissue samples were harvested post-mortem from the weight bearing central area of the humeral head of adult animals sent for necropsy to the Pathology division, Faculty of Veterinary Medicine, Utrecht University, the Netherlands. For aquatic large species, samples were harvested on site directly from animals that were found

\begin{tabular}{|c|c|c|c|c|c|}
\hline & Species & Average body mass (kg) & Histology (n) & Micro-CT (n) & Bio-mechanics (n) \\
\hline 1 & Rat (Rattus sp) & 0.26 & 4 & 3 & - \\
\hline 2 & European Badger (Meles meles) & 13.2 & 1 & 1 & 1 \\
\hline 3 & Tufted deer (Elaphodus cephalophus) & 23 & 1 & - & - \\
\hline 4 & Indian crested porcupine (Hystrix indica) & 24.5 & 1 & 1 & - \\
\hline 5 & Cheetah (Acinonyx jubatus) & 25.5 & 1 & 1 & 1 \\
\hline 6 & Harbour porpoise (Phocena phocena) & 54.5 & 9 & 9 & 9 \\
\hline 7 & Harbour seal (Phoca vitulina) & 67.5 & 2 & 2 & 2 \\
\hline 8 & Shetland pony (Equus ferus caballus) & 175 & 2 & 2 & - \\
\hline 9 & Striped dolphin (Stenella coeruloalba) & 180 & 1 & 1 & 1 \\
\hline 10 & Onager (Equus hemionus) & 203 & 1 & 1 & - \\
\hline $8 b$ & Horse (Equus ferus caballus) & 550 & 2 & 2 & - \\
\hline 11 & Rothschild's giraffe (Giraffa camelopardalis) & 1070 & 2 & $2(1)$ & 2 \\
\hline 12 & White rhinoceros (Ceratotherium simum) & 1475 & 1 & 1 & - \\
\hline 13 & $\begin{array}{l}\text { Common minke whale (Balaenoptera } \\
\text { acutorostrata) }\end{array}$ & 5100 & 2 & - & - \\
\hline 14 & Sperm whale (Physeter macrocephalus) & 47.300 & 3 & 3 & 3 \\
\hline \multirow[t]{2}{*}{15} & Blue whale (Balaenoptera musculus) & 100.000 & 1 & 1 & 1 \\
\hline & Total & & 34 & 30 & 20 \\
\hline
\end{tabular}

Table 1. List of species included in the study. In total tissue samples from 84 animals (34 for histological analysis, 30 for micro-CT and 20 for biomechanical analysis) were harvested for a total of 15 different species. 
dead on the coasts of the Netherlands.

Animal species, age and body mass were recorded (or estimated in the case of whales based on species and body size), and macroscopic pictures of the joints were taken. Joints demonstrating macroscopic or microscopic signs of cartilage degeneration were excluded; animals displaying signs of incomplete endochondral ossification were identified as skeletally immature and excluded as well.

In total 84 tissue samples (34 for histology and polarized light microscopy, 30 for micro-CT and 20 for biomechanical testing) were harvested from mammals belonging to 15 different species, 9 terrestrial and 6 marine (Table 1); samples for histology were fixed in formalin $4 \%$, while samples for micro-CT analysis were stored in $70 \%$ ethanol; samples for biomechanical testing were frozen immediately after harvest in Tissue-tek ${ }^{\circledR}$ (Sakura Finitek, USA) until use.

\section{Histological preparation and analysis}

Samples were fixed using 4\% formalin, decalcified with Formical-2000 (EDTA/ formic acid; Decal Chemical Corporation, Tallman, NY) , dehydrated, cleared in xylene, embedded in paraffin and cut with a microtome to yield $5 \mu \mathrm{m}$ sections. Sections were stained with fast green and Safranin-O for measurements of cartilage thickness (distance from the surface to the interface with the subchondral bone). Average thickness of cartilage tissue for each sample was determined by averaging 3 measurements per image taken from different locations of the section. Digital images were analyzed using Image J software [19].

\section{Polarized Light Microscopy}

To evaluate the orientation of the cartilaginous collagen network, Polarized Light Microscopy (PLM) was chosen for its capacity to visualize the orientation of anisotropic materials [20-22]. Histological sections were stained with picrosirius red to stain the collagen fibers of the cartilage extracellular matrix [23]. All microscopic images were acquired through an Olympus DP73 digital camera using Cell^F software (Matrix Optics, Malaysia). A combination of UANT and U-POT filters mounted on a light microscope (Olympus BX51, Olympus) was used for the PLM measurements. The two cross polarizers were used so that highly ordered collagen fibers that were perpendicular or tangential to the articular surface appeared bright or red, while fibers with 

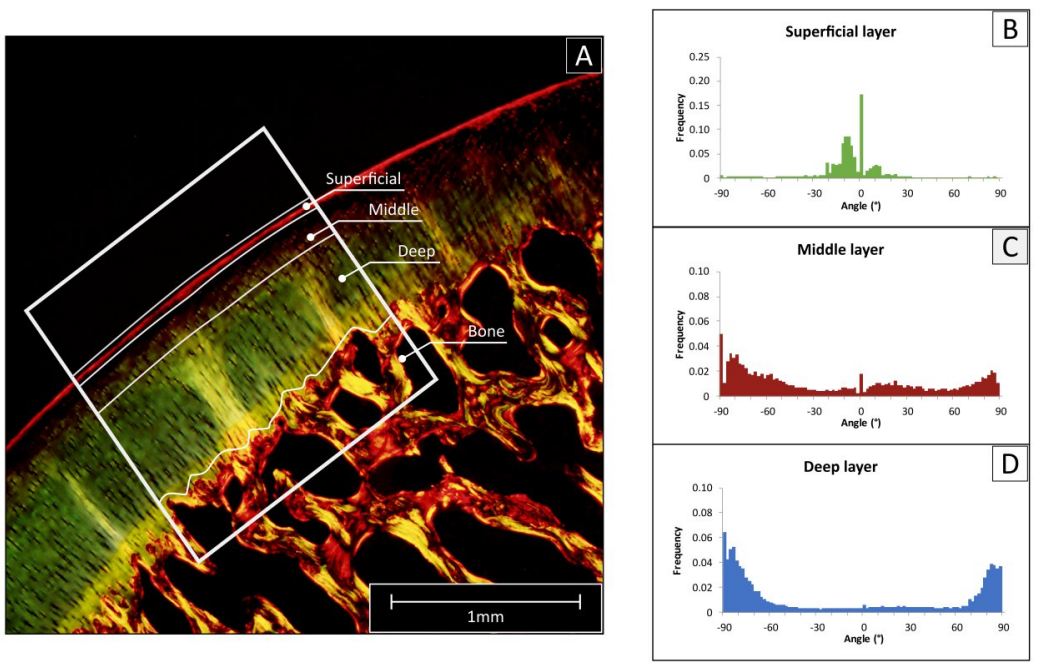

Figure 1. Use of polarized light microscopy (PLM) to determine collagen fiber orientation. (A) The histological sections stained with picrosirius red were imaged, then a region of interest was selected (ROI, white frame), and the cartilage tissue was divided into deep, middle and superficial layer. $(B-D)$ The selected region was analyzed with Image $J$ to determine the frequency of fibers for angle increments of $2^{\circ}$, obtaining a detailed histogram for each region.

other orientations (non-birefringent) appeared darkest. Collagen fiber orientation visualized via PLM was used for the classification of the superficial, middle and deep layer of cartilage $[20,24]$.

The area with the minimum birefringence value was identified as the border between the superficial and the middle zones, whereas the deep zone was considered to begin when the orientation angle values reached a plateau (typically close to 90 degrees with respect to cartilage surface) $[25,26]$. Micrographs acquired via PLM were converted to 8-bit images and the angle of orientation of the collagen structures was calculated within each of the three zones via automated image analysis and Fourier spectrum analysis, using the Directionality plug-in from the Fiji software [27]. Data were plotted as histograms of frequency for each representative angle, and as radial plots $\left(0^{\circ}\right.$ represent fibers parallel to the articulating surface) (Fig. 1). For each of the histograms, the peaks centered at $0^{\circ}$ and $\pm 90^{\circ}$ and were integrated to calculate their area using the OriginPro 8 software package (OriginLab, USA). Finally, cartilage relative layer thicknesses (\%) were calculated based on the 
data obtained with PLM.

\section{Biomechanical testing and data analysis}

The mechanical properties of the native cartilage of the osteochondral plugs of the different species were assessed by uniaxial unconfined compression using a universal testing machine (Zwick Z010, Germany) equipped with a $20 \mathrm{~N}$ load cell. Stress relaxation tests were performed by first applying a pre-load of $0.01 \mathrm{~N}$ to test samples and then strained to $15 \%$ at a rate of $10 \mu \mathrm{m} / \mathrm{s}$, followed by a relaxation period of $900 \mathrm{~s}$. Tests were conducted on cylindrical osteochondral plugs (Table S1). Prior to testing, samples were fixed to the bottom of a custom-made polycarbonate container using a cyanoacrylatebased adhesive and all tests were performed in PBS to approximate physiological conditions.

Peak and equilibrium stresses were calculated from each engineered stressstrain relaxation curve at peak or equilibrium, respectively. Here, stress is defined as the force divided by the specimen's unloaded cross-sectional area, and strain as the ratio between the original sample thickness and the displacement of compression platen. To quantify the relaxation response, a piecewise exponential function was fitted to the obtained stress-time curves, following the method by Castilho et al. [28]. Briefly, the fitted curves consist of three segments corresponding to an initial loading phase, and a fast and a slow relaxation phase. The formulae for the exponential curves for the fast and slow relaxation phases used are as follows,

$$
\sigma(t)=\left\{\begin{array}{l}
A_{1} e^{-t / \tau_{1}}+B_{1}, t<100 \\
A_{2} e^{-t / \tau_{2}}+B_{2}, t \geq 100
\end{array}\right.
$$

where the coefficients $A$ and $B$ are statistical parameters quantifying the shape of the relaxation curves and $\tau$ is a time constant that determines the rate of stress relaxation. The fitting procedure used minimizes the root mean square error between these curves and the measured stress, subject to the constraints that the fitted curves match the peak and equilibrium stress and that the two curves match at $\mathrm{t}=100 \mathrm{~s}$. 


\section{Micro-Computed Tomography}

For the detailed analysis of the microstructural features of the subchondral and trabecular bone, Micro Computed Tomography (micro-CT) was selected for the accurate measurement of micron-sized structures that constitute the bony tissue [29, 30]. Micro-CT images of the osteochondral cores were obtained with a micro-CT scanner (Quantum FX, Perkin Elmer, USA, voxel size = $20 \mathrm{\mu m}^{3}$ ). The automatically reconstructed micro-CT images were subsequently converted to series of 2D TIFF images and were binarized using local thresholding (Bernsen technique). BoneJ software [13] was used to determine the trabecular thickness (Tb.Th) and the bone volume fraction (Tb BV/TV) of the bone immediately underneath the cartilage (subchondral bone), and at the center of the bone of the osteochondral cores (trabecular bone).

\section{Statistical analysis}

Comparisons between multiple groups were performed using a one-way ANOVA combined with post-hoc t-tests with Bonferroni correction. A one-way ANOVA with Tukey's post hoc test was used for comparison in biomechanical analysis. A regression analysis using a power curve fit was performed for correlations between body mass and the parameters of cartilage thickness, peak modulus, trabecular thickness, and trabecular BV/TV. When applied, normality and homogeneity were checked with Shapiro-Wilks and Levene's tests. Limit of statistical significance was set at $p<0.05$.

\section{RESULTS}

\section{Histological and PLM analysis of cartilage}

Articular cartilage thickness varied widely between species, ranging from 209 $\mu \mathrm{m}$ in the mouse to $7660 \mathrm{~mm}$ in the sperm whale. Total cartilage thickness correlated with body mass (BM) with a negative allometric relationship $\left(R^{2}=0.91\right.$, a=0.28, Fig. 2A). This relationship was maintained when looking separately at terrestrial ( $R^{2}=0.90, \mathrm{a}=0.25$, Fig. $2 \mathrm{~B}$, green) and aquatic mammals $\left(R^{2}=0.90, \mathrm{a}=0.27\right.$, Fig. $2 \mathrm{~B}$, blue). Relative layer thickness (expressed as percentage of total thickness) in terrestrial and aquatic mammals was compared. The analysis showed significant differences in relative layer thickness, with the deep layer being relatively thicker in terrestrial than in aquatic mammals, and the middle layer being relatively thicker in aquatic 


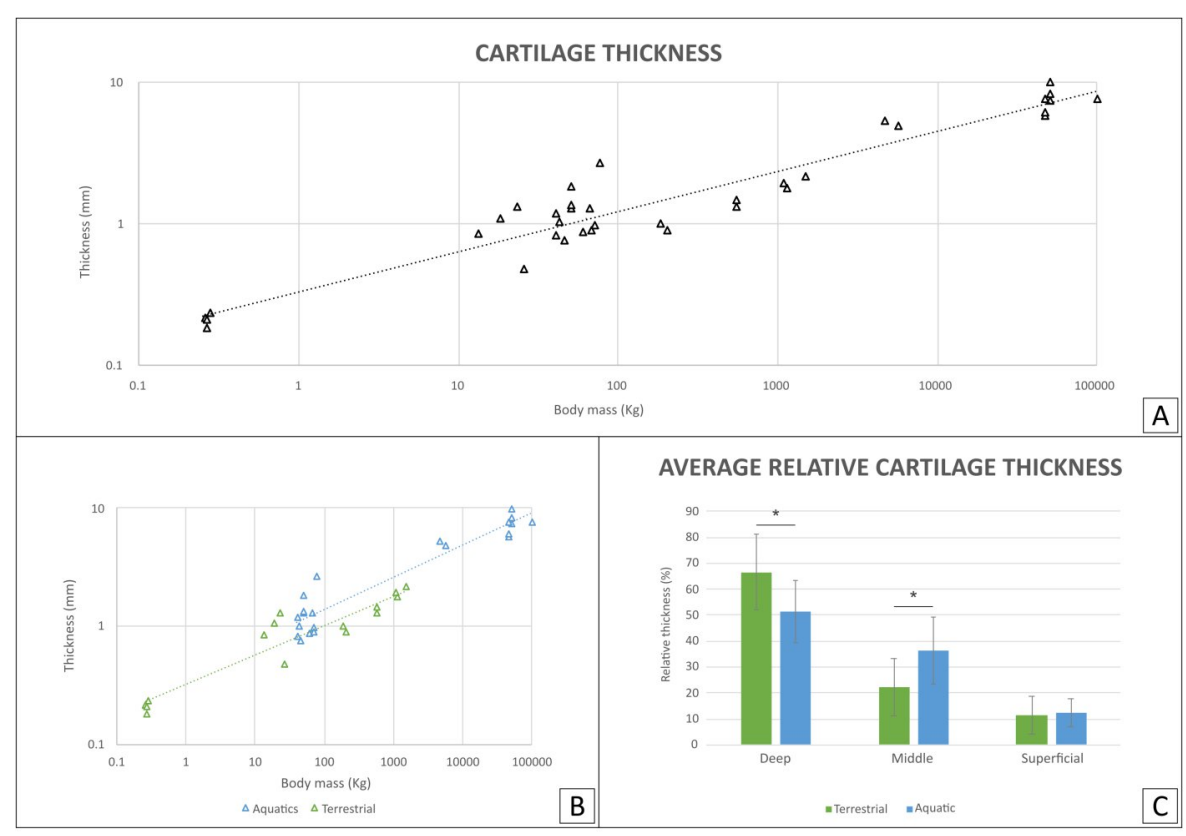

Figure 2. (A) Total cartilage thickness correlated with body mass with a negative allometric relationship $\left(R^{2}=0.91, a=0.28\right)$. (B) Total cartilage thickness in correlation with body mass for terrestrial and aquatic mammals separately. In green are terrestrial mammals $\left(R^{2}=0.90, a=0.25\right)$, in blue are aquatic mammals $\left(R^{2}=0.91, a=0.27\right)$. (C) Average of relative layer thickness (\%) was calculated for each cartilage layer in terrestrial (green) and aquatic (blue) mammals. Comparison showed a significant difference between the two groups in both the deep and middle layers $(p<0.01)$.

mammals than in terrestrial mammals. No significant differences were found in superficial layer relative thickness (Fig. 2C).

With PLM the visualization of the different collagen fiber orientations (Fig. $3 A-C)$ was possible, showing fibers being predominantly oriented perpendicularly to the surface in the deep layer and parallel to the surface in the superficial layer in terrestrial mammals, and no predominant orientation of fibers in aquatic mammals (Fig. 3D-E).

\section{Biomechanical analysis}

Characterization of biomechanical behavior showed a marked difference in the stress relaxation curve of aquatic and terrestrial cartilage samples (Fig. 4A). Analysis of time dependent mechanical properties showed that peak modulus was significantly higher in terrestrial mammals than in aquatic mammals (Fig. 4C, $p<0.05$ ), while at equilibrium, cartilage appeared to be slightly stiffer in 


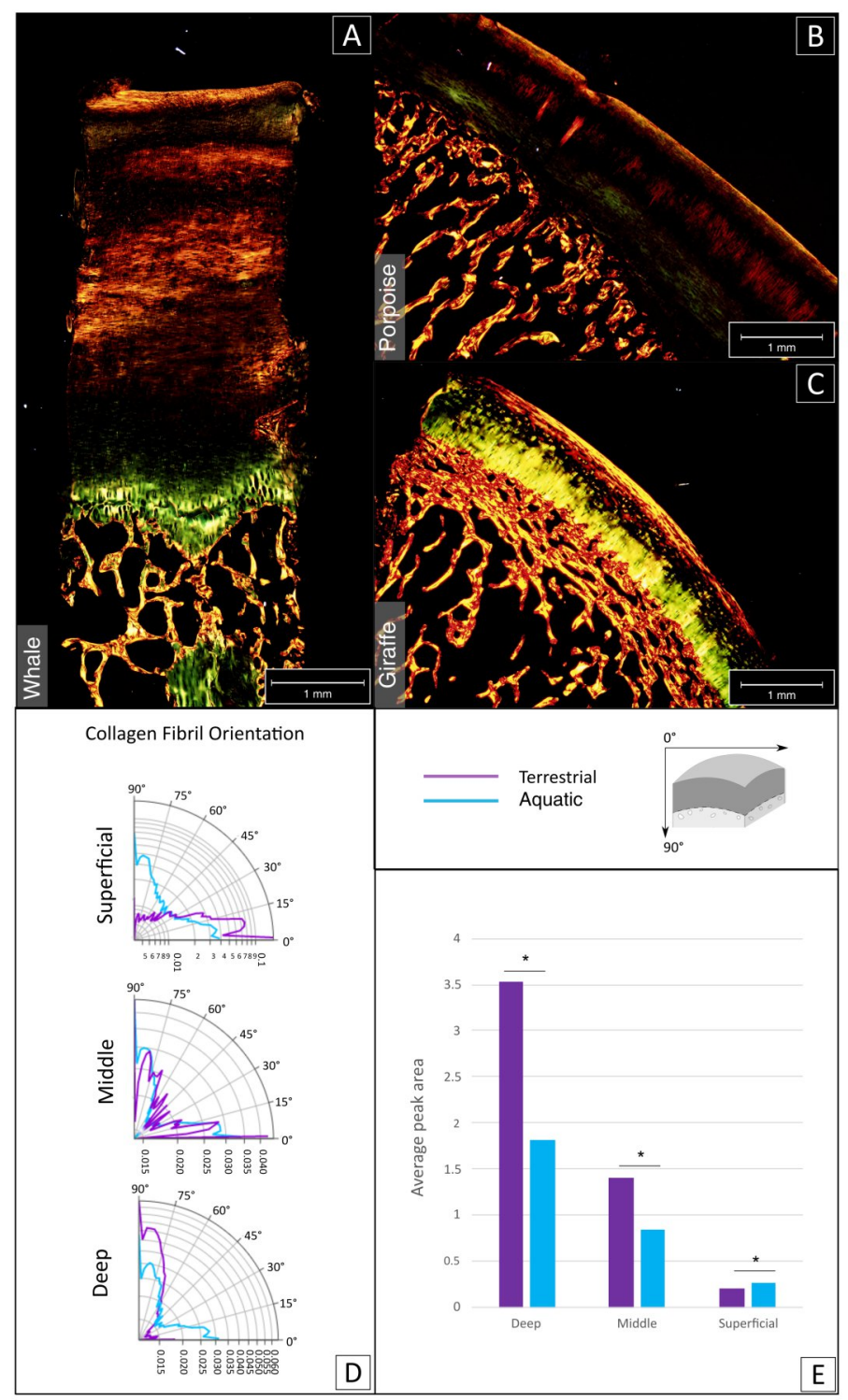

Figure 3. (A-C) Examples of histological sections stained with picrosirius red and imaged with $P L M$. The collagen fiber orientation of aquatic mammals $(A, B)$ appeared less organized than fiber orientation in terrestrial mammals (C). (C) Cartilage structure in terrestrial mammals featured a clear distinction between deep, middle, and superficial layer (respectively in yellow, black, and red/yellow from bone to surface). (D) Polar graphical representation of collagen fiber distribution for deep, middle, and superficial layer in terrestrial (violet) and aquatic (cerulean) mammals. $O^{\circ}$ are fibers parallel to the surface, $90^{\circ}$ are fibers perpendicular to the surface. (E) Average peak area was calculated for each cartilage layer in both terrestrial (violet) and aquatic (cerulean) mammals, showing significant differences between the two groups in all layers $(p<0.05)$. 


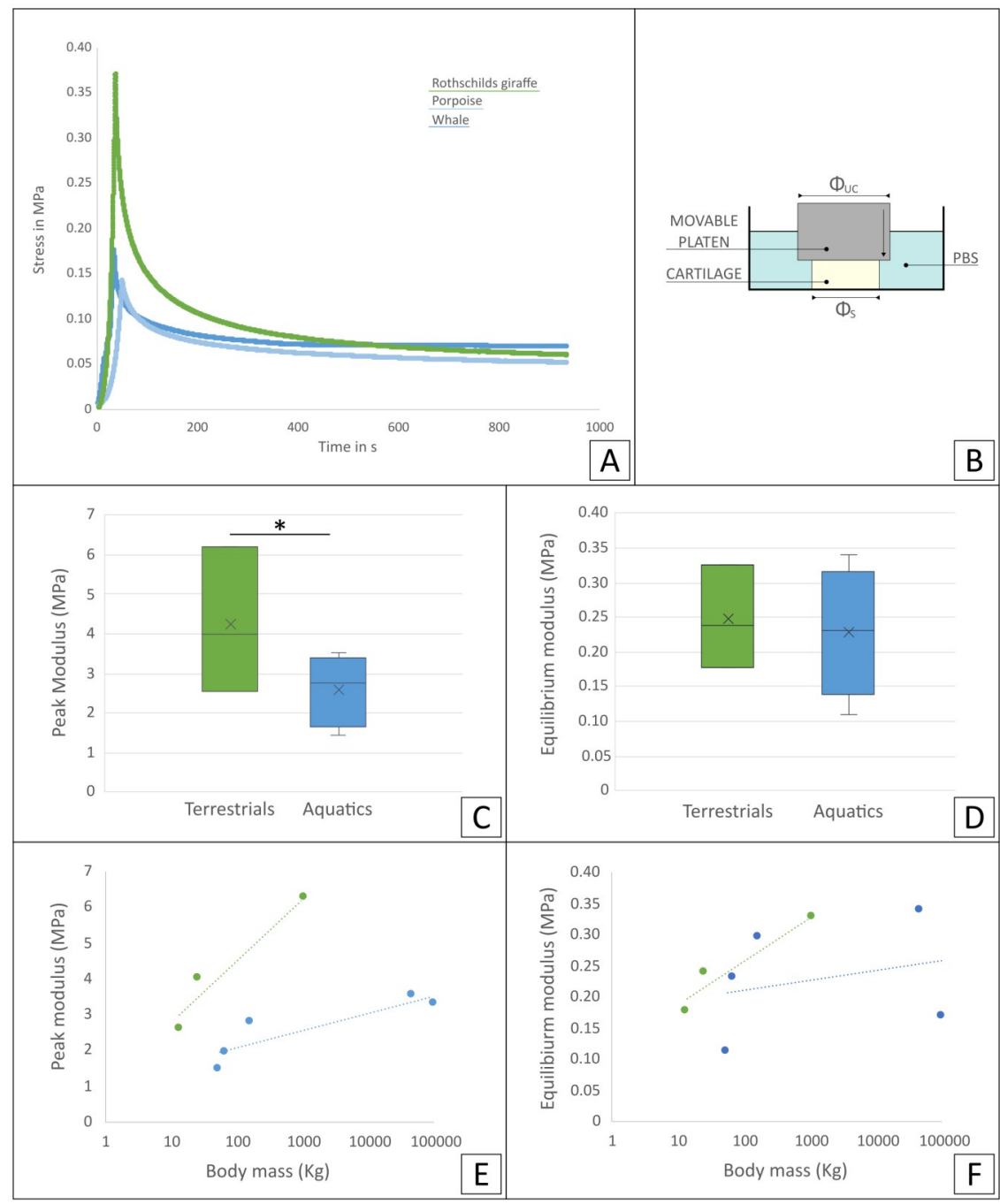

Figure 4. (A) Representative stress relaxation curve of tested cartilage samples. (B) Schematics of loading set-up: unconfined compression geometry (congruent loading) used on cartilage samples immersed in a PBS bath. Comparison of time dependent mechanical properties: (C) peak and (D) equilibrium modulus for terrestrial (green) and aquatic mammals (blue). * Indicates a significant difference. Relationship of (E) peak and (F) equilibrium moduli average for each species with body mass for terrestrial (green) and aquatic mammals (blue).

terrestrial mammals (Fig. 4D). Initial stress relaxation appeared slower for aquatic mammals and steeper for terrestrial mammals (Fig. S1A). No significant differences were observed in the phase of slow relaxation (Fig. S1B). Comparison of the relationship of peak modulus and body mass (Fig. 4E, F). 


\section{Micro-Computed Tomography}

Imaging through histology and micro-CT showed that in aquatic mammals (Fig. 5A, B) the interface between the hyaline articular cartilage and the subchondral bone did not consist of the layer of calcified cartilage and dense subchondral plate, as typical for terrestrial mammals (Fig. 5C, D). There was no calcified layer and there was less dense, more porous subchondral bone in aquatic mammals (Fig. 5E). Bone volume over total volume (BV/TV) in the subchondral area (immediately underneath the interface) averaged at $80.43 \pm$ $16.75 \%$ for aquatic mammals, which was significantly lower than in terrestrial mammals ( $98.52 \pm 0.81 \%$, $p<0.01$, Fig. $5 F$ ).

The trabecular bone below the subchondral plate under the central loading area of the humeri was denser, with thicker trabeculae in terrestrial mammals (34.52 $\pm 11.09 \%$ for terrestrial and $29.36 \pm 10.31 \%$ for aquatic mammals) (Fig. $6 \mathrm{~A}, \mathrm{~B})$. In aquatic mammals living in shallow waters bone was considerably denser (Fig. 6C, D) compared to deep diving mammals (34.95 $\pm 5.3 \%$ for seals
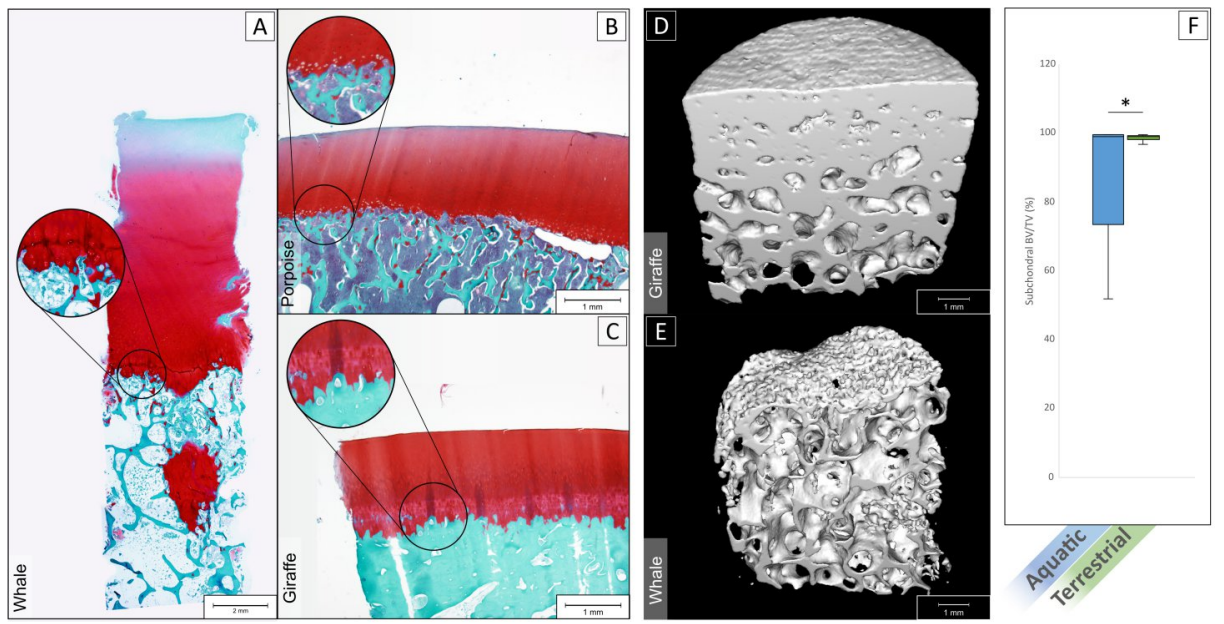

Figure 5. (A-C) Histological sections stained with safranin-O of a whale $(A)$, porpoise $(B)$ and giraffe (C). Aquatic mammals $(A, B)$ showed a continuous transition from cartilage to bone. Terrestrial mammals (C) displayed the characteristic calcified cartilage layer with tidemark (in magnification bubble). (D) 3D rendered micro-CT scan of giraffe bone core, showing on top a compact surface interfacing with cartilage (not shown in scan) and a gradually more porous structure as the subchondral tissue transitions to trabecular bone. (E) 3D rendered micro-CT scan of a whale bone core, showing a porous surface on top where it interfaces with cartilage, and a porous structure underneath with seemingly no subchondral bone transition before the trabecular bone. (F) Bone volume/total volume (BV/TV) in the subchondral area (immediately underneath the cartilage) for aquatic mammals (blue) was significantly lower than in terrestrial mammals (green) $(p<0.01)$. 
and porpoises, $13.97 \pm 2.75 \%$ for whales) (Fig. 6E, F). Trabecular thickness averaged at $211.71 \pm 141.08 \mu \mathrm{m}$ in terrestrial mammals and at $120.01 \pm 27.85$ $\mu \mathrm{m}$ in aquatic mammals. Trabecular BV/TV increased with size in terrestrial mammals ( $R^{2}=0.44$, Fig. $6 \mathrm{G}$, green) and decreased with size in aquatic mammals ( $R^{2}=0.86$, Fig. $6 \mathrm{G}$, blue). Thickness of the bone trabeculae (Tb.Th) increased with size in terrestrial mammals $\left(R^{2}=0.65, \mathrm{a}=0.12, \mathrm{Fig} .6 \mathrm{H}\right)$, but was independent of size in aquatic mammals.

\section{DISCUSSION}

The present study investigated how osteochondral tissue has differentiated in terrestrial and aquatic mammals to accommodate the different demands of life and locomotion on land or in water. Here, the characteristics of articular cartilage of aquatic mammals are described for the first time. In contrast to terrestrial mammals, cartilage collagen fibers were revealed not to display a
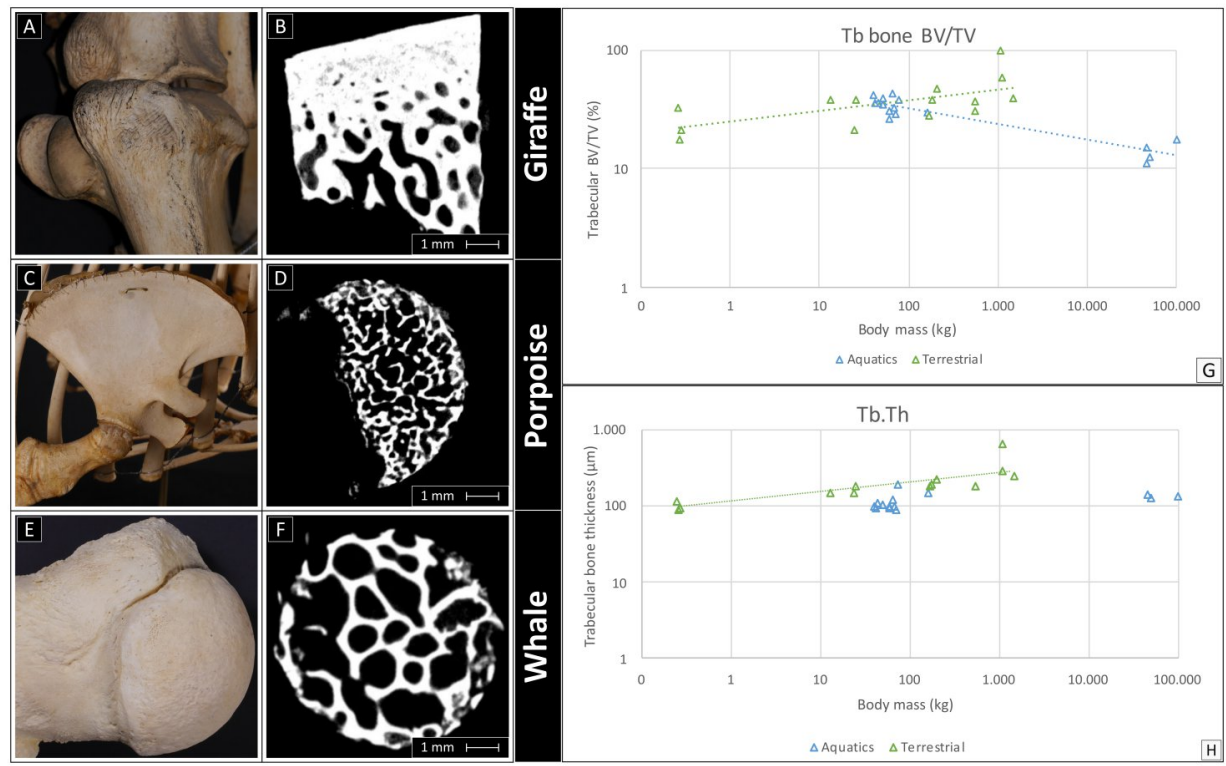

Figure 6. Examples of macroscopic (left) and micro-CT scans (right) of a giraffe, porpoise, and whale. Left images show the macroscopic aspect of the humeral head of two aquatics and a terrestrial animal, on the right the correspondent micro-CT scan of the trabecular bone. (A, B) Terrestrial mammals featured a denser bone, while bone density was lower in porpoises $(C, D)$ and even lower in deep-diving animals (E, F). (G) Trabecular BV/TV increased with body mass in terrestrial mammals (green), and decreased with body mass in aquatic mammals (blue). (H) Trabecular bone thickness (Tb.Th) increased with size in terrestrial mammals (green), and was independent of size in aquatic mammals (blue). 
preferential alignment. Moreover, the cartilage-bone interface of aquatic mammals lacks the typical calcified layer and has a much less dense subchondral plate, featuring a more continuous transition from cartilage to relatively porous trabecular bone with thinner trabeculae. These trabeculae do not increase in size with increasing body mass, in contrast with what has been observed in terrestrial mammals.

The findings in the current study add significantly to our knowledge on the basic aspects of the biology of the osteochondral unit and clearly reflect the crucial influence of loading. This increased insight in the basic biology of the different structural components of the osteochondral unit is of great importance from a tissue repair perspective, as it guides which considerations should be prioritized in the quest for recapitulation of the native template for regenerative purposes.

Previous research has vastly expanded our knowledge on cartilage tissue over a wide range of terrestrial mammals. It has shown, for instance, that cartilage biochemistry is remarkably preserved among species, and that the tissue displays microstructural changes that allow functionality in mice as much as in elephants [18]. One of the features that is key in the accommodation of different forces generated by body mass is tissue thickness. Malda et al. reported that articular cartilage thickness scaled with negative allometry in relationship to body mass $(a=0.28)$, based on the analysis of nearly 60 terrestrial mammal species [18]. Interestingly, in the analysis presented here, it was observed that this correlation was similar in aquatic mammals, suggesting that this relationship might be dependent of factors other than loading, such as possibly metabolic limitations. In hyaline cartilage, an avascular tissue in which nutrient supply is dependent on diffusion and fluid flow across a poroelastic matrix [31], metabolite limitation is known to be related to tissue thickness $[18,32]$. Simon et al. did not observe a consistent correlation between cartilage thickness and estimated compressive stress on the joint in a study conducted on 5 species of terrestrial mammals that varied widely in body mass (mouse, rat, dog, sheep and cow [33]. When looking into more detail at the thickness of the different zones that make up articular cartilage, there were significant differences between aquatic and terrestrial mammals in the deep and middle layers, but not in the superficial layer. This supports the notion that the primary role of the superficial layer of cartilage is to distribute impact forces and loads among the tissue [25, 34] and load 
distribution of forces is important in both sea-borne animals, as it is in terrestrial species. Compressive loads will, however, be lower in marine species, which can explain the observation that there is a relationship with body mass of the thickness of the middle and deep layers in terrestrial species $[14,35]$, but not in aquatic mammals.

A significant difference in the predominance of fiber orientation was found between aquatic and terrestrial mammals in all cartilage layers: collagen fiber orientation in terrestrial species followed the classic Benninghoff arcade model [36] in which orientation is principally parallel to the surface in the superficial layer, and in the middle zone bending towards an orientation perpendicular to the subchondral bone in the deep layer. Conversely, in aquatic mammals, orientation of the collagen fibers in the deep or middle zones was random. It can be assumed that the absence of gravity strongly reduces compressive loading of joints and, to a large extent eliminates the need for a collagen orientation as seen in terrestrial mammals. However, it remains unclear whether this heterogeneity in the alignment of the collagen fibers is merely due to the absence of the need for a specifically organized orientation, or whether it reflects the specialization of the joint to sustain the physical forces, which will be principally shear forces, that are generated by the action of swimming [5, 37-39].

The different collagen fiber orientation observed in the aquatic mammals compared to terrestrial mammals is reflected as well in the biomechanical behavior of cartilage, as shown by the unconfined compression tests: the significantly higher peak and equilibrium moduli in terrestrial mammals are most likely linked to the existence of the Benninghoff arcades in those species, providing resistance against compressive loading, which is virtually absent in aquatic mammals. The mechanical responses observed in the aquatic mammals are indicative of an overall less stiff tissue, compared to terrestrial mammal. Furthermore, the absence of a defined collagen network structure in the aquatic mammals is likely to provide less resistance to osmotic swelling, as in terrestrial mammal's cartilage up to two thirds of the elastic modulus in compression arises from the electrostatic contributions and osmotic swelling that is restricted inside the organized collagen matrix [40,41].

The lack of a calcified cartilage layer and a compact subchondral plate, which are typical features of the osteochondral unit in terrestrial animals [8], was remarkable. The main function of the calcified layer in the osteochondral unit 
is to provide a gradual transition in stiffness from hyaline cartilage to subchondral bone and to serve as anchor point for the collagen fibers in the deep layer of the hyaline cartilage, i.e. to serve as a foundation for the Benninghoff arcades [8, 42, 43]. The subchondral plate has a role in distributing the compressive forces acting on the joint. In aquatic mammals, compressive loading is of minor importance and it may well be that the anchoring of the collagen fibrils is much less important for this reason. Detailed studies using electron microscopy might shed some light on this issue. The cartilage/bone interface of aquatic mammals was more porous than in terrestrials, with an almost trabecular-like subchondral bone that displayed a great variability in bone volume/total volume in the different marine species. The increased porosity, and thus the greater potential for transfer of solutes, may permit the great cartilage thickness seen in some aquatic mammals (over $7 \mathrm{~mm}$ in the largest whale). Cartilage is, as an avascular tissue, dependent on diffusion, so critical thickness will be larger if diffusion of nutrients can be provided from two sides. The ratio of nutrient exchange is likely to be different in aquatic mammals, as at the few sites where the hyaline cartilage is in direct contact with the subchondral bone (in terrestrial animals), solute exchange has been shown to be five-fold larger than through the calcified cartilage [44].

Bone density deeper under the cartilage tissue (trabecular BV/TV) was relatively higher in terrestrial mammals when size increased, and relatively lower in aquatic mammals. The first observation is, again, related to loading and is in accordance with earlier research, that has illustrated how in terrestrial mammals trabecular bone increases in bone volume/total volume as animals increase in size [45]. The second observation, is attributable to the lack of loading and the fact that in aquatic mammals living in deep waters, low bone density enables dynamic buoyancy control [15]. According to Dumont et al., bone microanatomy carries an ecological signal that holds information about habitat and locomotion patterns, which is particularly evident in tetrapods that have developed advanced secondary adaptations to life in water [46]. In the matter of buoyancy control of aquatic mammals, species recently adapted to aquatic life or living in shallow waters have bones that are denser than in terrestrial forms to allow static control (ballast), as opposed to a lower bone density typically associated with the dynamic buoyancy control required by animals that exhibit deep diving behavior for longer periods [15, 47]. With respect to trabecular thickness, this parameter also increased with 
size in terrestrial mammals, with a trend similar to previous reports in literature $[14,48]$, while in aquatic mammals trabecular thickness appeared to scale independently. This independence can possibly be explained by the lack of compressive forces necessary to elicit a proportionate response in the bone for trabecular thickness as suggested by Wolff's law [49, 50]. Rolvien et al. investigated several bone parameters in the vertebral bodies of three whale species (sperm whale, orca and harbor porpoise), and found in comparison, that bone volume fraction (BV/TV) did not scale with body size but that trabeculae were thicker and fewer in number in larger whale species [51]. Although research has shown the relationship between bone microanatomical organization, habitats and locomotion patterns in different taxa [46], when going into deeper detail (as for example between different species of whales), the interconnections between habitat, locomotion patterns and bone become less clear, particularly in view of the variability of diving-depth behavior of different animals [51], which could explain these diverging findings.

Curiously, the histological sections of aquatic mammals, displayed small islands of cartilaginous tissue still embedded within the bone, and in the larger aquatic species, such as the blue whale and the sperm whale, large islands of cartilage tissue were found deep in the trabecular bone. These appeared as large lacunae upon first inspection with micro-CT, and our initial explanation was a possible area of osteocyte death due to barotraumas, which have been anecdotally described in deep-diving mammals [52]. Upon histological evaluation, these islands appeared to be composed of healthy cartilage-like tissue naturally embedded in the bone, devoid of evident signs of bone remodeling, not suggesting any form of pathology. The areas may represent incomplete osteochondral ossification, well-known in the horse and other species as osteochondrosis [53, 54] similarly to what was found and described by Rolvien et al. in the central areas of the vertebrae of sperm whales [51]. However, our pathologist was more inclined towards the finding being the result of possible noxious insults.

This study highlighted a number of important differences in the microstructure and biomechanical characteristics of the osteochondral unit between aquatic and terrestrial mammals. Where the research would have benefitted from a larger pool of animals, particularly with regards to aquatic mammals, the findings are unambiguous and all point at the great importance of loading for articular tissues. The many and large differences between the 
osteochondral units between terrestrial animals and aquatic mammals could all be attributed to the very different loading conditions in their respective environments. This stresses the crucial role loading plays in joint function and supports the recent call for rethinking the current paradigms in articular cartilage regeneration [55].

\section{ACKNOWLEDGMENTS}

The authors would like to acknowledge the Marine Mammals Team from the Department of Biomedical Health Sciences, Pathology division, Veterinary Medicine and R. Wagensveld-van den Dikkenberg for their valuable help with the work on field and support with tissue collection. The authors would also like to thank P. Kamminga and the Naturalis Biodiversity Center in Leiden, Netherlands, for access to their collection of mammalian skeletons.

The research leading to these results has received funding from the European Community's Seventh Framework Programme (FP7/2007- 2013) under grant agreement 309962 (HydroZONES), and the Dutch Arthritis Association (ReumaNL, LLP-12 and LLP-22). 


\section{REFERENCES}

[1] M.D. Uhen, Evolution of marine mammals: back to the sea after 300 million years, The Anatomical Record: Advances in Integrative Anatomy and Evolutionary Biology: Advances in Integrative Anatomy and Evolutionary Biology 290(6) (2007) 514-522.

[2] J.S. Reidenberg, Anatomical adaptations of aquatic mammals, The Anatomical Record: Advances in Integrative Anatomy and Evolutionary Biology: Advances in Integrative Anatomy and Evolutionary Biology 290(6) (2007) 507-513.

[3] B. Cozzi, S. Mazzariol, M. Podestu, A. Zotti, Diving adaptations of the cetacean skeleton, Open Zoology Journal 2 (2010) 24-32.

[4] J. Thewissen, M. Cohn, L. Stevens, S. Bajpai, J. Heyning, W. Horton, Developmental basis for hind-limb loss in dolphins and origin of the cetacean bodyplan, Proceedings of the National Academy of Sciences 103(22) (2006) 8414-8418.

[5] P. Dejours, Water and air physical characteristics and their physiological consequences, Comparative physiology: Life in water and on land 9(3) (1987).

[6] B.S. Turnbull, D.F. Cowan, Synovial joint disease in wild cetaceans, Journal of wildlife diseases 35(3) (1999) 511-518.

[7] C.E. Farnum, Postnatal Growth of Fins and Limbs through Endochondral Ossification, Fins into limbs: evolution, development, and transformation (2007) 118.

[8] A.J. Sophia Fox, A. Bedi, S.A. Rodeo, The basic science of articular cartilage: structure, composition, and function, Sports health 1(6) (2009) 461-468.

[9] J.A. Sanchez, A. Berta, Comparative anatomy and evolution of the odontocete forelimb, Marine mammal science 26(1) (2010) 140-160.

[10] J. Thewissen, Musculature, Encyclopedia of marine mammals, Elsevier2009, pp. 744-747.

[11] V. De Buffrenil, D. Schoevaert, On how the periosteal bone of the delphinid humerus becomes cancellous: ontogeny of a histological specialization, Journal of Morphology 198(2) (1988) 149-164.

[12] W.J. Felts, F.A. Spurrell, Structural orientation and density in cetacean humeri, American Journal of Anatomy 116(1) (1965) 171-203.

[13] M. Doube, M.M. Kłosowski, I. Arganda-Carreras, F.P. Cordelières, R.P. Dougherty, J.S. Jackson, B. Schmid, J.R. Hutchinson, S.J. Shefelbine, BoneJ: free and extensible bone image analysis in ImageJ, Bone 47(6) (2010) 1076-1079.

[14] I. Mancini, L. Rieppo, B. Pouran, I. Afara, F.S. Braganca, M. van Rijen, M. Kik, H. Weinans, J. Toyras, P. van Weeren, Effects of body mass on microstructural features of the osteochondral unit: A comparative analysis of 37 mammalian species, Bone 127 (2019) 664-673.

[15] N.M. Gray, K. Kainec, S. Madar, L. Tomko, S. Wolfe, Sink or swim? Bone density as a mechanism for buoyancy control in early cetaceans, The Anatomical Record: Advances in Integrative Anatomy and Evolutionary Biology: Advances in Integrative Anatomy and Evolutionary Biology 290(6) (2007) 638-653.

[16] M.A. Taylor, Functional significance of bone ballastin in the evolution of buoyancy control strategies by aquatic tetrapods, Historical Biology 14(1-2) (2000) 15-31.

[17] S.I. Lepage, N. Robson, H. Gilmore, O. Davis, A. Hooper, S. St. John, V. Kamesan, P. Gelis, D. Carvajal, M. Hurtig, Beyond cartilage repair: the role of the osteochondral unit in joint health and disease, Tissue Engineering Part B: Reviews 25(2) (2019) 114-125.

[18] J. Malda, J.C. de Grauw, K.E. Benders, M.J. Kik, C.H. van de Lest, L.B. Creemers, W.J. Dhert, P.R. van Weeren, Of mice, men and elephants: the relation between articular cartilage thickness and body mass, PloS one 8(2) (2013).

[19] J. Schindelin, Fiji: An open-source platform for biological-image analysis. Nature Methods9, 676-682.

[20] A. Changoor, N. Tran-Khanh, S. Methot, M. Garon, M. Hurtig, M. Shive, M. Buschmann, A polarized light microscopy method for accurate and reliable grading of collagen organization in cartilage repair, Osteoarthritis and cartilage 19(1) (2011) 126-135.

[21] Y. Yarker, R. Aspden, D. Hukins, Birefringence of articular cartilage and the distribution of 
collagen fibril orientations, Connective tissue research 11(2-3) (1983) 207-213.

[22] D.P. Speer, L. Dahners, The collagenous architecture of articular cartilage. Correlation of scanning electron microscopy and polarized light microscopy observations, Clinical orthopaedics and related research (139) (1979) 267-275.

[23] L.C.U. Junqueira, G. Bignolas, R.R. Brentani, Picrosirius staining plus polarization microscopy, a specific method for collagen detection in tissue sections, The Histochemical journal 11(4) (1979) 447-455.

[24] J. Rieppo, J. Hallikainen, J.S. Jurvelin, I. Kiviranta, H.J. Helminen, M.M. Hyttinen, Practical considerations in the use of polarized light microscopy in the analysis of the collagen network in articular cartilage, Microscopy research and technique 71(4) (2008) 279-287.

[25] P. Julkunen, P. Kiviranta, W. Wilson, J.S. Jurvelin, R.K. Korhonen, Characterization of articular cartilage by combining microscopic analysis with a fibril-reinforced finite-element model, Journal of biomechanics 40(8) (2007) 1862-1870.

[26] J. Arokoski, M.M. Hyttinen, T. Lapveteläinen, P. Takács, B. Kosztáczky, L. Módis, V. Kovanen, $\mathrm{H}$. Helminen, Decreased birefringence of the superficial zone collagen network in the canine knee (stifle) articular cartilage after long distance running training, detected by quantitative polarised light microscopy, Annals of the rheumatic diseases 55(4) (1996) 253.

[27] A. Sensini, C. Gualandi, A. Zucchelli, L.A. Boyle, A.P. Kao, G.C. Reilly, G. Tozzi, L. Cristofolini, M.L. Focarete, Tendon fascicle-inspired nanofibrous scaffold of polylactic acid/collagen with enhanced 3D-structure and biomechanical properties, Scientific reports 8(1) (2018) 1-15.

[28] M. Castilho, V. Mouser, M. Chen, J. Malda, K. Ito, Bi-layered micro-fibre reinforced hydrogels for articular cartilage regeneration, Acta biomaterialia 95 (2019) 297-306.

[29] R. Fajardo, R. Müller, Three-dimensional analysis of nonhuman primate trabecular architecture using micro-computed tomography, American journal of physical anthropology 115(4) (2001) 327-336.

[30] D.W. Holdsworth, M.M. Thornton, Micro-CT in small animal and specimen imaging, Trends in Biotechnology 20(8) (2002) S34-S39.

[31] X. Lu, V. Mow, Biomechanics of articular cartilage and determination of material properties, Medicine+ Science in Sports+ Exercise 40(2) (2008) 193.

[32] C.R. White, R.S. Seymour, Mammalian basal metabolic rate is proportional to body mass $2 / 3$, Proceedings of the National Academy of Sciences 100(7) (2003) 4046-4049.

[33] W.H. Simon, Scale effects in animal joints. I. Articular cartilage thickness and compressive stress, Arthritis \& Rheumatism: Official Journal of the American College of Rheumatology 13(3) (1970) 244-255.

[34] S. Bevill, A. Thambyah, N. Broom, New insights into the role of the superficial tangential zone in influencing the microstructural response of articular cartilage to compression, Osteoarthritis and Cartilage 18(10) (2010) 1310-1318.

[35] P. Brama, J. Tekoppele, R. Bank, D. Karssenberg, A. Barneveld, P. Van Weeren, Topographical mapping of biochemical properties of articular cartilage in the equine fetlock joint, Equine veterinary journal 32(1) (2000) 19-26.

[36] M. Kääb, I. Ap Gwynn, H. Nötzli, Collagen fibre arrangement in the tibial plateau articular cartilage of man and other mammalian species, The Journal of Anatomy 193(1) (1998) 23-34.

[37] A.R. Hoelzel, Marine mammal biology: an evolutionary approach, John Wiley \& Sons2009, p. 73.

[38] T.M. Williams, The evolution of cost efficient swimming in marine mammals: limits to energetic optimization, Philosophical Transactions of the Royal Society of London. Series B: Biological Sciences 354(1380) (1999) 193-201.

[39] T.M. Williams, Intermittent Swimming by Mammals: A Strategy for Increasing Energetic Efficiency During Diving1, American Zoologist 41(2) (2015) 166-176.

[40] W.M. Lai, J. Hou, V.C. Mow, A triphasic theory for the swelling and deformation behaviors of articular cartilage, (1991).

[41] C.C. Guterl, C.T. Hung, G.A. Ateshian, Electrostatic and non-electrostatic contributions of proteoglycans to the compressive equilibrium modulus of bovine articular cartilage, Journal of biomechanics 43(7) (2010) 1343-1350. 
[42] H. Madry, C.N. van Dijk, M. Mueller-Gerbl, The basic science of the subchondral bone, Knee surgery, sports traumatology, arthroscopy 18(4) (2010) 419-433.

[43] A. Benninghoff, Form und Bau der Gelenkknorpel in ihren Beziehungen zur Funktion, Zeitschrift für Anatomie und Entwicklungsgeschichte 76(1-3) (1925) 43-63.

[44] K. Arkill, C. Winlove, Solute transport in the deep and calcified zones of articular cartilage, Osteoarthritis and Cartilage 16(6) (2008) 708-714.

[45] M.M. Barak, D.E. Lieberman, J.-J. Hublin, of mice, rats and men: trabecular bone architecture in mammals scales to body mass with negative allometry, Journal of structural biology 183(2) (2013) 123-131.

[46] M. Dumont, M. Laurin, F. Jacques, E. Pelle, W. Dabin, V. de Buffrénil, Inner architecture of vertebral centra in terrestrial and aquatic mammals: a two-dimensional comparative study, Journal of Morphology 274(5) (2013) 570-584.

[47] W.P. Wall, The correlation between high limb-bone density and aquatic habits in recent mammals, Journal of Paleontology (1983) 197-207.

[48] M. Doube, M.M. Kłosowski, A.M. Wiktorowicz-Conroy, J.R. Hutchinson, S.J. Shefelbine, Trabecular bone scales allometrically in mammals and birds, Proceedings of the Royal Society B: Biological Sciences 278(1721) (2011) 3067-3073.

[49] H.M. Frost, From Wolff's law to the Utah paradigm: insights about bone physiology and its clinical applications, The Anatomical Record: An Official Publication of the American Association of Anatomists 262(4) (2001) 398-419.

[50] J. Wolff, Das Gesetz der Transformation der Knochen, DMW-Deutsche Medizinische Wochenschrift 19(47) (1893) 1222-1224.

[51] T. Rolvien, M. Hahn, U. Siebert, K. Püschel, H.-J. Wilke, B. Busse, M. Amling, R. Oheim, Vertebral bone microarchitecture and osteocyte characteristics of three toothed whale species with varying diving behaviour, Scientific reports 7(1) (2017) 1604.

[52] M.J. Moore, G.A. Early, Cumulative sperm whale bone damage and the bends, Science 306(5705) (2004) 2215-2215.

[53] H.W. Thomas, L.G. Barnes, The bone joint pathology osteochondrosis in extant and fossil marine mammals, Natural history museum of Los Angeles county2015.

[54] A.M. McCoy, F. Toth, N.I. Dolvik, S. Ekman, J. Ellermann, K. Olstad, B. Ytrehus, C.S. Carlson, Articular osteochondrosis: a comparison of naturally-occurring human and animal disease, Osteoarthritis and cartilage 21(11) (2013) 1638-1647.

[55] J. Malda, J. Groll, P.R. van Weeren, Rethinking articular cartilage regeneration based on a 250-year-old statement, Nature Reviews Rheumatology (2019) 1. 


\section{SUPPLEMENTAL DATA}

\begin{tabular}{lrrr}
\hline Species & Number of samples & Cartilage diameter $(\mathrm{mm})$ & Cartilage thickness $(\mathrm{mm})$ \\
\hline European Badger & 1 & 6.4 & 1.7 \\
Cheetah (Acinonyx jubatus) & 2 & 6.5 & 2.1 \\
Rothschild's giraffe (Giraffa camelopardalis) & 3 & 6.9 & 1.7 \\
Harbour Porpoise & 9 & 6.6 & 1.7 \\
Harbour Seal & 2 & 7.4 & 3.7 \\
Striped Dolphin & 3 & 7.6 & 1.9 \\
Sperm Whale & 4 & 6.3 & 8.5 \\
Blue Whale & 2 & 6.4 & 8.9 \\
\hline
\end{tabular}

Table S1. Overview of cartilage samples on which biomechanical tests were conducted, with average diameter and thickness per species.

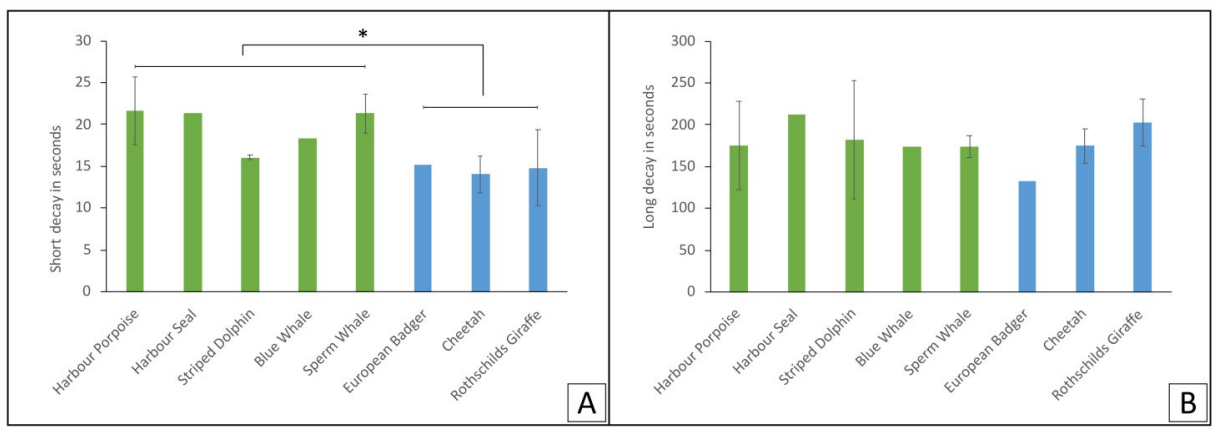

Figure S1. (A) Initial stress relaxation appeared slower for aquatic mammals and steeper for terrestrial mammals. (B) No significant differences were observed in the phase of slow relaxation. 


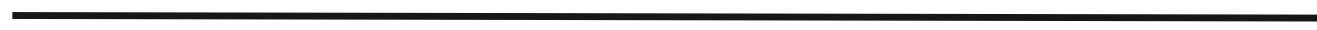


Translation of hydrogel-based regenerative strategies in the equine model for cartilage repair 


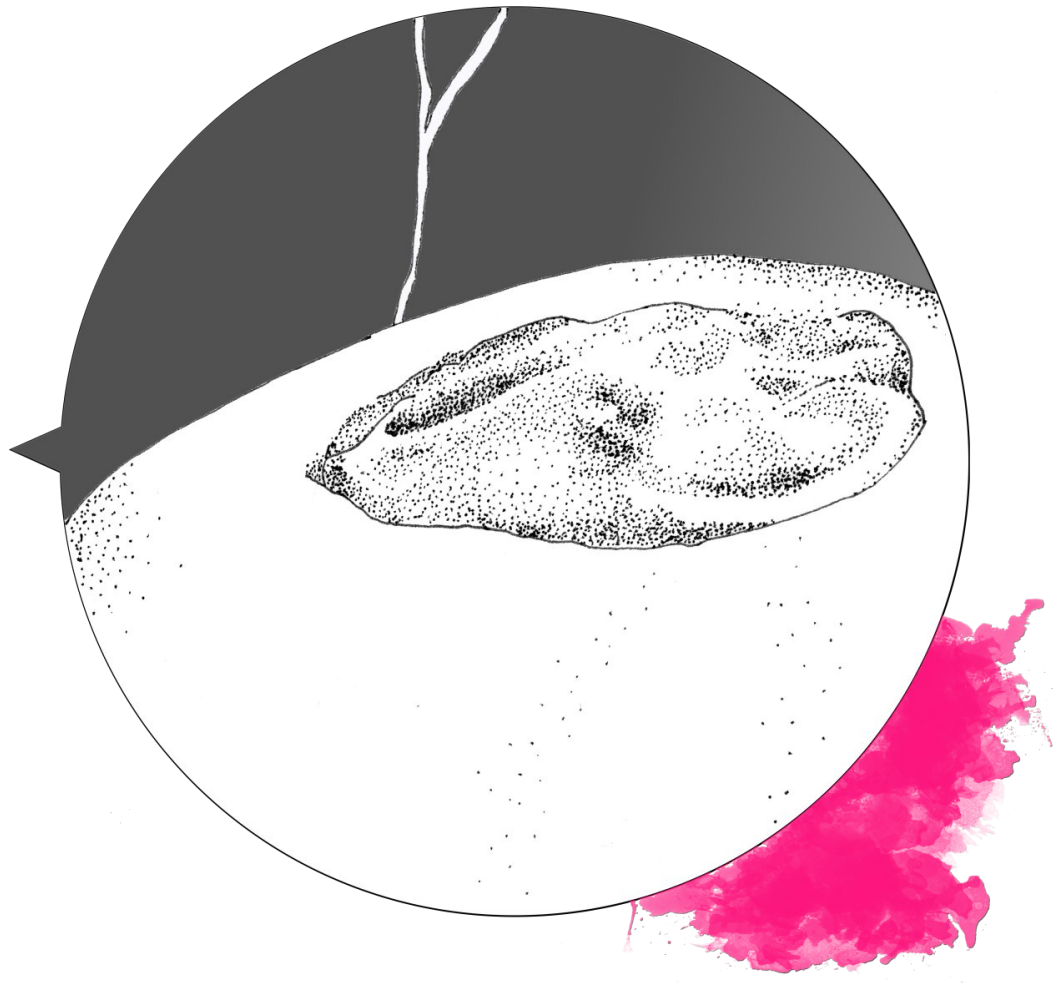




\section{Chapter}

\section{Lessons from a long-term equine study on cartilage repair with a co- culture of chondrons and MSCs in a fibre-reinforced gelatin methacryloyl hydrogel}

I.A.D. Mancini

J. Visser

P.H.Puhakka

M.H.P. van Rijen

F. Serra Bragança

H. Brommer

J. Töyräs

J. Malda

P.R. van Weeren 


\section{ABSTRACT}

Gelatin methacryloyl (GelMA) hydrogel has been described as a promising candidate to support the formation of a cartilage matrix by embedded cells in vitro. Reinforcement of the hydrogel with a structured microfiber scaffold has shown to significantly improve the biomechanical properties of the cell-laden structures. Despite promising in vitro results, the value of GelMA and reinforced GelMA for the repair of cartilage tissue in a large animal model remains to be demonstrated.

A surgical technique for implanting and UV-crosslinking (reinforced) GeIMA into full thickness chondral defects $(9 \mathrm{~mm}$ diameter) was developed in cadaveric equine stifle joints ( $n=3)$ and applied for a short-term implantation in one Shetland pony (two defects in one joint) to evaluate surgical feasibility and implant fixation. Next, a mixture of allogeneic chondrons and MSCs was implanted in (reinforced) GelMA hydrogels and in fibrin glue in both stifle joints of eight Shetland ponies and evaluated in a 12-month study. Images of the implants were obtained with arthroscopic optical coherence tomography after implantation, and at 2, 6 and 12 months. Kinetic and kinematic analyses were used to perform objective gait analysis before surgery, and at 2, 6 and 12 months.

Reinforced and non-reinforced GelMA could be successfully implanted in the equine model, and implants were still in place after 50 flexion-extension cycles in cadaveric joints. This was also the case in the pilot study, two weeks after implantation, and upon histological analysis, abundant infiltration of fibroblastic cells into the GelMA was observed. However, in the long-term study, optical coherence tomography indicated that reinforcements could not be detected anymore in defects after 2 months, and histological evaluation at 12 months suggested that early degradation of the hydrogel and insufficient fixation had led to fibrous repair of defects in all groups without any significant differences between the treatment groups. 


\section{INTRODUCTION}

Articular cartilage is a highly organized tissue characterized by its resilience against compressive and shear loading. Cartilage tissue accommodates and mitigates the forces generated by locomotion; its surface smoothness permits continuous motion between articulating parts of joints. It is also, however, a tissue known for its poor capacity to heal. Therefore, injuries may lead, in the long-term, to degeneration and development of osteoarthritis [1, 2]. Several solutions for the treatment of cartilage defects have been investigated over the years, leading to the development of a wide variety of repair techniques with variable clinical success [3-5]. Cell therapy has been explored since the 1990s [6] and autologous chondrocyte implantation (ACI) has been shown to provide long-term improvement in clinical trials [7]. However, the two-stage character of such procedures implies high costs and complex logistics associated with the necessary ex vivo expansion of cells, restricting their feasibility for large-scale application. Limiting the therapeutic approach to a single stage procedure could allow availability to a much wider public [8].

For the surgical delivery of cells, these need to be embedded in a cellfriendly delivery medium. In human orthopaedic medicine, fibrin glue is often used for this purpose, because of its easiness of surgical applicability and availability in clinical grade $[5,8]$. Nevertheless, fibrin glue has some significant limitations when used for regenerative medicine applications. The material cannot be functionalized, and once crosslinked, the polymers form a biomechanically weak network with a rapid degradation rate $[9,10]$. Many studies have therefore focused on developing instructive biomaterials such as hydrogels, with tuneable degradation rates that can be matched to tissue regeneration rates $[5,11]$. Among these, gelatin methacryloyl (GeIMA) has been identified as a potentially valid biomaterial for the engineering of cartilage tissue $[12,13]$.

GeIMA is a natural polymer derived from collagen type I [14], and crosslinkable by UV light with enzymatic or redox initiators [13]. Despite its tuneable characteristics, crosslinked GeIMA alone does not approximate the stiffness of native cartilage, and a reinforcement with a structure-giving material, such as polycaprolactone (PCL) may be required [15]. The reinforcing structure should still allow for sufficient elasticity and generation of mechanical stimuli that incite formation of the matrix that will support the 
embedded cells. Matching the mechanical properties of the native tissue is deemed to improve the performance of hydrogels when implanted [16], as cells in 3D matrices are known to respond to mechanical stimuli in terms of compression, tension and shear generated by loading [17].

Ultimate performance of biomaterials for cartilage repair is best evaluated in a long-term preclinical in vivo model $[18,19]$. The equine model is, although challenging, very relevant for the evaluation of potential therapies for cartilage repair, given the similarities between equine and human cartilage [20, 21]. In this case, the stifle joint of Shetland ponies was chosen as a model, for the reasons stated above, as well as for the sturdiness and aptness for group housing of these animals, which is preferable from an animal welfare viewpoint [22].

The aim of the research presented here was to evaluate GelMA's potential as a biomaterial for cartilage repair in a long-term in vivo equine model. A combination of allogeneic equine chondrons and mesenchymal stem cells (MSCs) that permitted carrying out the intervention as a one-step procedure was chosen for cell therapy, based on the promising results reported by a human clinical trial testing a combination of autologous chondrons and allogeneic MSCs [23, 24]. Several cell concentrations were tested and reinforced GelMA was compared with non-reinforced GelMA. Fibrin glue was used as a control. It was hypothesized that GelMA would outperform fibrin glue and that reinforced GelMA would perform substantially better than nonreinforced GeIMA.

\section{MATERIALS AND METHODS}

\section{Preparation of GelMA}

GelMA was synthesized by reaction of porcine type-A gelatin (Sigma-Aldrich, St. Louis, Missouri, USA) with methacrylic anhydride (Sigma-Aldrich) at $50^{\circ} \mathrm{C}$ for one hour, as previously described [14, 25]. Methacrylic anhydride was added dropwise to a $10 \%$ solution of gelatin in PBS under constant stirring, and to achieve a high degree of functionalization, $0.6 \mathrm{~g}$ of methacrylic anhydride per gram of gelatin were added. The functionalized polymer was then dialyzed against distilled water for 4 days at room temperature to remove methacrylic acid and anhydride and subsequently neutralized to $\mathrm{pH} 7.4$ with $10 \%$ sodium 
bicarbonate (Merck, Darmstadt, Germany).

For in vivo experiments, the dissolved GelMA was then filtered (SFCA membrane, $0.2 \mu \mathrm{m}$ pore size, Thermo Fisher Nalgene), prior to freeze-drying and stored at $-20^{\circ} \mathrm{C}$ until use, resulting in a degree of functionalization of circa $75 \%$ [25]. Upon use, defrosted GeIMA at a concentration of $10 \%(\mathrm{w} / \mathrm{v})$ was dissolved at $70^{\circ} \mathrm{C}$ in PBS containing photo-initiator Irgacure 2959 (Ciba, BASF, Ludwigshafen am Rhein, Germany) to obtain a final concentration of $0.1 \%$ (w/ v).

\section{Preparation of 3D printed microfiber scaffolds}

Scaffolds for in vivo reinforcement of GelMA were fabricated with a custommade melt electrospinning machine, using a direct-write mode, as previously described [26]. Fibres from polycaprolactone with a thickness of $14 \mu \mathrm{m}$ were stacked in a $0-90^{\circ}$ pattern to reach a height of $0.5 \mathrm{~mm}$, with a final mesh porosity of $92 \%$ (strand spacing $0.2 \mathrm{~mm}$ ) [15]. Scaffolds were cut to fit defect diameter $(9 \mathrm{~mm})$ and sterilized with two alternated washes in $70 \%$ ethanol and Milli-Q water.

\section{Harvest of equine chondrons and mesenchymal stromal cells}

Healthy cartilage was harvested under sterile conditions from the metatarsophalangeal joint of fresh equine cadavers ( $n=3$, age 3-10 years). After fragmentation and digestion in type II collagenase $(0.15 \% \mathrm{w} / \mathrm{v}$, Worthington Biochemical Corp, New Jersey, USA) at $37^{\circ} \mathrm{C}$ for 12 hours, the suspension was filtered through a $100 \mu \mathrm{m}$ cell strainer, washed in PBS and stored in liquid nitrogen at $-196^{\circ} \mathrm{C}$ in culture medium (Dulbecco's Modified Eagle Medium, DMEM, 41965, Invitrogen, California, USA) supplemented with $20 \%$ heatinactivated fetal bovine serum (FBS, BioWhittaker, USA) and 10\% dimethylsulfoxide (DMSO, Merck, Darmstadt, Germany).

With approval of the local animal ethical committee, a bone marrow aspirate from the sternum was obtained from a healthy, living equine donor $(n=1$, age 9 years). The mononuclear fraction (MNF) was isolated by centrifuging the sample on Ficoll-Paque (Sigma-Aldrich, Merck, Darmstadt, Germany). The MNF was seeded at a density of $2.5 \cdot 10^{5}$ cells $/ \mathrm{cm}^{2}$ and expanded in a monolayer culture till sub-confluency until passage 4 in MSC expansion medium containing a-MEM (22561, Invitrogen, California, USA) complemented with $10 \%$ heat-inactivated FBS, $0.2 \mathrm{mM}$ L-ascorbic acid 2-phosphate (Sigma-Aldrich, 
Merck, Darmstadt, Germany), 100 units $/ \mathrm{ml}$ penicillin and $100 \mu \mathrm{g} / \mathrm{ml}$

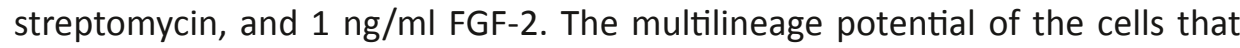
were cultured from the bone marrow aspirate was investigated by a three-way differentiation assay as previously described [27].

\section{UV-crosslinking and cell viability}

For in vivo cross-linking of GelMA in cartilage defects in ponies, a UV beam source was used with an intensity of $180 \mathrm{~mW} / \mathrm{cm}^{2}$ (350-450 nm, Hönle UV technology, Munich, Germany). Prior to the in vivo experiment, the effect of the duration of UV exposure on cell viability was evaluated.

Viability of the cell suspension acquired from cartilage was evaluated directly after digestion and after freeze/thaw, in order to check if chondrons can be frozen for allogeneic use. In addition, the viability of chondrons and MSCs from one donor, after several UV exposure times was assessed. A LIVE/DEAD Viability Assay (Molecular Probes MP03224, Eugene, USA) was performed according to the manufacturer's instructions. The samples were examined using an Olympus BX51 microscope and photomicrographs taken with an Olympus DP70 camera (Olympus, The Netherlands). The excitation/emission filters were set at $488 / 530 \mathrm{~nm}$ to observe living (green) cells and at 530/580 $\mathrm{nm}$ to detect dead (red) cells. Live and dead cells were counted in three gels or smears per time point, at a minimum of four locations. Viability was calculated as (alive cells / total cells counted) $\times 100 \%$.

\section{Measurement of stiffness}

Stiffness of GelMA constructs with embedded cells was measured after different crosslinking times. Samples ( $n=3$ for each crosslinking time) were subjected to single uniaxial unconfined compression in air at room temperature, after 1-hour submersion in PBS after preparation. Briefly, a force ramp (axial strain rate ca $20 \% / \mathrm{min}$ ) was applied to the samples for two minutes with a Dynamic Mechanical Analyser (DMA 2980, TA Instruments, New Castle, DE, USA). Stiffness was calculated from the linear derivative of the stress/strain curve between a strain of $10-15 \%$.

\section{Cadaveric studies for cartilage repair with GelMA}

The technique for making full thickness chondral defects in the medial trochlear ridge, and subsequent implantation and UV-crosslinking of 
(reinforced) GelMA was developed on three cadaveric equine stifle joints obtained from horses donated by their owners to science. The fixation of the implants was evaluated after layer by layer closure of the wound and subsequent manual execution of 50 flexion-extension cycles of the joint.

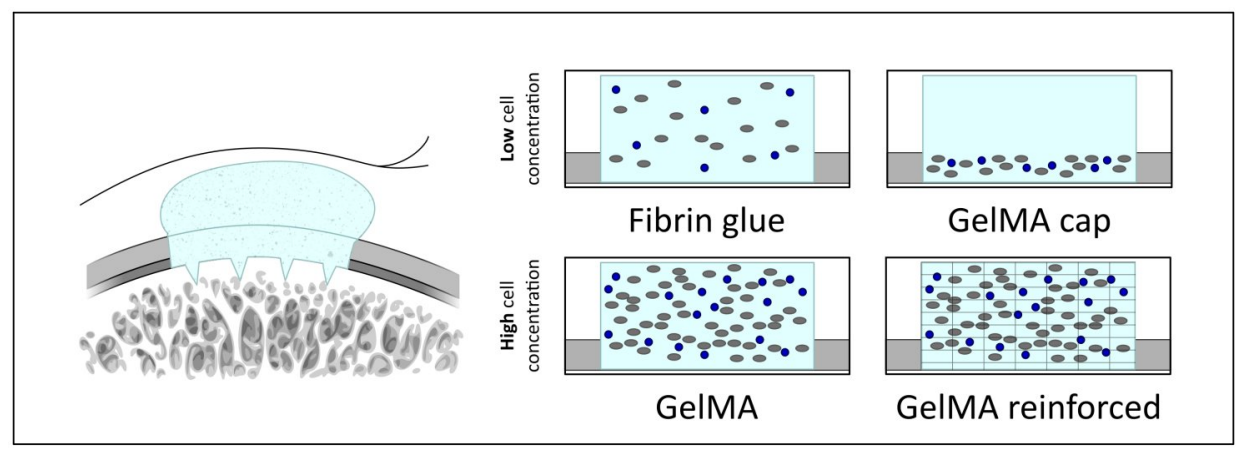

Figure 1. Experimental groups of in vivo long-term cartilage repair study. Two cartilage defects with a diameter of $9 \mathrm{~mm}$ were made in both stifle joints of Shetland ponies. The subchondral bone was microfractured for anchorage of the hydrogel. An allogeneic mixture of $80 \%$ MSCs (red) and 20\% chondrons (blue) was implanted in every defect, in low concentrations (Fibrin glue: 2 million cells/mL; GelMA low: 1 million cells on bottom of defect) and high cell concentrations (GelMA and reinforced GelMA: 20 million cells $/ \mathrm{mL}$ ).

\section{Pilot study for the cartilage repair model in a Shetland pony}

The animal studies were approved by the local Ethics Committee for Animal Experiments in compliance with the Institutional Guidelines on the Use of Laboratory Animals. The surgeries were performed at the Veterinary Faculty of Utrecht University. For evaluation of the surgical feasibility and hydrogel fixation, a pilot study was performed in one Shetland pony (age: 24 years, weight: $142 \mathrm{~kg}$ ). Two chondral defects were made in one stifle joint, and two protocols (experimental groups: reinforced GeIMA and GelMA cap) were implanted as described below. The pony was euthanized after two weeks (for veterinary education purposes) and the persistence of GelMA was assessed macroscopically and by histology and immunohistochemistry.

\section{Long-term evaluation of cartilage repair in Shetland ponies}

Four experimental conditions were tested in a long-term equine model, where GeIMA with a mixture of allogeneic MSCs and chondrons (80/20\% ratio) was cast into the full thickness cartilage defects, as illustrated in Figure 1. The four conditions included: 1) a homogeneously distributed low cell 
concentration ( 2 million cells $/ \mathrm{mL}$ ), comparable to the concentration currently used in human cartilage repair applied with fibrin glue. 2) A GelMA cap group in which a small volume of GeIMA in which 1 million cells were seeded was cast in the bottom part of the defect and subsequently covered with a layer of cell-free GeIMA. 3) A high cell concentration of 20 million cells $/ \mathrm{mL}$ homogeneously distributed in the GelMA hydrogel and 4) a similar high cell concentration in the GelMA hydrogel, this time reinforced with a 3D printed scaffold.

Two full thickness chondral defects were made in both stifle joints of eight Shetland ponies (age: $7 \pm 4$ years, weight $191 \pm 28 \mathrm{~kg}$, mean \pm SD). All 4 conditions were included in each animal, and low and high cell concentration groups were paired in every knee, with randomization of location within the stifle (proximal or distal) and side (left or right leg). Statistically, power was set at 0.90 , based on the ICRS macroscopic outcomes of a comparable study executed in goats by Bekkers et al. [28].

General anaesthesia was induced with midazolam and ketamine intravenously ( 0.06 and $2.2 \mathrm{mg} / \mathrm{kg}$, respectively), after premedication with detomidine and morphine $(10 \mathrm{mcg} / \mathrm{kg}$ and $0.1 \mathrm{mg} / \mathrm{kg}$, respectively). Anaesthesia was maintained with isoflurane (1.0-1.5\% end tidal in oxygen) with intravenous continuous rate infusion (CRI) of ketamine $(0.5 \mathrm{mg} / \mathrm{kg} / \mathrm{hr})$ and detomidine $(10 \mathrm{mcg} / \mathrm{kg} / \mathrm{hr}$ ). The medial trochlear ridge of the femur was exposed via a subpatellar approach to the femoropatellar joint, between the medial and middle patellar tendon. Two full-thickness defects of $9 \mathrm{~mm}$ in diameter were created in the cartilage (including calcified cartilage), by means of a hand-operated drill guided by a custom-made sleeve. Microfractures (10x, depth ca. $1 \mathrm{~mm}$ ) were subsequently made in the subchondral bone for improved fixation of the hydrogels.

A suspension of cells consisting of chondrons (p0) and MSCs (p4) (80 MSCs / $20 \%$ chondrons ratio) was centrifuged in the surgical theatre, in different quantities for each experimental group (Fig. 1); the cell pellet was then mixed in GelMA with a positive displacement pipet. The cell/hydrogel suspension was transferred into a syringe and a $21 \mathrm{G}$ needle (Becton, Dickenson and Company, New Jersey, USA) was mounted. A silicone-based sheet $(3 \times 3 \mathrm{~cm}$, Silon-SES, Bio Med Sciences, Allentown, USA, kindly provided by Regentis Biomaterials Ltd., Or-Akiva, Israel) was placed over the cartilage defect, where the sheet was fixated over the defect with a custom-made flexible fixation tip (diameter: 15 
$\mathrm{mm})$. The sheet was then punctured with the needle, and the hydrogel/cell suspension was infused in the defect. The hydrogel was crosslinked with a focused UV beam for 70 seconds, while contained in the defects by compression between the fixation tip onto the silicone sheet and the cartilage. In group 1 (Fig. 1), the cell pellet was suspended in the fibrinogen component, then mixed with the thrombin component and injected into the defect, where it was allowed to crosslink for 3 minutes. In group 2 (GelMA cap, Fig. 1), 1 million cells were infused in the bottom of the defect in $40 \mu \mathrm{l}$ GelMA and UVcrosslinked for 30 seconds, followed by a layer of cell-free GelMA sufficient to fill the defect, and subsequently UV-crosslinked for 60 seconds. In group 3, (GelMA high cell concentration, Fig. 1), 20 million cells/ml were infused in the defect encapsulated in the hydrogel. Finally, in group 4, (reinforced GelMA, Fig. 1), three microfiber scaffolds were stacked on top of each other in the cartilage defect prior to infusion of the hydrogel/cells mixture and subsequent crosslinking. UV-crosslinking of the hydrogel for groups 3 and 4 was 90 seconds.

The repaired defects with implanted hydrogels and surrounding cartilage were imaged with optical coherence tomography (OCT, Illumien Optis PCI Optimization System, St. Jude's Medical, St. Paul, MN, USA) along the length of three standardized paths over the implant, as described in Sarin et al. [29]. The wounds were closed in 4 layers using resorbable suture material (VicrylTM, Ethicon, US, LCC) and MonocryITM (Ethicon, US, LCC) for the skin. Stents were placed over the wound for protection for the first 48 hours and subsequently removed. Full weight bearing was allowed after recovery from anaesthesia. Post-operatively, the ponies received NSAIDS (meloxicam, $0.6 \mathrm{mg} / \mathrm{kg}, \mathrm{PO}$, BIDSID) up to 7 days and tramadol ( $5 \mathrm{mg} / \mathrm{kg}, P O, B I D-S I D)$ up to 3 days postoperatively. Prophylactically, antibiotics were administered perioperatively (ampicillin, 10-15 mg/kg, IV), while procaine penicillin $(20 \mathrm{mg} / \mathrm{kg}$, IM) was administered once after surgery.

Orthopaedic examination was performed prior to surgery to establish a base line, and repeated at 2, 6 and 12 months prior to arthroscopic assessment. Quantitative gait analysis was performed with the aid of kinematic and kinetic analysis, with data collection two weeks (T01) and one week (T02) before surgery, and at 2 (T1), 6 (T2), 9 (T3) and 12 (T4) months after lesion induction in both stifles. For kinematic analysis 6 infra-red cameras were used (ProReflex - Qualisys AB - Sweden) to track with motion capture the spherical reflective 
markers placed around both stifles during trot over the force plate. For kinetic analysis, ground reaction forces of both back limbs at trot were measured in all 3 axes using a stationary force plate (Z4852C - Kistler - Switzerland). Outcome parameters analysed were peak vertical force, stance duration and vertical impulse. Additionally, at 2, 6 and 12 months, tissue regeneration and biomaterial degradation was assessed arthroscopically: the surface was imaged with a standard arthroscopic camera, while the full depth of the repair tissue and underlying subchondral bone were imaged in detail with OCT (Illumien Optis PCI Optimization System, St. Jude's Medical, St. Paul, MN, USA).

At 12 months, animals were sedated with detomidine $(10 \mathrm{mcg} / \mathrm{kg})$, anesthetized with midazolam and ketamine intravenously $(0.06$ and $2.2 \mathrm{mg} / \mathrm{kg}$, respectively), and subsequently euthanized by administration of pentobarbital (50 mg/kg of body weight). Gross assessment of the medial trochlear ridge after euthanasia consisted of evaluating the volume of the repair tissue, integration of the margins with the surrounding native tissue and surface quality. After this, the entire osteochondral area of the medial femoral ridge containing the constructs was harvested for analysis using a surgical bone saw.

\section{Histology and immunohistochemistry}

The osteochondral tissue blocks containing the defects were fixated in 10\% formalin, decalcified with ethylene diamine tetra acetic acid (EDTA), then dehydrated through a graded ethanol series, cleared in xylene, and then embedded in paraffin. Samples were sectioned into $5 \mu \mathrm{m}$ slices and stained with haematoxylin and eosin (HE) and safranin-O. Repair was assessed by a blinded single observer using the modified O'Driscoll (MOD) score and the ICRS score [30], and cell infiltration evaluated using an Olympus BX51 light microscope.

Collagen types I and II were immunohistochemically stained after deparaffinization and rehydration of the sections. All sections were blocked in $5 \%$ bovine serum albumin and $0.3 \% \mathrm{H}_{2} \mathrm{O}_{2}$ following antigen retrieval. Rat collagen type I was retrieved by boiling the sections for 10 minutes in $10 \mathrm{mM}$ citrate buffer, $\mathrm{pH}$ 6. Antigen retrieval for collagen type II was performed by incubation with $1 \mathrm{mg} / \mathrm{mL}$ pronase (Sigma) and $10 \mathrm{mg} / \mathrm{mL}$ hyaluronidase (Sigma) at 37 degrees for half an hour each. Next, sections were incubated with the primary antibodies for collagen type I (1:250 Col1A1 Santa Cruz, Dallas, Texas, USA) collagen type II (1:100, monoclonal mouse, II-II6B3, DSHB) 
at $4^{\circ} \mathrm{C}$ overnight. Subsequently, collagen type I sections were treated with Polyclonal Goat Anti-Rabbit Immunoglobulins/HRP (1:200, Dako, Heverlee, Belgium) for 60 min at RT; collagen type II sections were incubated with GAMHRP (1:200, P0447, Dako) at room temperature for an hour. All collagen types were detected by a 10-minute conversion of 3,3'-diaminobenzidine solution (Sigma). Nuclei were counterstained with 50\% Mayer's haematoxylin. Isotype control stainings were carried out with either a murine IgG1 monoclonal antibody (Dako) at concentrations matching those used for the primary antibodies or by incubation without primary antibody.

\section{Statistical analysis}

Statistical analysis of histological scores was performed to detect differences between repair techniques with a one-way ANOVA; normality and homogeneity were assumed. When differences were detected, a Bonferroni post-hoc test was performed. A linear mixed model was used to compare the measured kinetic and kinematic parameters for each horse (random effect) between the different time points and between limbs (fixed effects). Significance was set at $5 \%$.

\section{RESULTS}

\section{The effect of UV-crosslinking on cell viability and hydrogel stiffness}

The stiffness of cell-laden GelMA gels increased with longer UV-exposure times (Fig. 2). The viability of embedded cells was between $79 \%$ and $93 \%$ up to 3 minutes of UV-exposure, but decreased to $0 \%$, when exposure was extended to 5 minutes (Fig. 2).

\section{Cadaver and pilot study in a Shetland pony}

The cadaveric studies showed that the infusion and UV-crosslinking of the GelMA hydrogel in cartilage defects was a technically feasible procedure. There was full penetration of the gel into the highly porous 3D-printed scaffolds. UV-crosslinking directly in the defect was successfully achieved on a cadaveric stifle joint. Crosslinked hydrogels were stable within the defect after the repetitive flexion/extension cycles.

Sterile intra-operative crosslinking of reinforced and non-reinforced GelMA hydrogels in chondral defects in the stifle joint of a Shetland pony proved to be 
feasible as well (Fig. 3A). The pony recovered well and GelMA hydrogel was still present in both cartilage defects two weeks after implantation, as observed macroscopically and confirmed histologically (Fig. 3B). The initial repair response showed infiltration of the biomaterial with cells of fibroblastic nature (Fig. 3B-3D).

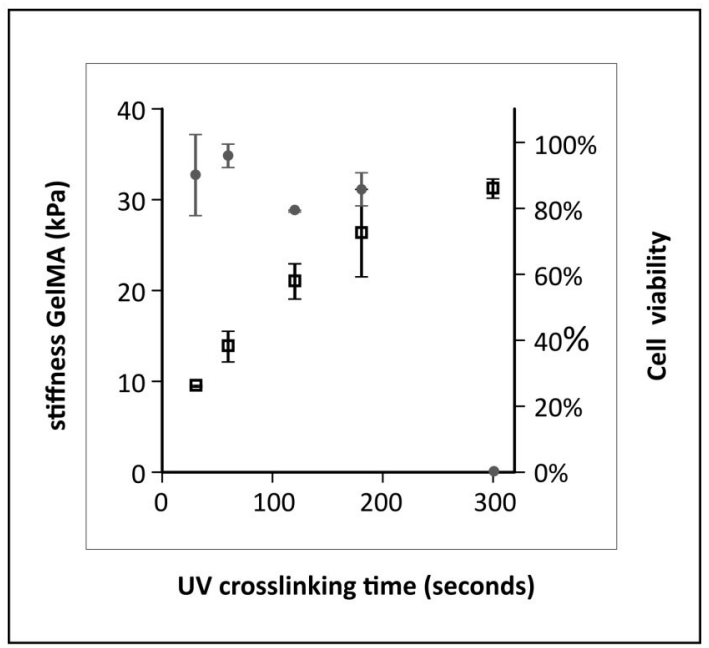

Figure 2. Cell viability and hydrogel stiffness depending on UV exposure times. The viability of chondrocytes and MSCs in GelMA decreased on UV exposure over 200 seconds (grey circles). The stiffness of hydrogels increased with longer UV exposure (black open squares). GelMA in the equine stifle joints was crosslinked for 70 seconds.

\section{Long-term evaluation of cartilage repair in Shetland ponies}

Imaging with OCT at initial surgery confirmed the depth of the defect, presence of microfractures, and the correct location of the implants (Fig. 4). All ponies walked clinically well after recovery from anaesthesia, with limited stiffness in the hind legs for the first few days after surgery. No signs of adverse reactions were detected post-operatively after first surgery and all animals healed following an expected recovery pattern. One pony had an adverse reaction to induction of anaesthesia at month 6 (2nd arthroscopy) and died; this animal was excluded from the study.

Gait analysis: Kinematic analysis detected no significant differences $(p>0.05)$ in the stifle range of motion (ROM) between time points and limbs. With the aid of kinetic analysis, it was observed that, taking the last measurement before surgery (T02) as a reference, there was a reduction in stance duration 

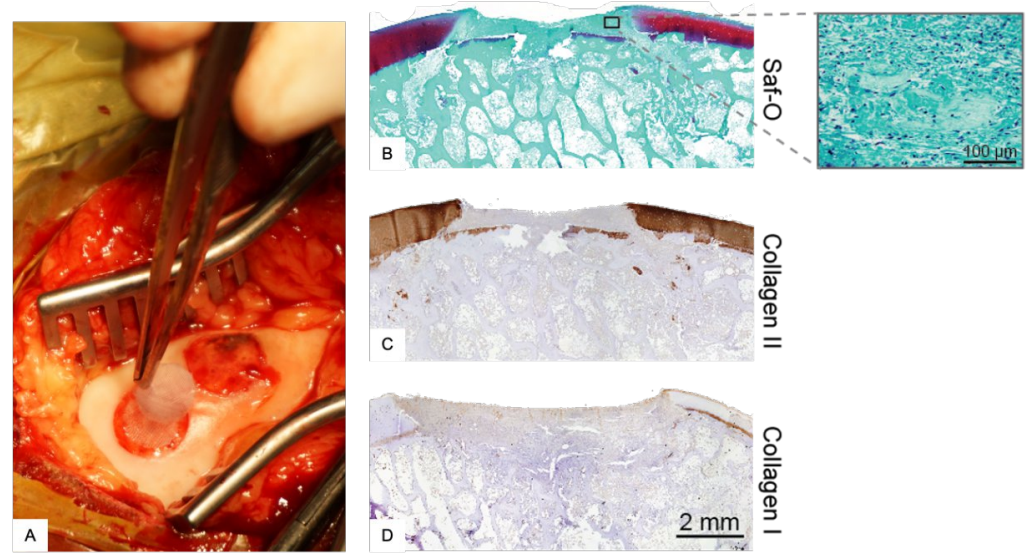

Figure 3. Pilot study in a Shetland pony for fixation of GelMA. A) specimens from the reinforced GelMA and the GelMA cap group were implanted in the stifle joint of one Shetland pony. B-D) Cells migrated into the hydrogel; only scarce remnants of the GelMA were observed in between the cells (light green in zoom picture).

at T1 $(p<0.001)$, an increase in stance duration at T3 $(p=0.03)$ (Fig. S1A), an increase in peak vertical force at T1 $(p<0.001)$ (Fig. S1B), and an increase in vertical impulse at T3 (Fig. S1C).

Arthroscopy: At 2 months post-operatively, defects appeared to be filled with a thin layer of repair tissue, and in many cases the underlying bone could be seen through the tissue, independently of repair group (Fig. 5, top row). Upon gentle probing with an arthroscopic hook probe, the tissue appeared subjectively soft and prone to fibrillation. At 6 and 12 months, generally the

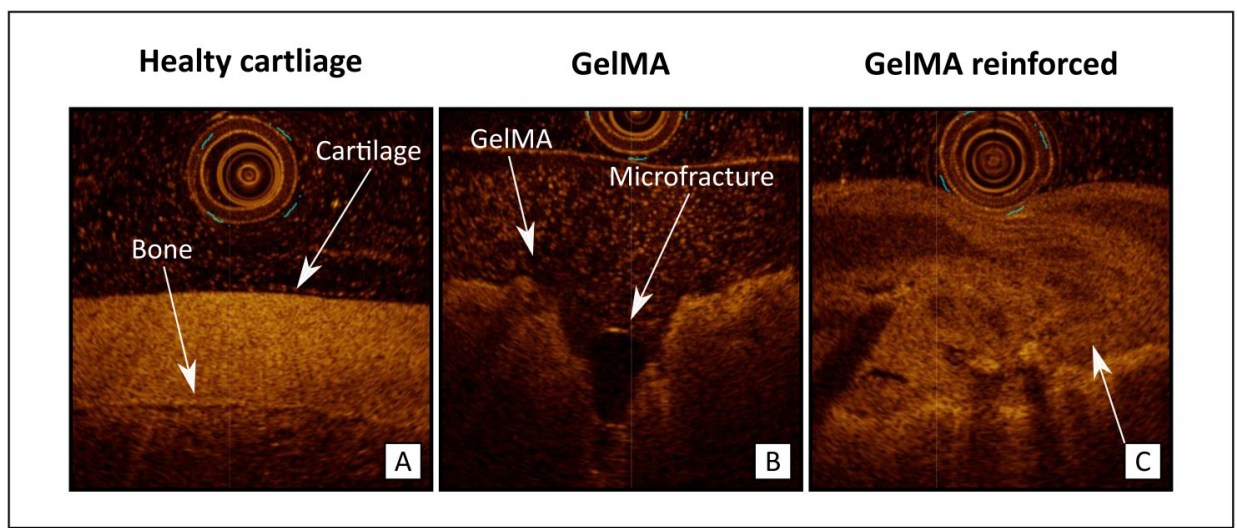

Figure 4. Baseline imaging of implants with OCT. A) Baseline values were obtained from healthy cartilage surrounding the defect directly after implantation. B) In the defect, the hydrogel and subchondral bone interface were easily identifiable. C) The reinforcing scaffold showed a pattern (white arrow) that allowed for assessment of correct placement. 


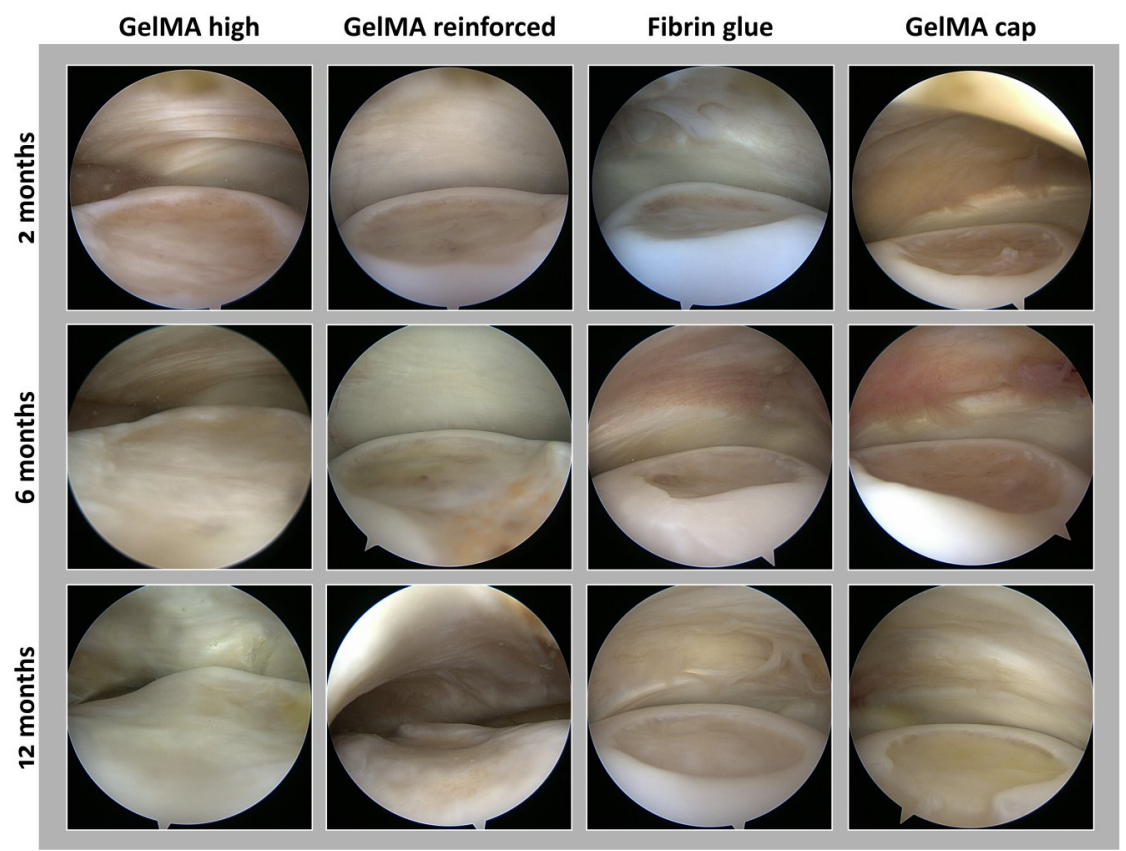

Figure 5. Arthroscopic imaging of defects. (Top row) 2 months post-operatively, defects are filled with a thin layer of repair tissue, with the underlying bone visible through the tissue, independently of repair group. (Middle row) 6 months after implantation, defects appear filled with more tissue. (Bottom row) 12 months post implantation defects are filled with tissue that is white and fibrous in appearance.

defects appeared filled with more tissue, white and fibrous in appearance, still soft upon probing and with a beginning of fibrillation (Fig. 5, bottom rows).

OCT: Right after implantation, at baseline, fibrin glue showed an inhomogeneous scattering signal (Fig. 6, top row). In the defects repaired with GelMA, the interface of repair tissue and surrounding cartilage was clearly visible (Fig. 4B, Fig, 6, top row, centre). The microfiber scaffolds were easily detected (Fig. 4C, Fig. 6, top row, right). After two months of healing, OCT imaging revealed repair tissue filling all defects; the thickness of the repair tissue on the subchondral bone was thin, between 100-300 $\mu \mathrm{m}$; in quality, and the tissue was optically similar to fibrous tissue (Fig. 6, second row). The reinforcing meshes were not detected anymore in any of the defects at the two-month time point. At 6 and 12 months, the findings were similar to those at two months (Fig. 6, last two rows). 


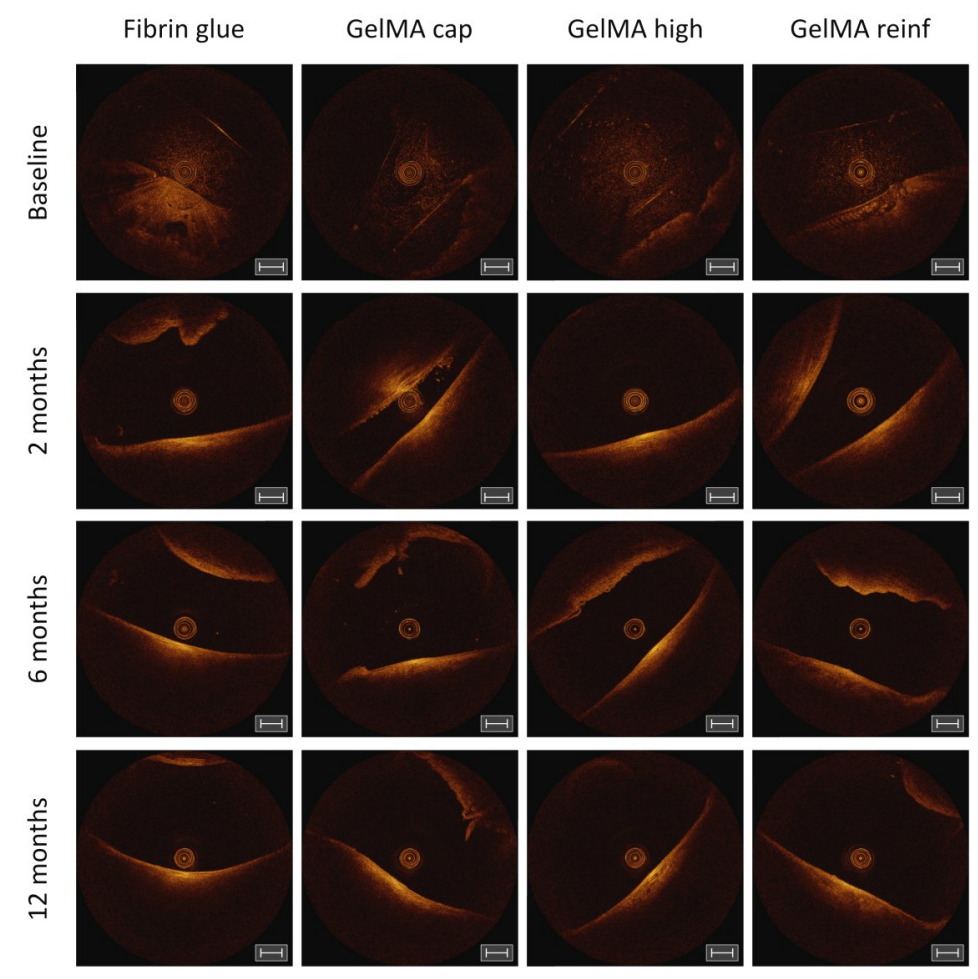

Figure 6. Optical coherence tomography imaging of defects. OCT imaging in vivo via an arthroscopic portal allowed real time monitoring of the evolution of repair tissue in the defects. The circle at the center represents the catheter that allows measurements, while the bright orange line on the lower halves of the windows (white arrows) represents the surface of the defects. After the initial time point (baseline), no clear differences in groups could be identified. Scale bar represents $1 \mathrm{~mm}$.

Histology: All defects were filled with a thin layer of repair tissue that appeared generally well integrated with the adjacent cartilage tissue. The repair tissue appeared of fibrous nature, with virtually no production of GAGs detected by safranin-O (Fig. 7); the subchondral bone directly under the defect appeared to be at a lower level than the subchondral bone underneath the surrounding native cartilage (Fig. 7). Histological evaluation using the ICRS score showed that fibrin glue outperformed other groups, however the MOD score was not different between the treatment groups (Fig. 8). 


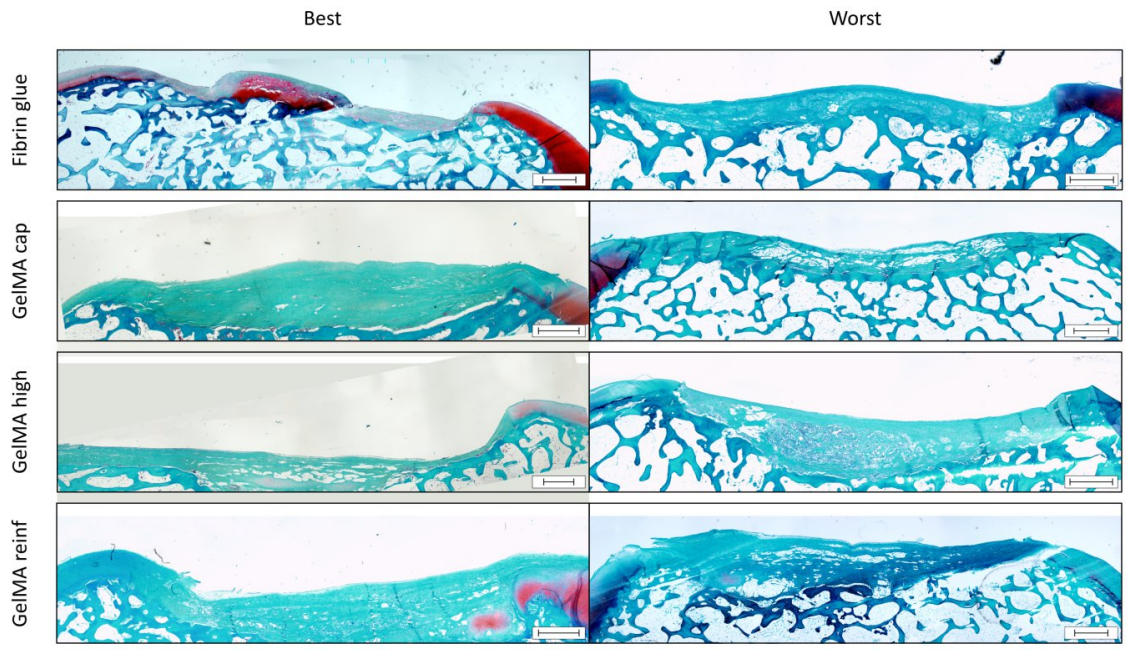

Figure 7. Histological sections of representative repaired defects. Safranin-O staining of representative samples for each group (fibrin glue, GeIMA cap, GeIMA high and GeIMA reinforced. Histology showed very scarce presence of glycosaminoglycans (red/pink) in the bestcase scenarios, whereas none can be detected in the worst cases, independently of repair strategy used. Scale bar is $1 \mathrm{~mm}$.

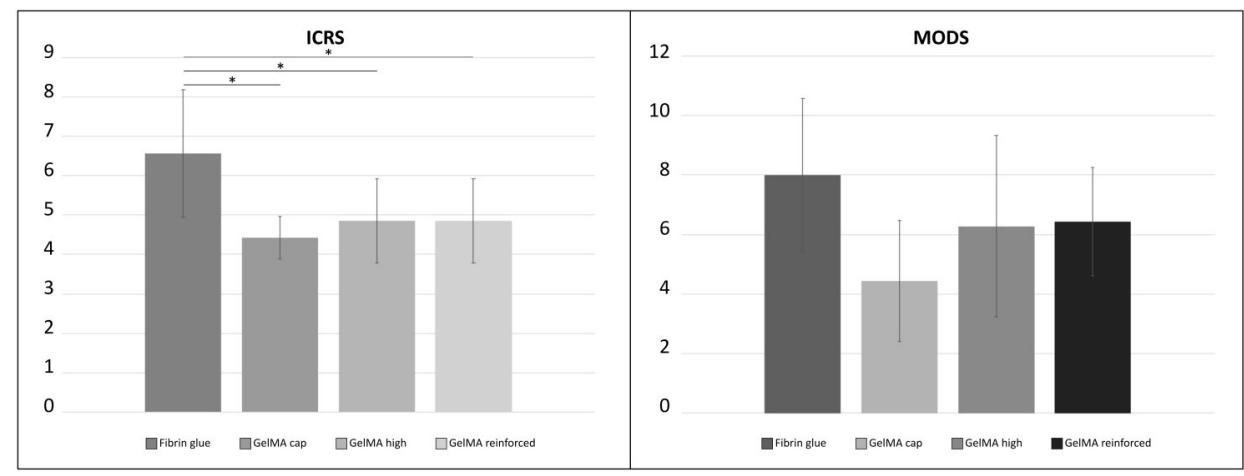

Figure 8. Histological scoring with ICRS and a modified O'Driscoll score system. Histological scoring with the ICRS system (left) showed that the control (fibrin glue) outperformed all other groups (* with $p<0.05)$. Histological scoring with MOD system (right) did not highlight differences between groups. 


\section{DISCUSSION}

The aim of this study was to evaluate the potential of the hydrogel GelMA as a biomaterial for cartilage repair in a long-term in vivo equine model. GeIMA was seeded with a mixture of allogeneic MSCs and chondrons in different concentrations, and in one group reinforced with a polycaprolactone (PCL) mesh. This in vivo study was based on the favourable results obtained with GelMA as a chondrogenic cell carrier in vitro [12, 13, 25], on the promising preliminary data from a human single-step procedure trial with a similar cell combination [23,31], and on the growing insight that reinforcement of inherently biomechanically weak hydrogels is necessary to generate a stable construct [15]. Moreover, the preliminary two-week pilot study further supported the potential value of a GelMA-based regeneration strategy. However, evaluation of the long-term performance in a sufficiently powered experimental study was still necessary.

Prior to clinical application, tissue-engineering strategies for cartilage repair need to be thoroughly tested, and notwithstanding the availability and usefulness of ex vivo platforms [32, 33], large animal studies are still deemed necessary for appropriate long-term evaluation. The equine model has emerged over the years as a suitable yet challenging model for this purpose: the cartilage of the stifle joint approximates that of the human knee both in thickness and composition, while simultaneously posing a challenging mechanical environment $[20,21]$. Additionally, the equine model represents a potential patient population on its own [34] and it offers the possibility to arthroscopically monitor the evolution of the repair sites. Controlled exercise regimens can be applied to evaluate responses to different rehabilitation protocols [21]. Principal limitations of the model are high associated costs and immediate load-bearing upon recovery from surgery [18]. Nevertheless, as demonstrated by Bolaños et al., while short-term results even in a preclinical model may be promising, longer periods of evaluation are required to assess the evolution of the repair tissue, in order to avoid overestimation of the performance of a repair strategy [18]. Ideally, cartilage reparative strategies should be evaluated over years rather than months, but there are practical and economical constraints to this approach in animal models. Therefore, the study reported here was purposely carried out over the period of 12 months.

Overall, this study's findings were not far from those observed in long-term 
repair of untreated defects, or after minimal interventions, such as microfracturing in humans [35] and large animal models [36]. Here, clinical outcome was satisfactory, and remained limited to post-surgical stiffness and a transient bilateral lameness.

However, results at tissue level were unsatisfactory and arthroscopy at 2 months showed a reduction of defect filling compared to the situation immediately post-surgery. Only a thin layer of repair tissue covered the bottom of the defect, with no apparent differences between treatments and there was no evidence of the remaining presence of the reinforcing meshes. This was strongly suggestive of all groups failing to elicit an appropriate regenerative response. Still, since the animals had shown sufficient clinical progress, and there was no apparent impact on their well-being, it was decided to carry the experiment to its original 12-month endpoint, provided no welfare issue would arise in the meantime. Indeed, quantitative gait analysis showed limited changes, characteristic of a mild and transient bilateral lameness, that was difficult to notice upon visual clinical examination. The only adverse event was the death upon induction of anaesthesia of one of the animals at the 6 months arthroscopy. The second-look arthroscopies performed at 6 and 12 months showed little change over time within the defects, with degeneration of the native tissue between the two defects. Histological data indeed confirmed failure to meet criteria for a functional repair, and the evaluation of the native tissue surrounding the defect showed progressive degeneration of cartilage [29] .

The discrepancy between the in vivo data and the in vitro results $[12,25]$ and possibly also the difference with the promising reports from the human clinical trial $[23,24]$ could be due to many factors, including the use of allogeneic chondrons, a higher degradation rate of the hydrogel and the resulting insufficient biomechanical resistance to the harsh biomechanical environment [37], the limited fixation of the reinforcing mesh and the immediate weight-bearing inherent to the equine model.

In the current study, chondrons and MSCs were combined to investigate their potential for cell-based cartilage repair. The combination of chondrons and MSCs has shown promise in human clinical studies on cartilage repair [24, 31]. However, the chondrons used in this study were of allogeneic origin, while autologous cells were used in the human trial. This choice was driven by the need of high amounts of cells for two of the experimental conditions, which 
could not be obtained via the rapid digestion protocol of autologous cartilage in a single-stage procedure [28]. This option was deemed acceptable as no adverse reactions had been reported in earlier use of allogeneic chondrocytes in stifle joints of horses [38]. It is, however, unclear whether allogeneic chondrons or chondrocytes hold a comparable regenerative potential as their autologous counterparts. The use of a combination of these cells with MSCs, is also supported by previous literature indicates that the proximity of chondrocytes to MSCs in GeIMA in vivo can prevent endochondral bone formation [39]. Moreover, while initially MSCs were thought to contribute directly to matrix formation, recent studies suggest that chondrocytes may be the primary contributors, leaving to MSCs a temporary trophic role [40, 41]. The absence of MSCs in biopsies of newly-formed cartilage a year after implantation with chondrons in the human clinical trial further supports this hypothesis [23].

It has been previously reported that the degradation rate of GeIMA in vitro and in vivo, in immunocompromised rats, was relatively slow [12, 39], making it reasonable to expect that the regenerative GelMA would provide a supporting matrix for the embedded cells for at least eight weeks. When implanted ectopically in rats, cell-free GelMA hydrogel constructs showed only limited cell infiltration. In contrast, when implanted for two weeks in the Shetland pony, the gel was strongly infiltrated by fibroblastic cells. This difference in cellular infiltration may be related to the abundant presence of cells from the bone marrow that can enzymatically break down the GeIMA, possibly accelerated by the immunocompetence of the animals. These dissimilarities due to species differences and implant location may have significantly speeded up the degradation process $[39,42]$. The difference in degradation between the two-week and two-month time points suggests that the duration of a pilot study should be in line with the expected degradation rate, as this is a key aspect in the early phases of preclinical translation of a cartilage repair strategy to large animal models [43].

Hydrogels have been designed to provide a cell-friendly, three-dimensional environment meant to mimic the extracellular matrix of native tissue, therefore, a biomaterial should enable cells to produce a matrix with specific characteristics [44]. However, while providing the necessary water-rich environment, hydrogels often have inadequate mechanical properties, resulting in a biomaterial too soft for application, particularly for the 
biomechanical challenges of cartilage repair $[13,26]$. Although the mechanical properties of hydrogels can be improved by modifying polymer concentration or crosslink density, such modifications often compromise the biological performance of the biomaterial $[45,46]$. To address this limitation, a strategy using a composite reinforced hydrogel was included in the study design. The addition of a PCL scaffold with micrometre-scale fibres, organized and interconnected via melt-electrospinning writing, allowed to fabricate a highstrength construct with customizable mechanical properties that still supported cell proliferation and extracellular matrix production in vitro [15].

The constructs, reinforced or not, along with a control group composed of the same cell mixture suspended in fibrin glue, were directly cast to repair fullthickness chondral defects at the bottom of which microfractures were created in the subchondral bone to improve penetration of the hydrogel. Other than the fixation provided by the direct casting and the hydrogel penetration into the microfractures, no additional fixation techniques were applied in this study. Currently, there are no well-established techniques for fixating chondral scaffolds in the horse. In humans, the direct casting of fibrin glue is considered a viable option [47]. However, because of the immediate post-surgical weight-bearing in the equine model, this solution may not be equally valid [21], apart from the inaptness of the commercially used products for use in the horse [48].

The reinforcing fibre-meshes were shown to have been correctly placed by OCT after implantation. In addition, the mesh used in the pilot study was still in place two weeks after implantation. However, after two months the PCL mesh could not be traced. This absence was confirmed at later time points, and ultimately upon explantation. A possible explanation for this could be the rapid degradation of the hydrogel, leaving the fibre scaffold exposed and prone to being pushed out of the defect during locomotion. The alternative possibility of the early degradation of the PCL-based microfibers of the reinforcement is far less likely given the well-characterized material properties of PCL, however, it cannot be completely excluded. The likely early disappearance of the gel, be it due to early degeneration or because of direct mechanical impact, may also be the main explanatory factor in the lack of any difference in performance between the implants with high and low cell density.

The study presented here offers insight into common issues that may occur 
when translating repair strategies for cartilage repair to a large animal model, even with the unsatisfactory outcome. The results clearly underscore the need for long-term assessment of repair strategies in large animal models, as advocated earlier [18]. Importantly, it also highlights how relevant matters such as fixation of biomaterials or their degradation rates are for the success of cartilage repair strategies. Future research should, therefore, focus on combining fixation without disruption of the biomaterial with a degradation rate further tuned to the rate of matrix production.

\section{ACKNOWLEDGMENTS}

The authors would like to thank J.C. de Grauw and J.P.A.M. van Loon and their team for taking care of anaesthesia of the ponies and the complete 'equine cartilage repair team' for logistics around the surgeries.

The research leading to these results has received funding from the European Community's Seventh Framework Programme (FP7/2007- 2013) under grant agreement 309962 (HydroZONES), the Dutch Arthritis Association (ReumaNL, LLP-12 and LLP-22), the Academy of Finland (decision number: 267551) and Kuopio University Hospital (VTR funding). 


\section{REFERENCES}

[1] Z. Yang, Y. Shi, X. Wei, J. He, S. Yang, G. Dickson, J. Tang, J. Xiang, C. Song, G. Li, Fabrication and repair of cartilage defects with a novel acellular cartilage matrix scaffold, Tissue Engineering Part C: Methods 16(5) (2009) 865-876.

[2] A.F. Steinert, S.C. Ghivizzani, A. Rethwilm, R.S. Tuan, C.H. Evans, U. Nöth, Major biological obstacles for persistent cell-based regeneration of articular cartilage, Arthritis research \& therapy 9(3) (2007) 213.

[3] J.S. Temenoff, A.G. Mikos, Review: tissue engineering for regeneration of articular cartilage, Biomaterials 21(5) (2000) 431-440.

[4] G. Smith, G. Knutsen, J. Richardson, A clinical review of cartilage repair techniques, Bone \& Joint Journal 87(4) (2005) 445-449.

[5] E.A. Makris, A.H. Gomoll, K.N. Malizos, J.C. Hu, K.A. Athanasiou, Repair and tissue engineering techniques for articular cartilage, Nature Reviews Rheumatology 11(1) (2015) 21.

[6] M. Brittberg, A. Lindahl, A. Nilsson, C. Ohlsson, O. Isaksson, L. Peterson, Treatment of deep cartilage defects in the knee with autologous chondrocyte transplantation, New england journal of medicine 331(14) (1994) 889-895.

[7] L. Peterson, H.S. Vasiliadis, M. Brittberg, A. Lindahl, Autologous chondrocyte implantation: a long-term follow-up, The American journal of sports medicine 38(6) (2010) 1117-1124.

[8] S.C. Mastbergen, D.B. Saris, F.P. Lafeber, Functional articular cartilage repair: here, near, or is the best approach not yet clear?, Nature reviews rheumatology 9(5) (2013) 277.

[9] D. Deponti, A. Di Giancamillo, L. Mangiavini, A. Pozzi, G. Fraschini, C. Sosio, C. Domeneghini, G.M. Peretti, Fibrin-based model for cartilage regeneration: tissue maturation from in vitro to in vivo, Tissue Engineering Part A 18(11-12) (2012) 1109-1122.

[10] G.N. Homminga, P. Buma, H.W. Koot, P.M. van der Kraan, W.B. van den Berg, Chondrocyte behavior in fibrin glue in vitro, Acta Orthopaedica Scandinavica 64(4) (1993) 441-445.

[11] K.L. Spiller, S.A. Maher, A.M. Lowman, Hydrogels for the repair of articular cartilage defects, Tissue engineering part B: reviews 17(4) (2011) 281-299.

[12] P.A. Levett, F.P. Melchels, K. Schrobback, D.W. Hutmacher, J. Malda, T.J. Klein, A biomimetic extracellular matrix for cartilage tissue engineering centered on photocurable gelatin, hyaluronic acid and chondroitin sulfate, Acta biomaterialia 10(1) (2014) 214-223.

[13] B.J. Klotz, D. Gawlitta, A.J. Rosenberg, J. Malda, F.P. Melchels, Gelatin-methacryloyl hydrogels: towards biofabrication-based tissue repair, Trends in biotechnology 34(5) (2016) 394407.

[14] A.I. Van Den Bulcke, B. Bogdanov, N. De Rooze, E.H. Schacht, M. Cornelissen, H. Berghmans, Structural and rheological properties of methacrylamide modified gelatin hydrogels, Biomacromolecules 1(1) (2000) 31-38.

[15] J. Visser, F.P. Melchels, J.E. Jeon, E.M. Van Bussel, L.S. Kimpton, H.M. Byrne, W.J. Dhert, P.D. Dalton, D.W. Hutmacher, J. Malda, Reinforcement of hydrogels using three-dimensionally printed microfibres, Nature communications 6 (2015) 6933.

[16] F.T. Moutos, L.E. Freed, F. Guilak, A biomimetic three-dimensional woven composite scaffold for functional tissue engineering of cartilage, Nature materials 6(2) (2007) 162.

[17] D.E. Discher, P. Janmey, Y.-I. Wang, Tissue cells feel and respond to the stiffness of their substrate, Science 310(5751) (2005) 1139-1143.

[18] R.V. Bolaños, S. Cokelaere, J.E. McDermott, K. Benders, U. Gbureck, S. Plomp, H. Weinans, J. Groll, P. van Weeren, J. Malda, The use of a cartilage decellularized matrix scaffold for the repair of osteochondral defects: the importance of long-term studies in a large animal model, Osteoarthritis and Cartilage 25(3) (2017) 413-420.

[19] M.B. Hurtig, M.D. Buschmann, L.A. Fortier, C.D. Hoemann, E.B. Hunziker, J.S. Jurvelin, P. Mainil-Varlet, C.W. Mcllwraith, R.L. Sah, R.A. Whiteside, Preclinical studies for cartilage repair: recommendations from the International Cartilage Repair Society, Cartilage 2(2) (2011) 137-152. [20] J. Malda, K. Benders, T. Klein, J. De Grauw, M. Kik, D. Hutmacher, D. Saris, P. Van Weeren, W. Dhert, Comparative study of depth-dependent characteristics of equine and human 
osteochondral tissue from the medial and lateral femoral condyles, Osteoarthritis and cartilage 20(10) (2012) 1147-1151.

[21] C.W. Mcllwraith, L.A. Fortier, D.D. Frisbie, A.J. Nixon, Equine models of articular cartilage repair, Cartilage 2(4) (2011) 317-326.

[22] K. Yarnell, C. Hall, C. Royle, S.L. Walker, Domesticated horses differ in their behavioural and physiological responses to isolated and group housing, Physiology \& behavior 143 (2015) 51-57.

[23] T.S. de Windt, L.A. Vonk, I. Slaper-Cortenbach, M.P. van den Broek, R. Nizak, M.H. van Rijen, R.A. de Weger, W.J. Dhert, D.B. Saris, Allogeneic mesenchymal stem cells stimulate cartilage regeneration and are safe for single-stage cartilage repair in humans upon mixture with recycled autologous chondrons, Stem Cells 35(1) (2017) 256-264.

[24] D.D. Frisbie, H.E. McCarthy, C.W. Archer, M.F. Barrett, C.W. Mcllwraith, Evaluation of articular cartilage progenitor cells for the repair of articular defects in an equine model, JBJS 97(6) (2015) 484-493.

[25] W. Schuurman, P.A. Levett, M.W. Pot, P.R. van Weeren, W.J. Dhert, D.W. Hutmacher, F.P. Melchels, T.J. Klein, J. Malda, Gelatin-methacrylamide hydrogels as potential biomaterials for fabrication of tissue-engineered cartilage constructs, Macromolecular bioscience 13(5) (2013) 551-561.

[26] K.W. Boere, J. Visser, H. Seyednejad, S. Rahimian, D. Gawlitta, M.J. Van Steenbergen, W.J. Dhert, W.E. Hennink, T. Vermonden, J. Malda, Covalent attachment of a three-dimensionally printed thermoplast to a gelatin hydrogel for mechanically enhanced cartilage constructs, Acta biomaterialia 10(6) (2014) 2602-2611.

[27] D. Gawlitta, E. Farrell, J. Malda, L.B. Creemers, J. Alblas, W.J. Dhert, Modulating endochondral ossification of multipotent stromal cells for bone regeneration, Tissue engineering. Part B, Reviews 16(4) (2010) 385-95.

[28] J.E. Bekkers, A.I. Tsuchida, M.H. van Rijen, L.A. Vonk, W.J. Dhert, L.B. Creemers, D.B. Saris, Single-stage cell-based cartilage regeneration using a combination of chondrons and mesenchymal stromal cells: comparison with microfracture, The American journal of sports medicine 41(9) (2013) 2158-2166.

[29] J.K. Sarin, N.C. Te Moller, I.A. Mancini, H. Brommer, J. Visser, J. Malda, P.R. van Weeren, I.O. Afara, J. Töyräs, Arthroscopic near infrared spectroscopy enables simultaneous quantitative evaluation of articular cartilage and subchondral bone in vivo, Scientific reports 8(1) (2018) 13409.

[30] P. Mainil-Varlet, B. Van Damme, D. Nesic, G. Knutsen, R. Kandel, S. Roberts, A new histology scoring system for the assessment of the quality of human cartilage repair: ICRS II, The American journal of sports medicine 38(5) (2010) 880-890.

[31] T. de Windt, L.A. Vonk, I.C. Slaper-Cortenbach, M.H. van Rijen, W.J. Dhert, D.B.F. Saris, Allogeneic mesenchymal stem cells are safe and stimulate cartilage regeneration upon coimplantation with chondrons in the IMPACT trial, Abstract no. 16.3.2 at the International Cartilage Repair Society conference, Chicago (2015).

[32] A. Schwab, A. Meeuwsen, F. Ehlicke, J. Hansmann, L. Mulder, A. Smits, H. Walles, L. Kock, Ex vivo culture platform for assessment of cartilage repair treatment strategies, ALTEX-Alternatives to animal experimentation 34(2) (2017) 267-277.

[33] R. LeBaron, K. Athanasiou, Ex vivo synthesis of articular cartilage, Biomaterials 21(24) (2000) 2575-2587.

[34] R. Williams, L. Harkins, C. Hammond, J. Wood, Racehorse injuries, clinical problems and fatalities recorded on British racecourses from flat racing and National Hunt racing during 1996, 1997 and 1998, Equine veterinary journal 33(5) (2001) 478-486.

[35] R.F. LaPrade, L.S. Bursch, E.J. Olson, V. Havlas, C.S. Carlson, Histologic and immunohistochemical characteristics of failed articular cartilage resurfacing procedures for osteochondritis of the knee: a case series, The American journal of sports medicine 36(2) (2008) 360-368.

[36] E. Salonius, L. Rieppo, M.J. Nissi, H.J. Pulkkinen, H. Brommer, A. Brünott, T.S. Silvast, P.R. Van Weeren, V. Muhonen, P.A. Brama, Critical-sized cartilage defects in the equine carpus, Connective Tissue Research 60(2) (2019) 95-106. 
[37] C.J. Moran, A. Ramesh, P.A. Brama, J.M. O'Byrne, F.J. O'Brien, T.J. Levingstone, The benefits and limitations of animal models for translational research in cartilage repair, Journal of experimental orthopaedics 3(1) (2016) 1.

[38] K. Ortved, A. Nixon, H. Mohammed, L. Fortier, Treatment of subchondral cystic lesions of the medial femoral condyle of mature horses with growth factor enhanced chondrocyte grafts: a retrospective study of 49 cases, Equine veterinary journal 44(5) (2012) 606-613.

[39] J. Visser, D. Gawlitta, K.E. Benders, S.M. Toma, B. Pouran, P.R. van Weeren, W.J. Dhert, J. Malda, Endochondral bone formation in gelatin methacrylamide hydrogel with embedded cartilage-derived matrix particles, Biomaterials 37 (2015) 174-182.

[40] T.S. de Windt, J.A. Hendriks, X. Zhao, L.A. Vonk, L.B. Creemers, W.J. Dhert, M.A. Randolph, D.B. Saris, Concise review: unraveling stem cell cocultures in regenerative medicine: which cell interactions steer cartilage regeneration and how?, Stem cells translational medicine 3(6) (2014) 723-733.

[41] L. Wu, H.-J. Prins, M.N. Helder, C.A. van Blitterswijk, M. Karperien, Trophic effects of mesenchymal stem cells in chondrocyte co-cultures are independent of culture conditions and cell sources, Tissue Engineering Part A 18(15-16) (2012) 1542-1551.

[42] J.W. Nichol, S.T. Koshy, H. Bae, C.M. Hwang, S. Yamanlar, A. Khademhosseini, Cell-laden microengineered gelatin methacrylate hydrogels, Biomaterials 31(21) (2010) 5536-5544.

[43] D.J. Huey, J.C. Hu, K.A. Athanasiou, Unlike bone, cartilage regeneration remains elusive, Science 338(6109) (2012) 917-921.

[44] N.C. Hunt, L.M. Grover, Cell encapsulation using biopolymer gels for regenerative medicine, Biotechnology letters 32(6) (2010) 733-742.

[45] J. Malda, J. Visser, F.P. Melchels, T. Jüngst, W.E. Hennink, W.J. Dhert, J. Groll, D.W. Hutmacher, 25th anniversary article: engineering hydrogels for biofabrication, Advanced materials 25(36) (2013) 5011-5028.

[46] C.A. DeForest, K.S. Anseth, Advances in bioactive hydrogels to probe and direct cell fate, Annual review of chemical and biomolecular engineering 3 (2012) 421-444.

[47] J. Bekkers, A. Tsuchida, J. Malda, L. Creemers, R. Castelein, D.B. Saris, W. Dhert, Quality of scaffold fixation in a human cadaver knee model, Osteoarthritis and cartilage 18(2) (2010) 266272.

[48] I.A. Mancini, R.A. Vindas Bolaños, H. Brommer, M. Castilho, A. Ribeiro, J.P. Van Loon, A. Mensinga, M.H. Van Rijen, J. Malda, R. van Weeren, Fixation of hydrogel constructs for cartilage repair in the equine model: a challenging issue, Tissue Engineering Part C: Methods 23(11) (2017) 804-814. 


\section{SUPPLEMENTAL DATA}
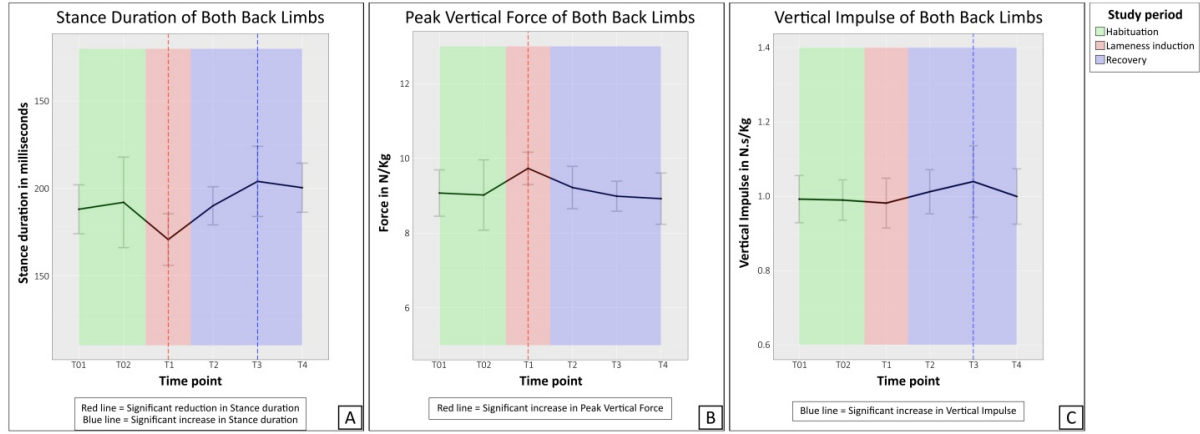

Figure S1. Objective gait analysis with force plate. (A) Stance duration was significantly reduced at T1 $(p<0.001)$, and significantly increased at T3 $(p=0.032)$. (B) Peak vertical force was significantly increased at T1 $(p<0.001)$. (C) Vertical impulse was significantly increased at T3. No significant ( $p>0.05)$ differences in speed or between limbs were observed between the different time points except for vertical impulse where a significant difference between limbs was found $(p=0.007)$. 


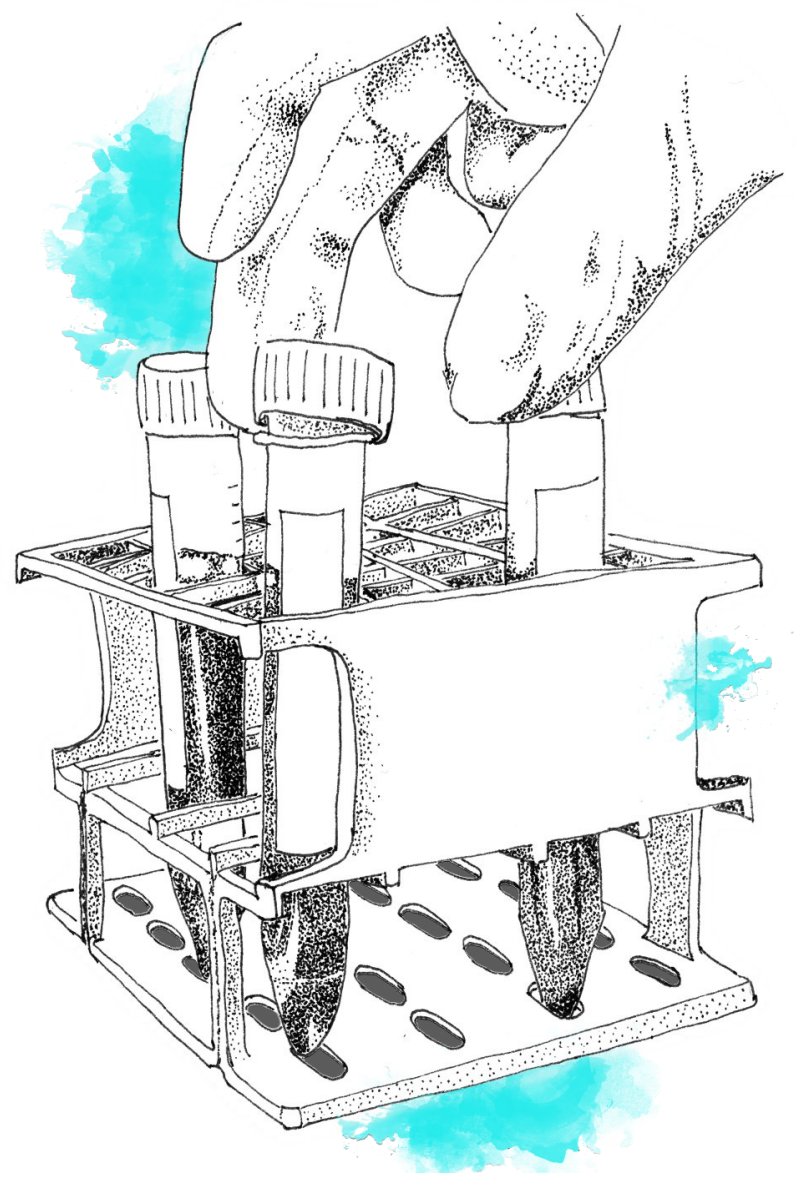




\title{
Chapter
}

\section{Fixation of hydrogel constructs for cartilage repair in the equine model: a challenging issue}

\author{
I.A.D. Mancini \\ R.A. Vindas Bolaños \\ H. Brommer \\ M. Castilho \\ A. Ribeiro \\ J.P.A.M. van Loon \\ A. Mensinga \\ M.H.P. van Rijen \\ J. Malda \\ P.R. van Weeren
}

Tissue Engineering Part C, 2017, 23(11):804-814 


\section{ABSTRACT}

Objective: To report on the experiences with the use of commercial and autologous fibrin glue (AFG) and of an alternative method based on a 3Dprinted polycaprolactone ( $\mathrm{PCL}$ ) anchor for the fixation of hydrogel-based scaffolds in an equine model for cartilage repair.

Methods: In a first study, three different hydrogel-based materials were orthotopically implanted in nine horses for 1-4 weeks in $6 \mathrm{~mm}$ diameter full thickness cartilage defects in the medial femoral trochlear ridge and fixated with commercially available fibrin glue (CFG). One defect was filled with CFG only as a control.

In a second study, CFG and AFG were compared in an ectopic equine model. The third study compared the efficacy of AFG and a 3D-printed PCL-based osteal anchor for fixation of PCL-reinforced hydrogels in three horses for 2 weeks, with a 4 week follow-up to evaluate integration of bone with the PCL anchor. Short-term scaffold integration and cell infiltration were evaluated by microcomputed tomography and histology as outcome parameters.

Results: The first study showed signs of subchondral bone resorption in all defects, including the controls filled with CFG only, with significant infiltration of neutrophils. Ectopically, CFG induced clear inflammation with strong neutrophil accumulation; AFG was less reactive, showing fibroblast infiltration only.

In the third study the fixation potential for PCL-reinforced hydrogels of AFG was inferior to the PCL anchor. PCL reinforcement had disappeared from two defects and showed signs of dislodging in the remaining four. All six constructs fixated with the PCL anchor were still in place after 2 weeks. At 4 weeks, the $\mathrm{PCL}$ anchor showed good integration and signs of new bone formation.

Conclusions: The use of AFG should be preferred to xenogeneic products in the horse, but AFG is subject to individual variations and laborious to make. The PCL anchor provides the best fixation; however, this technique involves the whole osteochondral unit, which entails a different conceptual approach to cartilage repair. 


\section{INTRODUCTION}

Articular cartilage is a highly specialized tissue in diarthrodial joints. Its roles are to provide smooth motion between joint surfaces, to transmit the forces generated by locomotion and to attenuate these by redistributing mechanical stress to the underlying bone. However, because of its avascular nature, cartilage has poor intrinsic capacities for self-repair [1]. Cartilage injuries in young adults due to sports injuries may, therefore, lead in the long term to degeneration of the tissue; this is one of the causes of osteoarthritis, which is a major cause of disability among the elder $[2,3]$.

Many potential solutions for the treatment of cartilage defects have been investigated, leading to a wide variety of repair techniques being proposed over the years [4-6]. However, despite the researchers' efforts, no method for true regeneration of tissue has been found yet, and the quest for the successful induction of native-like hyaline cartilage is still ongoing [7]. In this quest, tissue engineering is a promising and appealing approach.

Tissue engineering combines cells, scaffolds and bioactive molecules to support, guide and maintain the restoration of native tissue through controlled degradation rates that should balance with the process of tissue regeneration [8]. In particular, researchers have focused on providing an appropriate degradable matrix for cells to survive and differentiate. Hydrogels have shown to be suitable biomaterials for this purpose because of their intrinsic hydrated nature, their capacity to incorporate chemical cues, and their potential biocompatibility $[8,9]$. Before possible clinical application, in vitro tissueengineered constructs need to be tested and, despite growing availability of in silico [10] and ex vivo $[11,12]$ models, large animal models are still deemed essential as final proof of concept. In the case of orthopaedic disorders in general and cartilage repair in particular, the equine model has been often described as a very suitable model, as the articular cartilage in the equine stifle joint closely resembles that in the human knee with respect to thickness and biochemical composition, and both species present a very challenging mechanical environment $[13,14]$.

A crucial issue in cartilage repair is the fixation of implants. A range of approaches has been reported in literature, but all have significant drawbacks. For example, fascial/periosteal flaps sutured over the defect cause osteoarthritis-like changes in adjacent cartilage [15], and the use of 
transosseous sutures or biodegradable pins alters the architecture of 3D scaffolds [16]. While it is possible to cast directly materials into defects, or to use hydrogels as a glue [17], these techniques are laborious, difficult for translation to human clinics, and limit greatly the possibility to control the design of the implants. Fibrin glue has been extensively described in literature as a fixation tool for various type of scaffolds for cartilage repair in animal models [18, 19], and is currently used in human clinics [20]. It has the advantage that it does not physically alter either the scaffold or the tissues adjacent to the defect; however, its use is not uncontested. First, there may be a species issue. Brehm described how the use of fibrin glue of human origin caused a massive cell infiltration in the subchondral bone of goats [21]. Although thus far not reported in literature, a similar situation may exist in the horse. However, problems have been reported in studies where equine fibrin is used as a vehicle for cellular therapies in the horse (Mcllwraith, pers. comm. 2016), including demonstration of the lack of ability for mesenchymal stem cells to migrate and proliferate in full strength fibrin and the requirement of dilution of the fibrin to allow these processes [22] and that the addition of MSCs to autologous platelet-enhanced fibrin scaffolds in chondral defects had inferior results to the platelet-enhanced fibrin scaffolds alone including ossification of repair tissue [23]. Second, there is increasing evidence that the use of (fibre) reinforcements in the hydrogels meant for cartilage repair may greatly enhance their efficacy and may even be indispensable [24]. This poses an additional challenge to the fixation efficacy of sealants such as fibrin glue $[25,26]$. Given the importance of the equine model for joint-related disorders and the ethical pressure of reducing the number of animal experiments to the minimum there is, therefore, a need for the critical assessment and, where possible, optimisation of fixation methods for hydrogel-based scaffolds, with and without reinforcement, in an equine cartilage defect model.

The data presented in this article emanate from a series of pilot studies focusing on the optimisation of the fixation of hydrogel-based scaffolds in (osteo)chondral defects. Starting with a widely described and seemingly harmless commercial fibrin glue (CFG) that is considered the standard for fixation, we discovered that use of the material in the specific equine large animal model is fraught with difficulties (study 1). Based on this observation, we proceeded to comparing commercial and autologous fibrin glue (AFG) from an immunogenic point of view in an equine ectopic model (study 2). Finally, we 
compared the efficacy of the AFG with a custom-made alternative fixation method for specific fiber-reinforced hydrogel constructs based on a 3D-printed polycaprolactone (PCL)-based anchor for the fixation of PCL-reinforced hydrogels (study 3).

\section{MATERIALS AND METHODS}

\section{Animal experiments}

Use of CFG for the fixation of hydrogel-based constructs in an orthotopic equine model

The study had been approved by the ethical and animal welfare of the National University of Costa Rica. Nine criollo horses (age 4-10 years, weight 275-375 kg) were used for surgery. The horses were free of lameness and without any clinical or radiographic evidence of acute or chronic injuries. They were housed in individual boxes, and fed a standard maintenance ration of concentrate with hay ad libitum and free access to water. General anaesthesia was induced with diazepam $(0.05 \mathrm{mg} / \mathrm{kg})$, ketamine $(2.2 \mathrm{mg} / \mathrm{kg})$ and lidocaine $(2 \mathrm{mg} / \mathrm{kg})$, after premedication with acepromazine $(0.025 \mathrm{mg} / \mathrm{kg})$ and xylazin $(1.1 \mathrm{mg} / \mathrm{kg})$; anaesthesia was maintained with isoflurane in oxygen with an end tidal concentration of $1.0-1.5 \%$. The medial trochlear ridge of the stifle joint was exposed by arthrotomy through a subpatellar approach; two full thickness cylindrical cartilage defects with a diameter of $6 \mathrm{~mm}$ were created using a manual drill guided by a drill sleeve. Remnants of cartilage in the defect were removed using a sharp surgical spoon. In each horse one of the defects was filled with a 3D printed porous constructs made of one of three different hydrogels (M1OP10-HAMA, StarPEG/heparin, and P(AGE/G)-HA-SH), which had been previously tested both in vitro and in vivo for safety and biocompatibility and were deemed safe), so $n=3$ per gel, and fixated with CFG (Tissucol ${ }^{\mathrm{TM}}$, Baxter), the other (control) defect was filled with fibrin glue $(0.3 \mathrm{~mL})$ only $(n=9)$. The glue was allowed 10 min for cross-linking; the wound was then closed in four layers and full weight bearing was allowed after recovery from anaesthesia. Post-operatively, horses received antibiotics for 3 days (procaine penicillin 15,000 IU/kg IM, SID, and gentamicin $6.6 \mathrm{mg} / \mathrm{kg} \mathrm{IV}, \mathrm{BID}$ ) and nonsteroidal anti-inflammatory drugs (phenylbutazone $2.2 \mathrm{mg} / \mathrm{kg}$, PO BID) during the first 5 days. The animals were subjected to daily monitoring of clinical 
parameters (temperature, heart rate and respiratory rate). After 7, 14 and 28 days ( $n=3$ with one animal/gel per time point), the horses were euthanized and the stifle joints were harvested, fixated in formalin $4 \%$ and processed for histology.

\section{Comparison of commercial and AFG in an equine ectopic model}

This study aimed at comparing the in vivo tissue reaction, safety and degradation of commercial and AFG in an ectopic equine model. This study had been approved by the local Ethics Committee for Animal Experimentation of Utrecht University and was performed in accordance with the Institutional Guidelines on the Use of Laboratory Animals in compliance with the Dutch Act on Animal Experimentation.

\section{- $A F G$ production}

The protocol for production of the fibrin component of the fibrin glue was obtained by adapting the method described by Thorn et al. [27] with the addition of a cryoprecipitation step to enhance fibrin precipitation [28].

A blood sample $(40 \mathrm{~mL})$ was collected from each animal in a tube previously filled with heparin $(20 \mathrm{IU} / \mathrm{mL}$ of blood) and medical grade citrate to obtain a citrate concentration of $3.2 \%$. The plasma was separated by centrifugation for $18 \mathrm{~min}$ at $400 \mathrm{~g}$ at room temperature. The top half of the plasma layer was then transferred by pipetting into a new tube, carefully avoiding the surface containing platelets. Cryoprecipitation was performed by storing overnight the sample at $-20^{\circ} \mathrm{C}$ [28]. The sample was then allowed to thaw at room temperature; next, we initiated precipitation by adding $176 \mu \mathrm{l}$ of $100 \%$ ethanol and, subsequently, mixing by inversion and placing on ice the plasma for 20 min. Centrifugation for $10 \mathrm{~min}$ at $1000 \mathrm{~g}$ followed to allow formation of a fibrin pellet on the bottom of the tube [27]. The pellet was isolated, warmed at $37^{\circ} \mathrm{C}$ to allow solubilisation, and loaded in a two-syringe system together with a commercially available thrombin solution (Tisseel ${ }^{\mathrm{TM}}$, Baxter). The system was kept at $37^{\circ} \mathrm{C}$ with warm water and transferred to the surgical theatre.

\section{- Surgical procedure}

Two adult equines were sedated with detomidine $(10 \mu \mathrm{g} / \mathrm{kg})$. Under local anaesthesia achieved by subcutaneous injection of $1 \mathrm{~mL}$ of mepivacaine solution $(20 \mathrm{mg} / \mathrm{mL})$ in the dorsal region of the neck, a series of subcutaneous 
pockets were created through small incisions on the skin $(6 \mathrm{x}, \sim 10 \mathrm{~mm}$ in length). A small quantity of $0.3 \mathrm{~mL}$ of fibrin glue (commercial or autologous, randomised, $n=3$ ) was deposited in each pocket and allowed to cross-link for 5 min, after which the wounds were sutured. Animals were monitored daily for signs of reaction (temperature, swelling and general aspect of incision area).

After 14 days, the two animals were euthanized by administration of pentobarbital (50 mg/kg of body weight), and the entire soft tissue area containing the constructs was harvested for analysis.

Comparative fixation study with AFG and $3 D$ printed osteal PCL anchor in an equine orthotopic model

This study aimed at comparing the fixation potential of two techniques (osteal anchor and autologous fibrin glue) for the fixation of reinforced hydrogel-based scaffolds intended to be used for articular cartilage repair. These studies had been approved by the Local Ethics Committee for Animal Experimentation of Utrecht University and were performed in accordance with the Institutional Guidelines on the Use of Laboratory Animals in compliance with the Dutch Act on Animal Experimentation.

\section{- Preparation of osteal anchor}

Osteochondral plugs were fabricated by extrusion-based 3D printing of GMPgrade PCL (Purasorb ${ }^{\circledR}$ PC 12, Corbion, The Netherlands) using a screw-based extruder on a 3D Discovery printer (regenHU, Switzerland). The osteochondral plug was designed on BioCAD software (regenHU, Switzerland) as a cylinder with $6 \mathrm{~mm}$ diameter, featuring a square-grid scaffold structure with six zones with different porosity. The lower zones were designed for bone osteoconduction and formed a gradient of decreasing porosity from the bottom to the top, mimicking the transition from trabecular to cortical bone. The top zone of the scaffold represented the endochondral interface, and was designed as completely closed in order to separate the hydrogel materials for cartilage repair from the osteal anchor (Fig. 1A). The uppermost zone in the osteochondral plug was designed for fibre reinforcement of the chondral portion (Fig. 1B), to enhance fixation of the hydrogels and to increase biomechanical resistance of the chondral layer [29].

Before printing, the $\mathrm{PCL}$ was first molten in the extruder heating tank at $90^{\circ} \mathrm{C}$ for at least $30 \mathrm{~min}$ to ensure consistent material viscosity. The osteochondral 
plugs were then fabricated using the following printing parameters: feeding pressure of 0.5 bar, $32 \mathrm{G}$ extrusion nozzle, temperature of $80^{\circ} \mathrm{C}$, spindle speed of $4 \mathrm{rpm}$, and printing speed of $4 \mathrm{~mm} / \mathrm{s}$.
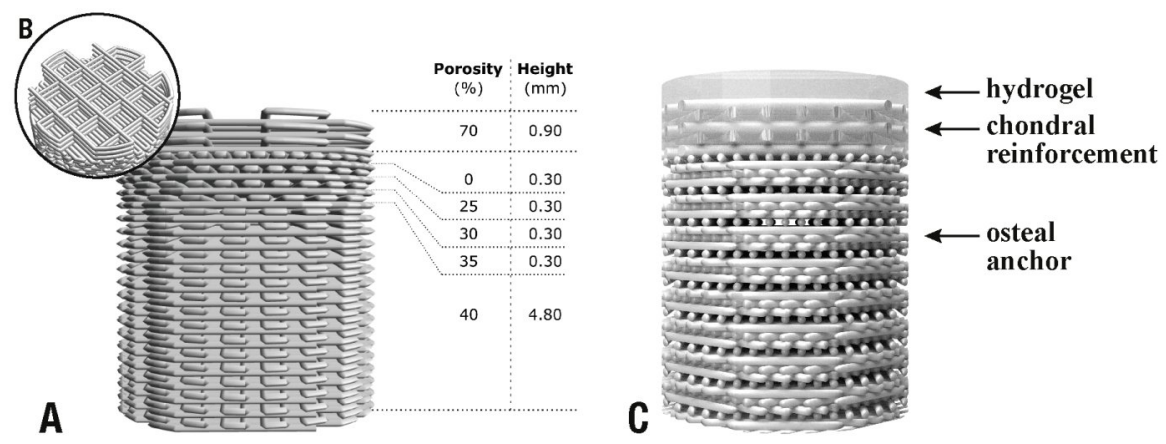

Figure 1. The osteal anchor was designed to have a decreasing porosity leading to a closed interface between the osteal and chondral parts of the construct, to mimic the natural architecture of the subchondral bone (A), as showed in the 3D-model of the PCL-based anchor design (A). A fiber reinforcement was added in the chondral layer to enhance fixation of hydrogel materials and biomechanical resistance of the chondral layer $(B)$. The hydrogel materials can be cast on top (C). AFG, autologous fibrin glue; PCL, polycaprolactone.

- Preparation of chondral and osteochondral hydrogel reinforced constructs for implantation

To compare fixation potential of $\mathrm{AFG}$ and $\mathrm{PCL}$ osteal anchor, reinforced hydrogels constructs were prepared. The hydrogels selected for this purpose were M10P10-HAMA [30], StarPEG/heparin [31], and P(AGE/G)-HA-SH [32].

The osteochondral plugs were inserted into a polytetrafluoroethylene (teflon) custom-made mould of $7.5 \mathrm{~mm}$ height. This system allowed to cast hydrogel materials in a confined area, thus obtaining a uniform layer integrated with the osteal anchor (Fig. 1C). Casting of M10P10-HAMA and $\mathrm{P}(\mathrm{AGE} / \mathrm{G})-\mathrm{HA}-\mathrm{SH}$ was performed infusing $0.42 \mu \mathrm{l}$ of polymer solution mixed with a photoinitiator $\left(0.05 \% \mathrm{w} / \mathrm{w}\right.$, Irgacure ${ }^{\circledR 2} 2959$, BASF, Ludwigshafen, Germany). Subsequently, chemical cross-linking was induced with a UV lamp at $3 \mathrm{~cm}$ distance (UV-Handleuchte Lamp, A. Hartenstein, Germany; wavelength: $365 \mathrm{~nm}$, intensity at $3 \mathrm{~cm}: 1.2 \mathrm{~mW} / \mathrm{cm}^{2}$ ). Casting of starPEG/heparin was achieved by mixing equal quantities of the two polymer solutions, which then cross-linked by click reaction.

To allow direct comparison of the two fixation methods, also when using 
AFG the hydrogels were cast in a mould of $1.5 \mathrm{~mm}$ height together with the PCL reinforcement following the methodology described previously. The constructs were then transported to the surgical theatre for implantation.

\section{- Surgical procedure}

Three Shetland ponies (age 3-10 years, weight 170-240 kg) were used. The horses were free of lameness and without any clinical evidence of acute or chronic injuries. They were housed in individual boxes and fed a standard maintenance ration of concentrate with hay ad libitum and free access to water.

Two defects were made in the medial trochlear ridge of the femur of each stifle joint. On one side, hydrogel based reinforced constructs were implanted and fixated with AFG ( $n=2$ per joint, distally and proximally, one material for each animal); on the contralateral side, the same constructs were implanted and fixated with the osteal anchor.

General anaesthesia was induced with midazolam and ketamine intravenously $(0.06 \mathrm{mg} / \mathrm{kg}+2.2 \mathrm{mg} / \mathrm{kg})$, after premedication with detomidine and morphine $(10 \mathrm{mcg} / \mathrm{kg}+0.1 \mathrm{mg} / \mathrm{kg})$; anaesthesia was maintained with isoflurane in oxygen with an end tidal concentration of 1.0-1.5\%. The medial trochlear ridge of the stifle joint was exposed by arthrotomy through a subpatellar approach; on one side two full thickness cylindrical cartilage defects with a diameter of $6 \mathrm{~mm}$ were created using a drill guided in a drill sleeve. On the contralateral side two osteochondral defects with a diameter of $6 \mathrm{~mm}$ and a depth of $7.5 \mathrm{~mm}$ were created using a surgical drill guided with a $7.5 \mathrm{~mm}$ custom-made drill.

Defects fixated with fibrin glue were filled with $0.2 \mathrm{~mL}$ of AFG, prepared as described previously, after which the hydrogel construct was immersed in the defect and left to sit for 10 min to allow cross-linking and fixation. On the contralateral side, the osteal anchor with the hydrogel constructs was inserted press-fit into the defects.

The wounds were then closed in 4 layers, and full weight bearing was allowed after recovery from anaesthesia. Post-operatively, the ponies received NSAIDS (meloxicam, $0.6 \mathrm{mg} / \mathrm{kg}, \mathrm{PO}, \mathrm{BID}-\mathrm{SID}$ ) up to 7 days and opiates (tramadol, $5 \mathrm{mg} / \mathrm{kg}, \mathrm{PO}, \mathrm{BID}-\mathrm{SID}$ ) up to 3 days postoperatively. For antibiotic prophylaxis, ampicillin $(10-15 \mathrm{mg} / \mathrm{kg})$ was administered intravenously once 
before and procaine penicillin $(20 \mathrm{mg} / \mathrm{kg}, \mathrm{IM})$ once after surgery.

Clinical parameters of the animals were monitored daily for signs of inflammation and lameness. After 14 days, the animals were euthanized by administration of pentobarbital ( $50 \mathrm{mg} / \mathrm{kg}$ of body weight), and the entire osteochondral area containing the constructs was harvested for analysis with the aid of a surgical bone saw.

With the aim of evaluating the bone integration for a longer period, a followup study of 4 weeks was performed on three other ponies (age 4-10 years, weight 190-240 kg), where only the $\mathrm{PCL}$ osteal anchor implantation was performed bilaterally, following the methodology described above.

\section{Post-mortem processing}

\section{Microcomputerized tomography}

Each construct with osteal anchor was scanned before and after implantation in a micro-CT scanner ( $\mu$-CT 80 , Scanco Medical AG, Switzerland) at a resolution of $20 \mu \mathrm{m}$. All defects were scanned post-mortem. The acquisition parameters were set to a voltage of $70 \mathrm{kVp}$, an intensity of $114 \mu \mathrm{A}$ and an integration time of 300 ms. Subsequently, the acquired images were processed by first applying a Gauss filter (sigma $=2$, support $=0.8$ voxel) and then segmentation. A global threshold of 55 per mile and 120/mile was used for the constructs before and after implantation, respectively. The segmented images of the constructs before implantation were also used to determine the porosity of the printed chondral reinforcement and osteal region. The adjacent healthy bone tissue was also scanned. The images obtained were processed with Image to obtain the bone volume data before and after implantation.

\section{Histological processing and stainings}

Soft tissue samples were fixated in $4 \%$ formalin, dehydrated through a graded ethanol series, cleared in xylene and embedded in paraffin. Osteochondral samples for histology were fixated in $4 \%$ formalin, decalcified with Formical 2000 (EDTA/formic acid, Decal Chemical Corporation, Tallman, NY) for 14 days, dehydrated through a graded ethanol series, cleared in xylene and embedded in paraffin. Samples were sectioned into $5 \mu \mathrm{m}$ slices and stained with hematoxylin and eosin (HE) to allow for morphological analysis and the evaluation of tissue-scaffold integration according to a modified 
Drobnic's scoring $[16,33]$ and cell infiltration using an Olympus BX51 light microscope. Samples were also stained with picrosirius red and analyzed with polarized light microscopy for visualization of collagen fibril orientation.

\section{RESULTS}

\section{The use of CFG for the fixation of hydrogel-based constructs in an orthotopic equine model}

Clinical parameters of the animals were normal for the duration of the experiment, with no evidence of lameness or inflammation. Macroscopically, all defects were filled with tissue. Microcomputed tomography (micro-CT) showed signs of bone loss in the subchondral area directly beneath the defect, irrespective of the hydrogel used (Fig. 2). There was some individual variability, but the phenomenon was seen in all horses. In addition, no correlation with specific implanted materials was found. This bone loss (Fig. 3A-D; Supplementary Table S1 and S2) was also consistently visible in all control defects only filled with the fibrin glue (Fig. 3E-H).

At histology, the middle of the defect displayed an area of bone reaction surrounding the site of bone loss (Fig. 3C, G). As shown by the HE staining, this reaction was characterized by infiltration of fibroblasts and predominantly neutrophil granulocytes (Fig. 3D, H).

\section{Comparison of commercial (CFG) and AFG in an equine ectopic model}

No local or systemic adverse reactions were observed in the horses during the experiment. Upon harvest, the excised tissue appeared macroscopically normal. Histological evaluation with HE staining of the CFG showed that the glue was clearly identifiable within the tissue (Fig. 4; Supplementary Fig. S2). It appeared contracted and was surrounded by a front of neutrophil granulocytes (Fig. 4A, arrow). The AFG was not clearly identifiable through different sections of the implantation area, although a structure compatible with its expected appearance was detected in the soft tissue (Fig. 4B, C). The neighbouring tissue did not display any abnormal cell infiltration. 


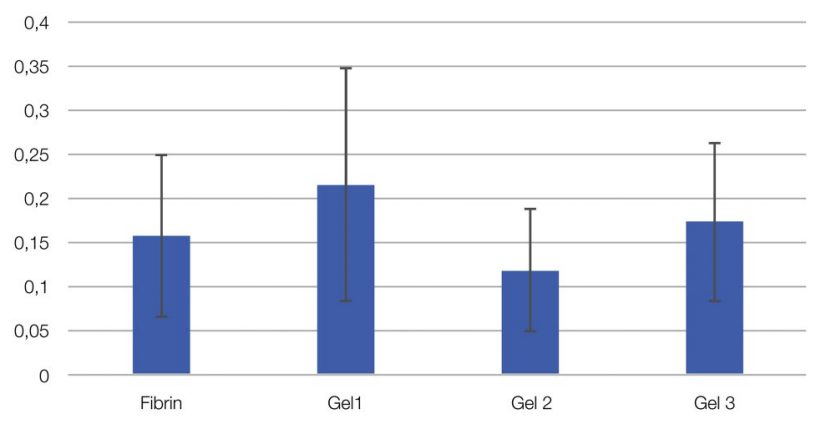

Figure 2. Bone volume loss from study 1. The area beneath the defects and healthy adjacent tissue was scanned postmortem with micro-CT imaging. Images obtained were processed with ImageJ to obtain bone volume values before and after implantation. Statistical analysis showed no correlation of bone loss with any of the materials. micro-CT, microcomputed tomography.

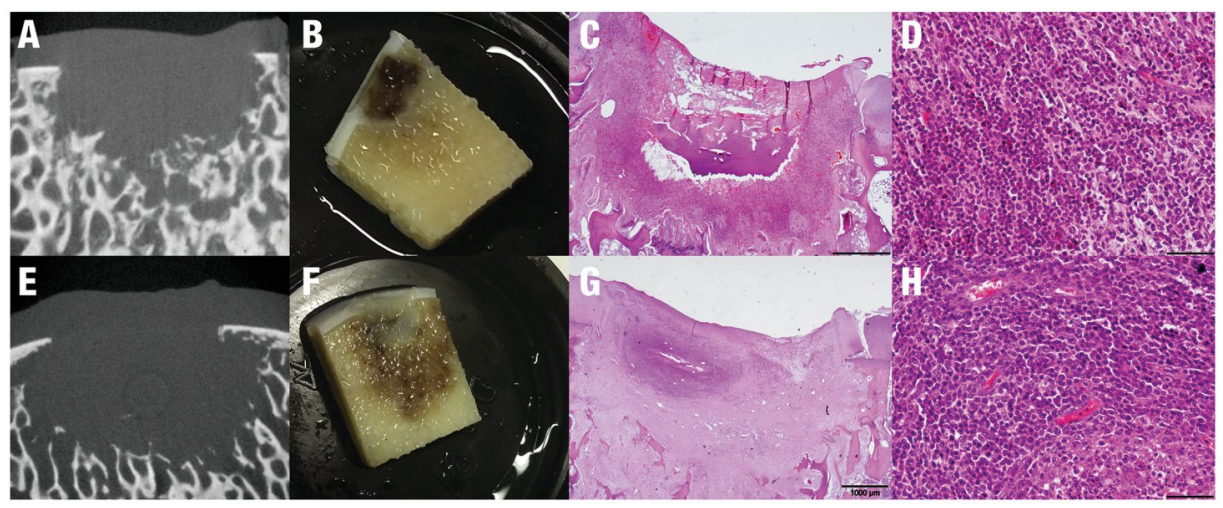

Figure 3. Inflammatory reaction in control defects filled with CFG (14 days postoperatively). First and second rows show representative examples from two different animals. micro-CT imaging showed loss of the trabecular structure and bone resorption (A, E). Upon sectioning the bone loss was confirmed, and a reaction of the surrounding area with inflammation was visible to the naked eye $(B, F)$. HE staining showed a focal reaction $(C, G)$ with recruitment of neutrophil granulocytes with loss of architecture and bone structure (D, H). CFG, commercial fibrin glue; $H E$, hematoxylin and eosin.

\section{Comparative fixation study with AFG and 3D-printed osteal PCL anchor in an equine orthotopic model}

\section{PCL osteal anchor and constructs for implantation}

3D osteochondral constructs with a hierarchical porous architecture were successfully fabricated by means of an extrusion-based 3D printing technique (Fig. 5). Design (Fig. 1) and built architecture (Fig. 5A) revealed the high 


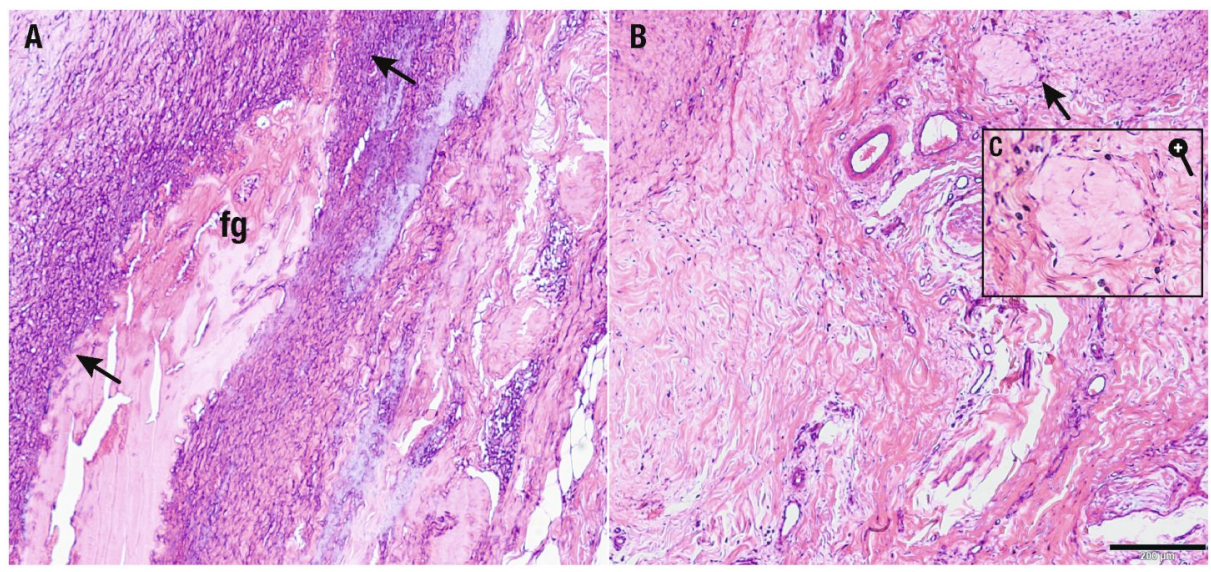

Figure 4. Histological sections of commercial ( $A$, left) and autologous (B, right) fibrin glue, implanted in an equine ectopic model for 14 days. CFG appears contracted and is easily recognizable (A, $f g)$; the glue is surrounded by a front of neutrophil granulocytes ( $A$, black arrows); this is not present in the area where the autologous fibrin was implanted (B), where some macrophages and fibroblasts are present (C) (black bar $=200 \mathrm{~m})$.
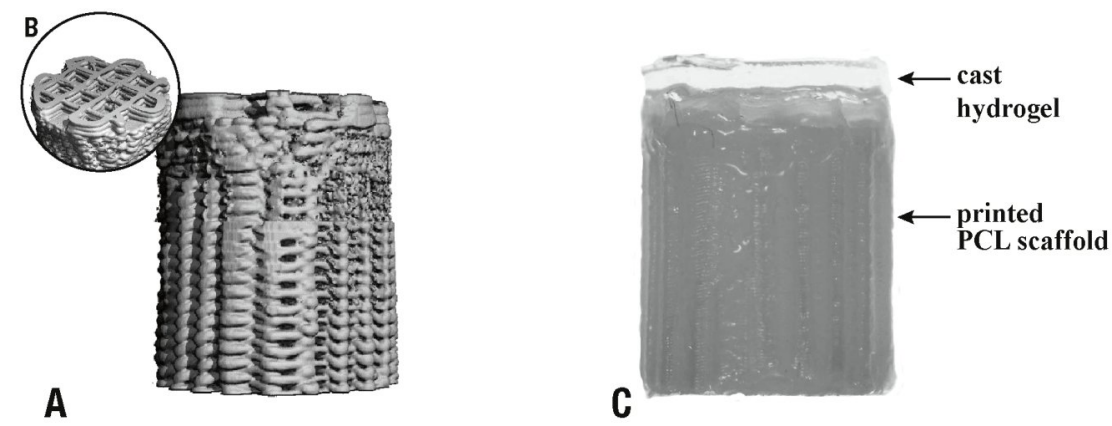

Figure 5: Micro-CT 3D render of the osteochondral anchor before implantation (lateral view, $A$ and top view, B) showing the closure of the layers to allow casting of hydrogels. Aspect of the $P C L$-based osteal anchor with cast hydrogel on top before implantation (C).

accuracy of the printing technique. From the micro-CT data of the printed constructs porosity was also assessed and compared to the design values. Chondral and osteal regions showed porosities of $32 \pm 3 \%$ (designed $40 \%$ ) and $66 \pm 2 \%$ (designed $70 \%$ ), respectively. Furthermore, the 3D reconstructions showed that interconnected pores are presented on the lateral surface of the constructs (Fig. 5A). This is an important factor for the successful mechanical interlocking between the osteal anchor and surrounding natural bone tissue at 
construct/bone interface.

\section{Animal study}

Surgical implantation of the constructs was successful. AFG was kept at $37^{\circ} \mathrm{C}$ before implantation, and mixed with the thrombin solution in the defect, to allow immersion of the hydrogel reinforced constructs (Fig. 6A, B). Osteochondral constructs were fixated by press-fit, exerting pressure with a forceps on the PCL osteal anchor to avoid damage to the hydrogel portions (Fig. 6C, D).

In the 2-week study all animals had an uneventful course of the experiment, temperature and other parameters were well within normal range. However, when challenged by a flexion test, one animal showed some signs of discomfort on the limb in which the scaffolds had been fixated with the osteal anchor.

At postmortem, the 2-week study comparing the two fixation techniques showed that the fixation potential of AFG was inferior compared to the PCL osteal anchor. Although in two out of three animals AFG could keep the reinforced constructs in place, in one animal both defects were empty. Moreover, in the remaining four defects, the constructs displayed evidence of slipping out of the defects (Fig. 7A). All six hydrogels with PCL reinforcement

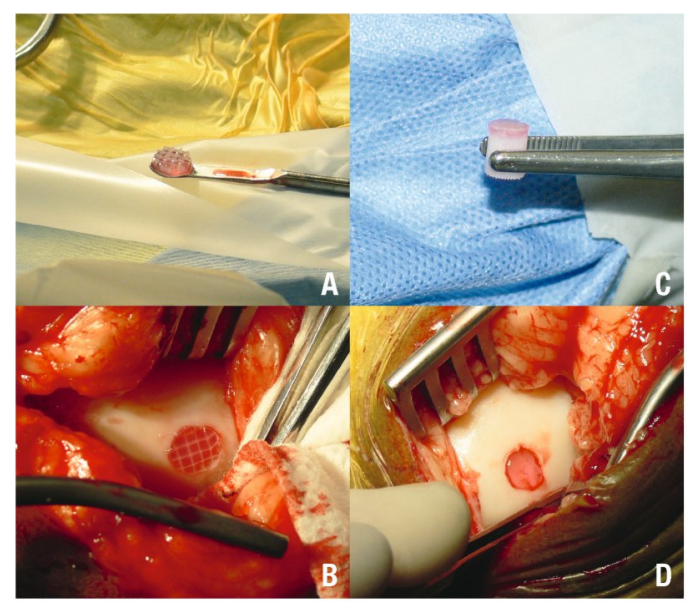

Figure 6. Surgical implantation of materials for comparison of fixation with fibrin glue (left) versus osteal anchor (right). The reinforced hydrogel (A) was implanted in a full-thickness chondral defect and fixated with autologous fibrin glue (B). The hydrogel with PCL osteal anchor (C) was inserted in the osteochondral defect and secured by press fit (D). 

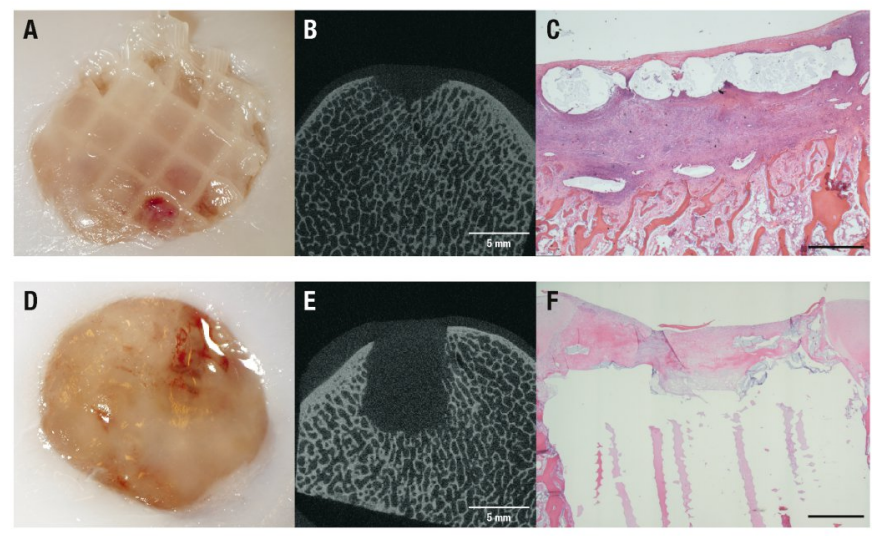

Figure 7. Fixation potential of two techniques, autologous fibrin glue (top) and PCL osteal anchor (bottom), 14 days after implantation. Reinforced chondral constructs appeared still in place in two of three cases; however, the scaffolds looked as if they were starting to slip out proximally (A). micro-CT of AFG fixation showed some bone resorption (B), confirmed by the HE staining (C), which showed loss of architecture directly underneath the defect with significant infiltration of neutrophil granulocytes and fibroblasts. Constructs fixated with the PCL anchor were all still in place $(D)$, and micro-CT imaging showed a conserved trabecular architecture surrounding the construct (E). The chondral portion of the defect appears filled with repair tissue with a predominance of fibroblasts (F). (Black bar=1 $\mathrm{mm}$ ).

\begin{tabular}{|c|c|c|}
\hline Outline attachment & Area coverage & Scaffold integrity \\
\hline unchanged (5) & unchanged (5) & unchanged (5) \\
\hline$<25 \%(4)$ & $<75-100 \%(4)$ & $\begin{array}{l}\text { minor deformities } \\
\text { unrelated to fixation (4) }\end{array}$ \\
\hline $25-50 \%(3)$ & $50-75 \%(3)$ & $\begin{array}{l}\text { minor cracks } \\
\text { close to fixation site (3) }\end{array}$ \\
\hline $50-75 \%(2)$ & $25-50 \%(2)$ & $\begin{array}{l}\text { fissures that } \\
\text { jeopardize the fixation (2) }\end{array}$ \\
\hline $75-100 \%(1)$ & $<25 \%(1)$ & $\begin{array}{l}\text { fissures and scaffold } \\
\text { disorganization in outer area (1) }\end{array}$ \\
\hline \multirow[t]{2}{*}{$100 \%(0)$} & $0 \%(0)$ & $\begin{array}{l}\text { fissures and scaffold } \\
\text { disorganization in general (0) }\end{array}$ \\
\hline & AFG & PCL \\
\hline outline attachment & $1.7( \pm 1.50)$ & $4.8( \pm 0.41)$ \\
\hline area coverage & $2.7( \pm 2.06)$ & $4.8( \pm 0.41)$ \\
\hline scaffold integrity & $2( \pm 1.79)$ & $4( \pm 0)$ \\
\hline total score & $6.3( \pm 5.12)$ & $13.7( \pm 0.82)^{*}$ \\
\hline
\end{tabular}

Table 1. Criteria for fixation scoring according to a Modified Drobnic Scoring. Scores show the difference between AFG and PCL efficacy in fixation. AFG shows high variability in results; overall efficacy for fixation with PCL was significantly higher than with AFG $\left({ }^{*} p<0.05\right)$. 
fixated by means of the PCL anchor were still in place after 2 weeks (Fig. 7D). In the single animal that showed discomfort at the flexion test, the soft tissue opposing the defect showed signs of inflammation.

Micro-CT of the defects and surrounding area in the AFG group (Fig. 7B) showed again the subchondral inflammatory reaction that had been observed in the first study in which CFG had been used, although to a lesser extent. The defects in the PCL group showed no signs of bone reaction (Fig. 7E).

Semiquantitative fixation scores showed that PCL gave better results both from an attachment point of view and from a scaffold integrity aspect (Table 1). One-way ANOVA confirmed a significant difference between the two groups $(p<0.05)$.

Histological analysis confirmed the findings from the micro-CT. In the AFG group, an inflammatory response was observed in the bone underlying the defect, characterized by the presence of fibroblastic cells, neutrophils and multinucleated cells (Fig. 7C). Bone resorption was consistently present in all defects. In the PCL group, a fibroblast-based infiltration from the bone surrounding the osteal part of the plug was observed, to a degree that can be expected after implanting an osteal scaffold (Fig. 7F; Supplementary Fig. S1).

In the follow-up 4 week study none of the animals showed any clinical sign. Micro-CT imaging showed signs of mineralization within the PCL osteal anchor (Fig. 8A), which was confirmed upon histology (Fig. 8B, C).

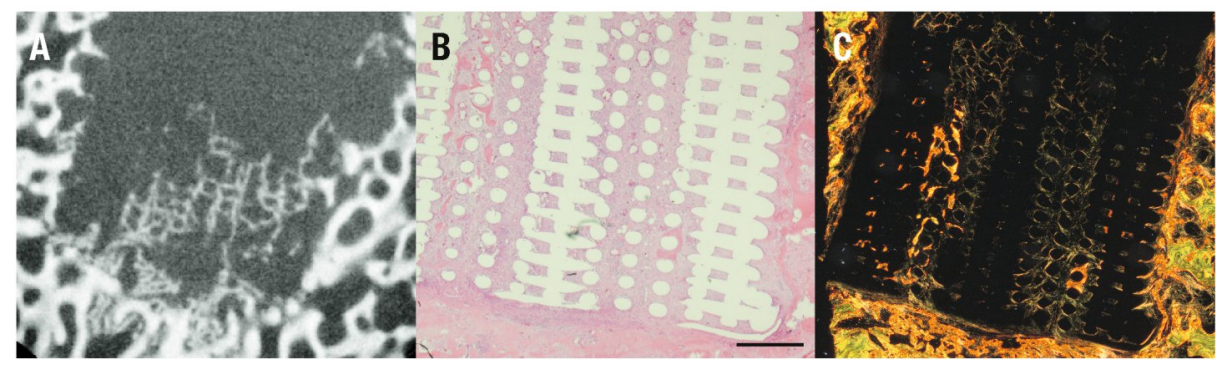

Figure 8. Bone regeneration after 28 days. micro-CT imaging showed signs of mineralization and new bone formation within the PCL osteal anchor (A). HE staining showed good integration of the anchor with the surrounding tissue (B) and picrosirius red staining under polarized light confirmed presence of new bone $(C)$ (Black bar=1 $\mathrm{mm}$ ). 


\section{DISCUSSION}

In the quest for the optimal tissue-engineered construct that can be implanted in an articular cartilage defect to incite regeneration of the tissue, the fixation of such constructs within the surrounding non-degraded native tissue is a pivotal issue. Improper fixation will inevitably lead to implant failure, irrespective of the qualities of the materials and/or cells that are to be tested [34]. In large animal models, fixation is especially important as loading is almost immediate after surgery and as constructs are exposed to substantial biomechanical compressive and shear forces. Thus far, the focus in the field has predominantly been directed at material development, production methods (e.g., 3D bio-printing), structural aspects of the construct (e.g., zonal composition) and cellular aspects (e.g., use of differentiated versus undifferentiated cells) $[35,36]$, but surprisingly little attention has been given to the fixation of the implants.

In the advanced techniques used for cartilage defect repair in human healthcare, such as autologous chondrocyte implantation (ACl) and matrixassisted $\mathrm{ACl}(\mathrm{MACl})$ the use of fibrin glue is regarded as the gold standard for fixation of implanted cells and/or materials [20, 37, 38]. For this purpose, commercially available fibrin glue obtained from human blood components is used. In many animal studies in which various types of implants for cartilage repair were assessed this practice has been copied without further investigation, notwithstanding the fact that by doing so the use of fibrin glue changed from allogeneic in humans to xenogeneic in any other animal [19, 39, 40]. Long-term results in some studies did not give rise to suspicions that the use of commercial fibrin glue (CFG) might have negative side effects [18], but no short-term assessments of a possible acute reaction to the product have been performed and there were some indications that the use of the product might be less innocuous than assumed in both goats and horses [21], (Mcllwraith, pers. comm. 2016). In this article we present data from a series of short term studies that were originally executed as preliminary work for long term experiments focusing on the assessment of hydrogels for cartilage repair. Well aware of the limitations of these studies that were originally never meant to be stand-alone studies, we nevertheless felt it useful to report the outcome to the scientific community, given the unexpected events at short term after implantation that came to light, the importance of construct fixation in 
cartilage repair, and the need to avoid as much as possible unnecessary wastage of experimental animals.

In the first orthotopic study the panel of cells that had infiltrated the defects in which CFG had been used for fixation, showed a large predominance of neutrophils that suggest a recruitment of inflammatory cells far beyond expectations 1-4 weeks post-implantation. The most striking point was that this inflammatory reaction, which was accompanied by severe bone loss, was similar in all defects, irrespective of the hydrogel used. Two possible explanations for this phenomenon were formulated based on the observations and literature. The first potential explanation was that the use of xenogeneic material (human origin glue in an equine model) might have led to an inflammatory reaction based on an immune response, as had been supposed by Brehm et al. [21] in their goat model. Alternatively, the subchondral bone reaction might have been caused by the mechanical stress exerted by weight bearing on the exposed bone triggering a biological response [41]. Vasara et al. [42] described how a subchondral reaction with bone resorption was detected in goats, 3 months after implantation. Moreover, Kold et al. [43] reported the formation of cystic lesions in the femoral condyle of horses after creating a slitlike lesion in the articular cartilage. Timewise, it might have been the case that in our study the bone did not have sufficient time to complete remodeling and hence the bone resorption was more evident than in other reported studies. Since bone remodeling occurs starting from the third or fourth week [44], it may even be possible that this phenomenon is more common than suspected, but may go undetected, as most large animal studies are longer than 4 weeks [45]. However, in our pilot study lesions were created on the medial trochlear ridge of the femur, which is an area that is not or hardly loaded during rest and only affected by principally shear forces during locomotion. For this reason, we focused first on the CFG as potential cause of the heavy reaction.

To realize a study on the potential inflammatory effect of CFG, a procedure for the production of AFG had to be developed. The use of partially autologous fibrin glue was deemed acceptable, as the most immunogenic component was suspected to be the one containing fibrinogen [46]. We, therefore, elected to still use the commercial thrombin component. While being successful, the efforts to develop a reproducible protocol yielding a usable product confirmed that the synthesis of AFG is laborious, and subject to individual variation in concentration and characteristics, based on the animal's health, nutrition, and 
immune condition at the moment of blood collection [47]. This results in a final product that is not standardized, and that may introduce additional variation to the experiments. This is a disadvantage compared to the use of a commercially available fibrin glue that offers a ready-to-use, consistent product. The difference in cell recruitment observed in the ectopic model between the CFG and AFG, however, strongly suggested that indeed the xenogeneic nature of the CFG resulted in an undesired inflammatory response. This response was clearly less prominent when using AFG, although not entirely nonexistent. This outcome suggests that the fibrin component may indeed have had a predominant role in the provocation of this response, however it also hinted at the presence of some residual immunogenic effect, possibly caused by the still xenogeneic thrombin.

Given the fact that also the use of this form of AFG was not entirely reactionfree and the inherent difficulty of the use of any type of fibrin glue for the fixation of fiber-reinforced scaffolds, a different approach was considered. The first part of the third study compared the efficacy of AFG versus an entirely different, osteochondral concept for the fixation of fiber reinforced hydrogels for cartilage repair. Multi-composite scaffolds for cartilage regeneration envisage solving the problem of the relatively low stiffness that is inherent to hydrogels by altering the architecture of the scaffolds. One of the approaches to overcome this, is the use of fiber reinforcement [29]. Whereas this technology allows for the creation of scaffolds of which the stiffness approaches that of native cartilage [24], it poses an ulterior problem for the fixation of the scaffold. This is particularly important when treating chondral defects that are usually not more than 1-2 mm deep and in which the risk of the reinforcing mesh being swept out by shear forces is real. For this reason, in this study the AFG-based fixation was compared to fixation of the mesh and hydrogel to an osteal anchor that itself was placed press-fit in an osteochondral defect. The PCL osteal anchor was designed to mimic the natural architecture and transition of subchondral bone to cartilage. The reinforcement in the chondral portion was added to enhance integration and mechanical properties of the hydrogel cast on top. In this case, casting was used, but the design can easily be adapted to allow 3D co-printing of the hydrogel and reinforcement fibers [29]. The hydrogels used were the same as in the first study and all of them had been previously tested for safety and biocompatibility in the horse (data not shown), suggesting that the materials 
were not likely to cause reaction. The last fixation study showed that bone resorption was still an issue in the AFG, although the bone destruction seemed considerably less than in the case of CFG. It was concluded that apparently only replacing the fibrin component of the glue by an autologous product was not enough to avoid this unwanted side effect, and/or that the influence of the mechanical component was larger than anticipated. Another important finding was that the fixation potential of the AFG was apparently not enough to keep the fiber reinforcement in place in all cases. After 14 days $33 \%$ of the defects were empty, and the remaining constructs were in place but showed early signs of the mesh slipping out. In that aspect, the PCL fixation group showed more promising results, with a good integration of the anchor with the surrounding tissue at early stages, which were confirmed by the second, 4week follow-up, study. The infiltration of the PCL osteal anchor by tissue and the formation of new bone suggest a good integration and adequate response of the native tissue to the construct. However, a long-term evaluation is necessary to confirm these preliminary findings and draw more definitive conclusions [48].

The PCL anchor technique solves the fixation issue, as the construct is kept in place by the press-fit fixation of the anchor in the osteochondral defect, but it should be realized that this is a conceptually different approach that is valid for osteochondral defects only and not for chondral ones. There has been debate in literature whether chondral or osteochondral defects lead to better results in cartilage repair $[43,49]$, but there is no consensus. An equine study in which the spontaneous repair after 1 year of standardized chondral and osteochondral lesions in the same animal was compared showed better cartilage healing in the osteochondral defects, but these also featured almost invariably subchondral bone changes, which were seen to a much lesser extent in the chondral lesions [50].

Taken together, it can be concluded from the pilot investigations reported in this study that the use of xenogeneic fibrin glue for the fixation of scaffolds intended for regeneration of articular cartilage can be advised against. The use of AFG reduces the unwanted side-effects seen with the xenogeneic glue, but its fabrication process is tedious, susceptible to variability, and should probably focus on the manufacturing of an entirely autologous product, as use of a partly autologous product did not result in the complete avoidance of the inflammatory response. Even if this would be solved, the limited capacity of 
any type of fibrin glue to fixate composite scaffolds featuring fiber reinforcement remains an issue. The use of osteochondral plugs that are implanted press-fit solves the fixation issue, but this versatile and surgically relatively easy approach implies the application of an entirely different concept of cartilage healing that includes the total osteochondral unit and not only the chondral part of the joint surface. It is well possible that in the near future, with the advent of sophisticated bioreactor systems that will be able to mimic physiological loading of joint tissues for prolonged periods, the need for large animal in vivo studies for the assessment of potential regenerative therapies for articular cartilage defects will decline. However, at this stage of orthopedic research, no valid alternatives that can fully replace large animal models have been described yet $[51,52]$ and the equine model is still considered as one of the most representative models available [14, 53]. In these large animal models, fixation represents a fundamental issue, and will continue to do so particularly in the perspective of future translation of composite scaffolds to clinical use, where obviously humans will be in first place, followed however by companion animals such as horses and dogs, which are in this respect not only experimental animals, but also patients in their own right.

\section{ACKNOWLEDGEMENTS}

We'd like to thank C. Wayne Mcllwraith BVSc PhD Dipl ACVS/ECVS/ACVSMR for his much appreciated help and constructive comments, and A. Bouwman for the help in data collection of study 1 . The research leading to these results has received funding from the Ministerio de Ciencia, Tecnología y Telecomunicaciones de Costa Rica (MICITT), the Consejo Nacional para Investigaciones Científicas y Tecnológicas de Costa Rica (CONICIT), the European Community's Seventh Framework Programme (FP7/2007-2013) under grant agreement 309962 (HydroZONES), the European Research Council under grant agreement 647426 (3D-JOINT), and the Dutch Arthritis Foundation (LLP-12 and LLP-22). 


\section{REFERENCES}

[1] A.J. Sophia Fox, A. Bedi, S.A. Rodeo, The basic science of articular cartilage: structure, composition, and function, Sports health 1(6) (2009) 461-468.

[2] Z. Yang, Y. Shi, X. Wei, J. He, S. Yang, G. Dickson, J. Tang, J. Xiang, C. Song, G. Li, Fabrication and repair of cartilage defects with a novel acellular cartilage matrix scaffold, Tissue Engineering Part C: Methods 16(5) (2009) 865-876.

[3] A.F. Steinert, S.C. Ghivizzani, A. Rethwilm, R.S. Tuan, C.H. Evans, U. Nöth, Major biological obstacles for persistent cell-based regeneration of articular cartilage, Arthritis research \& therapy 9(3) (2007) 213.

[4] J.S. Temenoff, A.G. Mikos, Review: tissue engineering for regeneration of articular cartilage, Biomaterials 21(5) (2000) 431-440.

[5] G. Smith, G. Knutsen, J. Richardson, A clinical review of cartilage repair techniques, Bone \& Joint Journal 87(4) (2005) 445-449.

[6] E.A. Makris, A.H. Gomoll, K.N. Malizos, J.C. Hu, K.A. Athanasiou, Repair and tissue engineering techniques for articular cartilage, Nature Reviews Rheumatology 11(1) (2015) 21.

[7] E.B. Hunziker, K. Lippuner, M. Keel, N. Shintani, An educational review of cartilage repair: precepts \& practice-myths \& misconceptions-progress \& prospects, Osteoarthritis and cartilage 23(3) (2015) 334-350.

[8] C.K. Kuo, W.-J. Li, R.L. Mauck, R.S. Tuan, Cartilage tissue engineering: its potential and uses, Curr. Opin. Rheumatol. 18(1) (2006) 64-73.

[9] B.V. Slaughter, S.S. Khurshid, O.Z. Fisher, A. Khademhosseini, N.A. Peppas, Hydrogels in regenerative medicine, Advanced materials 21(32-33) (2009) 3307-3329.

[10] L. Kock, C.C. van Donkelaar, K. Ito, Tissue engineering of functional articular cartilage: the current status, Cell and tissue research 347(3) (2012) 613-627.

[11] R. LeBaron, K.A. Athanasiou, Ex vivo synthesis of articular cartilage, Biomaterials 21(24) (2000) 2575-2587.

[12] A. Schwab, Kock, L., Mouser, V.H.M, Stichler, S., Abbadessa, A., Schrön, F., Hansmann, F., Ehlicke, F., and Walles, F., Screening novel hydrogels in an ex vivo cartilage defect model, European Cells and Materials 31(1) (2016) 7.

[13] C.W. Mcllwraith, L.A. Fortier, D.D. Frisbie, A.J. Nixon, Equine models of articular cartilage repair, Cartilage 2(4) (2011) 317-326.

[14] J. Malda, K. Benders, T. Klein, J. De Grauw, M. Kik, D. Hutmacher, D. Saris, P. Van Weeren, W. Dhert, Comparative study of depth-dependent characteristics of equine and human osteochondral tissue from the medial and lateral femoral condyles, Osteoarthritis Cartilage 20(10) (2012) 1147-1151.

[15] E.B. Hunziker, A. Stähli, Surgical suturing of articular cartilage induces osteoarthritis-like changes, Osteoarthritis and cartilage 16(9) (2008) 1067-1073.

[16] J. Bekkers, A. Tsuchida, J. Malda, L. Creemers, R. Castelein, D. Saris, W. Dhert, Quality of scaffold fixation in a human cadaver knee model, Osteoarthritis and cartilage 18(2) (2010) 266272.

[17] J.T. Kerker, A.J. Leo, N.A. Sgaglione, Cartilage repair: synthetics and scaffolds: basic science, surgical techniques, and clinical outcomes, Sports medicine and arthroscopy review 16(4) (2008) 208-216.

[18] A. Nixon, E. Rickey, T. Butler, M. Scimeca, N. Moran, G. Matthews, A chondrocyte infiltrated collagen type $\mathrm{I} / \mathrm{III}$ membrane ( $\mathrm{MACl}^{\circledR}$ implant) improves cartilage healing in the equine patellofemoral joint model, Osteoarthritis and cartilage 23(4) (2015) 648-660.

[19] M. Lind, A. Larsen, C. Clausen, K. Osther, H. Everland, Cartilage repair with chondrocytes in fibrin hydrogel and MPEG polylactide scaffold: an in vivo study in goats, Knee Surgery, Sports Traumatology, Arthroscopy 16(7) (2008) 690-698.

[20] J.E. Bekkers, A.I. Tsuchida, M.H. van Rijen, L.A. Vonk, W.J. Dhert, L.B. Creemers, D.B. Saris, Single-Stage Cell-Based Cartilage Regeneration Using a Combination of Chondrons and Mesenchymal Stromal Cells Comparison With Microfracture, The American journal of sports 
medicine 41(9) (2013) 2158-2166.

[21] W. Brehm, B. Aklin, T. Yamashita, F. Rieser, T. Trüb, R. Jakob, P. Mainil-Varlet, Repair of superficial osteochondral defects with an autologous scaffold-free cartilage construct in a caprine model: implantation method and short-term results, Osteoarthritis and cartilage 14(12) (2006) 1214-1226.

[22] B.W. Hale, L.R. Goodrich, D.D. Frisbie, C.W. Mcllwraith, J.D. Kisiday, Effect of scaffold dilution on migration of mesenchymal stem cells from fibrin hydrogels, American journal of veterinary research 73(2) (2012) 313-318.

[23] L.R. Goodrich, A.C. Chen, N.M. Werpy, A.A. Williams, J.D. Kisiday, A.W. Su, E. Cory, P.S. Morley, C.W. Mcllwraith, R.L. Sah, Addition of Mesenchymal Stem Cells to Autologous PlateletEnhanced Fibrin Scaffolds in Chondral Defects, J Bone Joint Surg Am 98(1) (2016) 23-34.

[24] J. Visser, F.P. Melchels, J.E. Jeon, E.M. Van Bussel, L.S. Kimpton, H.M. Byrne, W.J. Dhert, P.D. Dalton, D.W. Hutmacher, J. Malda, Reinforcement of hydrogels using three-dimensionally printed microfibres, Nature communications 6 (2015).

[25] S.R. Frenkel, P.E. Di Cesare, Scaffolds for articular cartilage repair, Annals of biomedical engineering 32(1) (2004) 26-34.

[26] S. Knecht, C. Erggelet, M. Endres, M. Sittinger, C. Kaps, E. Stüssi, Mechanical testing of fixation techniques for scaffold-based tissue-engineered grafts, Journal of Biomedical Materials Research Part B: Applied Biomaterials 83(1) (2007) 50-57.

[27] J. Thorn, H. Sørensen, U. Weis-Fogh, M. Andersen, Autologous fibrin glue with growth factors in reconstructive maxillofacial surgery, International journal of oral and maxillofacial surgery 33(1) (2004) 95-100.

[28] J.B. Cavichiolo, M. Buschle, B. Carvalho, Comparison of fibrin adhesives prepared by 3 different methods, International archives of otorhinolaryngology 17(1) (2013) 62-65.

[29] W. Schuurman, V. Khristov, M.W. Pot, P.R. van Weeren, W.J. Dhert, J. Malda, Bioprinting of hybrid tissue constructs with tailorable mechanical properties, Biofabrication 3(2) (2011) 021001.

[30] V.H.M. Mouser, A. Abbadessa, R. Levato, W.E. Hennink, T. Vermonden, D. Gawlitta, J. Malda, Development of a thermosensitive HAMA-containing bio-ink for the fabrication of composite cartilage repair constructs, Biofabrication 9(1) (2017) 015026.

[31] E. Hesse, U. Freudenberg, T. Niemietz, C. Greth, M. Weisser, S. Hagmann, M. Binner, C. Werner, W. Richter, Peptide-functionalized starPEG/heparin Hydrogels Direct Mitogenicity, Cell Morphology and Cartilage Matrix Distribution in vitro and in vivo, Journal of Tissue Engineering and Regenerative Medicine (2017).

[32] S. Stichler, S. Bertlein, T. Jüngst, T. Böck, E. Hesse, Y. Renz, E. Seebach, I. Mancini, R. Van Weeren, W. Richter, Thiol-ene cross-linked polyglycidol-hyaluronic acid hybrid hydrogels: Preparation, cell loading, 3D printing, and in vivo evaluation.

[33] M. Drobnič, D. Radosavljevič, D. Ravnik, V. Pavlovčič, M. Hribernik, Comparison of four techniques for the fixation of a collagen scaffold in the human cadaveric knee, Osteoarthritis and cartilage 14(4) (2006) 337-344.

[34] D.W. Hutmacher, Scaffolds in tissue engineering bone and cartilage, Biomaterials 21(24) (2000) 2529-2543.

[35] W.M. Groen, Diloksumpan, P., van Weeren, P.R., Levato, R., and Malda., J, From intricate to integrated: biofabrication of articulating joints, J. Orthop. Res. provisionally accepted (2017).

[36] D.J. Huey, J.C. Hu, K.A. Athanasiou, Unlike bone, cartilage regeneration remains elusive, Science 338(6109) (2012) 917-921.

[37] M. Cucchiarini, H. Madry, F. Guilak, D. Saris, M. Stoddart, M. Koon Wong, P. Roughley, A vision on the future of articular cartilage repair, Eur Cell Mater 27 (2014) 12-16.

[38] E. Kon, G. Filardo, A. Di Martino, M. Marcacci, ACl and MACl, The journal of knee surgery 25(01) (2012) 017-022.

[39] A. Marmotti, M. Bruzzone, D.E. Bonasia, F. Castoldi, M.M. Von Degerfeld, C. Bignardi, S. Mattia, A. Maiello, R. Rossi, G.M. Peretti, Autologous cartilage fragments in a composite scaffold for one stage osteochondral repair in a goat model, Eur Cell Mater 26 (2013) 15-31.

[40] A.J. Nixon, L.A. Fortier, J. Williams, H. Mohammed, Enhanced repair of extensive articular 
defects by insulin-like growth factor-I-laden fibrin composites, Journal of Orthopaedic Research 17(4) (1999) 475-487.

[41] M.B. Fisher, N.S. Belkin, A.H. Milby, E.A. Henning, M. Bostrom, M. Kim, C. Pfeifer, G. Meloni, G.R. Dodge, J.A. Burdick, Cartilage repair and subchondral bone remodeling in response to focal lesions in a mini-pig model: implications for tissue engineering, Tissue Engineering Part A 21(3-4) (2014) 850-860.

[42] A.I. Vasara, M.M. Hyttinen, M.J. Lammi, P.E. Lammi, T.K. Långsjö, A. Lindahl, L. Peterson, M. Kellomäki, Y.T. Konttinen, H.J. Helminen, Subchondral bone reaction associated with chondral defect and attempted cartilage repair in goats, Calcified tissue international 74(1) (2004) 107114.

[43] S. Kold, J. Hickman, F. Melsen, An experimental study of the healing process of equine chondral and osteochondral defects, Equine veterinary journal 18(1) (1986) 18-24.

[44] A.A. White, M. Panjabi, W. Southwick, The four biomechanical stages of fracture repair, J Bone Joint Surg Am 59(2) (1977) 188-192.

[45] B. Ahern, J. Parvizi, R. Boston, T. Schaer, Preclinical animal models in single site cartilage defect testing: a systematic review, Osteoarthritis and cartilage 17(6) (2009) 705-713.

[46] W.H. Seegers, Blood clotting enzymology, Academic Press2013.

[47] T. Meade, R. Chakrabarti, A. Haines, W. North, Y. Stirling, Characteristics affecting fibrinolytic activity and plasma fibrinogen concentrations, Br Med J 1(6157) (1979) 153-156.

[48] R.V. Bolaños, S. Cokelaere, J.E. McDermott, K. Benders, U. Gbureck, S. Plomp, H. Weinans, J. Groll, P. van Weeren, J. Malda, The use of a cartilage decellularized matrix scaffold for the repair of osteochondral defects: the importance of long-term studies in a large animal model, Osteoarthritis and Cartilage 25(3) (2017) 413-420.

[49] D.D. Frisbie, J.T. Oxford, L. Southwood, G.W. Trotter, W.G. Rodkey, J.R. Steadman, J.L. Goodnight, C.W. Mcllwraith, Early events in cartilage repair after subchondral bone microfracture, Clinical orthopaedics and related research 407 (2003) 215-227.

[50] E. Salonius, Rieppo, L., Nissi, M.J., Pulkkinen, H.J., Brommer, H., Brünott, A., Silvast, T.S., van Weeren, P.R., Muhonen, V., Brama, P.A.J., and Kiviranta, I., Insufficient spontaneous repair of even small cartilage lesions in the horse, Am J Sports Med submitted (2017).

[51] J. Cook, C. Hung, K. Kuroki, A. Stoker, C. Cook, F. Pfeiffer, S. Sherman, J. Stannard, Animal models of cartilage repair, Bone and Joint Research 3(4) (2014) 89-94.

[52] C.R. Chu, M. Szczodry, S. Bruno, Animal models for cartilage regeneration and repair, Tissue Engineering Part B: Reviews 16(1) (2010) 105-115.

[53] A. McCoy, Animal Models of Osteoarthritis Comparisons and Key Considerations, Veterinary pathology (2015) 0300985815588611. 


\section{SUPPLEMENTAL DATA}
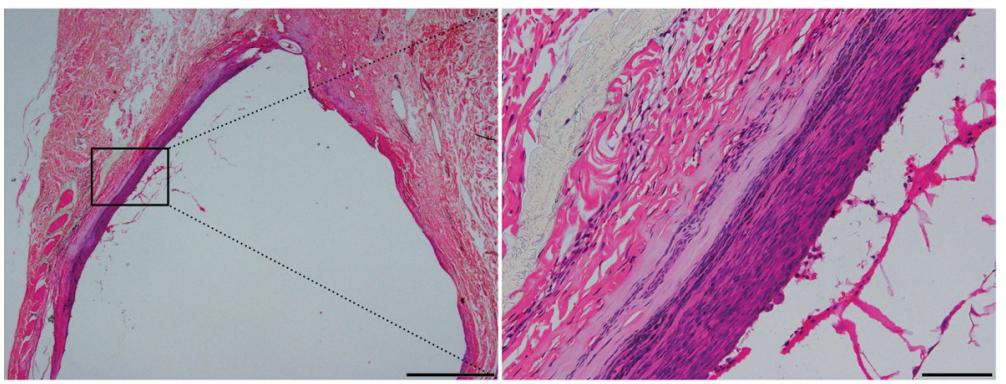

Figure S1. Histological section of tissue surrounding PCL after implantation in an equine ectopic model for 14 days. PCL is dissolved during processing of sections and the remaining front of contact appears infiltrated mostly by fibroblasts, some macrophages, and neutrophils (right black bar $=200$, left black bar $=100$ ).
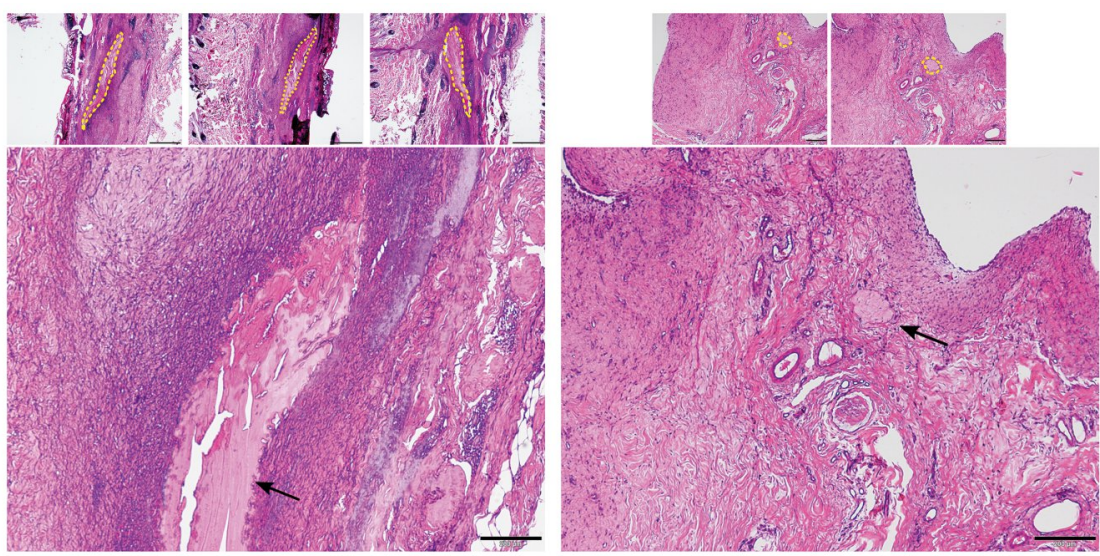

Figure S2. Figure 4 alternative with outlines and illustration of three-dimensional aspect of constructs throughout the tissue.

\begin{tabular}{|c|c|c|c|c|}
\hline Study & Number animals & Location & Duration & Findings and conclusions \\
\hline $\begin{array}{l}\text { Fixation potential of CFG for hydrogel } \\
\text { based constructs }\end{array}$ & 9 & Orthotopic & 1-2-4 weeks & $\begin{array}{l}\text { Some bone loss was detected in all } \\
\text { defects, independently of time, } \\
\text { material or animal. Only common } \\
\text { factor was CFG, used in all defects. }\end{array}$ \\
\hline Comparison of CFG and AFG & 2 & Ectopic & 2 weeks & $\begin{array}{r}\text { Cell recruitment in CFG showed } \\
\text { prevalence of neutrophils and } \\
\text { macrophages. AFG degraded more } \\
\text { rapidly and was difficult to detect, less } \\
\text { immune cells recruitment. }\end{array}$ \\
\hline $\begin{array}{l}\text { Comparison of fixation potential of AFG } \\
\text { and } P C L \text { osteal anchor }\end{array}$ & 3 & Orthotopic & 2 weeks & $\begin{array}{r}\text { Fixation of constructs with AFG proved } \\
\text { to be less efficient than with osteal } \\
\text { anchor. }\end{array}$ \\
\hline
\end{tabular}

Table S1. Overview of studies described in this article. 


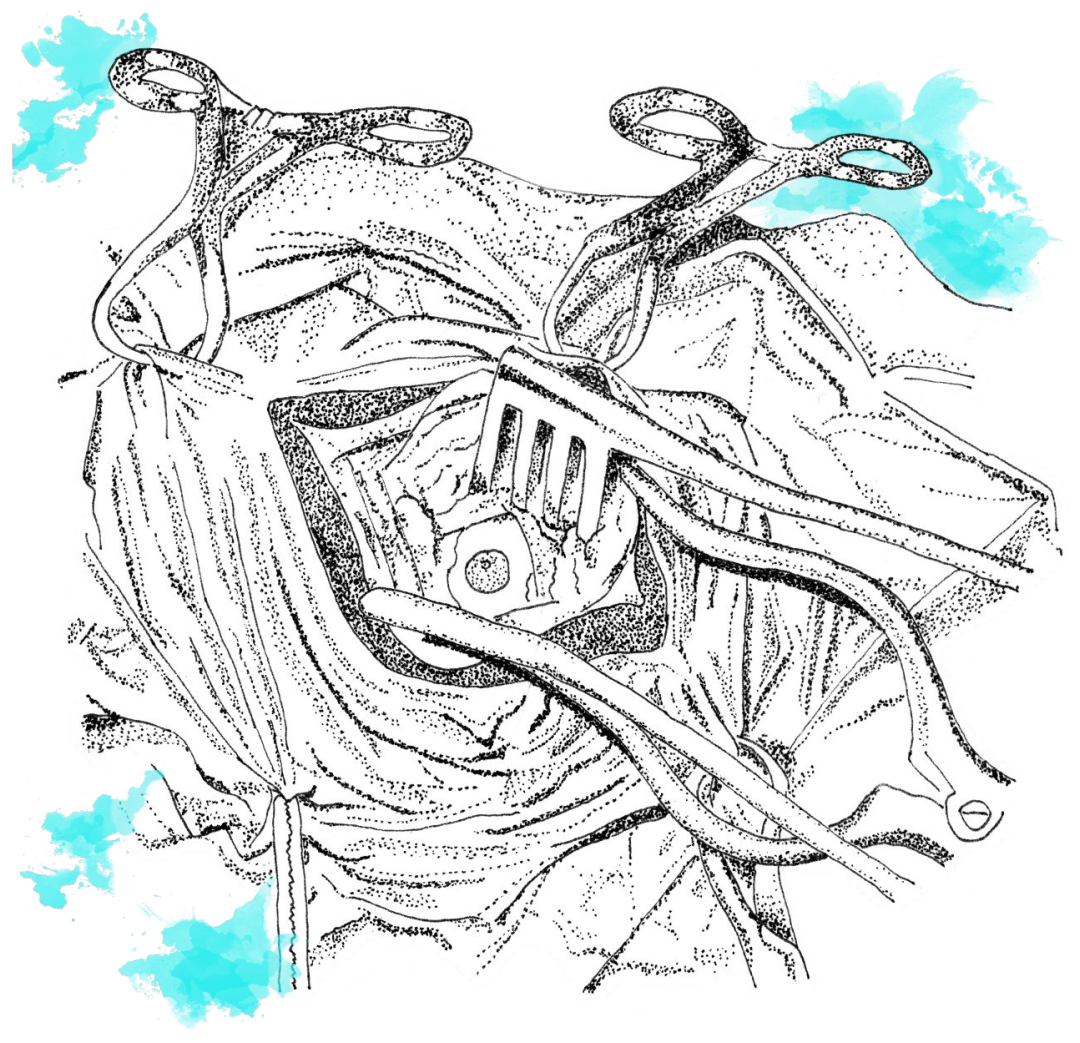




\title{
Chapter
}

\section{A composite hydrogel-3D printed thermoplast osteochondral anchor as example for a zonal approach to cartilage repair: in vivo performance in a long-term equine model}

\author{
I.A.D. Mancini \\ S. Schmidt \\ H. Brommer \\ B. Pouran \\ S. Schäfer \\ J. Tessmar \\ A. Mensinga \\ M.H.P. van Rijen \\ J. Grol \\ T. Blunk \\ R. Levato \\ J. Malda \\ P.R. van Weeren
}

Biofabrication, 2020, 12(3):035028 


\section{ABSTRACT}

Recent research has been focusing on the generation of living personalized osteochondral constructs for joint repair. Native articular cartilage has a zonal structure, which is not reflected in current constructs and which may be a cause of the frequent failure of these repair attempts.

Therefore, we investigated the performance of a composite implant that further reflects the zonal distribution of cellular component both in vitro and in vivo in a long-term equine model.

Constructs constituted of a 3D-printed poly( $\varepsilon$-caprolactone) (PCL) bone anchor from which reinforcing fibers protruded into the chondral part of the construct over which two layers of a thiol-ene cross-linkable hyaluronic acid/poly(glycidol) hybrid hydrogel (HA-SH/P(AGE-Co-G)) were fabricated. The top layer contained Articular Cartilage Progenitor Cells (ACPCS) derived from the superficial layer of native cartilage tissue, the bottom layer contained mesenchymal stromal cells (MSCs). The chondral part of control constructs were homogeneously filled with MSCs. After six months in vivo, microtomography revealed significant bone growth into the anchor. Histologically, there was only limited production of cartilage-like tissue (despite persistency of hydrogel) both in zonal and non-zonal constructs. There were no differences in histological scoring; however, the repair tissue was significantly stiffer in defects repaired with zonal constructs. The sub-optimal quality of the repair tissue may be related to several factors, including early loss of implanted cells, or inappropriate degradation rate of the hydrogel. Nonetheless, this approach may be promising and research into further tailoring of biomaterials and of construct characteristics seems warranted. 


\section{INTRODUCTION}

Osteochondral lesions, i.e. those involving both the articular cartilage and subchondral bone, may lead to tissue degeneration that can progress into osteoarthritis (OA) [1]. In clinics, a commonly applied strategy for (osteo)chondral repair relies on the recruitment of native repair cells through microfracture [2]. More sophisticated techniques include different forms of mosaicplasty [3] and implantation of osteochondral allografts [4]. Clinical trials have also used various commercially available scaffolds $[5,6]$, with or without cells.

However, these strategies are often associated with significant limitations, like donor site morbidity and high costs [7] and all result in insufficient functional repair [8].

The failure to achieve successful chondral repair may be due to the avascular nature of articular cartilage [9]. Recent research has focused on the generation of living personalized prostheses for joint repair through biofabrication techniques [10, 11], however, the resulting repair tissue still lacks the hierarchical, collagen structure typical of healthy native articular cartilage [12]. This results in formation of repair tissue that is biomechanically inferior, contributing to failure of function in the long-term [13]. Restoration of the bone part in osteochondral repair is less challenging, because of the innate repair response of bone [14], which is a well vascularized tissue. This discrepancy in intrinsic healing capacities between bone and cartilage makes the production of constructs for osteochondral defect repair a complex task, as it requires a highly specialized multiphasic scaffold to mimic the functional characteristics of the native osteochondral unit [15].

Tissue engineering techniques have the potential, through the use of multiple materials and cell types, to further approximate the zonal architecture of the native tissue $[16,17]$. To achieve this, appropriate choices need to be made with respect to both the biomaterials and the cells that are used. Hydrogels are considered promising biomaterials for cartilage repair because of their intrinsic hydrated nature. Also, they can be functionalized by incorporation of chemical cues, providing them a high degree of versatility for biofabrication applications, including replication of spatially organized constructs $[11,18]$. However, a definite drawback is that their stiffness is very limited, making them unsuitable to recreate the mechanical characteristics of 
the native tissue on their own [14]. A key challenge for successful regeneration is the choice of the biomaterials used to build a bio-inspired multiphasic zonally-organized construct [19]. In such constructs, hydrogels based on synthetic polymers, such as polyglycidols-based (PG) polymers [20] combined with a thiol-functionalized hyaluronic acid (HA-SH) [20], may offer the possibility to provide a cell-friendly environment while still permitting control of the chemical and physical properties [21].

To increase chances of success, it is also possible to improve the biomechanical performance of hydrogels by combining biomaterials with fiber reinforcements, although fiber thickness and deposition patterns have different effects on biomechanics [22], hence this principle was also adapted for this study. The combination of biomaterials and fiber reinforcement however, may be insufficient, as the regeneration is also driven by cells [23], partially through their interaction upon implantation with the host environment [24]. There is, however, still debate on which cell type may give optimal results for regenerative purposes [21, 25].

To assess if the appropriate choices have been made, a pre-requisite for eventual clinical application is the long-term evaluation in a reliable large animal model [26]. The equine model has often been described as a suitable option for this purpose, as the equine stifle has characteristics that resemble those of the human knee, and an equally, possibly more challenging mechanical environment [26, 27].

Chondrocytes have been used with some success in the equine model [28, 29], as have bone-marrow derived stem cells (MSCs), which, however, showed limited improvement in repair compared to control treatments in the longterm [30], and are known to exhibit a hypertrophic phenotype under chondrogenic induction [31]. A relatively unexplored cell population identified in the superficial layer of cartilage, known as Articular Cartilage Progenitor Cells (ACPCs) [32], may be more suitable than MSCs [33]. These cells show multipotent capacity for differentiation, as do MSCs, and in vitro, these cells synthesize collagen type II and aggrecan, but no collagen type $X$, marker of hypertrophic chondrocytes [34]. These cells also lack RUNX2, a master transcription factor for osteogenic differentiation observed during endochondral ossification [35]. Additionally, ACPCs harvested from the equine articular cartilage have been thoroughly characterised. In particular, gene expression analysis showed positivity of ACPCs for CD73, CD90, and CD105, 
negativity for hematopoietic marker CD34 and leukocyte marker CD45. ACPCs were also shown to be positive for CD29, CD44, CD49, CD106 and CD166 [23].

Furthermore, when embedded in a gelatin-based hydrogel, ACPCs were found to express PRG4, a gene that encodes production of lubricin in the superficial layer of cartilage [36], indicating that these cells represent a promising cell source for cartilage repair, and may instruct repair cells to develop a superficial zone phenotype [37] making them great candidates for attempting to recapitulate the zonal features of native cartilage. The principle of differences in spatial distribution to enhance chondral formation has been previously investigated with different cell sources such as chondrocytes [38], MSCs and ACPCs [23]. In the study by Levato et al. in particular, the in vitro combination of the two latter cell types appeared the most promising [23].

Therefore, here we investigate the performance of a multi-composite implant for the repair of osteochondral defects, focusing on the possible effect of a zonal distribution of the cellular component. A composite construct with zonal cell distribution was developed utilizing MSCs and ACPCs. The basic construct consisted of a thiol-ene cross-linkable hyaluronic acid/poly(glycidol) hybrid hydrogel (HA-SH/P(AGE-Co-G)) [20] and a 3D-printed poly(( $\varepsilon$ caprolactone) (PCL) osteochondral anchor previously tested for fixation [39] in the equine model. We evaluate the impact of a zonal configuration against a non-zonal configuration in vitro and in vivo in a long-term equine model for cartilage repair, as well as the long-term effects of the anchoring scaffold for bone repair.

\section{MATERIALS AND METHODS}

\section{Isolation and culture of cells}

\section{Cell expansion for in vitro experiments}

Equine MSCs and ACPCs were isolated as described by Levato et al. from two different donors (one for cell type) [24]. These cells were used for three independent experiments, in which they were cultured up to passage 4 in expansion medium. For MSCs this was DMEM high glucose $4.5 \mathrm{~g} \mathrm{l}^{-1}$ (SigmaAldrich, St. Louis, MO, USA), supplemented with 10 \% FCS (Thermo Fisher, Waltham, USA), $1 \mathrm{ng} \mathrm{ml}^{-1}$ bFGF (Recombinant Human FGF-basic/145aa, BioLegend, London, UK) and $1 \% \mathrm{P} / \mathrm{S}\left(100 \mathrm{U} \mathrm{ml}^{-1}\right.$ penicillin, $0.1 \mathrm{mg} \mathrm{ml}^{-1}$ 
streptomycin, Thermo Fisher, Waltham, USA). For ACPCs: DMEM high glucose $4.5 \mathrm{~g} \mathrm{l}^{-1}$, supplemented with $10 \%$ FCS, $1 \%$ MEM NEAA (Thermo Fisher, Waltham, USA), $0.2 \mathrm{mM}$ L-ascorbic acid 2-phosphate (Sigma-Aldrich, St. Louis, $\mathrm{MO}, \mathrm{USA}), 5 \mathrm{ng} \mathrm{ml}^{-1} \mathrm{bFGF}$ and $1 \% \mathrm{P} / \mathrm{S}$.

Cell encapsulation in HA-SH/P(AGE-Co-G) hydrogels for in vitro experiments

For hydrogel preparation, HA-SH (5 wt.\%), P(AGE-co-/G) (5 wt.\%), and the photoinitiator 12959 (0.05 wt.\%, BASF, Ludwigshafen, Germany) were dissolved in PBS (Thermo Fisher, Waltham, USA) [20]. The $\mathrm{pH}$ of the slightly acidic solution was neutralized using $5 \mathrm{M} \mathrm{NaOH}$ (Sigma-Aldrich, St. Louis, MO, USA). Subsequently, MSCs or ACPCs at passage 4 were suspended in the precursor solution $\left(20 \times 10^{6}\right.$ cells per $\left.\mathrm{mL}\right)$, and the cell suspension was cast in cylindrical silicone molds with a diameter of $6 \mathrm{~mm}$. Constructs containing either MSCs or ACPCs alone had a volume of $55 \mu \mathrm{l}$ and were crosslinked via UV irradiation at $365 \mathrm{~nm}$ for $10 \mathrm{~min}$. For zonal constructs, $35 \mu \mathrm{MSC}$ suspension was first irradiated for $5 \mathrm{~min}$; then, $20 \mu \mathrm{ACPC}$ suspension was pipetted on top and irradiated again for $5 \mathrm{~min}$ to complete crosslinking. Pure hydrogel constructs (without PCL) were used for in vitro experiments. Constructs were cultured for $28 \mathrm{~d}$ in chondrogenic differentiation medium (DMEM high glucose, supplemented with $1 \%$ v/v ITS + premix (Corning, NY, USA), 0.2 mM L-ascorbic acid-2-phosphate, 100 nM dexamethasone (Sigma-Aldrich, St. Louis, MO, USA), $10 \mathrm{ng} \mathrm{ml}^{-1}$ TGF- $\beta 1$ (BioLegend, London, UK), 1 \% P/S). Per construct, $1 \mathrm{ml}$ medium was used and refreshed every 2-3 d. For in vitro evaluation, three independent experiments were conducted. For each experiment, the number of samples per group was $(n)$ equals 3 .

\section{Generation of constructs for in vivo study}

\section{Osteochondral constructs}

- Osteochondral anchor

Osteochondral anchors were fabricated as previously described in Mancini et al. [39]. Briefly, a screw-based extruder on a 3DDiscovery printer (regenHU SA, Switzerland) was used to 3D print GMP-grade PCL (Purasorb ${ }^{\circledR}$ PC 12, Corbion, The Netherlands). The osteochondral plug was designed using BioCAD software (regenHU SA) as a cylinder with $6 \mathrm{~mm}$ diameter, featuring a squaregrid scaffold structure with six zones with different porosities. The design was 


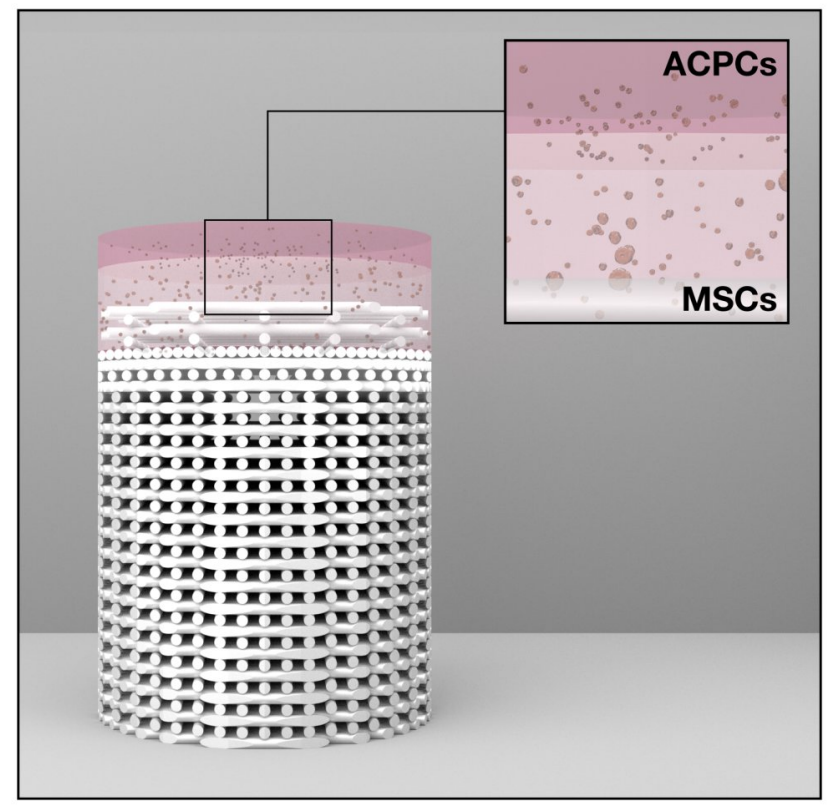

Figure 1. HA-SH/P(AGE-Co-G) hydrogel/cells mixtures were cast in a layer-wise fashion on the 3Dprinted PCL osteochondral scaffold. To produce the zonal constructs, the first layer of the chondral portion of the constructs was seeded with equine MSCs (35 $\mu l, 20 \times 10^{6}$ cells $\mathrm{ml}^{-1}$ of hydrogel), the second with chondroprogenitor cells (ACPCs, $20 \mu \mathrm{l}, 20 \times 10^{6} \mathrm{cells} \mathrm{ml}^{-1}$ of hydrogel).

replicated with decreasing porosity to mimic transition from trabecular to subchondral bone, resulting in a virtually closed interface between the chondral and osteal compartments, and a chondral fiber reinforcement to enhance fixation of biomaterials and increase biomechanical resistance [39]. The scaffold also allowed for press-fit fixation of the implant.

\section{- Cell encapsulation in HA-SH/P(AGE-Co-G) hydrogels}

Cell encapsulation was, in principle, executed as described above, using cells from the same donors (section 2.1). The HA-SH/P(AGE-co-G) hydrogel/cell mixture was cast in a layer-wise fashion on top of the 3D-printed PCL osteochondral scaffold. Zonal architecture was determined by subsequent casting of two hydrogel layers with different cell types, while non-zonal constructs were constituted of a single layer of hydrogel laden with a single cell-type. Specifically, for the chondral portion of the constructs a single layer was cast with $55 \mu \mathrm{l}$ of hydrogel mixed with equine MSCs $\left(20 \times 10^{6}\right.$ cells $\mathrm{ml}^{-1}$ of hydrogel) for the non-zonal constructs. For the zonal constructs, two hydrogel layers seeded with equine MSCs ( $35 \mu \mathrm{l}, 20 \times 10^{6}$ cells $\mathrm{ml}^{-1}$ of hydrogel) and with 
chondroprogenitor cells $\left(20 \mu \mathrm{l}, 20 \times 10^{6}\right.$ cells $\mathrm{ml}^{-1}$ of hydrogel) were consecutively cast on the osteochondral anchor (Fig. 1). Constructs were stored in differentiation medium (see paragraph on cell encapsulation for in vitro experiments) overnight and soaked with DMEM high glucose with no additions before implantation.

\section{Evaluation of chondrogenesis in vitro}

\section{Biochemical analysis}

After $28 \mathrm{~d}$, constructs $(n=3)$ were harvested and DNA, GAG and hydroxyproline content was measured [40]. Briefly, a TissueLyser (Quiagen, Hilden, Germany) was used to homogenize samples at $25 \mathrm{~Hz}$ for $5 \mathrm{~min}$ and afterwards samples were digested in a papain solution $\left(3 \mathrm{U} \mathrm{ml}^{-1}\right)$ for $16 \mathrm{~h}$ at $60^{\circ} \mathrm{C}$. DNA content was measured fluorometrically using Hoechst 33258 DNA intercalating dye (Ex: $360 \mathrm{~nm}, \mathrm{Em}$ : $460 \mathrm{~nm}$ ), with salmon testes DNA as a standard [41]. GAG content was determined by DMMB assay at $525 \mathrm{~nm}$, using bovine chondroitin sulfate as a standard [42]. The amount of hydroxyproline was measured after acid hydrolysis and reaction with $D A B$ and chloramine $T$ at $560 \mathrm{~nm}$, with L-hydroxyproline as a standard. Collagen content was determined based on a hydroxyproline to collagen ratio of 1:10 [43, 44].

\section{Histology and immunohistochemistry}

Constructs were first fixed in 3.7\% PBS-buffered formalin overnight and then, after dehydration, embedded in paraffin, and paraffin sections, $1 \mu \mathrm{m}$ thick, were obtained. For histological analysis of GAG deposited in the constructs, sections were stained with Weigert's hematoxylin, fast green and safranin- $O$ [45]. For immunohistochemical analyses, antigen retrieval was performed. For aggrecan, collagen type I and collagen type II sections were treated consecutively with pronase (30 $\mathrm{min})$ and hyaluronidase (30 $\mathrm{min})$. Sections were blocked with $1 \%$ BSA in PBS for 60 min. For visualization, sections were stained with respective primary antibodies: anti-aggrecan (1:300, 969D4D11/ AHP0022, Thermo Fisher, Waltham, USA), anti-collagen I (1:200, ab34710, Abcam, Cambridge, UK) and anti-collagen II (1:200, ab34712, Abcam, Cambridge, UK). For immunofluorescence staining, the secondary antibody Alexa Fluor 488 goat anti-rabbit IgG (1:400, 111-545-003, Jackson Immuno Research, Ely, UK) was used. For immunohistochemical staining with 3,3'- 
diaminobenzidine (DAB) as chromogen, the ultra streptavidin HRP detection kit (USA) (Biolegend, San Diego, USA) was used.

\section{Long term evaluation of constructs in the equine model}

A study with eight Shetland ponies was performed comparing a zonal versus a non-zonal configuration in the same animal. Zonal and non-zonal constructs were randomly implanted in either the right or left stifle joint. The study was approved by the local Ethics Committee for Animal Experimentation of Utrecht University (CCD n ${ }^{\circ}$ AVD108002015307) and was performed in accordance with the Institutional Guidelines on the Use of Laboratory Animals in compliance with the Dutch Act on Animal Experimentation.

The ponies ( $n=8$, age 4-10 years, weight $167-187 \mathrm{~kg}$ ) were free of lameness and without any clinical or radiographic evidence of acute or chronic injuries. They were housed together and fed a standard maintenance ration of concentrate with hay ad libitum and free access to water.

General anesthesia was induced with midazolam and ketamine intravenously (0.06 and $2.2 \mathrm{mg} \mathrm{kg}^{-1}$, respectively), after premedication with detomidine and morphine (10 $\mathrm{mcg} \mathrm{kg}^{-1}$ and $0.1 \mathrm{mg} \mathrm{kg}^{-1}$, respectively); anesthesia was maintained with isoflurane (1.1-1.5\% end tida in oxygen) with intravenous continuous rate infusion (CRI) of ketamine $\left(0.5 \mathrm{mg} \mathrm{kg}^{-1} \mathrm{hr}^{-1}\right)$ and detomidine (10 $\mathrm{mcg} \mathrm{kg}^{-1} \mathrm{hr}^{-1}$ ). Furthermore, before implantation surgery all ponies received an epidural injection with morphine $0.1 \mathrm{mg} \mathrm{kg}^{-1}$ bwt in $8 \mathrm{ml}$ of saline solution just before induction of anesthesia.

The medial trochlear ridge of the femoropatellar joint was exposed by arthrotomy through a subpatellar approach between the medial and middle patellar ligament; in each joint, an osteochondral defect with a diameter of 6 $\mathrm{mm}$ and a depth of $7.5 \mathrm{~mm}$ was created using a custom-made surgical drill and drill guide. After the scaffold was placed (press-fit), the wounds were closed in 4 layers using resorbable suture material (VicrylTM, Ethicon, US, LCC) and MonocrylTM (Ethicon, US, LCC) for the skin. Stents were placed over the wound for protection for the first $48 \mathrm{~h}$ and subsequently removed. Full weight bearing was allowed after recovery from anesthesia. Post-operatively, the ponies received NSAIDS (meloxicam, $0.6 \mathrm{mg} \mathrm{kg}^{-1}, \mathrm{PO}, \mathrm{BID}-\mathrm{SID}$ ) up to $7 \mathrm{~d}$ and tramadol (5 mg kg-1, PO, BID-SID) up to $3 \mathrm{~d}$ postoperatively. Prophylactically, antibiotics were administered peri-operatively (ampicillin (10-15 $\mathrm{mg} \mathrm{kg}^{-1}$, IV), while 
procaine penicillin (20 mg kg-1, IM) was administered once after surgery.

\section{Post-operative evaluation}

Orthopedic examinations were performed before surgery (baseline), and 2, 4 and 6 months after defect creation, and included objective gait analysis with a wireless networked inertial measurement system motion capture-based system (Qhorse, Qualisys AB, Göteborg, Sweden) [46]. Motion of the pelvis and protraction/retraction angle of the hindlimbs were assessed as an outcome parameter for lameness.

Second-look arthroscopy was performed at 4 and 6 months after defect creation and subsequent repair, and assessed repair tissue for attachment to surrounding articular cartilage, firmness, degree of filling and macroscopic appearance, using the Oswestry Arthroscopy Score [47].

After 6 months, animals were sedated with detomidine $\left(10 \mathrm{mcg} \mathrm{kg}^{-1}\right)$ and subsequently euthanized by administration of pentobarbital (50 mg kg-1 of body weight). Gross assessment of the medial trochlear ridge after euthanasia consisted of evaluating the volume of the repair tissue, integration of the margins of the repair tissue with the surrounding native tissue and surface quality. After this, the entire osteochondral area of the medial femoral ridge containing the constructs was harvested for analysis using a surgical bone saw.

\section{Biomechanical analysis of repair tissue}

A displacement-controlled nano indenter (Piuma, Netherlands) including a controller, an optical fiber and a spherical probe (Optics, Netherlands) was used to obtain force-displacement curves on the surface of the repair tissue. The probe is attached to a spring cantilever, which is connected to the end of the optical fiber allowing to measure the cantilever deflection. A probe with radius $X(54 \mu \mathrm{m})$ and cantilever stiffness $Y\left(84.3 \mathrm{~N} \mathrm{~m}^{-1}\right)$ was used to perform an indentation protocol comprising an indentation phase to a depth of $18 \mu \mathrm{m}$ for $1 \mathrm{~s}$ in the loading phase, followed by $7 \mathrm{~s}$ holding time, and an unloading time of $20 \mathrm{~s}$ [48]. A minimum of 3 measurements were taken at different locations within the defect, and then values were averaged.

\section{Evaluation of bone repair}

Each defect was scanned in a micro-CT scanner ( $\mu$-CT 80, Scanco Medical AG, Switzerland) at a resolution of $20 \mu \mathrm{m}$. The acquisition parameters were set to a 
voltage of $70 \mathrm{kVp}$, an intensity of $114 \mu \mathrm{A}$, and an integration time of $300 \mathrm{~ms}$. Subsequently, the acquired images were processed by first applying a Gaussian filter (sigma $=2$, support $=0.8$ voxel) and then segmentation. The images obtained were processed with BoneJ (a free plugin of ImageJ) to obtain the bone volume fraction (BV/TV) in the defect.

\section{- Parenting DNA analysis}

Full-thickness biopsies of repair tissue were obtained post-mortem and frozen at $-20^{\circ} \mathrm{C}$. DNA was extracted using the Kleargene spin plate according to the manufacturer's instructions (LGC, UK). The PCR of 17 equine STR markers was performed according to van de Goor et al. [49] by VHL Genetics, Wageningen, the Netherlands. Markers were compared between donor cells and host cells to detect possible presence of donor cell DNA at 6 months.

\section{Histology}

The osteochondral tissue blocks containing the defects were fixated in $4 \%$ formalin, decalcified with Formical-2000 (EDTA/formic acid; Decal Chemical Corporation, Tallman, NY) for $14 \mathrm{~d}$, dehydrated through a graded ethanol series, cleared in xylene and then embedded in paraffin. Samples were sectioned into $5 \mu \mathrm{m}$ slices and stained with hematoxylin and eosin (HE) and Safranin-O. Repair was assessed by a modified O'Driscoll scoring and ICRS scoring [50], and cell infiltration evaluated using an Olympus BX51 light microscope. Samples were also stained with picrosirius red and analyzed with polarized light microscopy for visualization of collagen fibril orientation. Immunohistochemical staining was performed for collagen type-I and type-II.

\section{Statistical analysis}

Statistical analysis for in vitro evaluation was performed using Graph Pad Prism 6.0 (GraphPad Software, La Jolla, USA) by two-way ANOVA with a Tukey 's post-hoc test. Differences between experimental groups were considered statistically significant at $p<0.05$.

Statistical analysis for in vivo study was performed by means of the statistical package SPSS (version 25.0, IBM, Armonk, NY), using one-way ANOVA, comparing zonal and non-zonal constructs (statistical significance set at $p<0.05)$.

Statistical analysis for objective gait analysis was performed in R [51] using 
linear mixed models with horse used as a random effect and time as a fixed effect. Significance was set at $p<0.05$ and correction for multiple comparison was done using the false discovery rate method.

\section{RESULTS}

\section{In vitro study}

\section{Biochemical analysis}

Within $28 \mathrm{~d}$ of in vitro culture, ACPCs and MSCs alone, as well as combined in zonal constructs were able to produce distinct amounts of GAGs within HA$\mathrm{SH} / \mathrm{P}(\mathrm{AGE}-\mathrm{Co}-\mathrm{G})$ hydrogels. MSCs produced significantly more GAGs than ACPCs (Fig. 2A, B). In line, collagen production was also observed within all constructs, with higher collagen content for MSCs compared to ACPCs (Fig. 2C,
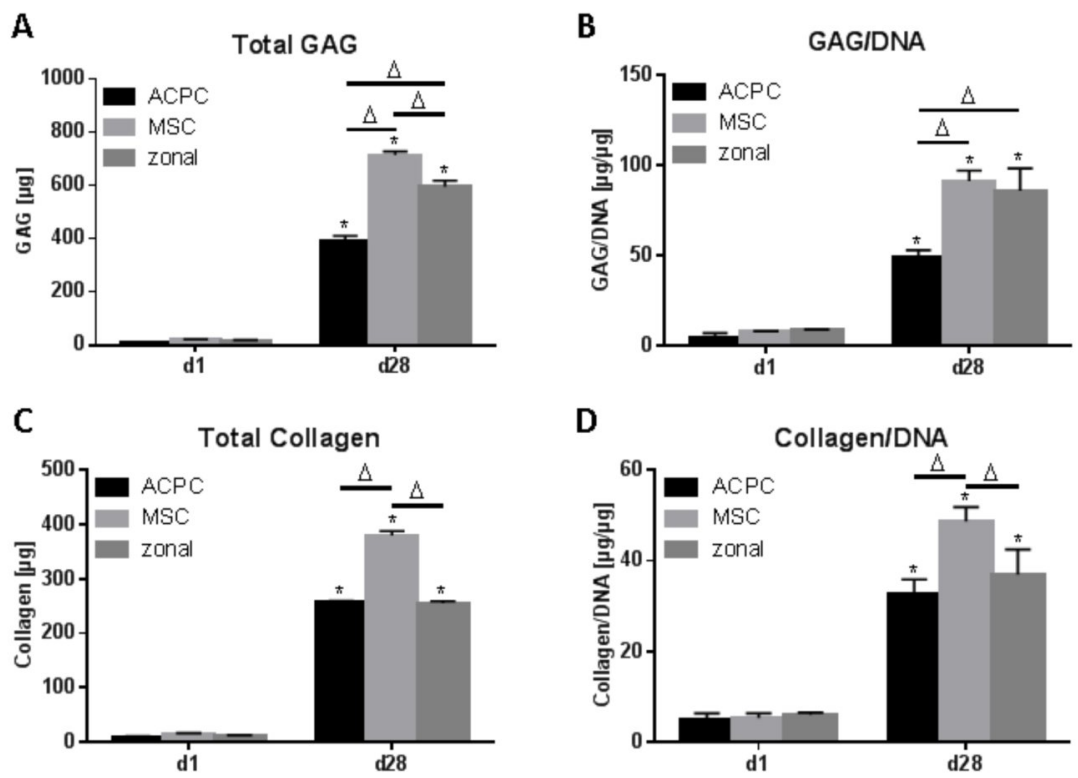

Figure 2. Biochemical analysis of ECM production in HA-SH/P(AGE-CO-G) hydrogel constructs, seeded with $20.0 \times 10^{6} \mathrm{cells} \mathrm{m}^{-1}$, after 1 and $28 \mathrm{~d}$ of chondrogenic differentiation. (A) Production of total GAG (GAG/construct); (B) GAG normalized to DNA (GAG/DNA); (C) production of total collagen (collagen/construct); (D) collagen normalized to DNA (collagen/DNA). Three independent experiments with $n=3$ biological replicates each were performed. Data are presented as means \pm standard deviation. $(*)$ indicates statistically significant differences between a d28 value and the corresponding $d 1$ value of the same group $(p<0.05)$. ( $\Delta)$ indicates statistically significant differences between groups $(p<0.05)$. 
D). Zonal layering of ACPCs and MSCs did not result in higher ECM levels compared to non-zonal constructs.

\section{Histological analysis}

GAG deposition was observed in all constructs. In line with the biochemical analysis, MSC-laden constructs showed more intense GAG staining than ACPCladen constructs. This difference was also reflected in the zonal constructs, with stronger staining in the MSC-containing bottom part. In all constructs, GAGs were mainly observed in pericellular regions, i.e., not homogeneously distributed throughout the entire construct (Fig. 3A). Similar observations were made in the immunofluorescence and immunohistochemical stainings for aggrecan and collagen types I and II (Fig. 3B-D; Figs. S1, S2, Supporting Information). High magnification of hydrogel constructs showed matrix intra-

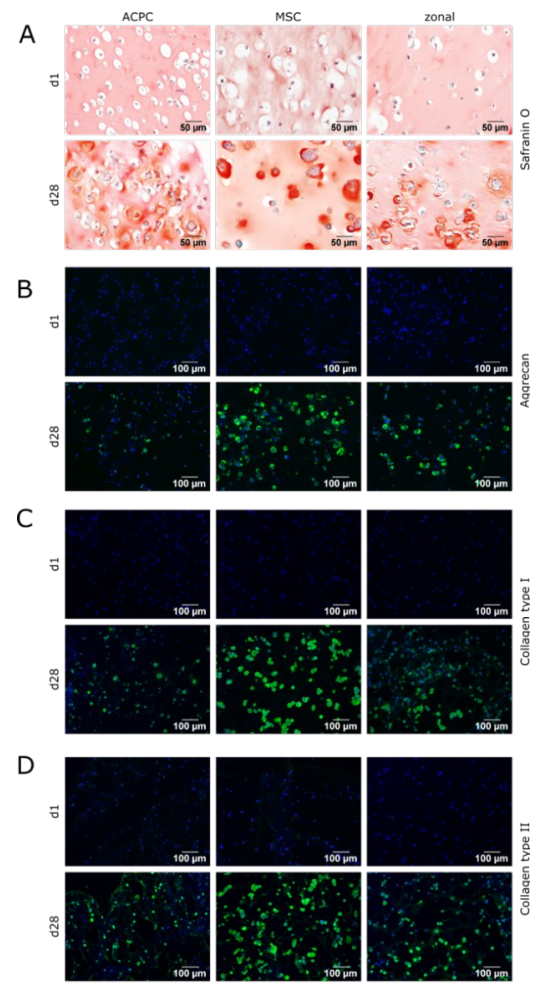

Figure 3. Histological and immunofluorescence stainings of (HA-SH/P(AGE-CO-G)) hydrogel constructs, seeded with $20 \times 10^{6}$ cells $\mathrm{ml}^{-1}$, after 1 and $28 \mathrm{~d}$ of chondrogenic differentiation. (A) Safranin $O$ staining for GAG. Immunofluorescence stainings for (B) aggrecan, (C) collagen type I and $(D)$ collagen type II. Three independent experiments with $n=3$ biological replicates each were performed. Representative data from one experiment is shown. Scale bars represent $100 \mu \mathrm{m}$. 
and mainly pericellularly distributed around the cells (Fig. S3, Supporting Information). In general, it was observed that ACPCs preferentially produced extracellular matrix in the outer areas of the constructs, whereas MSCs produced matrix within the whole construct (Fig. S2, Supporting Information).

\section{Long term evaluation of constructs in the equine model}

\section{Post-operative evaluation}

Clinical examinations after surgery did not reveal evident signs of lameness or joint effusions. All wounds healed by primary intention without complications. Objective gait analysis was performed with the motion capturebased QHorse system. For both hindlimbs, maximal protraction angle was reduced after surgery and remained reduced until the last time point $5 \mathrm{deg}$ reduction, $p<0.001$, Fig. S5A) Maximal retraction of the hindlimbs was initially reduced after surgery and increased over time, when compared to baseline ( $2.5 \mathrm{deg}, p<0.001$. Fig. S5B). Pelvis rotation along the horizontal plane (yaw) was reduced after surgery and increased at the last time point when compared to baseline ( $2.4 \mathrm{deg}, p=0.033$, Fig. S5C). Vertical motion symmetry of the pelvis in the sagittal plane reduced after surgery $(1.2 \mathrm{~mm}, p=0.004$, Fig. S5D).

At 4 months after implantation, repair tissue filled all defects and appeared well integrated with the surrounding cartilage tissue (Fig. 4A, C). The aspect of the repair tissue changed from transparent to more whiteish over time (Fig. 4B, D). The semi-quantitative Oswestry scoring [47] was not different in both groups and did not show significant changes between 4 and 6 months (Fig. 4E).

\section{Biomechanical analysis of the repair tissue}

The average compressive modulus of the repair tissue in the zonal group was $147.5 \pm 40.7 \mathrm{kPa}$. In the non-zonal constructs, this figure was significantly lower (96.9 $\pm 33.0 \mathrm{kPa}, p<0.05)$ (Fig. 5). Healthy cartilage from the same animals ranged at $495.9 \pm 174.0 \mathrm{kPa}$.

\section{Evaluation of bone repair}

The bone volume fraction was similar for both groups, averaging $26.7 \% \pm$ $5.6 \%$ for zonal and $25.7 \% \pm 3.4 \%$ for non-zonal groups (Fig. 6A). Micro-CT revealed good integration of the scaffold with the surrounding native tissue, 


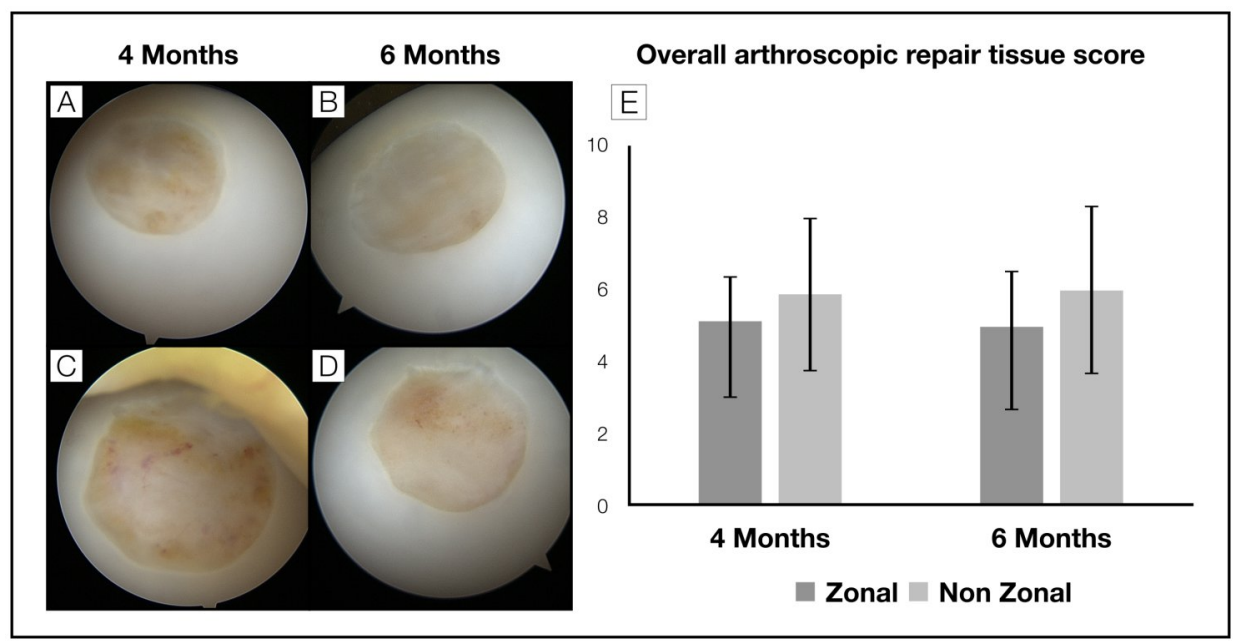

Figure 4. Arthroscopic images of representative defects at 4 and 6 months (top zonal, bottom non-zonal constructs). (A), (C) At 4 and 6 months, repair tissue filled all defects, appearing well integrated with the surrounding cartilage. (B), (D) Appearance of repair tissue changed from transparent to more whiteish over time. (E) Semi-quantitative scoring according to the oswestry arthroscopy score showed similar results for overall repair tissue quality in both groups, with no significant changes between 4 and 6 months.

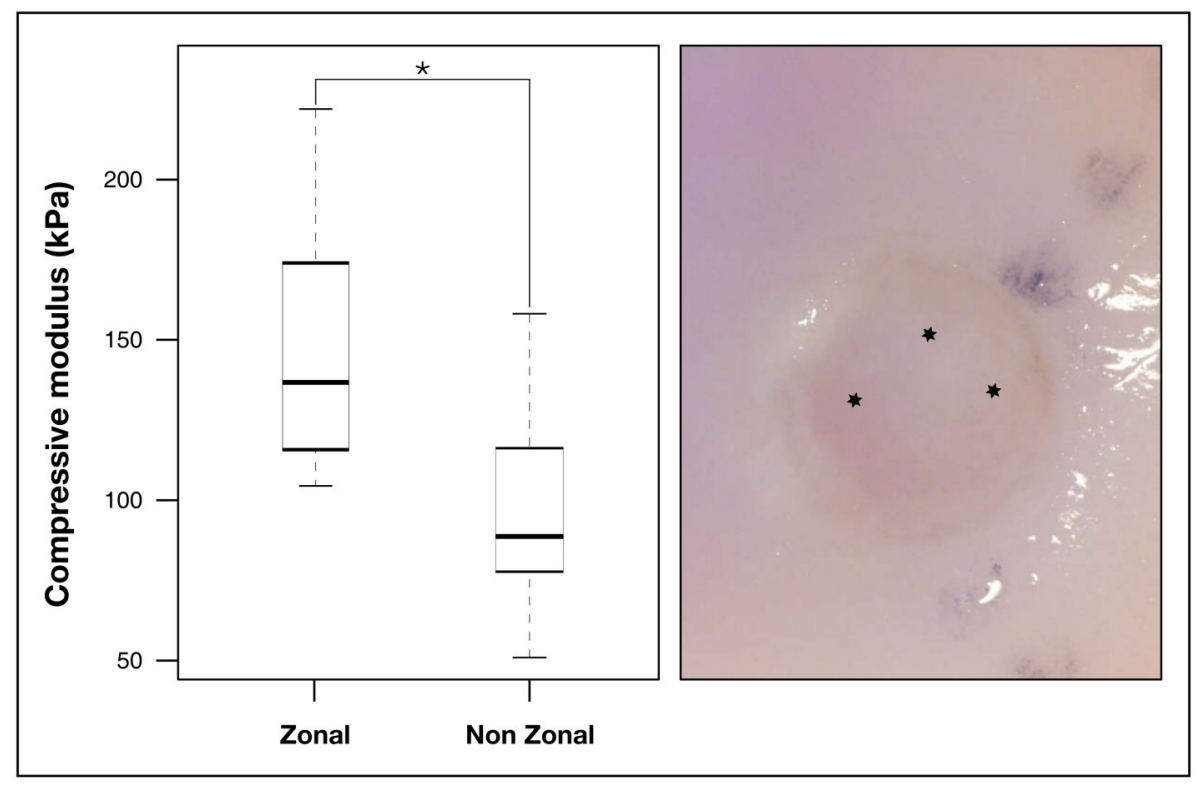

Figure 5. Biomechanical characterization of newly formed tissue filling the defects with microindentation. Average value of the compressive modulus in the zonal group was $147.5 \pm 40.7 \mathrm{kPa}$, while in the defects repaired with non-zonal constructs, the average compressive modulus was significantly lower $(96.9 \pm 33.0 \mathrm{kPa}, \mathrm{p}<0.05)$. A minimum of 3 measurements were performed in different areas of the defect for each animal ( $n=8$, right, black stars). 
and presence of mineralized tissue within the scaffold (Fig. 6B, C).

Polarized light microscopy showed presence of collagen fibers throughout the osteal portion of the scaffold (Fig. 7A, B).

Histology showed new tissue formation in construct pores with a bone-like structure (Fig. 7C, D), and presence of multinucleate cells along the PCL fibers (Fig. 7E, F).

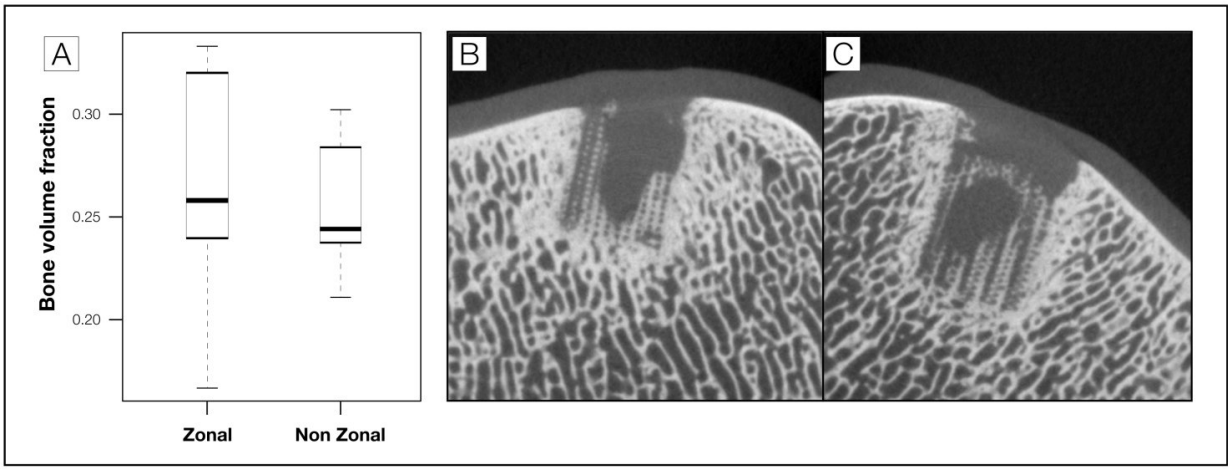

Figure 6. (A) Bone volume fraction (BV/TV) was similar for both groups, averaging $26.7 \% \pm 5.6 \%$ for zonal and $25.7 \% \pm 3.4 \%$ for non-zonal constructs $(n=8)$. ( $B$ zonal, $C$ non-zonal) Micro-CT images of representative samples showing integration of the scaffold with the surrounding native tissue, and production of new mineralized tissue within the scaffold.

\section{Evaluation of chondral repair tissue}

Six months after surgery all defects were filled with tissue with heterogeneous appearance, suggesting a varying degree of repair tissue production. Traces of hydrogel fragments were detected. Histological analysis showed that the defect was filled with repair tissue level to the surrounding tissue, and well-integrated with the native bone and cartilage (Fig. 8A, D). Immuno-histological staining characterized the tissue as prevalently fibrous (Fig. 8B, E) with scarce to none presence of collagen type II, independently of zonal or non-zonal configuration (Fig. 8C, F). Staining for collagen type I appeared consistently more intense in the zonal group. Semi-quantitative histological scoring of the repair tissue did not show significant differences between zonal and non-zonal constructs (Fig. 9).

Parenting of DNA showed no evidence of DNA presence from donor cells and only host cells were detected. 


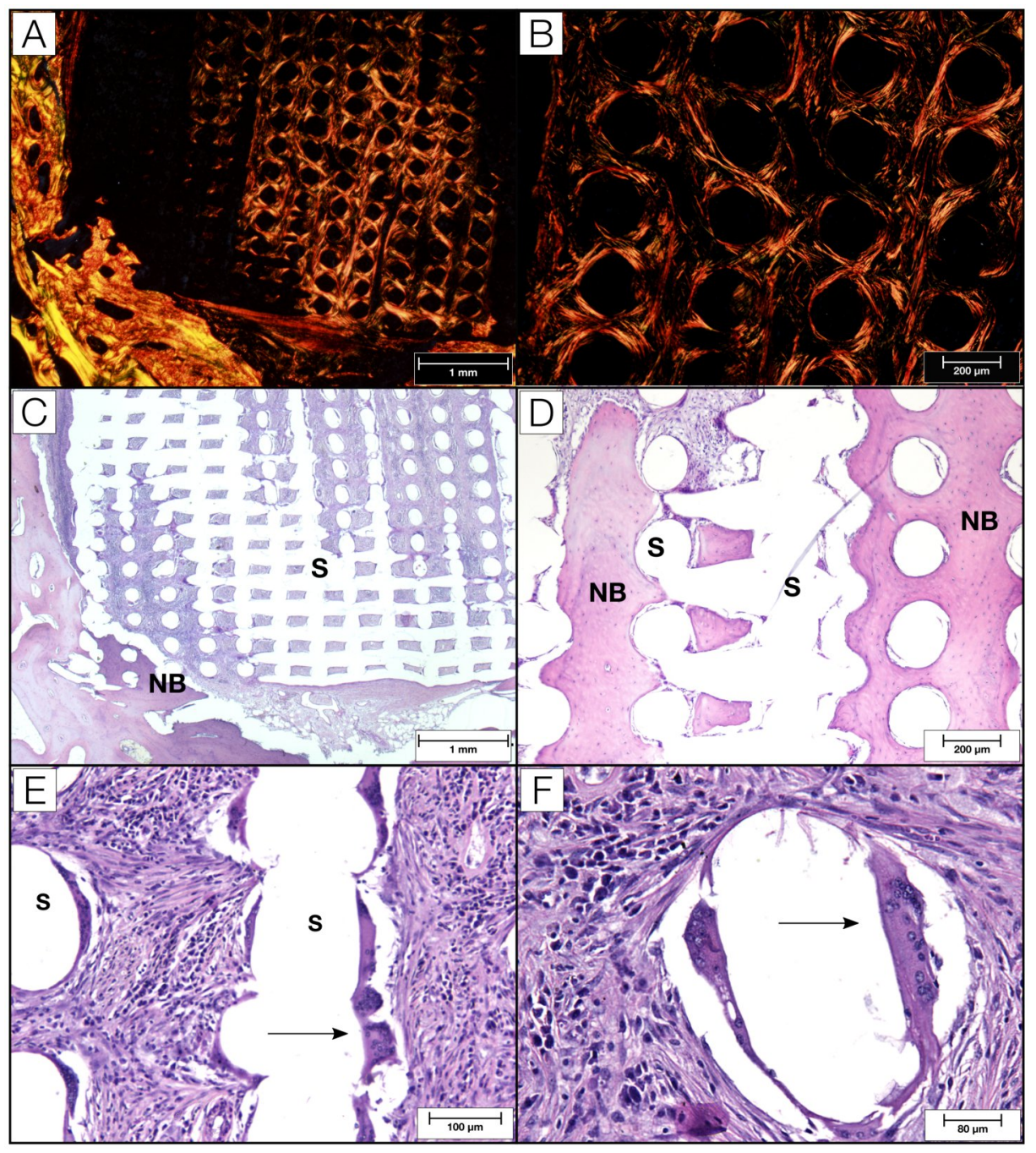

Figure 7. (A), (B) Polarized light microscopic histological images of the osteoal portion of the scaffold stained with picro-sirius red, showing presence of collagen fibers throughout. (C), (D) Hematoxylin eosin staining showed infiltration of the scaffold $(S$, empty space left after dissolution during histological processing) with new bone-like tissue (NB). (E), (F) At higher magnification lying along the PCL fibers of the scaffold multinucleated cells could be observed (black arrows). 


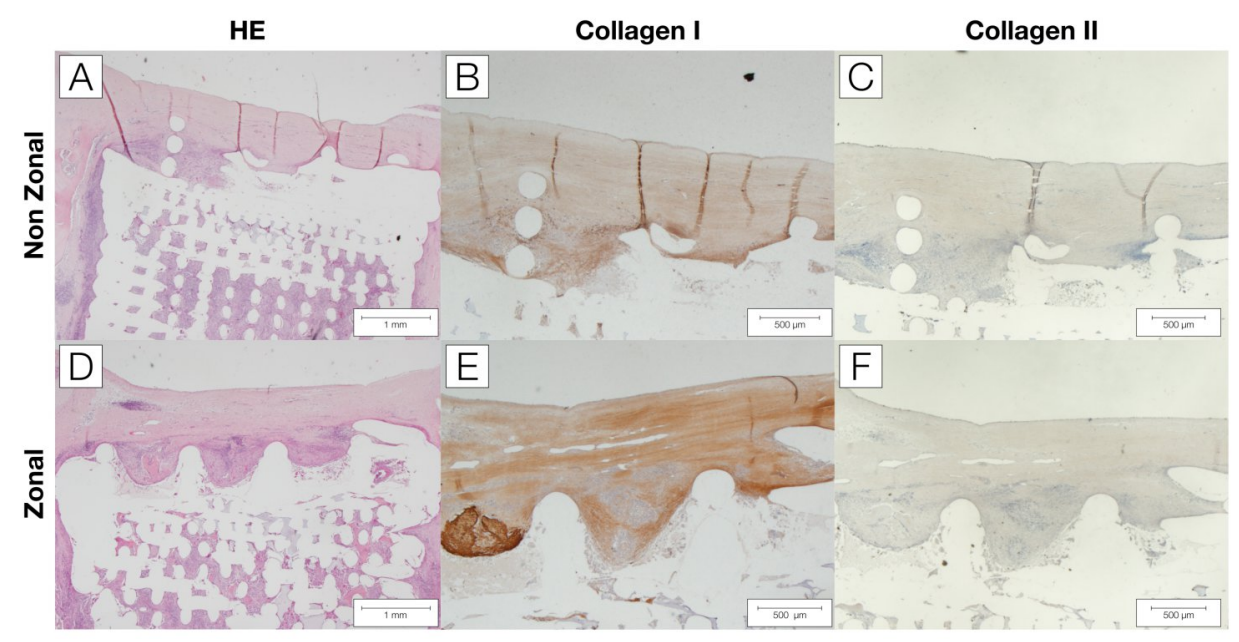

Figure 8. (A), (D) Histological sections stained with hematoxylin-eosin showing repair tissue level with the surrounding tissue, and integration with the native bone and cartilage. (B), (E) Immunohistological staining showing widespread production of collagen I, but scarce to none presence of collagen type II, independent of zonal or non- zonal configuration (C), (F).

\section{DISCUSSION}

This study is the first to report on the long-term outcome of the use of a cellseeded multi-composite fibre-reinforced hydrogel scaffold with a 3D printed anchor for fixation in the subchondral bone for the repair of artificially created osteochondral defects in an equine in vivo model, comparing a zonal versus a non-zonal configuration of the cartilage component. Overall, the results are similar to other longer term studies on cartilage repair in large animal models or in humans: clinically satisfying (at least for the duration of the study), a macroscopically reasonable filling of the defect, but histologically formation of fibrocartilage at best, characterized by low, if any GAG content, production of collagen type I rather than type II and biomechanical properties that fall far short of those of native tissue $[5,12,52,53]$. In the case of the current study the zonal constructs were significantly stiffer than the non-zonal constructs, but compressive stiffness was still a factor 6 or 7 less than that of native cartilage.

The combination of cell-seeded biomaterials with a 3D-printed scaffold is a versatile approach to address the challenge of osteochondral repair, enabling creation of mechanically competent scaffolds, while still allowing guidance of 


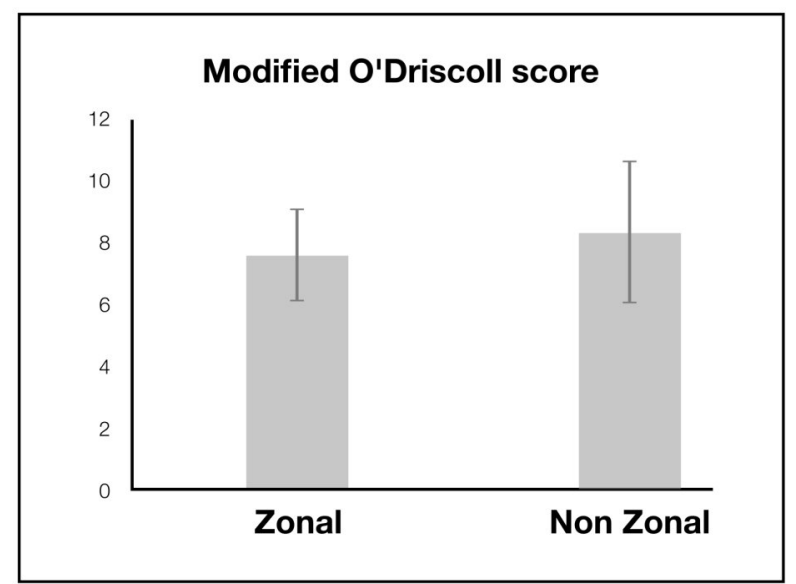

Figure 9: Microscopic scoring according to a modified O'Driscoll score did not show significant differences between the groups $(n=8)$.

regeneration based on cells and hydrogels [54]. The choice of cell type may play a determining role. Previous studies have shown that chondrocytes may suffer dedifferentiation during in vitro expansion $[55,56]$, contrarily to MSCs [57]. However, MSCs tend to differentiate terminally and thereby can cause hypertrophy and partial matrix calcification in the tissue [58]. Nevertheless, they are still utilized for cartilage regeneration, particularly in combination with other cell types [23]. ACPCs on the other hand, can be expanded in vitro without losing their chondrogenic potential [33], and have been shown to differentiate chondrogenically without hypertrophy or matrix calcification [35], rendering them a promising cell source for cartilage regeneration.

The choice of allogeneic cells for the execution of the in vivo experiments, originates from the rationale that use of allogeneic cells would allow execution of a single step procedure, avoiding complications and costs related to twostep procedures that are necessary when using autologous cells [59]. In this study, allogeneic equine MSCs and ACPCs from two separate donors were seeded into HA-SH/P(AGE-Co-G) hydrogels in non-zonal and zonal constructs. Equine MSCs have been cultured in vitro in HA-SH/P(AGE-co-G) hydrogel before and, when considering the different cultivation times, the levels of GAG/ DNA and collagen/DNA were in the same range as in the present study [20]. The combination of MSCs and ACPCs in zonal constructs has also been previously investigated, although in association with a different gelatin-based biomaterial (gelMA) [23]. In that study, a comparison of zonal co-cultures 
between ACPCs, MSCs, and chondrocytes showed that the combination of ACPCs in the upper layer and MSCs in the lower layer outperformed the other combinations. MSCs produced highest levels of GAG/DNA, followed by ACPCS and then chondrocytes [23].

This promising data inspired us to use the same combination of cell types in HA-SH/P(AGE-co-G) hydrogels. In the present study, MSCs outperformed ACPCs in non-zonal constructs as well. A possible reason for this could be that the hydrogel -as well as the differentiation medium- was initially developed for MSCs; therefore with suitable adjustments, it may be possible to increase in vitro matrix production by ACPCs. Matrix production and distribution in zonal constructs reflected those of non-zonal constructs of the respective cell type, with more GAG and collagen in the lower layer (MSCs) compared to the superficial layer (ACPCs). As this distribution is similar to native cartilage structure, this approach towards a zonal construct consisting of two different cell types seemed promising for further evaluation in the animal model.

Even when in vitro studies show encouraging results, translation of tissue engineering strategies to relevant large animal preclinical models remains a persisting challenge $[52,60,61]$. Nevertheless, the large animal models are an indispensable step in the evaluation of any therapy for osteochondral repair [62], and among them the equine model is considered as probably the most challenging large animal model for cartilage repair [26]. Advantages of this model include accessibility of the joint, that allows for monitoring the repair process in long-term studies [26], and the fact that cartilage biochemical composition and thickness [27] and (subchondral) bone density approximate that of humans [63]. Disadvantages include high costs and immediate loadbearing after surgery [52]. An important ethical advantage is that the horse, unlike the majority of experimental animals, is a frequent orthopedic patient itself with a clear clinical need for improved treatment of joint injuries, as it naturally suffers from joint trauma and osteochondral diseases, due to its regular use as an athletic and working animal [26].

It is recognized that, when addressing scientific questions in large animal in vivo studies, many complex variables come into play. Answering a scientific question in a satisfactory way is a fundamental prerequisite, however the design needs to accomplish this while respecting the 3R's principles. With the goal of reducing possible influences of multiple groups (which may provide more data but potentially introduce confounding factors) while maintaining a 
scientifically sound design, the authors opted for a direct, intra-individual leftright comparison to investigate the influence of a dual (zonal) distribution of cells on cartilage repair, eliminating the large inter-individual variations characteristic of outbred populations.

The simultaneous investigation of cell-free scaffolds, or empty defects may be valid and interesting, but not directly pertinent to the understanding of the influence of cell zonal distribution in cartilage repair. Cell-free scaffolds represent an alternative approach to cartilage repair with its own merits and drawbacks $[64,65]$. Similarly, while empty defects may represent a tempting control group, it is known from previous studies that defects of more than 4 $\mathrm{mm}$ of diameter will show poor regeneration, while requiring a higher number of animals [66]. The use of an untreated control defect thus becomes dispendious from an economic perspective, and is undesirable in terms of respect of the 3 Rs principles: the natural development of empty cartilage defects as little as $2 \mathrm{~mm}$ in diameter showed repair with fibrous, collagen I based tissue poor in proteoglycan content [66]. Previous authors have also opted for not using empty control defects, choosing for example a comparison with fibrin glue [30], which also presents some drawbacks in terms of fixation [39], or other repair strategies better suited for comparison to the aim of the study [28], including a direct comparison between two variations of a similar repair strategy [52], like in the case present here.

The use of multiple defects within the same joint would potentially allow for inclusion of multiple groups, while requiring a lower numbers of animals. Nevertheless, multiple larger defects within a single joint do feature the loss of proteoglycans in a relatively wide area around the defect, also affecting the surrounding healthy cartilage, and thus impacting on the repair potential of the evaluated treatments [67]. Apart from this, any additional defect will affect joint homeostasis and thus affect the conditions under which other defects in the joint must heal, making a multiple-defect approach methodologically questionable at least.

Based on earlier work on the fixation of constructs meant for the repair of focal cartilage lesions in the equine model [39], the choice was made for an osteochondral construct that could surgically be placed press-fit without need for further fixation. There are currently no satisfying techniques for fixating chondral constructs in the horse. In humans, fibrin glue is commonly used, however there are serious concerns with respect to its use in the horse, mainly 
because of immunological reasons [39], but also because of immediate postsurgical weight-bearing in the equine species. Biodegradable polydioxanone pins have been used with some success to secure cartilage flaps in horses affected by the developmental orthopaedic disease osteochondrosis [68], but these rely on the consistency of the native tissue (which is much less in hydrogel-based constructs) and would alter the architecture of 3D scaffolds [69]. The polycaprolactone osteochondral anchor that had been earlier designed and tested in short-term in vivo pilots [39] served its purpose well in this long-term in vivo study.

Previous equine studies testing biphasic osteochondral constructs commonly utilized either calcium phosphate (CaP) [52, 70, 71] or magnesium hydroxyapatite (Mg-HA) [5] for the osteal portion of the scaffold. This was the first long-term study in the horse that used polycaprolactone as the structural element of the osteal part of the scaffold. The material had, however, been used for reinforcement of scaffolds in other species [11, 72]. Polycaprolactone is a material that will degrade very slowly through hydrolysis (24-36 months) [73], and as expected no resorption was seen during the experimental period, differently from degradable CaP or HA-based constructs [52, 70, 71]. The PCL scaffold showed good integration with the surrounding bone with production of new mineralized tissue, in line with earlier reports [73]. Histology did not show presence of any inflammation, confirming the inertness of the material. Although the conclusion cannot be drawn definitively from the current data, it seems reasonable to presume that the scaffold will continue to slowly degrade and bone tissue will continue to be produced, likely leading to functional repair of the bone.

The performance of the cartilage portion of the implanted construct was less favourable than that of the osteal portion.

Interestingly however, clinically the animals did not display macroscopic signs of discomfort other than those expected from a surgical intervention; objective gait analysis showed that both the pelvis motion and limb movement seem to indicate a bilateral effect of the surgery. These results are compatible with a mild degree of bilateral lameness or disfunction although the effect may be very small and thus not clinically evident.

Although macroscopically the defects were for the major part neatly filled with repair tissue to the level of the original joint surface and no step defects of relevant size were seen, the tissue that was formed was virtually devoid of 
both glycosaminoglycans and collagen type II, whereas collagen type I was found in abundance. This type of repair tissue is similar to tissue commonly observed in repair of untreated osteochondral defects, or after surgical interventions such as micro-fracturing [12], and often represents the end stage of even more advanced therapies, such as $\mathrm{ACl}$ and $\mathrm{MACl}$ [12]. Apparently, the conditions in vivo were too far off from those that favoured chondrogenesis in the in vitro situation, where the two cell types as well as their combination showed both glycosaminoglycan and collagen type II production, be it at different rates.

It is important to state that the data presented here results from one possible zonal chondral approach and therefore its outcome should not be taken as representative for all possible zonal chondral strategies. Several factors may have played a role in obtaining these results and may offer the possibility to influence the outcome.

Firstly, DNA parenting showed that after 6 months no cells with DNA other than that of the host could be detected. This means that the seeded MSCs and ACPCs had all disappeared and had also failed in producing new generations of cells. From the experimental data it is not possible to determine when this happened, but evidently at 6 months post-implantation ongoing repair was realized solely by cells from the host. In that light, the fibrotic rather than hyaline character of the repair tissue may be unsurprising, as it is the type of tissue that is generated by intrinsic repair of articular damage [12]. The main effect seen in this study was the marginally -but significant- higher stiffness of the repair tissue in the sites treated with the zonal constructs. This was not associated with tangible histological differences within the zonal and nonzonal groups, other than a subjectively higher intensity of collagen type I staining. Although interesting, these findings cannot be said to represent better repair.

Secondly, the degradation rate of the hydrogel on top of the PCL scaffold where MSCs and/or ACPCs were encapsulated should be considered. Histology showed the scattered presence of some persistent hydrogel fragments after 6 months, leading to the conclusion that at least some of the material resisted for a relatively long period. Although very early degeneration is considered a negative factor for the success of regeneration [14], little is known about what the desirable degradation profile should be. This represents a great challenge in tailoring the degradation profile of any biomaterial destined for cartilage 
repair and it is, therefore, difficult to rate the rather long-lasting but heterogeneous degradation profile seen in the current study. There was histological evidence that early degeneration did not happen in this case, but it can be questioned to what extent some scattered fragments are enough to still have any effect on the chondrogenic capacity of cells and whether cues to promote chondrogenic performance should not remain present for much longer. What can be deduced here is that the degradation profile was apparently prolonged enough to permit good filling of the defect, level to its surroundings, and formation of a good connection at the scaffold-cartilage interface, which can be seen as encouraging, as this integration prevents further deterioration of the native tissue [34].

A third factor, that may be related to the former two, is the severe lack of stiffness of the cell-seeded hydrogel that was used on top of the PCL structure. Both the overall biomechanical properties of the implant and the biomechanical environment within the structure as perceived by the seeded cells, in our materials approach, are evidently different from those generated by the native collagen network. Fibre-reinforcement can substantially improve stiffness compared to hydrogels alone, although it should be noted that stiffer materials shield cells from experiencing mechanical stimuli which may then hinder matrix production [74]. It has however been demonstrated before, that a significant improvement in stiffness can be achieved through a specific architecture of the PCL structure -with a high-volume percentage of the composite scaffold filled by the hydrogel [22]. This would be an obvious next step for construct design, once the fabrication of scaffolds by combining different production processes needed to integrate this into the osteochondral anchor is possible.

It can be concluded that the PCL-based osteochondral construct used in this equine long-term study was easy to handle surgically, could be well fixated, and gave promising results with respect to the regenerative capacities of its osteal portion. The cartilage part failed to generate better repair tissue than that produced by natural intrinsic healing of osteochondral defects. This may be related to early loss of the seeded cells, the degradation rate of the hydrogel or the failure to recreate an environment that biomechanically resembles native articular cartilage, and most likely by a combination of these factors. Future approaches should focus on materials tailoring to mimic the biomechanical environment for the seeded cells without impeding them to 
produce extracellular matrix elements because of excessive biomaterial stiffness, and on optimizing the degradation profile of the hydrogel. Prematured multiphasic scaffolds might be a way to address some of the issues raised in this study. Another approach could be the stimulation of ingrowth of native cells combined with providing long-lasting regenerative cues to these, e.g. by controlled release from extracellular vesicles, rather than relying on implantation of allogeneic cells. In all approaches it is nevertheless expected that the structural element of cartilage will present the biggest challenge.

\section{ACKNOWLEDGEMENTS}

The authors would like to acknowledge T. Boeck for his expertise and help in preparation of hydrogels, $\mathrm{H}$. Weinans for his expertise on micro-CT analysis, $\mathrm{F}$. Abinzano for her help in preparation of the hydrogels, F. Serra Bragança for his expertise and help with objective gait analysis, J.C. de Grauw and J.P.A.M. van Loon and their team for taking care of anesthesia of the ponies and the complete 'equine cartilage repair team' for logistics around the surgeries.

The research leading to these results has received funding from the European Community's Seventh Framework Programme (FP7/2007- 2013) under grant agreement 309962 (HydroZONES), the Dutch Arthritis Foundation (ReumaNL, LLP-12 and LLP-22). Furthermore, this work was supported by the Deutsche Forschungsgemeinschaft (DFG, German Research Foundation) -Project number 326998133-TRR 225 (sub-project A02). 


\section{REFERENCES}

[1] M. Tamaddon, L. Wang, Z. Liu, C. Liu, Osteochondral tissue repair in osteoarthritic joints: clinical challenges and opportunities in tissue engineering, Bio-Design and Manufacturing 1(2) (2018) 101-114.

[2] J. Steadman, W. Rodkey, K. Briggs, Microfracture to treat full-thickness chondral defects: surgical technique, rehabilitation, and outcomes, J. Knee Surg. 15(3) (2002) 170-176.

[3] E. Solheim, J. Hegna, J. Øyen, O.K. Austgulen, T. Harlem, T. Strand, Osteochondral autografting (mosaicplasty) in articular cartilage defects in the knee: results at 5 to 9 years, Knee 17(1) (2010) 84-87.

[4] A.M. Torrie, W.W. Kesler, J. Elkin, R.A. Gallo, Osteochondral allograft, Curr. Rev. Musculoskelet. Med. 8(4) (2015) 413-422.

[5] E. Kon, A. Mutini, E. Arcangeli, M. Delcogliano, G. Filardo, N. Nicoli Aldini, D. Pressato, R. Quarto, S. Zaffagnini, M. Marcacci, Novel nanostructured scaffold for osteochondral regeneration: pilot study in horses, J. Tissue Eng. Regen. Med. 4(4) (2010) 300-308.

[6] D. Bicho, S. Pina, R.L. Reis, J.M. Oliveira, Commercial Products for Osteochondral Tissue Repair and Regeneration, Osteochondral Tissue Engineering, Springer2018, pp. 415-428.

[7] I. Martin, S. Miot, A. Barbero, M. Jakob, D. Wendt, Osteochondral tissue engineering, J. Biomech. 40(4) (2007) 750-765.

[8] K.J. Jones, B.V. Kelley, A. Arshi, D.R. McAllister, P.D. Fabricant, Comparative effectiveness of cartilage repair with respect to the minimal clinically important difference, The American journal of sports medicine (2019) 0363546518824552.

[9] A.J. Sophia Fox, A. Bedi, S.A. Rodeo, The basic science of articular cartilage: structure, composition, and function, Sports health 1(6) (2009) 461-468.

[10] H. Liang, T. Ji, Y. Zhang, Y. Wang, W. Guo, Reconstruction with 3D-printed pelvic endoprostheses after resection of a pelvic tumour, Bone Joint J. 99(2) (2017) 267-275.

[11] W.M. Groen, P. Diloksumpan, P.R. van Weeren, R. Levato, J. Malda, From intricate to integrated: Biofabrication of articulating joints, Journal of Orthopaedic Research 35(10) (2017) 2089-2097.

[12] R.F. LaPrade, L.S. Bursch, E.J. Olson, V. Havlas, C.S. Carlson, Histologic and immunohistochemical characteristics of failed articular cartilage resurfacing procedures for osteochondritis of the knee: a case series, The American journal of sports medicine 36(2) (2008) 360-368.

[13] I. Khan, S. Gilbert, S. Singhrao, V. Duance, C. Archer, Cartilage integration: evaluation of the reasons for failure of integration during cartilage repair. A review, Eur. Cell. Mater. 16(2008) (2008) 26-39.

[14] D.J. Huey, J.C. Hu, K.A. Athanasiou, Unlike bone, cartilage regeneration remains elusive, Science 338(6109) (2012) 917-921.

[15] S. Lopa, H. Madry, Bioinspired scaffolds for osteochondral regeneration, Tissue engineering Part A 20(15-16) (2014) 2052-2076.

[16] A. Di Luca, I. Lorenzo-Moldero, C. Mota, A. Lepedda, D. Auhl, C. Van Blitterswijk, L. Moroni, Tuning cell differentiation into a 3D scaffold presenting a pore shape gradient for osteochondral regeneration, Adv. Healthc. Mater. 5(14) (2016) 1753-1763.

[17] T. Xu, K.W. Binder, M.Z. Albanna, D. Dice, W. Zhao, J.J. Yoo, A. Atala, Hybrid printing of mechanically and biologically improved constructs for cartilage tissue engineering applications, Biofabrication 5(1) (2012) 015001.

[18] B. Balakrishnan, R. Banerjee, Biopolymer-based hydrogels for cartilage tissue engineering, Chem. Rev. 111(8) (2011) 4453-4474.

[19] C. Vinatier, D. Mrugala, C. Jorgensen, J. Guicheux, D. Noël, Cartilage engineering: a crucial combination of cells, biomaterials and biofactors, Trends Biotechnol. 27(5) (2009) 307-314.

[20] S. Stichler, T. Böck, N. Paxton, S. Bertlein, R. Levato, V. Schill, W. Smolan, J. Malda, J. Teßmar, T. Blunk, Double printing of hyaluronic acid/poly (glycidol) hybrid hydrogels with poly $(\varepsilon-$ caprolactone) for MSC chondrogenesis, Biofabrication 9(4) (2017) 044108. 
[21] T. Negoro, Y. Takagaki, H. Okura, A. Matsuyama, Trends in clinical trials for articular cartilage repair by cell therapy, Regen. Med. 3(1) (2018) 17.

[22] J. Visser, F.P. Melchels, J.E. Jeon, E.M. Van Bussel, L.S. Kimpton, H.M. Byrne, W.J. Dhert, P.D. Dalton, D.W. Hutmacher, J. Malda, Reinforcement of hydrogels using three-dimensionally printed microfibres, Nature communications 6 (2015) 6933.

[23] R. Levato, W.R. Webb, I.A. Otto, A. Mensinga, Y. Zhang, M. van Rijen, R. van Weeren, I.M. Khan, J. Malda, The bio in the ink: cartilage regeneration with bioprintable hydrogels and articular cartilage-derived progenitor cells, Acta biomaterialia 61 (2017) 41-53.

[24] V.H. Mouser, R. Levato, L.J. Bonassar, D.D. D'Lima, D.A. Grande, T.J. Klein, D.B. Saris, M. Zenobi-Wong, D. Gawlitta, J. Malda, Three-dimensional bioprinting and its potential in the field of articular cartilage regeneration, Cartilage 8(4) (2017) 327-340.

[25] M. Cucchiarini, H. Madry, F. Guilak, D. Saris, M. Stoddart, M. Koon Wong, P. Roughley, A vision on the future of articular cartilage repair, Eur. Cell. Mater. 27(12) (2014) 6.

[26] C.J. Moran, A. Ramesh, P.A. Brama, J.M. O'Byrne, F.J. O'Brien, T.J. Levingstone, The benefits and limitations of animal models for translational research in cartilage repair, Journal of experimental orthopaedics 3(1) (2016) 1.

[27] J. Malda, K. Benders, T. Klein, J. De Grauw, M. Kik, D. Hutmacher, D. Saris, P. Van Weeren, W. Dhert, Comparative study of depth-dependent characteristics of equine and human osteochondral tissue from the medial and lateral femoral condyles, Osteoarthritis Cartilage 20(10) (2012) 1147-1151.

[28] A.J. Nixon, L. Begum, H.O. Mohammed, B. Huibregtse, M.M. O'callaghan, G.L. Matthews, Autologous chondrocyte implantation drives early chondrogenesis and organized repair in extensive full-and partial-thickness cartilage defects in an equine model, J. Orthop. Res. 29(7) (2011) 1121-1130.

[29] D. Frisbie, S. Bowman, H. Colhoun, E. DiCarlo, C. Kawcak, C. Mcllwraith, Evaluation of autologous chondrocyte transplantation via a collagen membrane in equine articular defectsresults at 12 and 18 months, Osteoarthritis Cartilage 16(6) (2008) 667-679.

[30] M.M. Wilke, D.V. Nydam, A.J. Nixon, Enhanced early chondrogenesis in articular defects following arthroscopic mesenchymal stem cell implantation in an equine model, J. Orthop. Res. 25(7) (2007) 913-925.

[31] K. Pelttari, A. Winter, E. Steck, K. Goetzke, T. Hennig, B.G. Ochs, T. Aigner, W. Richter, Premature induction of hypertrophy during in vitro chondrogenesis of human mesenchymal stem cells correlates with calcification and vascular invasion after ectopic transplantation in SCID mice, Arthritis \& Rheumatism: Official Journal of the American College of Rheumatology 54(10) (2006) 3254-3266.

[32] G.P. Dowthwaite, J.C. Bishop, S.N. Redman, I.M. Khan, P. Rooney, D.J. Evans, L. Haughton, Z. Bayram, S. Boyer, B. Thomson, The surface of articular cartilage contains a progenitor cell population, Journal of cell science 117(6) (2004) 889-897.

[33] R. Williams, I.M. Khan, K. Richardson, L. Nelson, H.E. McCarthy, T. Analbelsi, S.K. Singhrao, G.P. Dowthwaite, R.E. Jones, D.M. Baird, Identification and clonal characterisation of a progenitor cell sub-population in normal human articular cartilage, PLoS ONE 5(10) (2010) e13246.

[34] D.D. Frisbie, H.E. McCarthy, C.W. Archer, M.F. Barrett, C.W. Mcllwraith, Evaluation of articular cartilage progenitor cells for the repair of articular defects in an equine model, JBJS 97(6) (2015) 484-493.

[35] H.E. McCarthy, J.J. Bara, K. Brakspear, S.K. Singhrao, C.W. Archer, The comparison of equine articular cartilage progenitor cells and bone marrow-derived stromal cells as potential cell sources for cartilage repair in the horse, Vet. J. 192(3) (2012) 345-351.

[36] B.L. Schumacher, J.A. Block, T.M. Schmid, M.B. Aydelotte, K.E. Kuettner, A novel proteoglycan synthesized and secreted by chondrocytes of the superficial zone of articular cartilage, Arch. Biochem. Biophys. 311(1) (1994) 144-152.

[37] V.H.M. Mouser, R. Levato, A. Mensinga, W.J.A. Dhert, D. Gawlitta, J. Malda, Bio-ink development for three-dimensional bioprinting of hetero-cellular cartilage constructs, Connect. Tissue Res. (2018) 1-15.

[38] V.H. Mouser, N.M. Dautzenberg, R. Levato, M.H. van Rijen, W.J. Dhert, J. Malda, D. Gawlitta, 
Ex vivo model unravelling cell distribution effect in hydrogels for cartilage repair, Altex 35(1) (2018) 65-76.

[39] I.A. Mancini, R.A. Vindas Bolaños, H. Brommer, M. Castilho, A. Ribeiro, J.P. Van Loon, A. Mensinga, M.H. Van Rijen, J. Malda, R. van Weeren, Fixation of hydrogel constructs for cartilage repair in the equine model: a challenging issue, Tissue Engineering Part C: Methods 23(11) (2017) 804-814.

[40] T. Bock, V. Schill, M. Krahnke, A.F. Steinert, J. Tessmar, T. Blunk, J. Groll, TGF-beta1-Modified Hyaluronic Acid/Poly(glycidol) Hydrogels for Chondrogenic Differentiation of Human Mesenchymal Stromal Cells, Macromolecular bioscience 18(7) (2018) e1700390.

[41] Y.J. Kim, R.L. Sah, J.Y. Doong, A.J. Grodzinsky, Fluorometric assay of DNA in cartilage explants using Hoechst 33258, Analytical biochemistry 174(1) (1988) 168-76.

[42] R.W. Farndale, D.J. Buttle, A.J. Barrett, Improved quantitation and discrimination of sulphated glycosaminoglycans by use of dimethylmethylene blue, Biochimica et biophysica acta 883(2) (1986) 173-7.

[43] J.F. Woessner, Jr., The determination of hydroxyproline in tissue and protein samples containing small proportions of this imino acid, Archives of biochemistry and biophysics 93 (1961) 440-7.

[44] A.P. Hollander, T.F. Heathfield, C. Webber, Y. Iwata, R. Bourne, C. Rorabeck, A.R. Poole, Increased damage to type II collagen in osteoarthritic articular cartilage detected by a new immunoassay, J Clin Invest 93(4) (1994) 1722-32.

[45] I. Martin, B. Obradovic, L.E. Freed, G. Vunjak-Novakovic, Method for quantitative analysis of glycosaminoglycan distribution in cultured natural and engineered cartilage, Ann. Biomed. Eng. 27(5) (1999) 656-662.

[46] S. Bosch, F. Serra Bragança, M. Marin-Perianu, R. Marin-Perianu, B.J. van der Zwaag, J. Voskamp, W. Back, R. Van Weeren, P. Havinga, EquiMoves: a wireless networked inertial measurement system for objective examination of horse gait, Sensors 18(3) (2018) 850.

[47] M. Van Den Borne, N. Raijmakers, J. Vanlauwe, J. Victor, S. De Jong, J. Bellemans, D. Saris, International Cartilage Repair Society (ICRS) and Oswestry macroscopic cartilage evaluation scores validated for use in Autologous Chondrocyte Implantation (ACI) and microfracture, Osteoarthritis Cartilage 15(12) (2007) 1397-1402.

[48] P.R. Moshtagh, B. Pouran, N.M. Korthagen, A.A. Zadpoor, H. Weinans, Guidelines for an optimized indentation protocol for measurement of cartilage stiffness: The effects of spatial variation and indentation parameters, J. Biomech. 49(14) (2016) 3602-3607.

[49] L. Van de Goor, W. Van Haeringen, J. Lenstra, Population studies of 17 equine STR for forensic and phylogenetic analysis, Anim. Genet. 42(6) (2011) 627-633.

[50] P. Mainil-Varlet, B. Van Damme, D. Nesic, G. Knutsen, R. Kandel, S. Roberts, A new histology scoring system for the assessment of the quality of human cartilage repair: ICRS II, The American journal of sports medicine 38(5) (2010) 880-890.

[51] C. RDevelopment, TEAM 2009: R: A language and environment for statistical computing, Vienna, Austria. Internet: http://www. R-project. org (2012).

[52] R.V. Bolaños, S. Cokelaere, J.E. McDermott, K. Benders, U. Gbureck, S. Plomp, H. Weinans, J. Groll, P. van Weeren, J. Malda, The use of a cartilage decellularized matrix scaffold for the repair of osteochondral defects: the importance of long-term studies in a large animal model, Osteoarthritis and Cartilage 25(3) (2017) 413-420.

[53] P. Brun, S.C. Dickinson, B. Zavan, R. Cortivo, A.P. Hollander, G. Abatangelo, Characteristics of repair tissue in second-look and third-look biopsies from patients treated with engineered cartilage: relationship to symptomatology and time after implantation, Arthritis Res. Ther. 10(6) (2008) R132.

[54] M. de Ruijter, A. Ribeiro, I. Dokter, M. Castilho, J. Malda, Simultaneous micropatterning of fibrous meshes and bioinks for the fabrication of living tissue constructs, Adv. Healthc. Mater. (2018) 1800418.

[55] K.L. Caldwell, J. Wang, Cell-based articular cartilage repair: the link between development and regeneration, Osteoarthritis Cartilage 23(3) (2015) 351-62.

[56] M. Schnabel, S. Marlovits, G. Eckhoff, I. Fichtel, L. Gotzen, V. Vecsei, J. Schlegel, 
Dedifferentiation-associated changes in morphology and gene expression in primary human articular chondrocytes in cell culture, Osteoarthritis Cartilage 10(1) (2002) 62-70.

[57] G.-I. Im, Tissue engineering in osteoarthritis: current status and prospect of mesenchymal stem cell therapy, BioDrugs 32 (2018) 183-192.

[58] R.A. Somoza, J.F. Welter, D. Correa, A.I. Caplan, Chondrogenic differentiation of mesenchymal stem cells: challenges and unfulfilled expectations, Tissue Eng Part B Rev 20(6) (2014) 596-608.

[59] T.S. de Windt, L.A. Vonk, I. Slaper-Cortenbach, M.P. van den Broek, R. Nizak, M.H. van Rijen, R.A. de Weger, W.J. Dhert, D.B. Saris, Allogeneic mesenchymal stem cells stimulate cartilage regeneration and are safe for single-stage cartilage repair in humans upon mixture with recycled autologous chondrons, Stem Cells 35(1) (2017) 256-264.

[60] C.-C. Wei, A.B. Lin, S.-C. Hung, Mesenchymal stem cells in regenerative medicine for musculoskeletal diseases: bench, bedside, and industry, Cell Transplant. 23(4-5) (2014) 505-512.

[61] R.L. Sah, A. Ratcliffe, Translational models for musculoskeletal tissue engineering and regenerative medicine, Tissue Eng. Part B Rev. 16(1) (2010) 1-3.

[62] M.B. Hurtig, M.D. Buschmann, L.A. Fortier, C.D. Hoemann, E.B. Hunziker, J.S. Jurvelin, P. Mainil-Varlet, C.W. Mcllwraith, R.L. Sah, R.A. Whiteside, Preclinical studies for cartilage repair: recommendations from the International Cartilage Repair Society, Cartilage 2(2) (2011) 137-152.

[63] A. Chevrier, A.S. Kouao, G. Picard, M.B. Hurtig, M.D. Buschmann, Interspecies comparison of subchondral bone properties important for cartilage repair, J. Orthop. Res. 33(1) (2015) 63-70.

[64] M.W. Pot, V.K. Gonzales, P. Buma, J. IntHout, T.H. van Kuppevelt, R.B. de Vries, W.F. Daamen, Improved cartilage regeneration by implantation of acellular biomaterials after bone marrow stimulation: a systematic review and meta-analysis of animal studies, PeerJ 4 (2016) e2243.

[65] E. Kon, A. Roffi, G. Filardo, G. Tesei, M. Marcacci, Scaffold-based cartilage treatments: with or without cells? A systematic review of preclinical and clinical evidence, Arthroscopy: The Journal of Arthroscopic \& Related Surgery 31(4) (2015) 767-775.

[66] E. Salonius, L. Rieppo, M.J. Nissi, H.J. Pulkkinen, H. Brommer, A. Brünott, T.S. Silvast, P.R. Van Weeren, V. Muhonen, P.A. Brama, Critical-sized cartilage defects in the equine carpus, Connective Tissue Research 60(2) (2019) 95-106.

[67] J.K. Sarin, N.C. Te Moller, I.A. Mancini, H. Brommer, J. Visser, J. Malda, P.R. van Weeren, I.O. Afara, J. Töyräs, Arthroscopic near infrared spectroscopy enables simultaneous quantitative evaluation of articular cartilage and subchondral bone in vivo, Scientific reports 8(1) (2018) 13409.

[68] A. Nixon, L. Fortier, L. Goodrich, N. Ducharme, Arthroscopic reattachment of osteochondritis dissecans lesions using resorbable polydioxanone pins, Equine Vet. J. 36(5) (2004) 376-383.

[69] J. Bekkers, A. Tsuchida, J. Malda, L. Creemers, R. Castelein, D.B. Saris, W. Dhert, Quality of scaffold fixation in a human cadaver knee model, Osteoarthritis and cartilage 18(2) (2010) 266272.

[70] J.-p. Seo, T. Tanabe, N. Tsuzuki, S. Haneda, K. Yamada, H. Furuoka, Y. Tabata, N. Sasaki, Effects of bilayer gelatin/ $\beta$-tricalcium phosphate sponges loaded with mesenchymal stem cells, chondrocytes, bone morphogenetic protein-2, and platelet rich plasma on osteochondral defects of the talus in horses, Res. Vet. Sci. 95(3) (2013) 1210-1216.

[71] N. Tsuzuki, K. Otsuka, J. Seo, K. Yamada, S. Haneda, H. Furuoka, Y. Tabata, N. Sasaki, In vivo osteoinductivity of gelatin $\beta$-tri-calcium phosphate sponge and bone morphogenetic protein-2 on an equine third metacarpal bone defect, Res. Vet. Sci. 93(2) (2012) 1021-1025.

[72] F. Bothe, A.-K. Deubel, E. Hesse, B. Lotz, J. Groll, C. Werner, W. Richter, S. Hagmann, Treatment of focal cartilage defects in minipigs with zonal chondrocyte/mesenchymal progenitor cell constructs, Int. J. Mol. Sci. 20(3) (2019) 653.

[73] D.W. Hutmacher, Scaffolds in tissue engineering bone and cartilage, The Biomaterials: Silver Jubilee Compendium, Elsevier2000, pp. 175-189.

[74] J.K. Mouw, J.T. Connelly, C.G. Wilson, K.E. Michael, M.E. Levenston, Dynamic compression regulates the expression and synthesis of chondrocyte-specific matrix molecules in bone marrow stromal cells, Stem Cells 25(3) (2007) 655-663. 


\section{SUPPLEMENTAL DATA}

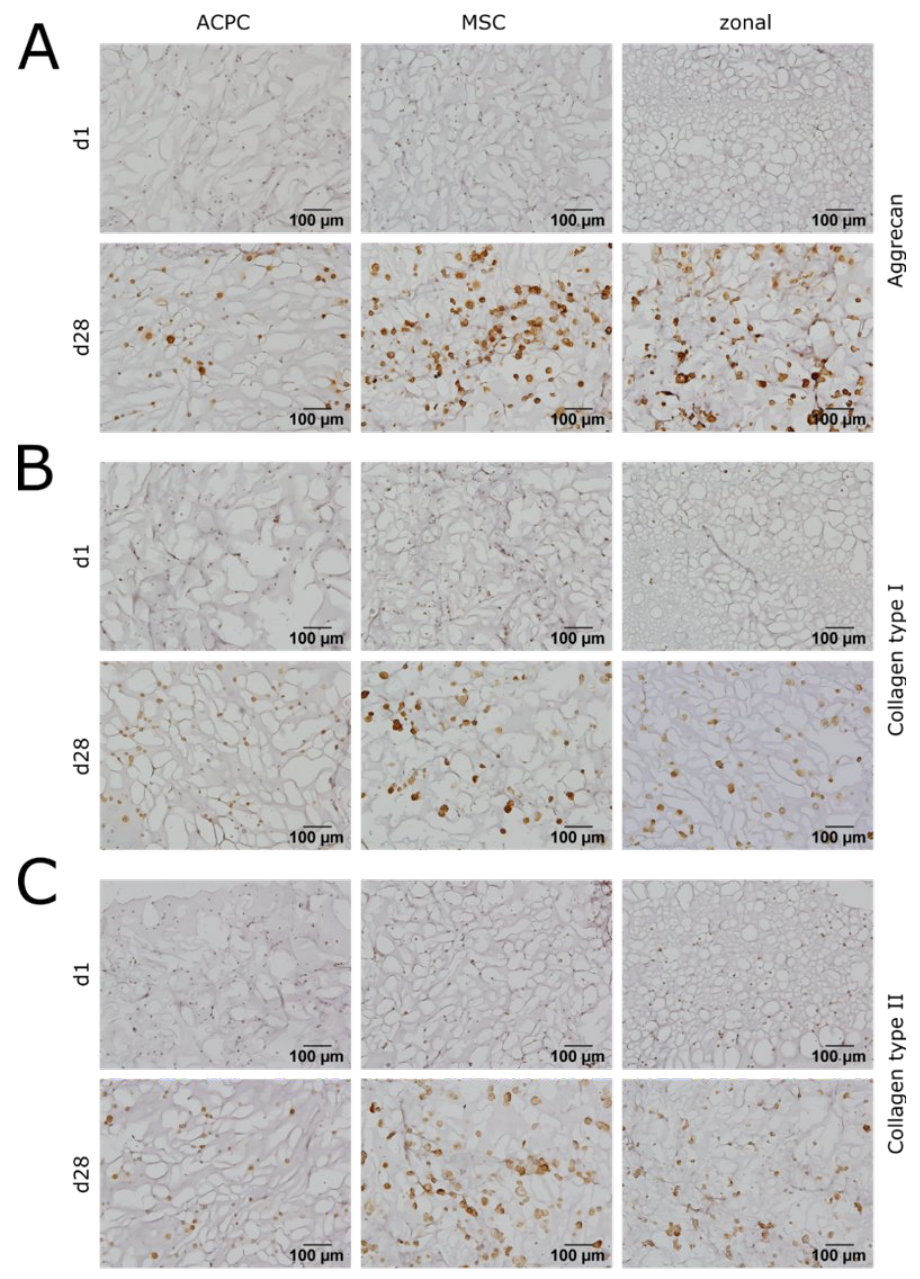

Figure S1. Immunohistochemical stainings of (HA-SH/P(AGE-CO-G)) hydrogel constructs, seeded with $20 \times 10^{6}$ cells $/ \mathrm{mL}$, after 1 and 28 d of chondrogenic differentiation. Immunohistochemical stainings for (A) Aggrecan, (B) collagen type I and (C) collagen type II. Mayer's hematoxylin was used as counterstain for cell nuclei. The holes in the hydrogel are artefacts derived from dehydration and rehydration of the hydrogel during sample preparation. Three independent experiments with $n=3$ biological replicates each were performed. Representative data from one experiment is shown. Scale bars represent $100 \mu \mathrm{m}$. 
A
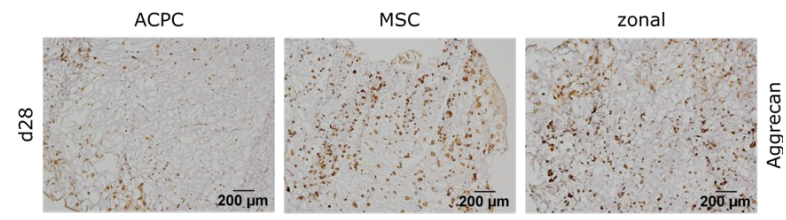

B
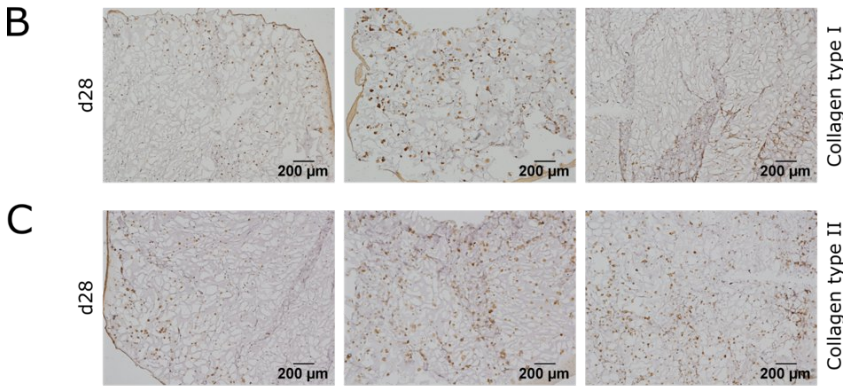

Figure S2. Immunohistochemical stainings of (HA-SH/P(AGE-CO-G)) hydrogel constructs, seeded with $20 \times 10^{6}$ cells $/ \mathrm{mL}$, after $28 \mathrm{~d}$ of chondrogenic differentiation for (A) aggrecan, (B) collagen type I and (C) collagen type II. Overviews are shown to illustrate distribution of extracellular matrix produced by ACPCS or MSCs within the hydrogel construct. Mayer's hematoxylin was used as counterstain for cell nuclei. The holes in the hydrogel are artefacts derived from dehydration and rehydration of the hydrogel during sample preparation. Three independent experiments with $n=3$ biological replicates each were performed. Representative data from one experiment is shown. Scale bars represent $200 \mu \mathrm{m}$.
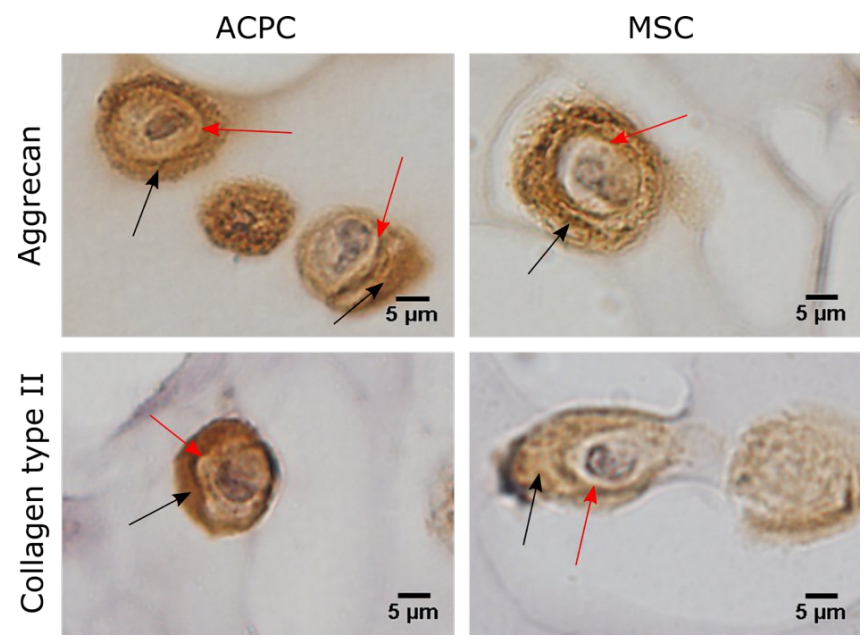

Figure S3. Immunohistochemical stainings of (HA-SH/P(AGE-CO-G)) hydrogel constructs, seeded with $20 \times 10^{6} \mathrm{cells} / \mathrm{mL}$ after $28 \mathrm{~d}$ of chondrogenic differentiation. High magnification images of ACPCS and MSCS are shown to demonstrate the intra- and mostly pericellular distribution of extracellular matrix components like glycosaminoglycans and collagen type II. Black arrows indicate pericellular matrix and red arrows indicate cell boundaries. Nuclei were stained using Mayer's hematoxylin. Three independent experiments with $n=3$ biological replicates each were performed. Representative data from one experiment is shown. Scale bars represent $5 \mu \mathrm{m}$. 


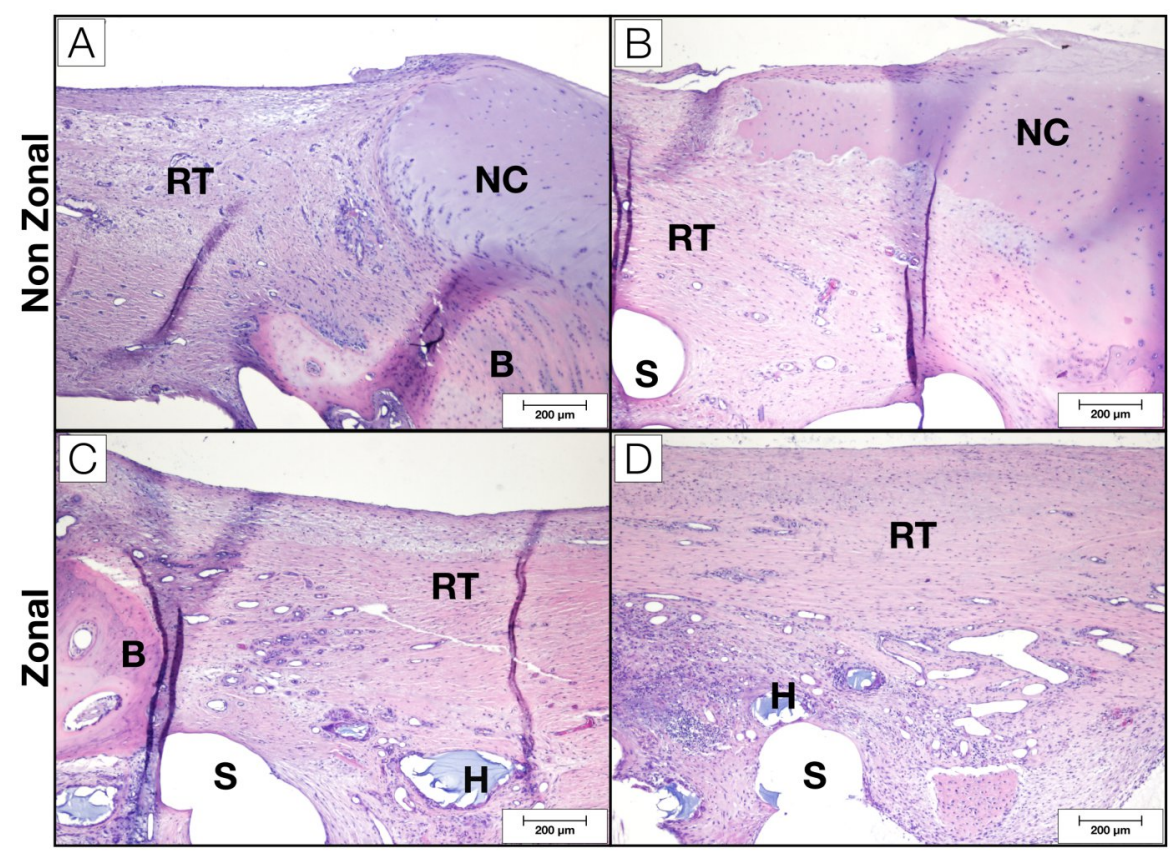

Figure S4. (A, B) Hematoxylin eosin staining of cartilage portion of the non-zonal defects. $(C, D)$ Hematoxylin eosin staining of cartilage portion of the zonal defects. Newly formed repair tissue appeared well integrated with adjacent native cartilage (NC), and underlying bone (B), completely filling the scaffold $(S$, empty space left after dissolution during histological processing). Within the newly formed tissue, fragments of hydrogel $(H)$ could still be observed. 

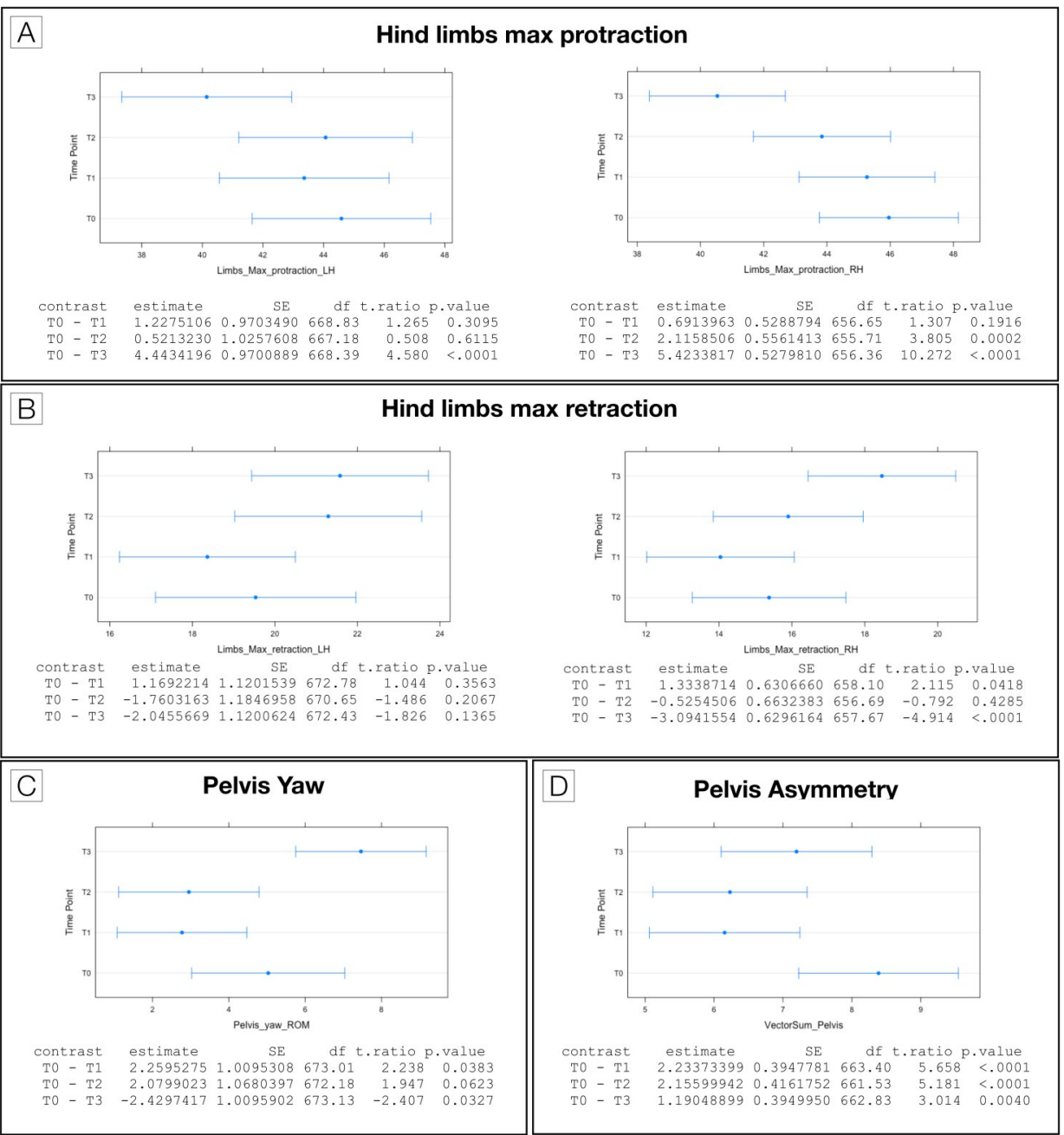

Figure S5. Summary of objective gait analysis changes over time (TO represents the baseline, T1, $T 2$ and T3 respectively 2, 4 and 6 months post-operatively), performed with the QHorse system as an outcome parameter for lameness. (A) For both hindlimbs, max protraction angle was reduced after surgery and remained reduced until the last time point (5 deg reduction, $p<0.001$ ). (B) Maximal retraction of the hindlimbs was initially reduced after surgery and increased over time, when compared to baseline (2.5 deg, $p<0.001)$. (C) Pelvis rotation along the horizontal plane (yaw) was reduced after surgery and increased at the last time point when compared to baseline (2.4 deg, $p=0.033)$. (D) Vertical motion symmetry of the pelvis in the sagittal plane reduced after surgery $(1.2 \mathrm{~mm}, p=0.004)$. 


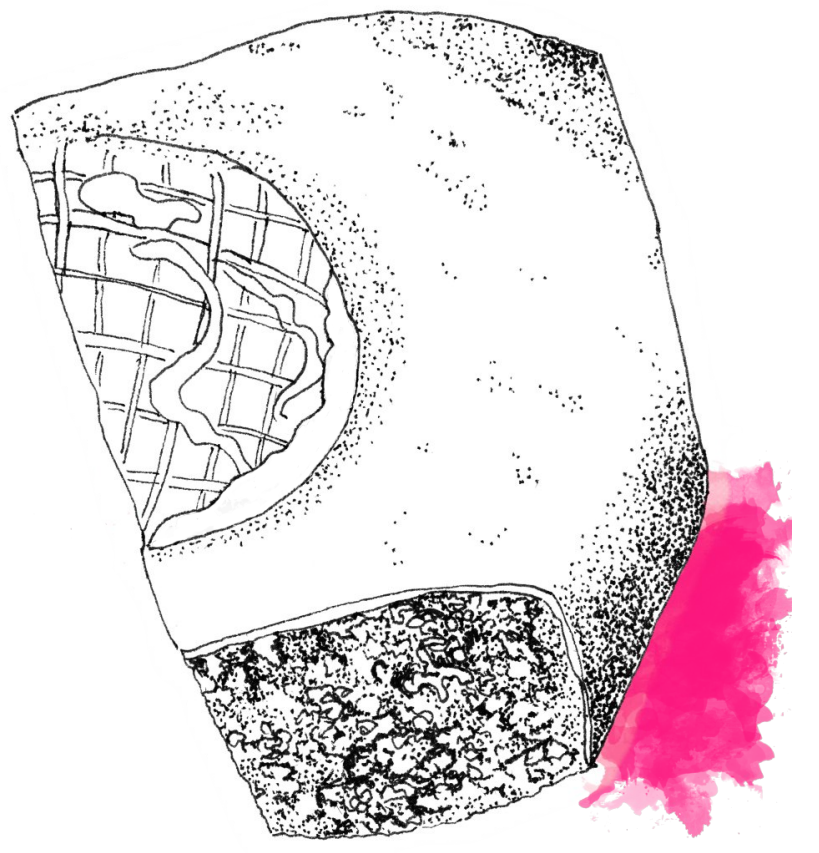




\title{
Chapter
}

Addendum to: "A composite hydrogel3D printed thermoplast osteochondral anchor as example for a zonal approach to cartilage repair: in vivo performance in a long-term equine model"

\author{
I.A.D. Mancini \\ J. Malda \\ R. Levato \\ H. Brommer \\ F. Schrön \\ C. Warner \\ P.R. van Weeren
}

Submitted 


\section{ADDENDUM}

In this addendum, we would like to comment on and add to the abovementioned study that was performed by our group and recently published in Biofabrication [1]. This study focused on the possible effect of a zonal configuration of the cellular component within an osteochondral implant consisting of a 3D-printed poly(e-caprolactone) (PCL) bone anchor with chondral reinforcing fibers combined with a hydrogel during a 6 month in vivo study in 8 healthy Shetland ponies. Using allogeneic cells, in the top layer of the hydrogel Articular Cartilage Progenitor Cells (ACPCS) were encapsulated, while the bottom layer contained bone marrow-derived Mesenchymal Stromal Cells (MSCs). This configuration was compared with an identical scaffold featuring a homogeneous distribution of MSCs. While the overall results with respect to cartilage regeneration were disappointing with formation of a mixture of fibrocartilage and more fibrous repair tissue. This was conjectured to be due to the combination of multiple factors, including the early loss of seeded cells and early degradation of the material. However, the interesting observation was made that the repair tissue of the zonal implants was significantly stiffer than that of the non-zonal implants, although still substantially less than that of native cartilage.

The published study was part of a twin study, during which another hydrogel was also tested in an identical setting. Given the large similarities in experimental set-up and also in outcome of both studies, we felt that publication of the second half as a full paper was not warranted. Moreover, the very different nature of the hydrogel system used in the second study did not fit entirely to justify merging of the two studies. However the additional conclusions, which can be drawn when considering both studies together, make the information from the second study of interest to the readership of this journal.

The hydrogel described in the publication was a thiol-ene cross-linkable hyaluronic acid/poly(glycidol) hybrid hydrogel (HA-SH / P(AGE-co-G)), which had been chosen based on the previous promising in vitro results regarding chondrogenesis and simultaneous tailoring of chemical and physical properties $[2,3]$. In the second study, a conceptually different poly-ethylene glycol PEGbased hydrogel, which also had been shown to support chondrogenesis in 
vitro, was used [4]. The modifiable composition and degradation rate was also deemed as an advantage of this particular class of hydrogels [5]. Earlier research showed that modifications of PEG-hydrogels with cell-instructive signals had allowed to modulate tissue regeneration [6]: addition of matrix metalloproteinase (MMP) degradable linkers resulted in the increased production of GAGs [4], while heparin allowed independent tuning of the mechanical and biomolecular properties of the hydrogel [7, 8]. This combination of modifications yielded positive results when evaluated in in vivo ectopic models. A first study by Hesse et al., showed the possibility of using a functionalized starPEG/heparin hydrogel to direct cartilage matrix distribution, and investigated different concentrations of MMP-linkers to modify the biomaterial's degradation rate [9]. The possibility to manipulate this degradation rate of the hydrogel is a very interesting feature of materials that are intended for use in cartilage repair, as the tissue is heavily loaded and longterm retainment of a reasonable degree of stiffness is needed [10,11]. In this context, the rapid degradation and associated loss of biomechanical characteristics of hydrogels used for cartilage repair has been signaled as a potential problem in various publications $[12,13]$. Furthermore, a study by Kunisch et al. demonstrated the ability of a starPEG/heparin hydrogel construct laden with MSCs and chondrocytes in a murine model to successfully allow growth of a stable calcified cartilage layer [14]. These studies represented hence a promising base to address the repair of full osteochondral defects by combining our layered design with a tailored composition of starPEG/heparin. Therefore, a long-term evaluation in a previously established large animal model [11] seemed warranted.

The experimental design was equal to the one reported earlier [1] and a composite construct, with a 3D-printed $\mathrm{PCL}$ bone anchor with chondral reinforcement and two layers of starPEG/heparin (20\% MMP degradable, 0.5 $\mathrm{mol} \mathrm{RGD} / \mathrm{mol}$ heparin), was fabricated. As in the previous study, the top layer contained ACPCs, and the bottom layer contained MSCs. Seven Shetland ponies were used and in vivo monitoring was identical. Examinations included quantitative gait analysis with a wireless networked inertial measurement system [15] before and 2, 4 and 6 months after surgery, as well as second look arthroscopies at 4 and 6 months after surgery. Upon completion of the study, assessment of repair tissue was executed with biomechanical characterization, 


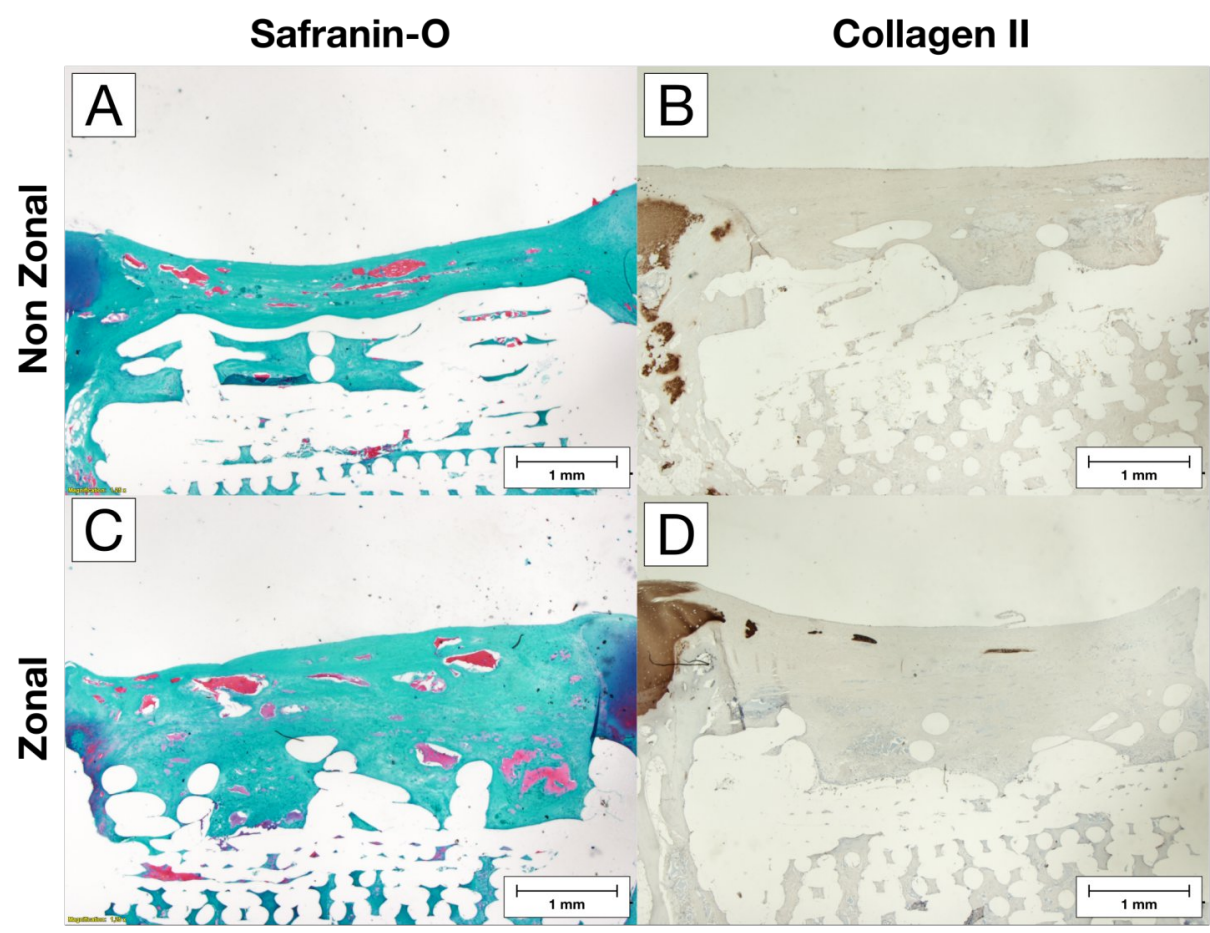

Figure 1. Histological sections of osteochondral defects repaired with zonal and non-zonal constructs. All defects were completely filled with repair tissue, which appeared well integrated with the adjacent native tissue. $(A, C)$ Sections were stained with safranin- $O$, showing remnants of the starPEG hydrogel (red), integration with the surrounding native tissue, and limited GAG loss of the cartilage adjacent the defect. $(B, D)$ Histological sections stained with collagen type II showed virtually no collagen within the repair tissue filling the defects.

histological analysis and micro-CT evaluation.

Despite the very different hydrogel system that had been used, the results in terms of tissue repair of the second study were to a very large extent similar to those observed in the first study. Clinically, the animals performed well with virtually no alterations in gait pattern and relook arthroscopy at 4 months showed a similar defect filling and integration with the surrounding native tissue. After 6 months in vivo, microCT also revealed bone ingrowth into the anchor of the implant, similar to the observations in the first study. Histologically, a mixture of fibrous and cartilage-like repair tissue filled the defects (Fig. 1), equally in zonal and non-zonal constructs, and scoring did not highlight any significant differences. Degradation of the starPEG hydrogel was slower as exemplified by the visible remnants of the hydrogel (Fig. 1A, C), 


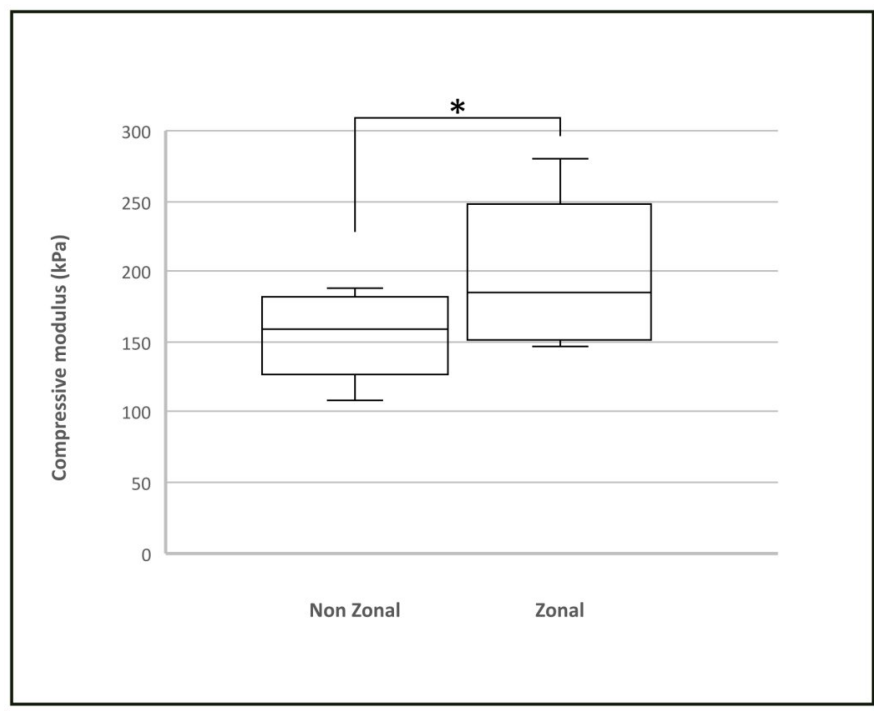

Figure 2. Biomechanical characterization of newly formed tissue filling the defects with microindentation. Average value of the compressive modulus in the zonal group was $197.3 \pm 49.3 \mathrm{kPa}$, while in the defects repaired with non-zonal constructs, the average compressive modulus was significantly lower (153.2 $\pm 30.0 \mathrm{kPa}, \mathrm{p}<0.05)$. A minimum of 3 measurements were performed in different areas of the defect.

which had not been the case for the HA-based gel. Interestingly, the repair tissue was again significantly stiffer in defects repaired with zonal constructs (Fig. 2). In absolute terms, mean stiffness was higher in the zonal and nonzonal variants of the starPEG group (197 and $153 \mathrm{KPa}$, respectively) than in the HA group (148 and $97 \mathrm{KPa}$, respectively), though both were still considerably less than the stiffness of healthy tissue of the same animals (approximately $500 \mathrm{Kpa})$.

It is interesting to note that this second investigation yielded results that were very similar to the original study, despite the very different biomaterial used as hydrogel. The expected slower degradation rate indeed resulted in longer presence of remnants of the original hydrogel. However, this did not affect overall quality of the repair tissue, which still mainly consisted of fibrocartilage with virtually no collagen type II. The overall compressive stiffness was slightly higher in the repair tissue formed in the defects treated with the starPEG-containing implants than in those treated with the HA-based implants, irrespective of the presence of two or only a single cell type. This may be, at least in part, related to the increased presence of the hydrogel, as characterization with micro-indentation of in vitro cultured constructs showed 
compressive values of similar orders of magnitude [16]. However, it cannot be determined how these values -obtained in a controlled in vitro situation-, would relate to constructs that have undergone fragmentation and 6 months long in vivo implantation.

The zonal configuration appeared to have no major effects in both studies, but it produced a repair tissue with higher compressive modulus in both cases. The consistency in the findings over the two studies is interesting and lends credibility to these observations, however, no conclusive explanation can be given. Most likely, the combination of the two cell types has led to a (slight) difference in composition or configuration of the extracellular matrix (ECM) that was produced. It is clear that collagen type II is not the determining factor here, as production was minimal in both studies. The analytical methods that were used for post-mortem analysis, however, did not permit conclusions on either molecular peculiarities of the proteoglycan components of the ECM or details of its architecture.

It can be concluded from the two studies that the bone compartment behaved satisfactorily, but that the cartilage phase did not result in the generation of acceptable repair tissue. The sub-optimal characteristics of the repair tissue may be due to a multitude of factors, but the type of hydrogel used did not seem to have a major influence. Initial lack and early loss of biomechanical resistance may be an important factor here, as recently highlighted [17]. Therefore, alternative approaches like in vitro pre-culture of the implants or their reinforcement with materials that retain strength for much longer, should be actively explored. 


\section{REFERENCES}

[1] I.A. Mancini, S. Schmidt, H. Brommer, B. Pouran, S. Schäfer, J. Tessmar, A. Mensinga, M.H. van Rijen, J. Groll, T. Blunk, A composite hydrogel-3D printed thermoplast osteochondral anchor as an example for a zonal approach to cartilage repair: in vivo performance in a long-term equine model, Biofabrication (2020).

[2] S. Stichler, T. Böck, N. Paxton, S. Bertlein, R. Levato, V. Schill, W. Smolan, J. Malda, J. Teßmar, T. Blunk, Double printing of hyaluronic acid/poly (glycidol) hybrid hydrogels with poly $(\varepsilon-$ caprolactone) for MSC chondrogenesis, Biofabrication 9(4) (2017) 044108.

[3] S. Stichler, T. Jungst, M. Schamel, I. Zilkowski, M. Kuhlmann, T. Böck, T. Blunk, J. Teßmar, J. Groll, Thiol-ene clickable poly (glycidol) hydrogels for biofabrication, Annals of biomedical engineering 45(1) (2017) 273-285.

[4] S.B. Anderson, C.-C. Lin, D.V. Kuntzler, K.S. Anseth, The performance of human mesenchymal stem cells encapsulated in cell-degradable polymer-peptide hydrogels, Biomaterials 32(14) (2011) 3564-3574.

[5] P.B. Welzel, S. Prokoph, A. Zieris, M. Grimmer, S. Zschoche, U. Freudenberg, C. Werner, Modulating biofunctional starPEG heparin hydrogels by varying size and ratio of the constituents, Polymers 3(1) (2011) 602-620.

[6] J.J. Rice, M.M. Martino, L. De Laporte, F. Tortelli, P.S. Briquez, J.A. Hubbell, Engineering the regenerative microenvironment with biomaterials, Advanced healthcare materials 2(1) (2013) 57-71.

[7] M.V. Tsurkan, K. Chwalek, S. Prokoph, A. Zieris, K.R. Levental, U. Freudenberg, C. Werner, Defined polymer-peptide conjugates to form cell-instructive starPEG-heparin matrices in situ, Advanced Materials 25(18) (2013) 2606-2610.

[8] S.E. Sakiyama-Elbert, Incorporation of heparin into biomaterials, Acta biomaterialia 10(4) (2014) 1581-1587.

[9] E. Hesse, U. Freudenberg, T. Niemietz, C. Greth, M. Weisser, S. Hagmann, M. Binner, C. Werner, W. Richter, Peptide-functionalized starPEG/heparin hydrogels direct mitogenicity, cell morphology and cartilage matrix distribution in vitro and in vivo, Journal of tissue engineering and regenerative medicine 12(1) (2018) 229-239.

[10] A. Armiento, M. Stoddart, M. Alini, D. Eglin, Biomaterials for articular cartilage tissue engineering: Learning from biology, Acta biomaterialia 65 (2018) 1-20.

[11] I.A. Mancini, R.A. Vindas Bolaños, H. Brommer, M. Castilho, A. Ribeiro, J.P. Van Loon, A. Mensinga, M.H. Van Rijen, J. Malda, R. van Weeren, Fixation of hydrogel constructs for cartilage repair in the equine model: a challenging issue, Tissue Engineering Part C: Methods 23(11) (2017) 804-814.

[12] D.J. Huey, J.C. Hu, K.A. Athanasiou, Unlike bone, cartilage regeneration remains elusive, Science 338(6109) (2012) 917-921.

[13] D.D. Frisbie, H.E. McCarthy, C.W. Archer, M.F. Barrett, C.W. Mcllwraith, Evaluation of articular cartilage progenitor cells for the repair of articular defects in an equine model, JBJS 97(6) (2015) 484-493.

[14] E. Kunisch, A.-K. Knauf, E. Hesse, U. Freudenberg, C. Werner, F. Bothe, S. Diederichs, W. Richter, StarPEG/heparin-hydrogel based in vivo engineering of stable bizonal cartilage with a calcified bottom layer, Biofabrication 11(1) (2018) 015001.

[15] S. Bosch, F. Serra Bragança, M. Marin-Perianu, R. Marin-Perianu, B.J. Van der Zwaag, J. Voskamp, W. Back, R. Van Weeren, P. Havinga, Equimoves: A wireless networked inertial measurement system for objective examination of horse gait, Sensors 18(3) (2018) 850.

[16] F. Schrön, Inkjet bioprinting and 3D culture of human MSC-laden binary starPEG-heparin hydrogels for cartilage tissue engineering.

[17] J. Malda, J. Groll, P.R. van Weeren, Rethinking articular cartilage regeneration based on a 250-year-old statement, Nature Reviews Rheumatology (2019) 1. 


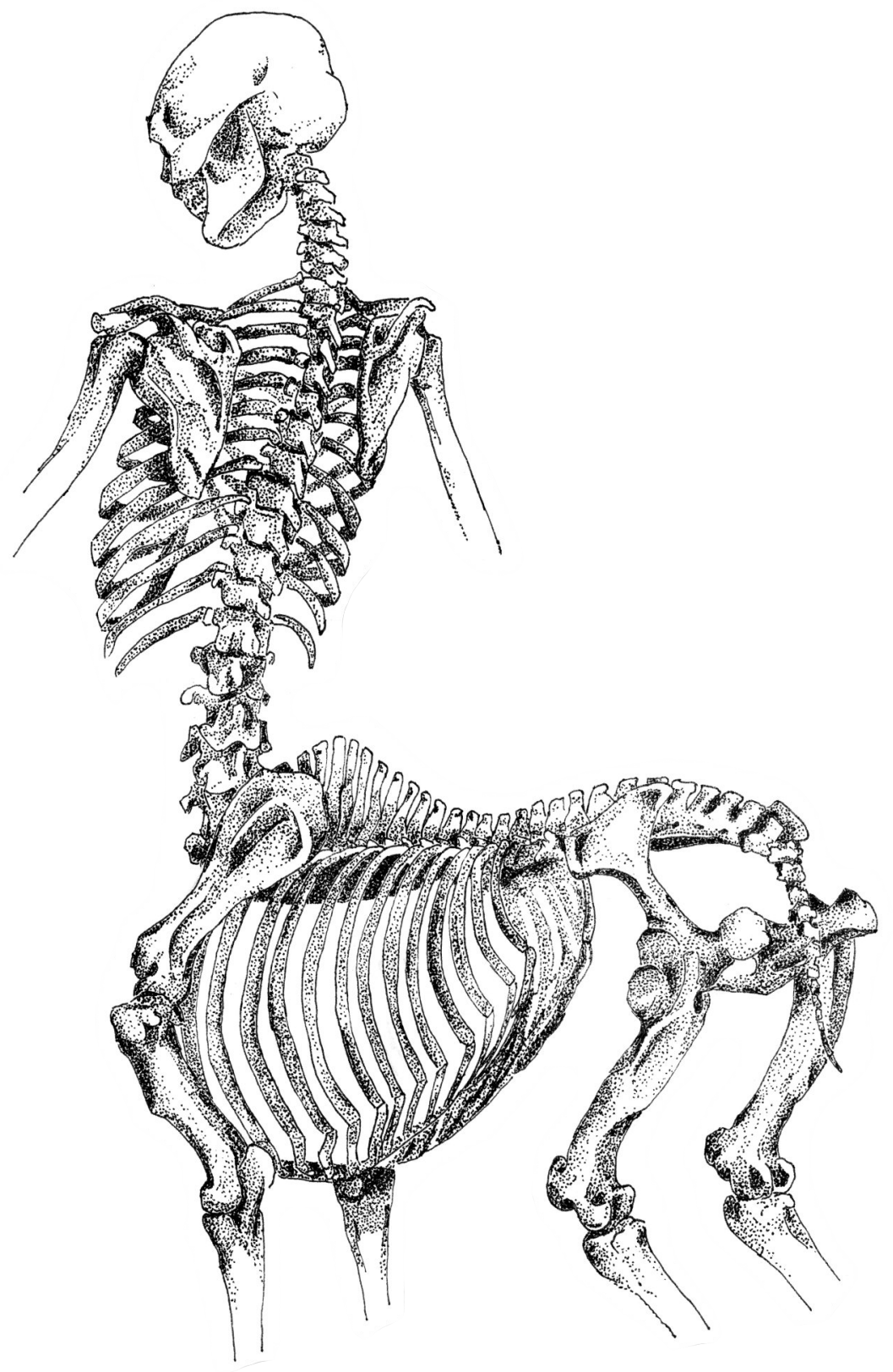




\section{Chapter}

General discussion 


\section{DISCUSSION}

Over the last decades, life expectancy has increased dramatically around the globe. According to United Nations records, a person born in 1960, could expect to live to 52 years of age, while today the average is 72 years, and over 80 years in developed areas like Europe [1]. The rapid advancement of modern medicine and public health policies in the last few decades have made it possible for most humans to live substantially longer than many of our -even not too distant- forebears. However, this trend seems to be plateauing, as the increase in life expectancy has slowed down sensibly over the last years [2], suggesting that medicine may have reached its limits in pushing further the extension of human life. Concurrently, modern day healthcare is posed with the challenge of addressing the increasing prevalence in chronic and degenerative diseases in the aging population. This could potentially be realized with therapies capable of limiting the degeneration of organs and tissues. A first solution to organ and tissue degeneration, was proposed in the 1950s, with the development of transplantation medicine, which allowed substitution of organs and tissues that were no longer functional, with healthy replacement organs from donors. This solution, however, came with significant limitations in terms of tissue availability and compatibility, as well as a life-long dependency on immune-suppressants with the associated risks and complications [3].

The emergence of the field of regenerative medicine (RM) brought on the application of stem cell research combined with biomedical engineering principles, opening the path for the regeneration of tissues and potentially entire organs ex novo [4]. This branch of medicine brought a paradigm shift to healthcare therapies, by focusing on facilitating and stimulating the body's own regenerative capacity. Since then, regenerative medicine showed promising potential for the treatment of many disorders, including musculoskeletal diseases for which there is a major and increasing clinical need. In fact, joint disease is a wide-spread phenomenon [5] with osteoarthritis (OA) being a leading cause of disability and affecting more than 90 million people in the US alone [6]. Clinically successful therapies have been developed, of which arthroplasty is the best example. However, these are very invasive, non-regenerative and costly procedures that last for a limited amount of time and alternative solutions are urgently needed. In the quest for repair of 
joint damage, restoration of cartilage function in particular, has proven to be a challenge that has not yet been overcome [7].

The conceptual basis of this thesis was that, before addressing approaches to restore cartilage and select models for the evaluation of novel therapies, it would be valuable to carefully consider Nature's template of the osteochondral unit. Herein, we aimed to achieve a better understanding of the essential compositional and architectural features that are required for proper functioning of the osteochondral unit (Chapters 2 and 3), and use these lessons in the translation of viable in vitro strategies to the preclinical equine animal model. The osteochondral unit of over 40 mammalian species was investigated with particular attention to how increasing body mass and different loading conditions affect the components and architecture of the unit. Through three long-term studies using the biomechanically challenging equine model, the hurdles of a cell-driven cartilage regeneration journey are shown and addressed. First, a single-step, direct casting surgical technique was used to assess the performance of a microfiber-reinforced, cell-laden hydrogel in chondral defects (Chapter 4). Next, the optimization of strategies to enhance fixation and tissue integration led to the development of a press-fit, multiphasic construct for the reconstruction of osteochondral defects (Chapter $5)$. This type of implant was used to evaluate the impact of zonal distribution of cells on tissue regeneration (Chapters 6 and 7).

\section{Part I}

How cartilage and bone tissue enable functionality: Nature's template

The musculoskeletal system has evolved to enable locomotion, and thereby to sustain and transmit the mechanical loads experienced both at rest and during motion. In particular, articulating joints have evolved to accommodate considerable loads and at the same time enable a near-frictionless relative displacement of bony segments. Hereto, joints have developed highly specialized tissues, whose architectures are attuned to cope with such loads [8]. The nature and magnitude of the forces exerted on joints vary tremendously, as can be calculated from body mass and the externally acting forces, such as gravity, buoyancy or drag, depending on the environment in 
which locomotion takes place.

Life in extremely different environments, such as in water or on land, and weights that range from a few grams (e.g., bats and mice) to several tons (e.g., elephants and whales), have resulted in great macroscopic variation in the morphology of mammalian limbs. For instance, the bones of the cetacean arm and forearm have adopted an hourglass-like shape to improve flipper resistance during swimming [9], while the femorotibial joint of elephants is far more extended and congruent in resting position than in other quadrupeds, to better support their large weight [10]. Interestingly, there are also adaptations on a smaller scale than the entire limb or joint. Mechanical properties of tissues depend both on the biochemical composition, and on ultrastructural organization. In the case of articular cartilage, the interaction of the collagen and proteoglycan networks is pivotal in this respect [11]. The study described in Chapter 2 analysed the adaptations of the osteochondral unit in relation to variation in body mass between species, showing that the different layers of articular cartilage (superficial, middle, and deep), scale in different ways. The earlier observation that overall cartilage thickness scaled with negative allometry [12] was confirmed, but the superficial and middle layer were shown to become relatively thinner in larger animals, while the deep zone adapted to increasing body mass isometrically. In terms of function, these findings support the hypothesis that the superficial layer has an important role in the distribution of forces, while the deep layer is more important for load absorption [13-15].

The analysis in Chapter 2 also offers insight into the biochemical changes in relation to scaling of cartilage, such as the relative concentrations of PGs in the different layers, allowing to put in a wider context the tissue's specialized mechanical behaviour. It has been previously described how the mechanical properties of cartilage are achieved through the interplay of the tissue's two main networks, one composed by its collagen fibres, and one by the proteoglycans interspersed in the matrix [11]. While the covalent crosslinks between the collagen fibres are crucial for providing tensile stiffness, the Donnan-osmotic pressure associated with the fixed charge density of the proteoglycans contributes to maintaining the collagen network under intrinsic tension [11]. As the deep layer tends to be richer in proteoglycans, this corresponds to a higher fixed charge density and higher osmotic pressure, increasing compressive stiffness. The quantification of normalized 
proteoglycan content across different species in Chapter 2 confirms the relatively higher concentration of PGs in the deep layer. Chapter 2 therefore shows that differences in scaling are accommodated by adaptations in thickness of the deep layer, rather than by modifications of the relative PG concentration.

In aquatic mammals, whose joints experience forces that are vastly different from terrestrial mammals, the thickness differences between layers were less marked, but the thickness of the superficial layer remained relatively constant $(11.31 \pm 7.4 \%$ in terrestrial, $12.30 \pm 5.5 \%$ in aquatic mammals, Chapter 3$)$. This similarity in the thickness of the superficial layer in species with widely different sizes, and living in different environments, points at the key role of this layer in distributing forces $[14,16]$. A role that should be recognized in the design of implants for the treatment of cartilage defects. In fact, from a functional perspective, hyaline articular cartilage tissue can be conceptually reduced to two zones, a superficial and a middle/deep layer. The superficial zone is meant to cope with tensile loads (principally generated by shear forces), to distribute compressive loads, and to enable frictionless motion between the bone ends; the middle/deep zone is responsible for the accommodation of compressive loads [17]. The distribution of loads by the superficial layer is enabled by the orientation and density of its collagen fibres. The tangential orientation of these fibres permits lateral distribution of axial loads, inducing tensile stress parallel to the surface, thus engaging a larger portion of the underlying tissue to support the load [13]. In aquatic mammals, the amount of collagen fibres with parallel orientation in the superficial layer is similar to that of terrestrial mammals, despite the difference of (compressive) impact loading experienced by these two groups. However, the collagen fibre alignment in the remainder of the aquatic mammals' cartilage appears to be essentially random, in contrast with the well characterized, depth-dependent collagen fibre organization displayed in terrestrial mammals. This significant difference in collagen network orientation confirms earlier findings in juvenile horses that the configuration of the fibres is driven by the experienced load patterns [18], and is reminiscent of the significance of the network's architecture in cartilage functionality. While the importance of collagen architecture has been long neglected in regenerative medicine of articular cartilage [19], the development of repair strategies is already veering towards this direction, as exemplified by the recent work of Castilho et al., where 
introducing a superficial tangential zone in engineered constructs, led to improved load-bearing, particularly under incongruent indentation [20]. Furthermore, the production of high density collagen regions approximating the articular cartilage zonal structure has been proven to induce cell alignment and phenotype conservation of primary chondrocytes [21]. While these applications are encouraging and indicative of the viability of the approximation of the native architecture as strategy for success, the concrete realization is not yet fully possible due to the limitations of current biofabrication technologies.

Regeneration of cartilage has not yet been achieved and the recapitulation of its zonal architecture is likely not enough to assure long-term functional repair: the osteochondral unit also encompasses the subchondral bone, and this structure and its interface with cartilage must be addressed in the design of scaffolds. Subchondral bone, in contrast to cartilage, is a dynamic tissue, as it retains the capacity to remodel throughout life [22], and it represents an endogenous source of regenerative cells and trophic factors [23]. The subchondral bone consists of the relatively compact subchondral plate and the trabecular bone below. It provides support and anchorage to cartilage and its trabecular part has an important role in the dampening of the forces exerted on the joint surface. Its absorbing ability is site-dependent and changes dynamically in response to imposed stress, therefore a certain degree of variation in density and thickness will be present, in correspondence to variation in mechanical properties [24, 25]. The subchondral plate acts as interface, distributing loads rather than carrying them, as confirmed in Chapter 2 by the absence of correlation of the subchondral plate thickness with body mass, contrary to the trabecular thickness of the trabecular bone below. This role of distribution of loading is further supported by the absence of a subchondral plate in aquatic mammals described in Chapter 3 . Here, there is a direct transition between cartilage and trabecular bone in absence of a calcified cartilage layer and a subchondral plate.

The differences found between terrestrial and aquatic mammals, and in the scaling of the components of the osteochondral unit, reflect the paramount importance of coping with loading for proper joint function. These differences are reflected in the essentially different configuration of the cartilage's collagen network in these two types of mammals and in the absence of an interface between articular cartilage and subchondral trabecular bone in 
marine mammals. They provide valuable clues towards the development of regenerative approaches for joint damage.

\section{Part II}

Joint regeneration: the long and perilous road from petri dish to patient

While in vitro regenerative approaches yield encouraging results, the complexity of the osteochondral unit has made the translation of regenerative strategies from bench to bedside a particularly hard task, as reflected by the scarce successful in vivo or clinical studies reported [26]. Many approaches that appear promising in the laboratory ultimately fail to meet the challenges of a preclinical or clinical setting [27]. As a result, despite the ample variety of hydrogel biomaterials that have been explored to support chondrogenesis in vitro, only few biomaterials make their way from the laboratory to the clinics [28-30].

The (large) animal model

- Ethical considerations

Already in 1825, the philosopher Jeremy Bentham, a well-known early advocate for animal rights, argued that the use of animal models could be acceptable, provided that the goal was a general benefit to humanity, that the chances of achieving that goal were reasonable, and that animals were not made to suffer unnecessarily [31]. In the late 1950s, the scientific community and general population called for further awareness of this issue, as attested by the publication of the book "The principle of humane experimental technique" by Russell and Burch, in which they first proposed the "Three R's" principle. These principles lay out guidance principles for the replacement of conscious living animals with non-sentient animals or materials, and advocate for the reduction of the number of animals used in an experiment or procedure, and refinement of the techniques used in order to decrease the incidence or amount of pain and distress [32]. Nowadays, the $3 \mathrm{R}$ principles have been incorporated into the pertinent regulations in many countries, particularly within the EU with the directive 2010/63/EU [33, 34]. These principles guided the criteria for the design of the studies described within this thesis, where for example the model was refined from having two defects 
within the same joint to one defect in each joint. And while successful results in osteochondral repair may be an ambitious goal in the short term, the refinement of the model should always be a priority in study design. Viable alternatives may be represented by advanced ex vivo or in vitro platforms which offer controlled environments to partly address the unknown issues before proceeding with further large animal studies [35, 36].

\section{- The equine model}

The use of the equine model raises ethical issues as any animal model, but these are often subjectively perceived as more impactful, based on the traditionally important role of horses in human society and the attraction many people feel towards horses, resulting in a privileged bond with humans. Another special feature of the horse is that the animal has been domesticated because of its locomotor system, rather than for the production of goods like meat or milk, making disorders of their locomotor system the clinically most important class of diseases. This, coupled with the often high economic value of the animal, has created a large clinical need for addressing these musculoskeletal disorders. This makes the horse, apart from an experimental animals into a target animals as well and hence into a model for its own joint diseases [37, 38].

The translation of new therapies to the equine model, brings some intrinsic challenges, as evidenced by the studies in Chapters 4 to 6 . One of the main limiting factors of the equine model is the immediate post-operative loading [39]. This is not representative of the human clinical recovery protocols, where full weight-bearing is usually avoided for a period of at least 6-8 weeks [40], but cannot be avoided in these animals. This challenge of early biomechanical loading on soft biomaterials, such as hydrogels, may lead to accelerated degradation or wear of the implant, as underscored by the results of Chapters 4 and 5 . As seen in the study discussed in Chapter 4, the use of a cell-based, GelMA hydrogel regeneration strategy did not yield the hypothesized positive results. When analysing possible causes of failure, it was concluded, based on the data available, that early degradation and insufficient fixation were the most probable causes. However, to which extent the early degradation could be attributed to the mechanical forces, to the immune cells, or to other factors, could not be uniquely identified due to the multitude of factors at 
play.

Longitudinal monitoring in long-term in vivo models is fundamental, as subtle changes that occur over a long period of time may be otherwise difficult to detect. If possible, quantitative parameters should be used. An example is the functional assessment through quantitative gait analysis measuring kinetic and/or kinematic parameters, which is an excellent tool for quantification of the functional outcome over time, particularly with the rapid development of the techniques and modelling reported in the last years [41, 42]. Indeed, quantitative gait analysis appeared to be more sensitive for the detection of subtle gait anomalies than the classic visual subjective assessment by an experienced clinician in Chapters 4 and 6. Longitudinal monitoring allows also to observe and quantify the evolution of the repair tissue over time, for which the equine model lends itself very well. For example, the use of second-look arthroscopy allows for simultaneous use of advanced imaging techniques, such as optical coherence tomography [43] or near infrared spectroscopy [44]. A disadvantage is that this still is a minimally invasive intervention, which carries intrinsic costs and risks. Imaging options can also include radiography and ultrasonography, which are less invasive and costly, or magnetic resonance imaging or computed tomography, with and without the aid of contrast agents, although these require general anaesthesia of the animals in order to be performed.

\section{- Fixation in chondral defects in the equine model}

In specific cases where damage is limited to small areas, chondral defects are typically treated by debridement only, as use of micro-fracturing would imply breaching the otherwise healthy bone-cartilage interface, which carries the risk of creating morbidity and hence affecting joint function [45, 46]. An ideal solution would be using a technique that would permit filling the defect and restoring cartilage tissue, without further damage to the subchondral bone.

Biological glues or the direct casting of hydrogels into defects could theoretically be suitable for such an approach, however they may be too weak to prevent early detachment of implants, particularly in joint areas subjected to harsh loading regimens. This may have been the case in Chapter 4 , where GelMA was directly cast into the defect, and was simultaneously used as fixation for the 3D-printed microfibre meshes, which were gone two months after implantation. Additionally, manual direct casting, strongly limits the 
possibility to add complexity to the architecture of repair scaffolds. In Chapter 5 , fibrin glue was also used as a fixative, with results that were considerably worse than those of an osteochondral press-fit solution. An additional issue is that the use of biological glues may be hampered by the unavailability of species-specific components, as further discussed in Chapter 5.

The limited thickness of 1-2 mm of cartilage commonly found in the knee (stifle) joints of both humans and horses [47], along with the composition of the tissue, limits the fixation options without using the bone underneath for anchoring. In fact, with the currently available techniques, adequate fixation of implants or scaffolds in a partial thickness cartilage defect is not feasible, particularly not in the equine model in which loading occurs immediately after surgery. Chapter 5 concludes that the press-fit placed osteochondral construct is the safer strategy. Sealing hydrogels that attach firmly to the native tissue and have biomechanical properties matching those of articular cartilage might, however, be an alternative $[48,49]$.

In vivo cartilage repair: importance of the replication of the zonal architecture

\section{- Challenges in translation}

In the studies described in Part II of this thesis (Chapters 4-6), different regenerative strategies have been tested in the equine model [39]. Long-term studies are necessary to obtain answers to the predetermined research questions, ultimately providing information on the efficacy of a regenerative strategy. However, in order to obtain insight into the dynamics of a repair approach, shorter term studies should not be overlooked, and while long-term evaluation is necessary to avoid overestimation of the potential of a repair strategy [27], short-term pilots with a tailored duration may be able for example to provide relevant information on material degradation. As described in Chapters 4 and 5, 2-week pilots confirmed the presence of hydrogel-based implants in terms of fixation. However, as suggested by the lack of the reinforcing microfibre meshes 2 months after implantation, early degradation may have occurred somewhere between 2 and 8 weeks. Conversely, the intermediate 4 week duration of the pilots described in Chapter 5, seemed to highlight the degradation occurring in hydrogel implants, suggesting that a 2-week pilot, while sufficient to detect fixation issues, may 
not be sufficient to detect early hydrogel degradation.

From a regenerative perspective, the success of an implant is also dependent on its capacity to either deliver or recruit cells that will, in time, deposit new tissue. The use of hydrogels permits the encapsulation and delivery of cells, however environmental differentiation conditions may be less optimal than the ones investigated in vitro. Moreover, the harsh biomechanical environment of the in vivo equine joint, further challenges the performance of the regenerative implants, as illustrated by the studies described in Part II of this thesis. The limited repair observed with the hydrogel-based implants in these studies, may have been due to the early degradation of the hydrogel, which could have resulted in the early exposure of cells while sufficient new matrix deposition was not yet achieved. An alternative route to partially address this limitation, would be the pre-culturing of the scaffolds, which would enable cells to benefit from the optimal in vitro conditions to initiate the deposition of cartilage-like matrix.

\section{- Towards the replication of the zonal architecture}

Some of the techniques for (osteo)chondral repair currently applied in the clinics rely on a relatively simple approach that aims at kick-starting the regenerative process within the patient, as is done for example with microfracture $[50,51]$. Conversely, novel tissue-engineering based strategies aim to reproduce, at least partly, the features of the native osteochondral tissue, by fabricating advanced composite constructs (Chapter 6 and Chapter 7). In these studies, constructs were constituted of a chondral portion filled with two layers of a HA-based (or StarPEG-based in Chapter 7) hydrogel cellladen with two different cell types with 3D-printed reinforcing poly $(\varepsilon$ caprolactone) (PCL) fibres integrated to a PCL bone anchor. After 6 months of implantation, the constructs resulted in good filling of the defects, associated with a good integration between the scaffold-cartilage interface, which can prevent further deterioration of the native tissue [52]. The tissue that was formed, however, lacked the desired biomechanical and biochemical characteristics of native cartilage. Fibre-reinforcement can improve the stiffness of hydrogels [53], however it only constitutes one of many steps towards the approximation of the native tissue's properties. As the integrity of the subchondral bone, and with it the interface between bone and cartilage, is 
fundamental for physiological function, in an osteochondral construct the integration between the chondral and osteal portion should be carefully addressed. Due to the widely differing characteristics of bone and cartilage tissues, these two elements are usually reproduced with different technologies and materials, such as osteoinductive materials for the bone portion, and cellfriendly hydrogels for the cartilage portion [54].

For the fabrication of the osteochondral scaffolds described in Part II of this thesis, first the osteal portion of the anchor was 3D-printed, with protruding fibre reinforcement to enhance hydrogel fixation. This portion had a diminishing porosity from bottom to top (Chapter 5), and an interface virtually non-porous to cells. Subsequently, a hydrogel layer was cast directly on top of the anchor, infusing the PCL-reinforced area. This multi-step process is laborious and therefore less appealing for translation in a clinical setting where manufacturing in a sterile, GMP (Good Manufacturing Practice) conformed environment is mandatory. However, recent developments in biofabrication have shown the potential of the convergence of multiple techniques, like the combination of extrusion-based bioprinting and melt electrowriting to print cell-encapsulated hydrogels and microfibres in a single-step fabrication process [55].

Technologies such as this are quickly evolving: 3D-printers are able to print smaller and smaller fibres, and new bio-inks are being developed, paving the road for the future mimicking of the functional properties of the collagen network. Besides extrusion-based 3D printing of cell laden gels [56], novel methodologies include solution electrospinning of fibre materials [57] and fabrication of particulate templates of porous polymeric structures [58]. The use of these novel technologies allowed, for example, the bioengineering of a microfiber-reinforced hydrogel construct that replicated the zonal depthdependent mechanical properties of native cartilage, and upon culture under mechanical conditioning, resulted in neo-cartilage formation [20]. While this work is extremely promising, long-term evaluation in a large animal model is still needed for the final proof whether the strategy yields successful (osteo)chondral repair or not.

\section{The path to human and veterinary patients}

If successful in large animal models, the translation of advanced products for (osteo)chondral repair requires meeting a series of regulatory requirements to 
ultimately reach the human patient. In the US, a simple 3D-printed implant without cells or chemical cues is considered by the FDA (US Food and Drug Administration) a medical device. The osteochondral anchor described In Part II, if stripped of hydrogel or cells, would fall in this category, as it is a healthcare product that does not require chemical action or metabolization to achieve its purpose [30]. When biological components are included, the product is categorized as an advanced therapy medicinal product (ATMP). Examples of ATMPs already available in the clinics are products for $\mathrm{ACl}$ and $\mathrm{MACl}$, currently approved for the repair of focal cartilage defects $[59,60]$. To obtain ATMP certification/approval, a product's efficacy, quality and safety must be demonstrated. Additionally, such constructs should be manufactured in compliance with the requirements for GMP [59].

In Europe, the regulatory body is the European Medicines Agency (EMA), which published its first guideline on human cell-based medicinal products in 2008 [61]. Since regenerative medicine is a relatively new field, few strategies have successfully reached the preclinical stage, and the legislation on safety requirements and processes has had to catch up with technological developments, resulting in a regulatory iter that is not always clearly defined. In recent years, the EMA has started providing support to industries interested in the development of novel strategies through a service of "scientific advice" that supports the development of high-quality, effective safe medicines. It may be emblematic for the importance of joint disease that the first-ever product of the category of regenerative medicine recommended for marketing in veterinary medicine by the EMA, was a stem-cell based therapy for the treatment of lameness related to joint inflammation in the horse [62].

While the complexity of the regulatory iter may seem far away from the bench in the research lab, it should be kept in mind that the simpler the approach, the easier translatable. In this sense, the evolution of technologies that allow, for example, the convergence of multiple 3D-printing techniques [55], represents a promising opportunity.

\section{Conclusions and future perspectives}

The osteochondral unit is a functional entity composed by specialized tissues that enable it to function as a key structural element exposed to a biomechanically challenging environment. To date, the understanding of these tissues from a biological and mechanical point of view is incomplete, as 
attested by the continuous novel findings reported on the fundamental science of both bone and cartilage tissues.

The use of different techniques from the fields of tissue engineering and biofabrication may enable us to advance toward the production of lab-made regenerative implants. This multidisciplinary effort may finally bring us closer to approximating the native tissues within the joint. The insight into the role of structural elements of the osteochondral unit, including the collagen network and the subchondral bone plate, as reported in this thesis, will aid in the design of durable implants for a functional joint replacement.

Here, significant improvements were made towards the translation of regenerative strategies for osteochondral repair. Chondral and osteochondral defect models in the horse were refined and the need for proper fixation of regenerative implants was addressed. Yet, more hurdles must be overcome to obtain a success with scaffolds for (osteo)chondral repair in vivo. Given the lessons learned in Part I, the integration between the phases replicating cartilage and bone will be a challenging yet promising area of focus for future research. Given the lessons learned in Part II, there is a need for tailoring of the degradation of hydrogels, and, more in general, for improving the functionality and durability of biofabricated osteochondral scaffolds. In this sense, it may well be that accurately replicating the functional (biomechanical) aspects of the tissue's architecture is the key to success. 


\section{REFERENCES}

[1] T.W.B. Group, Life expectancy at birth, total (years), 2020. https://data.worldbank.org/ indicator/SP.DYN.LEOO.IN.

[2] C. Cardona, D. Bishai, The slowing pace of life expectancy gains since 1950, BMC public health 18(1) (2018) 151.

[3] P.K. Linden, History of solid organ transplantation and organ donation, Critical care clinics 25(1) (2009) 165-184.

[4] Z.M. Jessop, A. Al-Sabah, W.R. Francis, I.S. Whitaker, Transforming healthcare through regenerative medicine, BMC Medicine 14(1) (2016) 115.

[5] A.D. Woolf, B. Pfleger, Burden of major musculoskeletal conditions, Bulletin of the world health organization 81 (2003) 646-656.

[6] Y. Krishnan, A.J. Grodzinsky, Cartilage diseases, Matrix Biology 71 (2018) 51-69.

[7] S.C. Mastbergen, D.B. Saris, F.P. Lafeber, Functional articular cartilage repair: here, near, or is the best approach not yet clear?, Nature reviews rheumatology 9(5) (2013) 277.

[8] A.J. Sophia Fox, A. Bedi, S.A. Rodeo, The basic science of articular cartilage: structure, composition, and function, Sports health 1(6) (2009) 461-468.

[9] M.A. Taylor, Functional significance of bone ballastin in the evolution of buoyancy control strategies by aquatic tetrapods, Historical Biology 14(1-2) (2000) 15-31.

[10] G. Weissengruber, F. Fuss, G. Egger, G. Stanek, K. Hittmair, G. Forstenpointner, The elephant knee joint: morphological and biomechanical considerations, Journal of anatomy 208(1) (2006) 59-72.

[11] V.C. Mow, A. Ratcliffe, A.R. Poole, Cartilage and diarthrodial joints as paradigms for hierarchical materials and structures, Biomaterials 13(2) (1992) 67-97.

[12] J. Malda, J.C. de Grauw, K.E. Benders, M.J. Kik, C.H. van de Lest, L.B. Creemers, W.J. Dhert, P.R. van Weeren, Of mice, men and elephants: the relation between articular cartilage thickness and body mass, PloS one 8(2) (2013).

[13] C. Glaser, R. Putz, Functional anatomy of articular cartilage under compressive loading Quantitative aspects of global, local and zonal reactions of the collagenous network with respect to the surface integrity, Osteoarthritis and cartilage 10(2) (2002) 83-99.

[14] S. Bevill, A. Thambyah, N. Broom, New insights into the role of the superficial tangential zone in influencing the microstructural response of articular cartilage to compression, Osteoarthritis and Cartilage 18(10) (2010) 1310-1318.

[15] R. Korhonen, M. Wong, J. Arokoski, R. Lindgren, H. Helminen, E. Hunziker, J. Jurvelin, Importance of the superficial tissue layer for the indentation stiffness of articular cartilage, Medical engineering \& physics 24(2) (2002) 99-108.

[16] H. Muir, P. Bullough, A. Maroudas, The distribution of collagen in human articular cartilage with some of its physiological implications, The Journal of bone and joint surgery. British volume 52(3) (1970) 554-563.

[17] S. Camarero-Espinosa, B. Rothen-Rutishauser, E.J. Foster, C. Weder, Articular cartilage: from formation to tissue engineering, Biomaterials science 4(5) (2016) 734-767.

[18] P.A. Brama, J. Holopainen, P.R. van Weeren, E.C. Firth, H.J. Helminen, M.M. Hyttinen, Effect of loading on the organization of the collagen fibril network in juvenile equine articular cartilage, Journal of Orthopaedic Research 27(9) (2009) 1226-1234.

[19] J. Malda, J. Groll, P.R. van Weeren, Rethinking articular cartilage regeneration based on a 250-year-old statement, Nature Reviews Rheumatology (15) (2019) 571-572.

[20] M. Castilho, V. Mouser, M. Chen, J. Malda, K. Ito, Bi-layered micro-fibre reinforced hydrogels for articular cartilage regeneration, Acta biomaterialia 95 (2019) 297-306.

[21] T. Novak, B. Seelbinder, C.M. Twitchell, C.C. van Donkelaar, S.L. Voytik-Harbin, C.P. Neu, Mechanisms and microenvironment investigation of cellularized high density gradient collagen matrices via densification, Advanced functional materials 26(16) (2016) 2617-2628.

[22] H.L. Stewart, C.E. Kawcak, The importance of subchondral bone in the pathophysiology of osteoarthritis, Frontiers in veterinary science 5 (2018) 178. 
[23] H. Madry, C.N. van Dijk, M. Mueller-Gerbl, The basic science of the subchondral bone, Knee surgery, sports traumatology, arthroscopy 18(4) (2010) 419-433.

[24] P.A. Simkin, T.F. Heston, D.J. Downey, R.S. Benedict, H.S. Choi, Subchondral architecture in bones of the canine shoulder, Journal of anatomy 175 (1991) 213.

[25] P.A. Simkin, S.J. Houglum, C.C. Pickerell, Compliance and viscoelasticity of canine shoulders loaded in vitro, Journal of biomechanics 18(10) (1985) 735-743.

[26] A. Armiento, M. Stoddart, M. Alini, D. Eglin, Biomaterials for articular cartilage tissue engineering: Learning from biology, Acta biomaterialia 65 (2018) 1-20.

[27] R.V. Bolaños, S. Cokelaere, J.E. McDermott, K. Benders, U. Gbureck, S. Plomp, H. Weinans, J. Groll, P. van Weeren, J. Malda, The use of a cartilage decellularized matrix scaffold for the repair of osteochondral defects: the importance of long-term studies in a large animal model, Osteoarthritis and Cartilage 25(3) (2017) 413-420.

[28] K. Sadtler, A. Singh, M.T. Wolf, X. Wang, D.M. Pardoll, J.H. Elisseeff, Design, clinical translation and immunological response of biomaterials in regenerative medicine, Nature Reviews Materials 1(7) (2016) 1-17.

[29] S.J. Hollister, W.L. Murphy, Scaffold translation: barriers between concept and clinic, Tissue Engineering Part B: Reviews 17(6) (2011) 459-474.

[30] M.J. Webber, O.F. Khan, S.A. Sydlik, B.C. Tang, R. Langer, A perspective on the clinical translation of scaffolds for tissue engineering, Annals of biomedical engineering 43(3) (2015) 641-656.

[31] J. Kniess, Bentham on animal welfare, British Journal for the History of Philosophy 27(3) (2019) 556-572.

[32] L.J. Bishop, A.L. Nolen, Animals in research and education: Ethical issues, Kennedy Institute of Ethics Journal 11(1) (2001) 91-112.

[33] I.A.S. Olsson, S.P.d. Silva, D. Townend, P. Sandøe, Protecting animals and enabling research in the European Union: An overview of development and implementation of directive 2010/63/ EU, ILAR journal 57(3) (2017) 347-357.

[34] E. Parliament, Regulation (EU) 2019/1010 of the European Parliament and of the Council of 5 June 2019, 2019. https://eur-lex.europa.eu/legal-content/EN/TXT/?uri=CELEX:02010L006320190626. (Accessed $18-6-2020$ 2020).

[35] A. Schwab, A. Meeuwsen, F. Ehlicke, J. Hansmann, L. Mulder, A. Smits, H. Walles, L. Kock, Ex vivo culture platform for assessment of cartilage repair treatment strategies, ALTEX-Alternatives to animal experimentation 34(2) (2017) 267-277.

[36] S. Piluso, Y. Li, F. Abinzano, R. Levato, L.M. Teixeira, M. Karperien, J. Leijten, R. van Weeren, J. Malda, Mimicking the articular joint with in vitro models, Trends in biotechnology 37(10) (2019) 1063-1077.

[37] C.W. Mcllwraith, D.D. Frisbie, C.E. Kawcak, R. Van Weeren, Joint Disease in the Horse-E-Book, Elsevier Health Sciences2015.

[38] C. Mcllwraith, D. Frisbie, C. Kawcak, The horse as a model of naturally occurring osteoarthritis, Bone \& joint research 1(11) (2012) 297-309.

[39] C.W. Mcllwraith, L.A. Fortier, D.D. Frisbie, A.J. Nixon, Equine models of articular cartilage repair, Cartilage 2(4) (2011) 317-326.

[40] M.J. Kraeutler, J.W. Belk, T.J. Carver, E.C. McCarty, Is delayed weightbearing after matrixassociated autologous chondrocyte implantation in the knee associated with better outcomes? A systematic review of randomized controlled trials, Orthopaedic journal of sports medicine 6(5) (2018) 2325967118770986.

[41] P. van Weeren, C. Gómez Álvarez, Equine gait analysis: The slow start, the recent breakthroughs and the sky as the limit?, Equine veterinary journal 51(6) (2019) 809-810.

[42] F.S. Bragança, M. Rhodin, P. van Weeren, On the brink of daily clinical application of objective gait analysis: What evidence do we have so far from studies using an induced lameness model?, The Veterinary Journal 234 (2018) 11-23.

[43] T. Niemelä, T. Virén, J. Liukkonen, D. Argüelles, N.C. te Moller, P.H. Puhakka, J.S. Jurvelin, R.M. Tulamo, J. Töyräs, Application of optical coherence tomography enhances reproducibility of arthroscopic evaluation of equine joints, Acta veterinaria scandinavica 56(1) (2014) 3. 
[44] J.K. Sarin, N.C. Te Moller, I.A. Mancini, H. Brommer, J. Visser, J. Malda, P.R. van Weeren, I.O. Afara, J. Töyräs, Arthroscopic near infrared spectroscopy enables simultaneous quantitative evaluation of articular cartilage and subchondral bone in vivo, Scientific reports 8(1) (2018) 1-10.

[45] C.E. Kawcak, C.W. Mcllwraith, R. Norrdin, R. Park, S. James, The role of subchondral bone in joint disease: a review, Equine veterinary journal 33(2) (2001) 120-126.

[46] S.R. Simon, E.L. Radin, I.L. Paul, R.M. Rose, The response of joints to impact loading - II In vivo behavior of subchondral bone, Journal of Biomechanics 5(3) (1972) 267-272.

[47] J. Malda, K. Benders, T. Klein, J. De Grauw, M. Kik, D. Hutmacher, D. Saris, P. Van Weeren, W. Dhert, Comparative study of depth-dependent characteristics of equine and human osteochondral tissue from the medial and lateral femoral condyles, Osteoarthritis and cartilage 20(10) (2012) 1147-1151.

[48] R. Jin, L.M. Teixeira, P.J. Dijkstra, C. Van Blitterswijk, M. Karperien, J. Feijen, Enzymaticallycrosslinked injectable hydrogels based on biomimetic dextran-hyaluronic acid conjugates for cartilage tissue engineering, Biomaterials 31(11) (2010) 3103-3113.

[49] R. Wang, N. Leber, C. Buhl, N. Verdonschot, P.J. Dijkstra, M. Karperien, Cartilage adhesive and mechanical properties of enzymatically crosslinked polysaccharide tyramine conjugate hydrogels, Polymers for advanced technologies 25(5) (2014) 568-574.

[50] P. Kreuz, M. Steinwachs, C. Erggelet, S. Krause, G. Konrad, M. Uhl, N. Südkamp, Results after microfracture of full-thickness chondral defects in different compartments in the knee, Osteoarthritis and cartilage 14(11) (2006) 1119-1125.

[51] J.R. Steadman, W.G. Rodkey, J.J. Rodrigo, Microfracture: surgical technique and rehabilitation to treat chondral defects, Clinical Orthopaedics and Related Research ${ }^{\circledR} 391$ (2001) S362-S369.

[52] D.D. Frisbie, H.E. McCarthy, C.W. Archer, M.F. Barrett, C.W. Mcllwraith, Evaluation of articular cartilage progenitor cells for the repair of articular defects in an equine model, JBJS 97(6) (2015) 484-493.

[53] J. Visser, F.P. Melchels, J.E. Jeon, E.M. Van Bussel, L.S. Kimpton, H.M. Byrne, W.J. Dhert, P.D. Dalton, D.W. Hutmacher, J. Malda, Reinforcement of hydrogels using three-dimensionally printed microfibres, Nature communications 6(1) (2015) 1-10.

[54] W.M. Groen, P. Diloksumpan, P.R. van Weeren, R. Levato, J. Malda, From intricate to integrated: Biofabrication of articulating joints, Journal of Orthopaedic Research 35(10) (2017) 2089-2097.

[55] M. de Ruijter, A. Ribeiro, I. Dokter, M. Castilho, J. Malda, Simultaneous micropatterning of fibrous meshes and bioinks for the fabrication of living tissue constructs, Advanced healthcare materials 8(7) (2019) 1800418.

[56] X. Ren, F. Wang, C. Chen, X. Gong, L. Yin, L. Yang, Engineering zonal cartilage through bioprinting collagen type II hydrogel constructs with biomimetic chondrocyte density gradient, BMC musculoskeletal disorders 17(1) (2016) 301.

[57] H. Chen, A.d.B.F.B. Malheiro, C. van Blitterswijk, C. Mota, P.A. Wieringa, L. Moroni, Direct writing electrospinning of scaffolds with multidimensional fiber architecture for hierarchical tissue engineering, ACS applied materials \& interfaces 9(44) (2017) 38187-38200.

[58] J. Steele, S. McCullen, A. Callanan, H. Autefage, M. Accardi, D. Dini, M. Stevens, Combinatorial scaffold morphologies for zonal articular cartilage engineering, Acta biomaterialia 10(5) (2014) 2065-2075.

[59] K.F. Pearce, M. Hildebrandt, H. Greinix, S. Scheding, U. Koehl, N. Worel, J. Apperley, M. Edinger, A. Hauser, E. Mischak-Weissinger, Regulation of advanced therapy medicinal products in Europe and the role of academia, Cytotherapy 16(3) (2014) 289-297.

[60] E. Parliament, t.C.o.t.E. Union, Regulation (EC) No 1394/2007 of the European Parliament and of the Council of 13 November 2007 on advanced therapy medicinal products and amending Directive 2001/83/EC and Regulation (EC) No 726/2004, Official Journal 324 (2007) 121-137.

[61] C.f.m.p.f.h.u. /EMA, Guideline on human cell-based medicinal products, European Medicines Agency, London, United Kingdom, 2008.

[62] C.f.M.P.f.V. Use/EMA, First stem cell-based veterinary medicine recommended for marketing authorisation European Medicines Agency, 2018. 


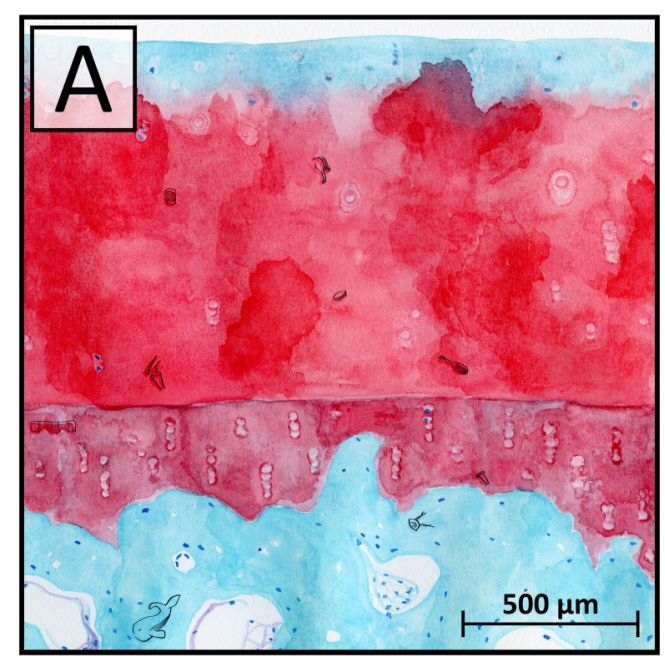




\section{Summaries}

Nederlandse Samenvatting

Riassunto in italiano

Résumé en français 


\section{NEDERLANDSE SAMENVATTING}

Dit proefschrift richt zich op de ontwikkeling van strategieën voor translationeel onderzoek op het gebied van therapieontwikkeling voor de behandeling van osteochondrale defecten in gewrichten. Dit wordt gedaan door middel van vergelijkende studies over meerdere diersoorten naar de natuurlijke configuratie van de functionele eenheid die gevormd wordt door het gewrichtskraakbeen en het onderliggende subchondrale bot, en door een verfijning van het paardenmodel.

De bestudering van de basale biologie van de osteochondrale functionele eenheid bij meer dan 40 verschillende zoogdiersoorten levert informatie op over de hoe de natuur omgaat met het accommoderen van de op gewrichten uitgeoefende krachten. Dit begrip is essentieel voor het ontwikkelen van succesvolle regeneratieve therapieën voor het behandelen van gewrichtsletsel.

De translatie van potentiële nieuwe therapieën die veelbelovend zijn in in vitro onderzoek naar het preklinische paardenmodel wordt belemmerd door het gebrek aan een effectieve en betrouwbare methode om implantaten in het gewricht vast te zetten. In dit proefschrift wordt dit probleem aangepakt door het model te verfijnen met de introductie van een geavanceerd samengesteld implantaat dat in het onder het kraakbeen liggende bot verankerd kan worden.

\section{Deel I}

Gewrichtskraakbeen is een sterk gespecialiseerd weefsel dat gezien moet worden in de context van de hele osteochondrale functionele eenheid. In Hoofdstuk 2 wordt er gekeken naar de relatie tussen lichaamsgewicht en verschillende structurele elementen van de osteochondrale functionele eenheid. Deze vergelijkende studie bij 37 zoogdiersoorten laat zien dat, waar de relatie tussen lichaamsgewicht en totale kraakbeendikte negatief allometrisch is, dat niet geldt voor alle afzonderlijke lagen in het kraakbeen. De aanpassing aan een groter lichaamsgewicht vindt vooral plaats in de diepe laag van het hyaliene gewrichtskraakbeen waarbij de oppervlakkige laag relatief dunner wordt. De dikte van de subchondrale plaat en het totale volume aan bot zijn niet gerelateerd aan lichaamsgewicht, de dikte van de beenbalkjes van het daaronder gelegen bot heeft wel een duidelijke positieve relatie met lichaamsgewicht $(r=0.75, p<0.001)$. Deze relatie was verwacht, omdat het 
bekend is dat dit weefsel een belangrijke rol heeft in het opvangen van de belasting. Op basis van de studie kan geconcludeerd worden dat gewrichtskraakbeen heel geconserveerd is binnen vele diersoorten en dat het vooral het subchondrale bot is dat zich aanpast aan het lichaamsgewicht. Deze onveranderlijkheid van gewrichtskraakbeen is een eigenschap die meegenomen dient te worden bij het ontwerpen van regeneratieve strategieën.

In Hoofdstuk 3 wordt onderzoek gedaan naar de osteochondrale functionele eenheid van een aantal soorten land- en zeezoogdieren met uiteenlopend lichaamsgewicht. Hierbij wordt speciale aandacht gegeven aan de microarchitectuur en de biomechanische eigenschappen van die eenheid, die sleutelelementen zijn voor de functie van het gewricht. Zeezoogdieren vormen een unieke groep dieren omdat ze, na de oorspronkelijke evolutie in het water net als alle diersoorten, landbewoners geworden zijn en daarna weer teruggekeerd zijn naar het marine milieu. Het kan dus worden verwacht dat de oorspronkelijke aanpassingen aan het landleven weer (gedeeltelijk) een andere kant op geëvolueerd zijn nadat deze soorten weer naar hun oorspronkelijke aquatische milieu gteruggekeerd zijn. Dat milieu wordt uiteraard gekenmerkt door een geheel andere belastingssituatie door het ontbreken van de invloed van de zwaartekracht.

Het onderzoek wijst uit dat het gewrichtskraakbeen van zeezoogdieren niet de kenmerkende boogstructuur van het collageennetwerk van de extracellulaire matrix heeft zoals die bij landdieren gezien wordt. Terwijl er bij landdieren een duidelijke, per kraakbeenzone verschillende, ordening van de collageenfibrillen bestaat, ontbreekt deze bij de zeezoogdieren en is de verdeling van de fibrillen willekeurig. Ook hebben zeezoogdieren geen gecalcifeerd kraakbeen onder het hyaliene kraakbeen, zoals dat bij alle landdieren gezien wordt, en is de subchondrale plaat heel dun en poreus. In biomechanisch opzicht is de maximale elastische modulus lager bij zeezoogdieren en is het kraakbeen minder stijf. De bevindingen in dit onderzoek leveren het natuurlijke bewijs van de grote invloed van belasting op het weefsel van de osteochondrale functionele eenheid en benadrukken dus ook het grote belang van het kunnen accommoderen van die belasting voor een goede gewrichtsfunctie. 


\section{Deel II}

Cellulaire therapieën voor de behandeling van gewrichtskraakbeendefecten maken tot nu toe voornamelijk gebruik van dragermaterialen voor die cellen die snel geresorbeerd worden, zoals fibrinelijm. Maar ook het gebruik van hydrogelen voor dit doel wordt onderzocht vanwege het feit dat die in chemische zin makkelijk aangepast kunnen worden en ook gefunctionaliseerd kunnen worden met biologisch actieve stoffen. In Hoofdstuk 4 wordt de hydrogel Gelatine Methacryloyl (GelMA), waarvan bij voorafgaand in vitro onderzoek aangetoond was dat er bij kweek van kraakbeencellen in dit materiaal een goede productie door die cellen van bestanddelen van de extracellulaire matrix is, toegepast in het paardenmodel. Na ontwikkeling van de chirurgische implantatietechniek wordt het materiaal eerst bij één Shetland pony toegepast. De implantatietechniek blijkt goed te werken en het blijkt dat het geïmplanteerde materiaal, met en zonder versterking in de vorm van een kunstmatig netwerk, goed blijft zitten. Naar aanleiding hiervan wordt er een studie van 12 maanden opgestart met 8 Shetland ponies waarbij GelMA (met en zonder versteviging) ingezaaid wordt met een mengsel van chondronen (chondrocyten clusters) en mesenchymale stamcellen. Ondanks de goede resultaten van de eerdere pilot study blijkt echter bij arthroscopisch onderzoek door middel van optische coherentie tomografie (OCT) op twee maanden na implantatie dat de verstevigingsnetjes niet meer aanwezig zijn. Deze bevinding wordt bevestigd door histologisch onderzoek op 12 maanden aan het einde van het experiment. Daarbij blijkt ook dat er geen kraakbeen, maar fibreus littekenweefsel gevormd is. Het heeft er dus alle schijn van dat bij dit onderzoek er sprake is geweest van een (te) snelle afbraak van de hydrogel en een onvoldoende fixatie van het implantaat, wat geleid heeft tot een vorm van weefselherstel die ook bij onbehandelde defecten gezien wordt.

Hoofdstuk $\mathbf{5}$ is gewijd aan het fixatieprobleem van chondrale implantaten. In eerste instantie wordt commercieel verkrijgbare fibrinelijm (die veel humane componenten bevat) vergeleken met diersoortspecifieke fribrinelijm die (grotendeels) gemaakt wordt met van het paard afkomstige componenten. Het commerciële product blijkt tot een ernstige immuunreactie bij het paard te leiden. De autologe vorm lost dit probleem maar gedeeltelijk op. Ten eerste is de productie complex en ten tweede is er nog steeds een (minder ernstige) immuunrespons, waarschijnlijk omdat er nog steeds enige componenten gebruikt worden die niet van het paard afkomstig zijn. Er wordt een alternatief 
ontwikkeld in de vorm van een osteochondrale plug die uit een basis van polycaprolactone (PCL) bestaat die press-fit in een osteochondraal defect geïmplanteerd kan worden en waarvan het chondrale deel uit verschillende te testen materialen kan bestaan.

In de Hoofdstukken $\mathbf{6}$ en $\mathbf{7}$ wordt de plug die ontwikkeld is in Hoofdstuk 5 gebruikt om de effecten van een zonale opbouw van het chondrale deel van het implantaat uit te testen in het osteochondrale paardenmodel. Daartoe wordt de PCL plug voorzien van twee verschillende chondrale delen. De ene bestaat uit twee lagen van een thiol-ene cross-linkable hyaluronic acid/ poly (glycidol) hybrid hydrogel (HA-SH / P(AGE-CO-G) (Hoofdstuk 6), de ander uit twee lagen StarPEG/heparin (Hoofdstuk 7). In beide gevallen bevat de bovenste laag specifieke chondrale stamcellen (Articular Cartilage Progenitor Cells / ACPCS) en de onderste de meer aspecifieke mesenchymale stamcellen (MSCs). De contrôle-implantaten hebben een uniforme populatie van MSCs. Na 6 maanden implantatie is er sprake van een goede botingroei in de PCL basis van het implantaat, maar er is maar een beperkte vorming van kraakbeenachtig weefsel in het chondrale deel van het implantaat. Daar kunnen verschillende redenen voor zijn, zoals een snelle afbraak van de hydrogel of een (te) vroeg verlies van de geïmplanteerde cellen. De StarPEG (Hoofdstuk 7) degradeert minder snel dan de HA-SH/P(AGE-co-G) hydrogel, maar ook daar is er sprake van een beperkte weefselformatie, deze is vergelijkbaar met die in Hoofdstuk 6. 


\section{RIASSUNTO IN ITALIANO}

Questa tesi è incentrata sullo sviluppo di strategie traslazionali per la rigenerazione di difetti (osteo)condrali tramite il perfezionamento del modello equino e lo studio morfologico e strutturale dell'unitá osteocondrale.

Attraverso l'analisi della biologia di base dell'unità osteocondrale di oltre 40 specie di mammifero, vengono chiarificati i meccanismi adottati dall'unità per far fronte alle diverse masse corporee e profili di carico. L'individuazione e la comprensione di questi meccanismi sono fondamentali per la pianificazione di una strategia terapeutica osteocondrale di successo.

La traslazione verso il modello preclinico equino di promettenti strategie rigenerative in vitro volte alla cura dei difetti osteocondrali è, attualmente, ostacolata dalla mancanza di tecniche affidabili ed efficaci per la fissazione degli impianti. Parte del lavoro qui descritto affronta questa mancanza, perfezionando il modello equino: partendo da un approccio puramente condrale, viene adottata una strategia più complessa in cui viene utilizzata uno scaffold composito adatto alla cura di difetti osteocondrali di dimensioni maggiori.

\section{Parte I}

La cartilagine articolare è un tessuto con un alto grado di specializzazione che, dal punto di vista funzionale, può essere meglio apprezzato nel contesto più ampio di unità osteocondrale. II capitolo $\mathbf{2}$ analizza i meccanismi di adattamento delle dimensioni degli elementi strutturali che compongono l'unità osteocondrale in un gruppo di animali con un'ampia variazione di taglia. L'analisi comparativa compiuta su osso e cartilagine di 37 specie diverse di mammifero dimostra che, mentre lo spessore totale della cartilagine scala rispetto alla massa corporea con una relazione allometrica negativa, i diversi strati della cartilagine non seguono lo stesso comportamento. Gli strati cartilaginei scalano in relazione ad un aumento di massa principalmente nello strato profondo, mentre lo strato superficiale dimostra di diventare relativamente più sottile. Lo spessore della placca ossea subcondrale e la frazione di volume osseo dimostrano di non avere correlazione con la massa corporea. Al contrario, l'osso trabecolare risulta avere trabecole relativamente più spesse in animali di dimensioni maggiori $(r=0.75, p<0.001)$. Tale tendenza è da aspettarsi se si considera che questa struttura supporta la maggior parte dei 
carichi ed è coinvolta nella mitigazione delle forze. È possibile concludere, da questa analisi, che la struttura del tessuto osteocondrale è essenzialmente conservata nelle diverse specie mammifere, e che i meccanismi principali di compensazione alla massa corporea risiedono nell'osso trabecolare. In aggiunta, l'immutevolezza e costanza del tessuto cartilagineo articolare andrebbero presi in considerazione nella pianificazione di strategie di cura e rigenerazione funzionale del tessuto.

Nel capitolo 3, viene descritta l'analisi comparativa delle unità osteocondrali di diverse specie di mammiferi terrestri e marini, che comprende animali coprenti una gamma ampia di pesi corporei. L'analisi in questione è caratterizzata dallo studio dell'organizzazione microstrutturale e delle caratteristiche biomeccaniche delle diverse unità osteocondrali; questi elementi in particolare vengono scelti per il loro ruolo fondamentale nel funzionamento fisiologico delle articolazioni.

I mammiferi marini rappresentano, in particolare, un gruppo di animali con caratteristiche uniche, essendo animali emersi dall'acqua per compiere una parte del loro processo evolutivo sulla terra e poi tornare nell'ambiente aquatico da cui provengono originariamente tutte le specie. È ipotizzabile che gli effetti evolutivi della locomozione terrestre in questi animali siano scemati dopo il ritorno all'ambiente aquatico e che quindi le differenze microstrutturali osservabili in questi animali siano il risultato dei requisiti diversi per la locomozione richiesti dall'ambiente aquatico.

In questo studio, si osserva che i mammiferi marini mostrano una cartilagine con fibre di collagene essenzialmente non allineate, quindi prive dell'organizzazione ad arco delle specie terrestri, caratterizzata da una gerarchia definita dalla superficie agli strati profondi. Anche l'interfaccia osteocondrale risulta radicalmente diversa nei mammiferi marini, dove manca lo strato della cartilagine calcificata ed è possibile riscontrare una placca subcondrale sottile ed altamente porosa.

Dal punto di vista biomeccanico, nei mammiferi terrestri il modulo elastico di picco risulta significativamente più alto, e in fase di equilibrio la cartilagine risulta più rigida. Le differenze descritte in questo capitolo costituiscono una prova da parte della Natura stessa della grande influenza che ha la distribuzione della massa sulla struttura di osso e cartilagine, e sottolinea l'importanza di un'adeguata compensazione delle esigenze biomeccaniche 
richieste dalle articolazioni.

\section{Parte II}

Le terapie cellulari per la cura della cartilagine finora sono state basate sull'uso di veicoli per il trasporto di cellule rapidamente degradabili come la colla di fibrina. Tuttavia, altri materiali appartenenti alla famiglia degli idrogeli sono stati a lungo oggetto di ricerca come potenziali biomateriali rigenerativi, grazie alla possibilitá di ottimizzare le loro proprietá meccaniche e di trasporto di nutrienti e alle possibilità di funzionalizzazione. Il capitolo 4 affronta la traslazione al modello preclinico equino della gelatina metacrilata (GelMA), come possibile candidato per una terapia rigenerativa della cartilagine, in quanto biomateriale precedentemente utilizzato con successo nel supporto della condrogenesi in vitro. Viene sviluppata una tecnica chirurgica per l'impianto in situ nel ginocchio equino, e successivamente viene eseguito un impianto a breve termine in un pony di razza Shetland, al fine di valutare la fissazione dell'impianto e la fattibilità della tecnica sviluppata. Sia il lavoro svolto ex vivo che lo studio pilota a breve termine dimostrano il successo dell'impianto del GelMA (rinforzato con microfibre di policaprolattone e non), oltre alla persistenza dell'impianto nel difetto. In base a questo successo, viene eseguito uno studio a lungo termine della durata di 12 mesi in entrambe le articolazioni del ginocchio di otto pony di razza Shetland, nelle cui articolazioni viene impiantata una miscela di condroni allogenici e MSCs incapsulati in idrogeli (rinforzati) a base di GelMA e colla di fibrina. Nonostante i risultati incoraggianti dello studio a breve termine, nel corso del monitoraggio dello studio a lungo termine con la tomografia intra-articolare a coerenza ottica si nota che, 2 mesi dopo l'impianto, i rinforzi non sono più presenti. La valutazione istologica eseguita a 12 mesi conferma questa osservazione e dimostra la prevalenza del tessuto fibroso all'interno di tutti i difetti senza differenze significative tra i diversi gruppi sperimentali. Questo quadro è fortemente suggestivo di una degradazione precoce dell'idrogelo e di una sua fissazione inadeguata, che hanno portato all'interno dei difetti ad un processo di riparazione naturale risultante nella formazione di tessuto fibroso (quindi di qualitá inferiore rispetto alla cartilagine nativa).

Il capitolo 5 dunque si incentra sull'ottimizzazione della fissazione degli impianti condrali. Qui vengono valutate colle di fibrina autologa e 
commerciale, ed entrambe dimostrano svantaggi importanti. La colla di fibrina disponibile commercialmente viene sintetizzata per uso negli esseri umani, e se usata nel cavallo, sembra provocare delle reazioni immunologiche avverse di vario grado. L'uso di una colla di fibrina di derivazione autologa non risolve completamente questo problema: la sintesi è complessa e di standardizzazione difficile, richiedendo quindi l'utilizzo addizionale di componenti allogenici, per ottenere un prodotto finale in quantità ridotte e parzialmente immunogenico. Viene quindi descritto lo sviluppo di una soluzione alternativa per la fissazione condrale, consistente in un ancoraggio osseo in policaprolattone (PCL) a cui possono essere aggiunti diversi biomateriali per costituire lo strato condrale, fornendo nel contempo la possibilità di impianto chirurgico di fissazione durevole con un semplice press-fit.

Nei capitoli 6 e 7, l'ancoraggio osteocondrale sviluppato nel capitolo 5 viene usato per indagare gli effetti di una distribuzione cellulare zonale nello strato condrale di uno scaffold impiantato in un difetto equino osteocondrale come modello di rigenerazione cartilaginea. In questo studio, lo scaffold stampato tridimensionalmente in PCL viene provvisto, nella regione condrale, di uno strato di fibre di PCL, atte a rinforzo meccanico, in seguito coperto da due strati di un idrogelo come matrice per incapsulare e trapiantare condrociti. Nel capitolo 6 , viene descritto lo studio in vivo di questa struttura in cui l'idrogelo condrale è formato da acido ialuronico derivatizzato con gruppi tiolo capace di stabilire crosslink tiolo-ene con un polimero sintetico, un poli(glicidolo) (HASH / P(AGE-co-G). Nel capitolo 7, invece, viene utilizzato un idrogelo a base di eparina e glicole polietilenico a quattro bracci funzionalizzato alle estremità (StarPEG/eparina). Lo strato superiore dell'idrogelo contiene cellule progenitrici della cartilagine articolare (ACPC), ottenute dallo strato superficiale del tessuto cartilagineo nativo, mentre lo strato inferiore dell'idrogelo incapsula cellule staminali mesenchimali (MSC). La porzione condrale dei costrutti di controllo invece contiene MSC in tutti gli strati del gel. Sei mesi dopo l'impianto ortotopico in vivo, la tomografia microcomputerizzata rivela una produzione significativa di tessuto osseo nell'ancoraggio di PCL, ed una limitata produzione di tessuto simil-cartilagineo in entrambi i costrutti zonali e non. E' possibile ipotizzare diverse ragioni per questa rigenerazione tissutale limitata, comprese una perdita di cellule impiantate o una degradazione precoce dell'idrogelo. La sostituzione del 
biomateriale con lo starPEG/eparina descritto nel capitolo 7, analizza gli effetti dell'uso di un materiale a degradazione più lenta, confermato dalla presenza dell'idrogel nei difetti sei mesi dopo l'impianto. Tuttavia, questa strategia dimostra una limitata riparazione del tessuto, con risultati comparabili a quelli riportati nel capitolo 6 . 



\section{RÉSUMÉ EN FRANÇAIS}

Cette thèse se concentre sur le développement de stratégies translationnelles pour la réparation (ostéo) chondrale des défauts articulaires à travers le perfectionnement du modèle équin et l'étude morphologique et structurelle des tissus osteo-cartilagineux.

Examiner la biologie fondamentale de l'unité ostéo-cartilagineux chez plus de 40 espèces de mammifères, permet de mieux comprendre la façon dont les différents composants du bloc supportent la charge. L'identification et la compréhension de ces mécanismes sont fondamentales pour la conception d'une stratégie de réparation ostéochondrale efficace.

La translation vers le modèle préclinique équin de stratégies de régénération prometteuses in vitro pour la réparation de défauts est freiné par le manque de stratégies de fixation des implants efficaces et fiables. Cette thèse aborde cette question, perfectionnant ainsi le modèle, jusqu'à passer d'une approche chondrale à une stratégie plus complexe impliquant un échafaudage composite pour la réparation de grands défauts ostéo-cartilagineux.

\section{1ère Partie}

Le cartilage articulaire est un tissu à haut degré de spécialisation qui, au sens fonctionnel, ne peut être apprécié que dans le contexte plus large de l'unité ostéochondrale. Le chapitre $\mathbf{2}$ analyse les mécanismes d'adaptation des dimensions des éléments structurels de l'unité ostéochondrale présents dans une grande variété d'espèces de tailles différentes. Une analyse comparative, effectuée sur le cartilage et l'os de 37 espèces de mammifères, démontre que, bien que l'épaisseur totale du cartilage s'adapte à la masse corporelle de manière allométrique négative, ce n'est pas le cas de chaque différente couche de cartilage. Ce sont principalement les couches de tissu cartilagineux de la zone profonde qui s'adaptent à des masses croissantes, alors que les couches superficielles deviennent relativement plus minces. L'épaisseur de la plaque osseuse sous-chondrale et la fraction osseuse ne montrent aucune corrélation avec la masse corporelle. Au contraire, l'os trabéculaire présente des travées relativement plus épaisses chez les animaux plus gros $(r=0.75, p<0.001)$. Ce résultat était prévisible puisque cette structure supporte la plupart des charges et joue un rôle dans l'atténuation des forces. II ressort de cette étude que la 
structure du tissu ostéo-cartilagineux est remarquablement préservée chez les différentes espèces de mammifères et que c'est principalement l'os trabéculaire qui s'adapte à la masse corporelle croissante. Par ailleurs, la constance et les caractéristiques immuables du cartilage articulaire doivent être pris en considération lors de la conception de stratégies de régénération et de réparation fonctionnelle.

Le chapitre 3, présente une comparaison des unités ostéochondrales de plusieurs espèces de mammifères marins et terrestres couvrant un large éventail de poids corporels. À cet effet, l'organisation microstructurale et les caractéristiques biomécaniques de l'unité ostéochondrale sont examinées en tant qu'aspects essentiels au bon fonctionnement des articulations. Les mammifères aquatiques représentent un groupe unique d'animaux, ayant accompli part de leur évolution sur la terre avant de retourner plus tard à l'environnement aquatique dans lequel toutes les espèces ont évolué à l'origine. On peut donc s'attendre à ce que leurs adaptations originales à la locomotion terrestre aient diminué après le retour au milieu marin et ainsi supposer que les différences microstructurales observées chez ces espèces sont probablement dues aux différentes exigences de la locomotion dans les deux différents environnements.

Cette analyse nous permet de constater que le cartilage des mammifères aquatiques présente des fibres de collagène essentiellement non alignées, sans l'organisation en arcade et dépendante de la profondeur, caractéristique des espèces terrestres. L'interface ostéochondrale se montre aussi très différente chez les mammifères aquatiques qui ne présentent pas de couche de cartilage calcifié et qui ont une fine plaque osseuse sous-chondrale très poreuse. Du point de vue biomécanique, chez les mammifères terrestres le module d'élasticité maximal est significativement plus élevé et, à l'équilibre, le cartilage est plus rigide. Les différences décrites dans cette étude sont la preuve fournie par la nature elle-même de l'énorme influence de la charge sur la structure du cartilage et de l'os, et soulignent l'importance de l'adaptation aux exigences biomécaniques auxquelles l'articulation est soumise.

\section{2ème Partie}

Jusqu'à présent, les thérapies cellulaires pour la réparation du cartilage reposaient principalement sur des supports cellulaires à dégradation rapide 
tels que la colle de fibrine. Cependant, les hydrogels ont aussi été étudiés comme biomatériaux régénératifs potentiels, pour leurs propriétés réglables et leur possibilité de fonctionnalisation. Le chapitre 4 utilise le GelMA (méthacryloyle de gélatine), un hydrogel dont la capacité à soutenir la chondrogenèse avait déjà été démontré in vitro, dans une stratégie régénérative pour la réparation du cartilage dans le modèle animal équin préclinique. On y développe une technique chirurgicale pour l'implantation in situ dans le grasset équin, avant d'effectuer une implantation à court terme chez un poney Shetland afin d'évaluer la faisabilité de la chirurgie et la fixation de I'implant. Les travaux préliminaires ex vivo et l'étude pilote à court terme montrent une implantation réussie de GelMA renforcé et non renforcé (avec des fibres) et la persistance de l'implant dans le défaut. Par conséquent, une étude à long terme de 12 mois est réalisée, durant laquelle un mélange de chondres allogéniques et de cellules souches mésenchymateuses (CSM) englobé dans des hydrogels GelMA (renforcés) et dans de la colle de fibrine est implanté dans les deux articulations fémoro-tibiales de huit poneys Shetland. Cependant, malgré les résultats encourageants à court terme, l'investigation par tomographie intra articulaire à cohérence optique dans l'étude à long terme, indique que 2 mois après l'implantation, les renforts ne sont plus présents. L'évaluation histologique à 12 mois confirme cette observation et révèle la présence de tissu fibreux réparateur comblant tous les défauts, sans aucune différence significative entre les groupes de traitement. Ceci suggère fortement une dégradation précoce de l'hydrogel et une fixation insuffisante, conduisant à un processus de réparation naturel et entraînant la formation de tissu cicatriciel (de moindre qualité).

Le chapitre 5 se focalise sur l'optimisation de la fixation des implants chondraux. Les colles de fibrine commerciales et de dérivation autologue sont évaluées, cependant, les deux approches présentent des inconvénients significatifs. La colle de fibrine commerciale est synthétisée avec des composants humains et, lorsqu'elle est implantée chez le cheval, elle semble provoquer des réactions immunologiques importantes. L'utilisation de colle de fibrine autologue ne paraît pas résoudre complètement ce problème: sa préparation est complexe et difficile à standardiser, et certains composants non équins sont encore nécessaires. En outre le rendement en produit final est peu abondant, tout en provoquant encore une réponse immunologique 
partiale. Le développement d'un ancrage osseux à base de polycaprolactone $(\mathrm{PCL})$ fournit donc une alternative appropriée pour la fixation chondrale, avec la possibilité d'utiliser différents biomatériaux comme couche chondrale, tandis que la partie osseuse offre la possibilité d'une implantation chirurgicale press-fit facile, tout en gardant une fixation durable.

Dans les chapitres 6 et 7, l'ancrage ostéochondrale développé au chapitre 5 est utilisé pour étudier l'effet d'une distribution cellulaire zonale dans la couche chondrale de l'implant dans un modèle de défaut ostéochondral équin pour la réparation du cartilage. Ici, l'ancre osseuse en $\mathrm{PCL}$ imprimée en 3D avec des fibres de renforcement chondral, est recouverte de deux couches d'un hydrogel hybride de acide hyaluronique dérivé avec des groupes thiol capables d'établir des réticulations thiol-ène avec un polymère synthétique, un poly (glycidol) (HA-SH/P(AGE-Co-G)) au chapitre 6, alors qu'au chapitre 7, on utilise un hydrogel à base d'héparine et de polyéthylène glycol à quatre bras fonctionnalisés aux extrémités (StarPEG/héparine). La couche supérieure d'hydrogel contient des cellules progénitrices du cartilage articulaire, dérivées de la couche superficielle de tissu cartilagineux natif, tandis que la couche inférieure contient des cellules souches mésenchymateuses (CSM). La partie cartilagineuse des échafaudages de contrôle est remplie de manière homogène de MSC. Après six mois d'implantation orthotopique in vivo, la tomographie micro-informatisée révèle une production de tissu osseux significative dans l'ancrage osseux en PCL, cependant, la production de tissu de type cartilagineux dans les échafaudages zonales et non zonales est limitée. Cette régénération tissulaire limitée peut s'expliquer par plusieurs raisons, parmi lesquelles la perte précoce des cellules implantées ou la dégradation précoce de I'hydrogel. La substitution de I'hydrogel par StarPEG / héparine comme décrit au chapitre 7 , vise à étudier l'effet d'un taux de dégradation plus lent, confirmé par la persistance du biomatériau dans les défauts six mois après l'implantation. Cependant, cela entraîne également une réparation tissulaire limitée, comparable au résultat rapporté au chapitre 6 . 


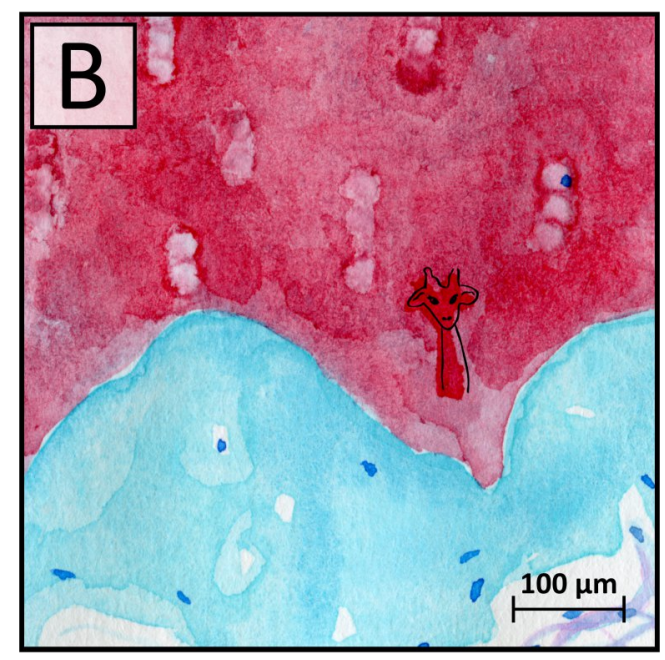


Acknowledgements List of publications

Professional biography 


\section{ACKNOWLEDGEMENTS}

This thesis was driven by passion, effort and wavering determination. Working in a small animal clinic, I often found myself thinking that therapies could be improved, and decided I wanted to make a small contribution. I chose this project because I appreciated the opportunity it gave me to work between animal and human medicine, at the intersection where both can potentially profit from the research conducted. I also hoped it would make me like human doctors better, although I don't know if I have been successful in this aspect. Most of this thesis would not have been possible without some help, so to my collaborators, supervisors and whole support system goes my gratefulness. I will try to be brief (for once): you're all in my heart or in my black book of vengeance. If you're worried you may be in the second group you are today officially forgiven, let's part as friends (of the kind that get in touch for congratulations on important occasions).

I would like, to thank my great promotors, René and Jos, for these years. Thank you for the many scientific (and philosophical) discussions, and for the precious input on manuscripts, projects and ethical issues. You have taught me many lessons. You also gave me all the space needed to find myself as a scientist, and even if I was sometimes critical (sorry, not very positive), I think we are all proud of the work accomplished. René, I also hope one day to become a scientific writer as good as you. Jos, from a small lab on the first/ second floor of the UMC to the spacious and beautiful RMCU grounds, what a journey! Thank you for HydroZONES and for the amazing network it brought. Riccardo, your unwavering dedication and attention to science as well as to your students has been a source of inspiration.

To the many scientists of HydroZONEs, too many to list here: you showed me the potential of scientific collaboration, the good, the great, and sometimes the not-so great, but it has been so exciting sharing this project with you, I am glad to have met you all and learned so much.

Thanks to the always surprising and fruitful collaborators from both the Finnish group headed by unstoppable Prof. Töyräs, and the Costa Rican group headed by the dear friend Prof. Rafael Vindas Bolaños: my experiences abroad 
were so very different, yet in both cases wonderful opportunities for working together, learning and discovering your beautiful countries.

A note of special mention to my Veterinary Faculty collaborators: I am truly grateful for your dedication to our work and to the wellbeing of the horses and ponies. I could not have hoped for a more prepared team of surgeons, anesthesiologists, researchers, technicians and care-takers. Prof. Brommer, Harold, thank you for the impeccable coordination and collaboration.

A thought goes to my many colleagues from RMCU, UU and UMC: you all made the journey much more enjoyable, full of interesting discussions during coffee breaks, lab events, visits to the pannenkoekehuis, cookies and cakes in way too many occasions! To those of you who have gotten to know me more, your support and kindness even when it wasn't your job have meant so much,I hope you know who you are.

Finally, I would like to thank my chosen family, so far away, yet always managing to be close somehow. Dilek, Cécile and Silvia, words are not good enough, so I will just say that I am so lucky to have found you, and miss you terribly.

Husband, I could not have asked for a better partner in crime, I will never get tired of professing all my love for you... let's talk in private if you know what I mean.

To those left in my family still speaking to me or alive, you're okay too.

Paranymphs (Fred and Ale), it's time to celebrate! 


\section{LIST OF PUBLICATIONS}

I.A.D. Mancini, R. Levato, M. Castilho, M. Chen, M.H.P. van Rijen, M. Kik, J. Malda, P.R. van Weeren. Nature's design of the osteochondral unit: microstructural differences between terrestrial and aquatic mammals. Submitted

I.A.D. Mancini, J. Malda, R. Levato, H. Brommer, F. Schrön, C. Warner, P.R. van Weeren. Addendum to "A composite hydrogel-3D printed thermoplast osteochondral anchor as example for a zonal approach to cartilage repair: in vivo performance in a long-term equine model". Submitted

I.A.D. Mancini, S. Schmidt, H. Brommer, B. Pouran, S. Schäfer, J. Tessmar, A. Mensinga, M.H.P. van Rijen, J. Groll, T. Blunk, R. Levato, J. Malda, P.R. van Weeren. A composite hydrogel-3D printed thermoplast osteochondral anchor as an example for a zonal approach to cartilage repair: in vivo performance in a long-term equine model. Biofabrication, 2020.

A.W. Kajabi, V. Casula, J.K. Sarin, J.H. Ketola, O. Nykänen, N.C.R Te Moller, I.A.D. Mancini, J. Visser, H. Brommer, P.R. van Weeren, J. Malda, J. Töyräs, M.T. Nieminen, M.J. Nissi. Evaluation of articular cartilage with quantitative MRI in an equine model of post-traumatic osteoarthritis. Journal of Orthopaedic Research, 2020.

O. Nykänen, J.K. Sarin, J.H. Ketola, H. Leskinen, N.C.R. Te Moller, V. Tiitu, I.A.D. Mancini, J. Visser, H. Brommer, P.R. van Weeren, J. Malda, J. Töyräs, M.J. Nissi. T2* and quantitative susceptibility mapping in an equine model of posttraumatic osteoarthritis: assessment of mechanical and structural properties of articular cartilage. Osteoarthritis and cartilage, 2019. 27;10:1481-1490.

I.A.D. Mancini, L. Rieppo, B. Pouran, I.O. Afara, F.M.S. Braganca, M.H.P. van Rijen, M. Kik, H. Weinans, J. Toyras, P.R. van Weeren, J. Malda. Effects of body mass on microstructural features of the osteochondral unit: A comparative analysis of 37 mammalian species. Bone, 2019. 127:664-673.

J.K. Sarin, O. Nykänen, V. Tiitu, I.A.D. Mancini, H. Brommer, J. Visser, J. Malda, 
P.R. van Weeren, I.O. Afara, J. Töyräs. Arthroscopic determination of cartilage proteoglycan content and collagen network structure with Near-Infrared Spectroscopy. Annals of Biomedical Engineering, 2019. 47;8:1815-1826.

J.K. Sarin, N.C.R. Te Moller, I.A.D. Mancini, H. Brommer, J. Visser, J. Malda, P.R. van Weeren, I.O. Afara, J. Töyräs. Arthroscopic near infrared spectroscopy enables simultaneous quantitative evaluation of articular cartilage and subchondral bone in vivo. Scientific Reports, 2018. 8;1-13409.

I.A.D. Mancini, R. Vindas Bolaños, H. Brommer, M. Castilho, A. Ribeiro, J.P.A.M. van Loon, A. Mensinga, M.H.P. van Rijen, J. Malda, R. van Weeren. Fixation of hydrogel constructs for cartilage repair in the equine model: a challenging issue. Tissue engineering Part C: methods, 2017. 23;11:804-814. 


\section{PROFESSIONAL BIOGRAPHY}

Irina Anna Diletta Mancini was born in Rome on the 15th of March 1985. She spent her first years of life in the city of Lecce, in South Italy, but her parents' professional endeavours brought her to move to different continents and Italian cities from the age of 9 . At the same age she rescued a bat and decided she wanted to study veterinary medicine. During her studies in veterinary medicine, she became interested in surgery, anaesthesiology and exotic animals' medicine. She spent a period of her studies as intern at the Parco Natura Viva in Bussolengo, Italy, and over a year and a half as anaesthesiology intern in the Small Animals Surgery Department of Parma University, where she initiated a clinical trial on the use of amantadine for chronic pain management in osteoarthritic dogs, which became the subject of her thesis, concluded in April 2012.

In the university years, she also took time off to help run her family business, and dedicated time to volunteer work in an wild animal rescue centre, and later in the Italian Red Cross.

In 2012 an earthquake also hit her partner's hometown hindering the local economy, so together they decided to expatriate and moved to Nottinghamshire where Irina completed a 13 month post-graduate internship at the Pride Veterinary Centre. During this time she had the opportunity to learn and practice animal care of the best quality and observed that this was still insufficient in some cases. This reflection lead her to be interested in new strategies such as regenerative medicine, so she took the opportunity to study this field closely and moved to Netherlands in 2013 to start her PhD under the Regenerative Medicine Program of Utrecht University, under the supervision of Prof. P.R. van Weeren and Prof. J. Malda. The results of her research are presented in this thesis.

In the later portion of her PhD Irina identified communication of science as an important issue for her, and worked part-time as communication officer of the Regenerative Medicine Centre Utrecht (RMCU). In her continuous quest to contribute to society and passionately promote her ideas, she founded in 2019 an independent science visual communication company, She Illustrates Science. The company's scope is to help scientists explain their science, findings or ideas for projects through illustrations, videos, infographics, and 
soon also workshops on effective communication.

Irina enjoys the most hiking in her dear Dolomite mountains, swimming in the beautiful portion of Mediterranean Sea near Lecce, and being creative in many different ways.

She currently lives in Utrecht with her husband, her 16 year old cat, a very spoiled black dog, 5 fishes and a Japanese shrimp called NotYetDimSum. 
...Buccina però potevi aspettare un po'! 



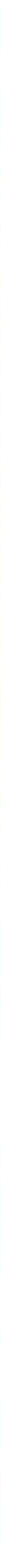

